Copyright

by

Tongcang Li

2011 
The Dissertation Committee for Tongcang Li

certifies that this is the approved version of the following dissertation:

\section{Fundamental tests of physics with optically trapped microspheres}

Committee:

Mark G. Raizen, Supervisor

Daniel J. Heinzen

Alan W. Reid

Greg O. Sitz

Vernita Gordon 


\title{
Fundamental tests of physics with optically trapped microspheres
}

\author{
by
}

Tongcang Li, B.S.

\author{
DISSERTATION \\ Presented to the Faculty of the Graduate School of \\ The University of Texas at Austin \\ in Partial Fulfillment \\ of the Requirements \\ for the Degree of \\ DOCTOR OF PHILOSOPHY
}

THE UNIVERSITY OF TEXAS AT AUSTIN

May 2011 
Dedicated to my parents, Daogan Li and Zhangman Huang, my wife, Ruyi Li, and my sister, Xiaoping Li, for their endless love and support. 


\section{Acknowledgments}

First, I would like to thank my supervisor, Dr. Mark Raizen, for providing me the opportunity to work in an outstanding group. Mark is a great person to work with and is full of ideas. I am impressed by his vision and confidence. Mark often proposes ambitious ideas that may appear suspicious to most people at first. During my time in his group, I had the privilege to witness these ideas ultimately realized in ground-breaking experiments. I could not have asked for a better advisor to learn how to do creative research.

I joined the lab in Nov. 2004 to work on the sodium Bose-Einstein condensate (BEC) experiment with Kevin Henderson and Hrishikesh Kelkar. Kevin was very helpful and excellent in teaching. I learned a lot of experimental skills from him, including driving a car. Hrishi was extremely patient and hard working. He aligned a lot of difficult parts of the optics, some of which took several weeks of continuous work. David Medellin joined this experiment in 2006. David is fantastic with mathematics and physical concepts, which is very helpful to our work. He is always trying his best to make his work perfect. I also enjoyed the dinners with Hrishi and David when we were working together. Kirsten Viering joined this experiment as a Würzburg exchange student and later returned back after working on a Rubidium experiment. She is a very good experimentalist and works very efficiently. 
I started the new experiment on optical trapping and cooling of microspheres with the help of David Medellin. A particular thanks goes to Simon Kheifets. He joined this experiment just after we were able to trap microspheres in vacuum and has participated in almost all aspects of this experiment since then. He worked very hard and helped me to make this experiment fruitful. I also enjoyed the discussions with him on our new ideas almost every day. I fully expect that he will push the experiment to an unprecedented level. I would also want to thank Ivo Popov, Ioannis K. Charidakos, and Isaac Chavez for their contributions to this experiment.

I would like to thank other students in the lab who have provided much help to me. They are Braulio Gutierrez-Medina, Todd Meyrath, Chih-Sung Chuu, Gabriel Price, Travis Bannerman, Ed Narevicius, Adam Libson, Rob Clark, Tom Mazur, Melissa Jenkins, and Jianyong Mo. I would also like to thank Charlotte Sanders, Francisco Camargo, Daniel Ellsworth, Julia Majors, Christoph Schaeff, Christian Parthey, Max F. Riedel, Artem M. Dudarev, Chuanwei Zhang, and Shoupu Wan for making the environment of our lab friendly and enjoyable.

I would like to thank Jack Clifford, Allan Schroeder and all other members of the machine shop, and Ed Baez and Larry Sandefur in the cryo lab for their excellent work. I would like to thank Olga Vera, Elena Simmons, Marybeth Casias, and other members of CNLD for their administrative help.

I also appreciate the friendships from Shaoping Lu, Hui Chen, Ning Kong, Huai Huang, Jiaxin Han, Tianyi Yang, Zhangqi Yin, Ming Lei, Kin 
Chung Fong, and more.

Finally, I would definitely like to thank my wife, Ruyi Li. She is very supportive and provides me a sweet home. I also want to thank my parents and my sister for their endless love and encouragement.

Tongcang Li

April 28, 2011

Austin, TX 


\section{Fundamental tests of physics with optically trapped microspheres}

Publication No.

Tongcang Li, Ph.D.

The University of Texas at Austin, 2011

Supervisor: Mark G. Raizen

This dissertation details our experiments on studying the Brownian motion of an optically trapped microsphere with ultrahigh resolution, and cooling of its motion towards the quantum ground state.

We have trapped glass microspheres in water, air and vacuum with optical tweezers. We developed a detection system that can monitor the position

of a trapped microsphere with Ångstrom spatial resolution and microsecond temporal resolution. We studied the Brownian motion of a trapped microsphere in air over a wide range of pressures. We measured the instantaneous velocity of a Brownian particle. Our results provide direct verification of the Maxwell-Boltzmann velocity distribution and the energy equipartition theorem for a Brownian particle. For short time scales, the ballistic regime of Brownian motion is observed, in contrast to the usual diffusive regime. We are currently developing a new detection system to measure the instantaneous velocity of a Brownian particle in water.

viii 
In vacuum, we have used active feedback to cool the three center-ofmass vibration modes of a trapped microsphere from room temperature to millikelvin temperatures with a minimum mode temperature of $1.5 \mathrm{mK}$, which corresponds to the reduction of the root mean square (rms) amplitude of the microsphere from $6.7 \mathrm{~nm}$ to $15 \mathrm{pm}$ for that mode. The mean thermal occupation number of that mode is reduced from about $6.8 \times 10^{8}$ at $297 \mathrm{~K}$ to about 3400 at $1.5 \mathrm{mK}$. 


\section{Table of Contents}

Acknowledgments $\quad$ v

Abstract viii

List of Tables $\quad$ xiii

List of Figures $\quad$ xiv

Chapter 1. Introduction 1

1.1 Macroscopic quantum mechanics . . . . . . . . . . . . . . . . 1

1.2 Instantaneous velocity of Brownian motion . . . . . . . . . . 5

1.3 Contents of this dissertation . . . . . . . . . . . . . . . . . 9

Chapter 2. Physical principle of optical tweezers 11

2.1 Ray optics approximation . . . . . . . . . . . . . . . . . . 12

2.2 Rayleigh approximation . . . . . . . . . . . . . . . . . . 14

2.3 Generalized Lorentz-Mie theory . . . . . . . . . . . . . . . . 21

Chapter 3. Optical trapping of glass microspheres in air and vacuum 27

3.1 Launching microspheres . . . . . . . . . . . . . . . . . 28

3.2 Trapping microspheres . . . . . . . . . . . . . . 38

3.2.1 Optical levitation trap . . . . . . . . . . . . . . 39

3.2.2 Counter-propagating dual-beam optical trap . . . . . . 42

3.3 Vacuum system . . . . . . . . . . . . . . . 52

Chapter 4. Measuring the instantaneous velocity of a Brownian $\begin{array}{ll}\text { particle in air } & 57\end{array}$

4.1 Theories of Brownian motion . . . . . . . . . . . . . 58

4.1.1 A free particle . . . . . . . . . . . . 58 
4.1.2 A trapped microsphere . . . . . . . . . . . . . . 61

4.1.2.1 Displacement and velocity . . . . . . . . . . . 61

4.1.2.2 Power spectrum analysis . . . . . . . . . . . 63

4.2 A fast detection system . . . . . . . . . . . . 66

4.3 Measured power spectra . . . . . . . . . . . . . . 71

4.4 Measurement of the instantaneous velocity of a Brownian particle in air . . . . . . . . . . . . . . . 76

Chapter 5. Towards measurement of the instantaneous velocity of a Brownian particle in water $\quad 86$

5.1 Motivation . . . . . . . . . . . . . . . . 86

5.2 Hydrodynamic theories of Brownian motion . . . . . . . . 87

5.2 .1 A free particle in water . . . . . . . . . . 87

5.2.2 An optically trapped microsphere in water . . . . . . 89

5.3 Requirements for measuring the instantaneous velocity . . . . 97

5.4 A simple optical tweezer in water . . . . . . . . . . . . 102

5.5 Interferometer-enhanced optical tweezers . . . . . . . . . . 111

\section{Chapter 6. Millikelvin cooling of an optically trapped micro-} $\begin{array}{lr}\text { sphere in vacuum } & 117\end{array}$

6.1 Background . . . . . . . . . . . . . . . . . . . . . 117

6.2 Principle of feedback cooling . . . . . . . . . . . . . . 120

6.2 .1 Feedback cooling . . . . . . . . . . . . . . . . . . . 121

6.2 .2 Feedback amplification . . . . . . . . . . . . . . . . 122

6.2 .3 Heating due to light scattering . . . . . . . . . . . . . 123

6.2.4 Damping due to the residual gas in vacuum . . . . . . 125

6.3 A 3D split detection system _ . . . . . . . . . . . . 128

6.4 1D optical feedback cooling . . . . . . . . . . . . . . . . . . 133

6.5 Electrostatic forces . . . . . . . . . . . . . . . . . 141

6.6 Millikelvin cooling with 3D optical feedback . . . . . . . 147

6.6 .1 Experimental setup . . . . . . . . . . . . . . . 147

6.6.2 Results of 3D optical feedback cooling . . . . . . . . . 152

6.7 Loss of microspheres in vacuum . . . . . . . . . 156 
Chapter 7. Towards quantum ground-state cooling 163

7.1 Principle of cavity cooling . . . . . . . . . . . . . 163

7.2 3D ground-state cooling with a single cavity . . . . . . 167

7.3 Heating effects of laser noise . . . . . . . . . . . . . 171

7.4 Applications of cooled microspheres in vacuum . . . . . . . . 173

7.4 .1 Measuring weak forces . . . . . . . . . . . . . . . 173

7.4.2 Measuring the impact of single molecules . . . . . . . 175

7.4.3 Searching for gravity-induced quantum-state reduction . 177

Appendices

179

Appendix A. Physical properties of some common materials 180

$\begin{array}{ll}\text { Bibliography } & 181\end{array}$

$\begin{array}{ll}\text { Vita } & 200\end{array}$ 


\section{List of Tables}

3.1 Required ultrasonic powers for launching . . . . . . . . . . . 35

5.1 Characteristic time scales of a particle in water . . . . . . . 91

5.2 Required resolution for measuring the instantaneous velocity . 98

6.1 Lifetime of a trapped microsphere in vacuum . . . . . . . . . . 157

A.1 Physical properties of some common materials . . . . . . . . 180

xiii 


\section{List of Figures}

1.1 A 2D trajectory of a Brownian particle .......... 6

2.1 Qualitative view of optical trapping . . . . . . . . . . . 13

2.2 Schematic of a nanosphere in a laser beam . . . . . . . . . . . 15

2.3 Potentials and forces on a nanosphere in air (waist $=1.5 \mu \mathrm{m}$ ) 19

2.4 Potentials and forces on a nanosphere in air (waist $=0.5 \mu \mathrm{m}$ ) 20

2.5 Optical forces on a microsphere in water . . . . . . . . . . . 23

2.6 Optical forces on microspheres in air . . . . . . . . . . . . . . 24

2.7 Optical forces on microspheres in air v.s. size and NA . . . . . 25

2.8 Minimum forces on a microsphere in air v.s. size . . . . . . . . 26

3.1 Photos of a home-built ultrasonic transducer . . . . . . . . . . 30

3.2 Schematic of a simple ultrasonic transducer . . . . . . . . . . 31

3.3 Glass microspheres after ultrasonic vibration . . . . . . . . . . 32

3.4 Impedance of a piezoelectric ceramic ring . . . . . . . . . . . . 34

3.5 Electronic circuit of the pulsed generator . . . . . . . . . . 36

3.6 Photo of a high power pulsed generator . . . . . . . . . . . . 37

3.7 An optical levitation trap . . . . . . . . . . . . . . . . . . 40

3.8 A levitated microsphere in air . . . . . . . . . . . . . . . . . 41

3.9 Schematic of a dual-beam optical trap . . . . . . . . . . . . . 43

3.10 A misaligned dual-beam optical trap . . . . . . . . . . . . . . 44

3.11 First generation dual-beam trap in vacuum . . . . . . . . . . . 46

3.12 Setup inside the vacuum chamber . . . . . . . . . . . . . . . . 47

3.13 Setup for aligning laser beams . . . . . . . . . . . . . . . . . 48

3.14 Measured beam profiles . . . . . . . . . . . . . . . . 49

3.15 A $4.7-\mu \mathrm{m}$ bead trapped inside a vacuum chamber . . . . . . . 50

3.16 Images of trapped microspheres . . . . . . . . . . . . . . . . 51

3.17 Vacuum system . . . . . . . . . . . . . . . 53

xiv 
4.1 Rms amplitude of a trapped microsphere . . . . . . . . . . . 62

4.2 Calculated spectra in linear scales . . . . . . . . . . . . . . 64

4.3 Calculated spectra in $\log -\log$ scales . . . . . . . . . . . 65

4.4 1D split detector . . . . . . . . . . . . . . . 67

4.5 Optical circuit of a dual-beam trap . . . . . . . . . . . . . . . 69

4.6 Spectrum of a $3.0-\mu \mathrm{m}$ bead trapped at 749 torr . . . . . . . . 72

4.7 Spectrum of a $3.0-\mu \mathrm{m}$ bead trapped at 20.6 torr . . . . . . . . 73

4.8 Spectrum of multiple $3.0-\mu$ m beads trapped at 752 torr . . . . 74

4.9 Spectra of two $4.7-\mu \mathrm{m}$ beads trapped at 9.78 torr . . . . . . . 75

4.10 Measured instantaneous position and velocity of a bead . . . . 78

4.11 Measured mean square displacement of a bead . . . . . . . . . 79

4.12 Measured velocity distribution of a bead . . . . . . . . . . . 81

4.13 Measured velocity correlation of a bead . . . . . . . . . . . . . 82

4.14 Measured $\tau_{p}$ v.s. laser power . . . . . . . . . . . . . . . 84

5.1 MSD of a Brownian particle in water . . . . . . . . . . . . 92

5.2 Spectrum of a Brownian particle in water . . . . . . . . . . . 93

5.3 VACF of a Brownian particle in water . . . . . . . . . . . 95

5.4 VACF of a Brownian particle in water at $20^{\circ} \mathrm{C}$ and $60{ }^{\circ} \mathrm{C}$. . 96

5.5 An optical tweezer in water . . . . . . . . . . . . 103

5.6 A 4.7- $\mu \mathrm{m}$ diameter microsphere trapped in water . . . . . . . 105

5.7 Noise spectra of detectors . . . . . . . . . . . . . . . . . . . . 107

5.8 Spectrum of a $4.7-\mu \mathrm{m}$ bead in water 1 . . . . . . . . . . . 108

5.9 Spectrum of a $4.7-\mu \mathrm{m}$ bead in water 2 . . . . . . . . . . . . 109

5.10 MSD of a 4.7- $\mu$ m bead in water . . . . . . . . . . . . . . . . . 110

5.11 Mach-Zehnder interferometer enhanced optical tweezer . . . . 114

5.12 Wollaston interferometer enhanced optical tweezer . . . . . . . 115

6.1 Oscillation linewidth v.s. air pressure . . . . . . . . . . . 126

6.2 Power spectrum at 1 mtorr . . . . . . . . . . . . . . . . 127

6.3 Schematic of a 3D detection system . . . . . . . . . . . . . 130

6.4 A photo of a 3D detection system . . . . . . . . . . . . . 131

6.5 Scheme of 1D feedback cooling . . . . . . . . . . . . . 134 
6.6 Derivative circuit . . . . . . . . . . . . . . . . . 135

6.7 Results of 1D feedback cooling . . . . . . . . . . . . . 137

6.8 Direction modulation of beam No. 2 . . . . . . . . . 138

6.9 Direction modulation of beam No. 1 . . . . . . . . . . 140

6.10 Setup for air discharge . . . . . . . . . . . . . . . . 141

6.11 Spectra of air discharge . . . . . . . . . . . . . . . . . 142

6.12 Spectrum at 205 torr after air discharge . . . . . . . . . 143

6.13 Charge fluctuation . . . . . . . . . . . . . . . . 145

6.14 Schematic of trapping beams and 3D cooling beams . . . . . 148

6.15 Diagram of the feedback mechanism for the X axis . . . . . 149

6.16 A photo of our 3D feedback cooling system . . . . . . . . 150

6.17 Control the laser power with an AOM . . . . . . . . . 151

6.18 Power spectra of a bead along $\mathrm{X}$ axis . . . . . . . . . . 153

6.19 Power spectra of a bead along $\mathrm{Y}$ axis . . . . . . . . . . 154

6.20 Power spectra of a bead along $\mathrm{Z}$ axis . . . . . . . . . . 155

6.21 Temperature v.s. air pressure . . . . . . . . . . 156

6.22 Internal temperature of a microsphere . . . . . . . . . 160

7.1 1D cavity cooling scheme . . . . . . . . . . . . . . . . 164

7.2 3D cavity cooling scheme . . . . . . . . . . . 168

7.3 Minimum detectable force . . . . . . . . . . . . . . . 174

7.4 Photon number increase after molecular collisions . . . . . . 176 


\section{Chapter 1}

\section{Introduction}

\subsection{Macroscopic quantum mechanics}

It is well known that the dynamics of microscopic particles such as photons, electrons and atoms follow quantum mechanics, while the dynamics of macroscopic objects such as ping-pong balls follow classical mechanics. Why is there such a transition?

A simple explanation for the absence of quantum behavior of macroscopic objects is that the de Broglie wavelength $\lambda$ of an object is inversely proportional to the momentum $p$ of the object, i.e. $\lambda=h / p$, where $h$ is the Planck constant. Macroscopic objects usually have large momenta, thus their de Broglie wavelengths and quantum behaviors are too small to be observed.

Since $p=M v$, where $M$ is the mass of an object and $v$ is the velocity of the object, we can increase the de Broglie wavelength of an object by reducing its velocity. Thus macroscopic objects should behave quantum mechanically if we can reduce their kinetic energies to low enough values. An important characteristic of quantum mechanics is the possibility of superposition of two spatially distinct states. Creation of quantum superpositions of macroscopic objects ("Schrödinger cats") will provide opportunities to study 
untested regimes of quantum mechanics.

One additional motivation to study the macroscopic quantum mechanics is that the apparent conflict between general relativity and quantum mechanics remains one of the unresolved mysteries of the physical world [1,2]. According to recent theories [2-4], this conflict results in gravity-induced quantum state reduction of "Schrödinger cats". It is argued that the perturbing effect of the mass distribution on the space-time structure leads to an essential uncertainty in the energy of a superposed state. So a macroscopic quantum superposition of two different mass distributions is intrinsically unstable.

The finite lifetime of a quantum superposition due to the gravityinduced state reduction is on the order of $\tau=\hbar / \Delta E$, where $\hbar$ is the Planck constant/2 $2 \pi . \Delta E$ is the gravitational self-energy of the difference between the mass distributions of the two superposed states $[2,5,6]$ :

$$
\Delta E=\xi G \iint \frac{\left[\rho(\mathbf{x})-\rho^{\prime}(\mathbf{x})\right] \cdot\left[\rho(\mathbf{y})-\rho^{\prime}(\mathbf{y})\right]}{|\mathbf{x}-\mathbf{y}|} d \mathbf{x} d \mathbf{y}
$$

where $G$ is Newton's gravitational constant, $\rho$ and $\rho^{\prime}$ are the mass densities of two members of the superposed state. $\xi$ is a dimensionless number that represents the strength of the quantum state reduction process, which is expected to be on the order of 1 . The lifetime $\tau$ of a quantum superposition of two separated states of a 1-nm-radius particle is on the order of $10^{13} \mathrm{~s}$, which is too long to be measured in an experiment. It is on the order of $10^{-17} \mathrm{~s}$ for a $1 \mathrm{~mm}$ particle, which is too short to be measured. For a $1 \mu \mathrm{m}$ particle, the lifetime of a spatially separated quantum superposition is on the order of 
$10^{-2} \mathrm{~s}$, which seems realistic to be measured in an experiment.

In order to observe quantum behaviors in a mechanical oscillator, we need to cool the oscillator significantly. The energy of the quantum states of a mechanical oscillator is quantized in units of $\hbar \Omega_{M}$, where $\Omega_{M}$ is the angular frequency of the oscillator. The mean thermal occupation number (phonon number) is $\langle n\rangle=k_{B} T / \hbar \Omega_{M}$, where $k_{B}$ is the Boltzmann constant, and $T$ is the temperature of the oscillator. At room temperature, we have $\langle n\rangle \gg 1$ for almost all mechanical oscillators. We need $\langle n\rangle<1$ for the oscillator to have a high probability to be in the quantum ground state and to have its dynamics be dominated by quantum mechanics.

In recent years, great progress has been made in cooling mechanical oscillators towards their quantum mechanical ground states [7-9]. The thermal occupation number of a $2.7 \mathrm{~kg}$ pendulum mode around $130 \mathrm{~Hz}$ was reduced from about $5 \times 10^{10}$ at room temperature to about 234 at an effective temperature of $1.4 \mu \mathrm{K}$ by feedback cooling [10]. Combining conventional cryogenic cooling and laser cavity cooling, the thermal occupation number of a mechanical deformation mode of a deformed microsphere around $100 \mathrm{MHz}$ was reduced to about 40 [11], the thermal occupation number of the fundamental mechanical mode of a micromirror (the end mirror of a Fabry-Pérot cavity) at $945 \mathrm{kHz}$ was reduced to about 30 [12]. Remarkably, quantum ground-state cooling has been achieved for a dilatational resonant mode of a membrane at $6.2 \mathrm{GHz}$ by conventional cryogenic cooling [13], and for the fundamental mechanical mode of a superconducting aluminium membrane at $10.56 \mathrm{MHz}$ by microwave cavity 
cooling [14].

These results are important steps towards the creation of Schrödinger cats in the laboratory, and the study of their destruction by decoherence. A direct test of the gravity-induced state reduction scenario may therefore be within reach. However, a recent analysis shows that for these mechanical oscillators, quantum superpositions are destroyed by environmental decoherence long before gravitational state reduction takes effect [6]. An alternate candidate may be a Bose-Einstein condensate (BEC) of ultracold atoms in vacuum. It is well isolated from the thermal environment. However, its mass density is too small. Even worse, the atoms in a Bose-Einstein condensate are only weakly interacting. They do not move together. Thus only mass fluctuation, not total mass, contributes to the $\Delta E$. Theoretical calculation shows that a Bose-Einstein condensate formed from $10^{29}$ atoms is required to study the gravity-induced state reduction [6]. This number is too large to be achieved in the foreseeable future.

Chapters 3 and 6 of this dissertation describe our experimental efforts on optical trapping and cooling of glass microspheres in vacuum [15]. An optically trapped microsphere in vacuum provides an ideal candidate for studying the interface between quantum mechanics and general relativity, and for studying objective collapse models of the wavefunction [16]. It is nearly perfectly isolated from the thermal environment, and allows quantum ground state cooling from room temperature [17-19]. The mass density of a glass microsphere is many orders larger than that of a BEC, and atoms contained in the micro- 
sphere move together as a whole. We have been able to trap glass microspheres in vacuum with high oscillation frequencies, and cool their center-of-mass motion from room temperature to a minimum temperature of about $1.5 \mathrm{mK}$.

\subsection{Instantaneous velocity of Brownian motion}

Brownian motion is the apparently random movement of particles suspended in a fluid (liquid or gas). It was discovered by Robert Brown (17731858 ) in 1827 when he used a simple microscope (a double convex lens with a focal length of about $0.8 \mathrm{~mm}$ ) to study the action of particles contained in the grains of pollens [20]. The size of those particles was about $5 \mu \mathrm{m}$. He "observed many of them very evidently in motion". He also observed the same motion with powders of many other materials, such as wood and nickel, suspended in water.

Persistence and randomness are two key characteristics of Brownian motion. The trajectories of a Brownian particle are classic examples of fractals. They are commonly thought to be continuous everywhere but not differentiable anywhere. Since its trajectory is not differentiable, the velocity of a Brownian particle is undefined.

At short time scales, however, the dynamics of a Brownian particle is expected to be dominated by its inertia and its trajectory cannot be selfsimilar. This is termed "ballistic Brownian motion" to be distinguished from the common "diffusive Brownian motion". Fig. 1.1 shows a 2D trajectory of a Brownian particle. The black curve is assumed to be a true trajectory of the 
particle. Red dots are measured positions. In Fig. 1.1A, the sampling rate is too small to measure the velocity of the Brownian particle. The measured trajectory (red curve) is completely different from the true trajectory, and appears chaotic. It is impossible to obtain the velocity of the particle from the measured trajectory in Fig. 1.1A. In Fig. 1.1B, the sampling rate is much larger. Now the measured trajectory is very close to the true trajectory of the particle. If the measured displacement of the particle is $\Delta \vec{x}(t)$ during time $\Delta t$, then the velocity of the particle is approximately $\vec{v}=\Delta \vec{x}(t) / \Delta t$.

In 1900, F. M. Exner made the first quantitative study of Brownian motion by measuring the velocity of the Brownian motion of particles suspended in water $[21,22]$. He found that the measured velocity decreased with increasing particle size and increased with increasing water temperature. However,

(A)

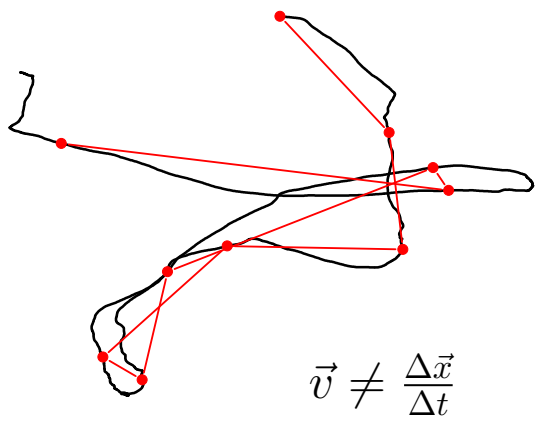

(B)

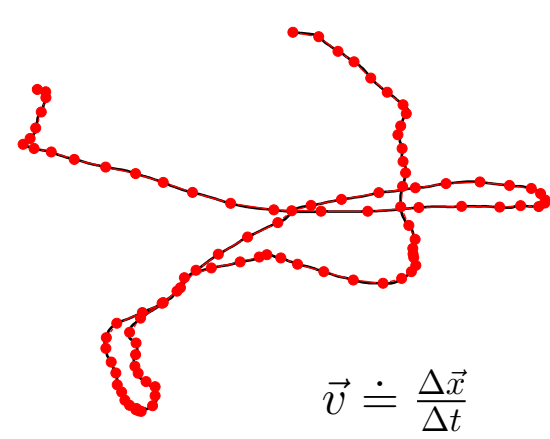

Figure 1.1: A 2D trajectory of a Brownian particle. The black curve is assumed to be a true trajectory of the particle. Red dots are measured positions, and red curves are measured trajectories. The sampling rate of (B) is 10 times of that of $(\mathrm{A})$. 
his measured velocities of Brownian particles were almost 1000-fold smaller than those predicted by the energy equipartition theorem [21]. The reason of this discrepancy was not understood until A. Einstein developed a kinetic theory about Brownian motion in 1905 [23].

In 1907, Einstein published a paper entitled "Theoretical observations on the Brownian motion" in which he considered the instantaneous velocity of a Brownian particle $[24,25]$. Einstein showed that by measuring this quantity, one could prove that "the kinetic energy of the motion of the centre of gravity of a particle is independent of the size and nature of the particle and independent of the nature of its environment". This is one of the basic tenets of statistical mechanics, known as the equipartition theorem. However, Einstein concluded that due to the very rapid randomization of the motion, the instantaneous velocity of a Brownian particle would be impossible to measure in practice $[24,25]$ :

"We must conclude that the velocity and direction of motion of the particle will be already very greatly altered in the extraordinary short time $\theta^{\mathrm{i}}$, and, indeed, in a totally irregular manner. It is therefore impossible - at least for ultramicroscopic particles - to ascertain $\sqrt{\overline{\overline{v^{2}}}}$ by observation."

The resolution required to measure the instantaneous velocity is striking. For a $1 \mu \mathrm{m}$ diameter silica $\left(\mathrm{SiO}_{2}\right)$ sphere in water at room temperature,

\footnotetext{
in Einstein's paper, $\theta$ was defined as the time in which the velocity falls to a tenth of its original value. $\theta=2.30 \tau_{p}$, where $\tau_{p}$ is the momentum relaxation time of the particle. Einstein mistakenly obtained $\theta=330$ ns for a 50 -nm-diameter platinum nanosphere in water. The correct value should be $\theta=6.8 \mathrm{~ns}$ for his example.
} 
the momentum relaxation time is about $0.1 \mu$ s and the root mean square (rms) velocity $v_{r m s}=\sqrt{k_{B} T / M}$ is about $2 \mathrm{~mm} / \mathrm{s}$ in one dimension. To measure the instantaneous velocity with $10 \%$ uncertainty, one would require 2 pm spatial resolution in 10 ns.

Recent experiments on Brownian motion in liquid [26-29] and gaseous environments [30-32] with fast detectors have observed nondiffusive motion of a Brownian particle. However, no previous experiment has been able to observe the instantaneous velocity of a Brownian particle and verify the energy equipartition theorem directly.

Chapter 4 of this dissertation describes our efforts to measure the instantaneous velocity of a Brownian particle suspended in air [33]. Due to the lower viscosity of gas as compared to liquid, the momentum relaxation time $\tau_{p}$ of a particle in air is much larger. This lowers the technical demand for both temporal and spatial resolution. The main difficulty of performing high precision measurements of a Brownian particle in air, however, is that the particle will fall under the influence of gravity. We overcome this problem by using optical tweezers to simultaneously trap and monitor a silica bead in air and vacuum, allowing long duration ultra-high-resolution measurements of its motion.

We have successfully measured the instantaneous velocity of a Brownian particle in air. We used the velocity data to directly verify the MaxwellBoltzmann velocity distribution, and the equipartition theorem for a Brownian particle. The ability to measure instantaneous velocity enables new funda- 
mental tests of statistical mechanics of Brownian particles [34-37]. It is also a necessary step towards cooling of a particle to the motional quantum ground state in vacuum.

We are currently building a new detection system to measure the instantaneous velocity of a Brownian particle in water (chapter 5). In contrast to

air, the water has significant inertia and hydrodynamic memory effects [26, 29], which may cause deviations from the energy equipartition theorem.

\subsection{Contents of this dissertation}

Chapter 2 introduces the basic principle of optical tweezers, and the differences between trapping microspheres in air and in water. Chapter 3 provides the details of launching glass microspheres to air, and trapping microspheres in air and vacuum with a counter-propagating dual-beam optical tweezer. We also describe the vacuum system and the first generation of our detection system. Chapter 4 covers the theory of Brownian motion in air at short time scales, a home-built detection system with ultrahigh resolution, and the results of our measurement of the instantaneous velocity of a Brownian particle in air. Chapter 5 discusses the motivation and challenges of measuring the instantaneous velocity of a Brownian particle in water. It also presents some of our on-going efforts on developing a new detection system to meet the challenges. Chapter 6 presents different approaches to cool the center-of-mass motion of an optically trapped microsphere in vacuum, and the results of $3 \mathrm{D}$ optical feedback cooling. We also discuss the trapping lifetime of optically 
trapped microspheres in vacuum. Finally, chapter 7 discusses a scheme to perform 3D ground-state cooling of a optically trapped nanosphere with a single cavity, and the potential applications of cooled microspheres in vacuum. 


\section{Chapter 2}

\section{Physical principle of optical tweezers}

The radiation pressure of light was first deduced theoretically by James C. Maxwell in 1873 based on his electromagnetic theory [38, 39], and measured experimentally by P. N. Lebedev [40], and E. F. Nichols and G. F. Hull in 1901 [41]. The radiation pressure force exerted on a totally reflecting mirror by an incident beam of light perpendicular to the mirror is $F_{\text {mirror }}=2 P / c$, where $P$ is the power of the light and $c$ is the speed of light in vacuum [42]. The factor of 2 in the formula is due to reflection. The force is about $7 \mathrm{nN}$ for $1 \mathrm{~W}$ of light, which is tiny and had almost no application before the invention of the laser. In contrast to classical light sources, a laser beam can be strongly focused onto a small particle with a diameter on the order of $1 \mu \mathrm{m}$. Due to the small mass of the particle, the radiation force of a $1 \mathrm{~W}$ laser can be $10^{5}$ times larger than the gravitational force on the particle, and can therefore have huge effects on the motion of the particle.

In 1970, Arthur Ashkin published a seminal paper [43] demonstrating that one could use focused laser beams to accelerate and trap micrometer-sized transparent particles. Optical levitation of oil droplets and glass microspheres in air [44] and vacuum was demonstrated several years later [45]. The laser 
radiation pressure was soon used to cool and trap atoms [46-49], leading to dramatic breakthroughs in atomic, molecular and optical physics, including a new generation of atomic clocks, and realization of Bose-Einstein condensation and degenerate Fermi gas. In 1986, Ashkin et al. [50] observed stable trapping of dielectric particles with the gradient force of a strongly focused laser beam. This technique was soon used to trap and manipulate viruses and bacteria $[51,52]$, and became a standard tool in biophysics [42].

In this chapter, we will first explain the principle of optical trapping of microspheres with ray optics, which is valid when the size of the microspheres is much larger than the wavelength of the trapping laser. This will be followed by theoretical calculations of the optical forces on a particle with the Rayleigh approximation, and numerical results of Lorentz-Mie theory. The differences between trapping microspheres in air and in water will be discussed.

\subsection{Ray optics approximation}

When the size of a microsphere is much larger than the wavelength of the trapping laser (usually $R>10 \lambda_{0}$, where $R$ is the radius of the microsphere and $\lambda_{0}$ is the wavelength of the laser in vacuum), the optical forces on the microsphere can be calculated by ray optics [53].

A qualitative view of optical trapping of microspheres in the ray optics regime is shown in Fig. $2.1[50,53]$. If we neglect surface reflection from the microsphere, then the microsphere will be trapped at the focus of the laser beam as shown in Fig. 2.1(B). If the microsphere moves to the left of the 
focus (Fig. 2.1(A)), it will deflect the laser beam to the left and thus increase the momentum of photons to the left. The counter force from the deflected photons will push the microsphere to the right, i.e. back to the focus of the laser beam. If the microsphere moves along the propagation direction of the laser beam (Fig. 2.1(C)), it will focus the laser more strongly and thus increase the momentum of photons along the propagation direction. The counter force from the deflected photons will push the microsphere back to the focus of the laser beam. The same thing will happen if the microsphere moves away from the focus in other directions. Thus a focused laser beam forms a stable optical trap in $3 \mathrm{D}$.

The above discussion neglected surface reflection from the microsphere.

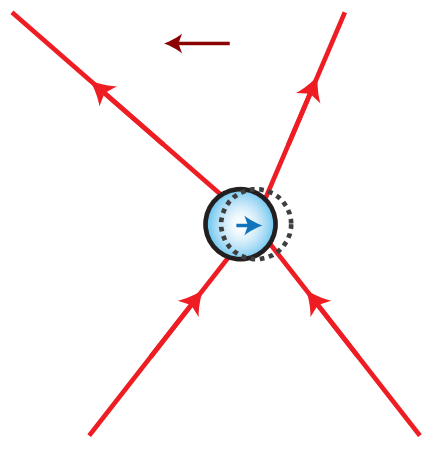

(A)

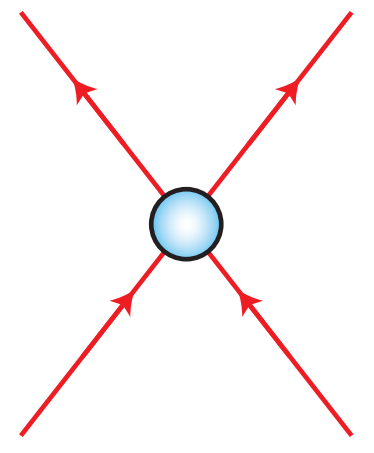

(B)

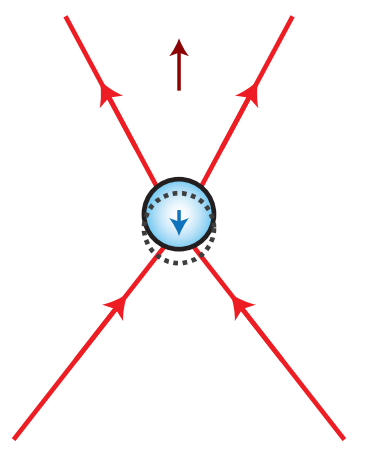

(C)

Figure 2.1: Qualitative view of optical trapping of dielectric spheres. (A) displays the force on the particle when the particle is displaced laterally from the focus; (B) shows that there is no net force on the particle when the particle is trapped at the focus; and $(\mathrm{C})$ displays the force on the particle when the particle is positioned above the focus. 
In reality, we have to consider the effect of this surface reflection. The photons reflected back by the surface of a microsphere will push the microsphere forward. If this force is larger than the restoring force due to refraction (Fig. 2.1(C)), the microsphere will be pushed away from the focus, and thus cannot be trapped. The surface reflection depends on the relative refractive index of the microsphere and the medium $m=n_{p} / n_{m d}$, where $n_{p}$ is the refractive index of the microsphere and $n_{m d}$ is the refractive index of the medium. Larger $m$ implies more surface reflection, and thus greater difficulty in trapping the microsphere with an optical tweezer [54]. $m$ is about 1.10 for a silica microsphere $\left(n_{\text {silica }}=1.46\right)$ in water $\left(n_{\text {water }}=1.33\right)$, and is about 1.46 for a silica microsphere in air $\left(n_{\text {air }}=1.00\right)$ (see Table A.1 at Appendix A for more information). Thus it is more difficult to trap microspheres in air than in water.

To increase the restoring force, the laser beam should be strongly focused by a high numerical aperture (NA) objective lens. The typical NA of objective lenses used for creating optical tweezers is about 1.2 and 0.95 in water $[50,54]$ and air [55], respectively.

\subsection{Rayleigh approximation}

If the size of a nanosphere (microsphere) is much smaller than the wavelength of the trapping laser (usually $R<\lambda_{0} / 10$ ), the nanosphere can be approximated as a dipole. In this regime, the optical force on the nanosphere can be calculated analytically with the Rayleigh scattering theory $[50,56]$. Here we will calculate the optical forces in the Rayleigh regime following the 
formulas of Ref. [56].

We consider a nanosphere with radius $R$ and a refractive index $n_{p}$ being illuminated by a laser beam propagating along the $z$ axis in the positive direction, as shown in Fig. 2.2. The power of the laser beam is $P$. The refractive index of the medium in which the nanosphere is suspended is $n_{m d}$. The laser beam is a linearly polarized Gaussian beam $\left(T E M_{00}\right)$ with beam waist radius $\omega_{0}$ at the focus. The polarization direction of the electric field of the laser is parallel to the $x$ axis. The center of the laser beam is located at the origin, and the center of the nanosphere is at $\vec{r}=(x, y, z)$.

The wavefront of a Gaussian beam is flat at the focus, and its waist $\left(1 / e^{2}\right.$ radius $)$ spreads in accordance with [57]:

$$
\omega(z)=\omega_{0}\left[1+\left(\frac{\lambda_{m d} z}{\pi \omega_{0}^{2}}\right)^{2}\right]^{1 / 2},
$$

where $\lambda_{m d}=\lambda_{0} / n_{m d}$ is the wavelength of the laser in the medium.

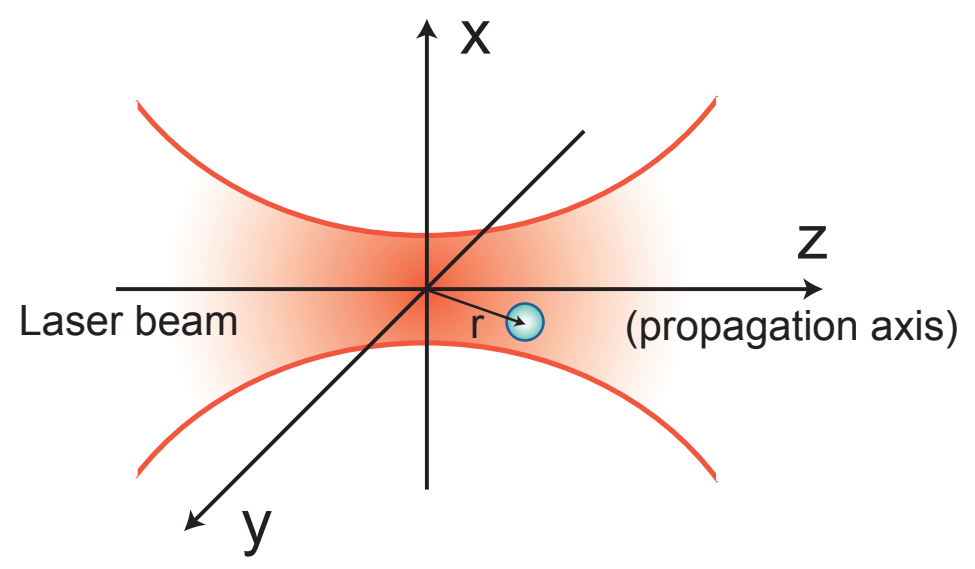

Figure 2.2: Schematic of a nanosphere near the focus of a laser beam. 
The Rayleigh range $\left(z_{R}\right)$, defined as the distance over which the beam radius spreads by a factor of $\sqrt{2}$, is given by

$$
z_{R}=\frac{\pi \omega_{0}^{2}}{\lambda_{m d}}
$$

The intensity distribution of the Gaussian beam is

$$
I(x, y)=I_{0} e^{-2\left(x^{2}+y^{2}\right) / \omega^{2}}=\frac{2 P}{\pi \omega^{2}} e^{-2\left(x^{2}+y^{2}\right) / \omega^{2}},
$$

where $\omega=\omega(z)$ and $P$ is the power of the laser beam.

The numerical aperture $\left(1 / e^{2}\right.$ points in $\mathrm{k}$-space) of a Gaussian beam is

$$
N A=\frac{\lambda_{0}}{\pi \omega_{0}}=n_{m d} \frac{\lambda_{m d}}{\pi \omega_{0}} .
$$

The optical force of the focused laser beam on the nanosphere can be separated into two components: the scattering force $\vec{F}_{\text {scat }}(\vec{r})$ which is proportional to the intensity of the laser, and the gradient force $\vec{F}_{\text {grad }}(\vec{r})$ which is proportional to the gradient of the intensity of the laser. The scattering force is a nonconservative force and the gradient force is a conservative force. The gradient force forms a trapping potential for the nanosphere, and the scattering force tends to push the nanosphere out of the trap. In order to form a stable trap, the gradient force should be lager than the scattering force.

The scattering force of the laser on a nanosphere is [56]:

$$
\vec{F}_{\text {scat }}(\vec{r})=\hat{z}\left(\frac{n_{m d}}{c}\right) C_{\text {scat }} I(\vec{r})=\hat{z} \frac{128 \pi^{5} R^{6}}{3 c \lambda_{0}^{4}}\left(\frac{m^{2}-1}{m^{2}+2}\right)^{2} n_{m d}^{5} I(\vec{r}),
$$

where $c$ is the speed of light in vacuum and $C_{\text {scat }}$ is the scattering cross section. Because of the larger relative refractive index $m$, the scattering force 
on a nanosphere in air is about 4.2 times greater than the scattering force on the same nanosphere in water with the same laser intensity. The number of scattered photons per second is

$$
N_{\text {scat }}=\frac{\lambda_{m d}}{h}\left|\vec{F}_{\text {scat }}\right|
$$

where $h$ is the Planck constant.

The gradient force on the nanopshere is [56]:

$$
\vec{F}_{\text {grad }}(\vec{r})=[\vec{p}(\vec{r}, t) \cdot \nabla] \vec{E}(\vec{r}, t)=\frac{2 \pi n_{m d} R^{3}}{c}\left(\frac{m^{2}-1}{m^{2}+2}\right) \nabla I(\vec{r}),
$$

where $\vec{p}(\vec{r}, t)$ is the induced dipole of the nanosphere due to the instantaneous electric field $(\vec{E}(\vec{r}, t))$ of the laser. The gradient force forms a trapping potential:

$$
V(\vec{r})=-\frac{2 \pi n_{m d} R^{3}}{c}\left(\frac{m^{2}-1}{m^{2}+2}\right) I(\vec{r}) .
$$

The total force on the nanosphere is $\vec{F}(\vec{r})=\vec{F}_{\text {scat }}(\vec{r})+\vec{F}_{\text {grad }}(\vec{r})$. The minimum force along the $z$ axis $F_{z}^{\text {min }}=\min \left(F_{z}(\vec{r})\right)$ must be negative in order to form a stable trap. Otherwise the force of the laser will always push the nanosphere forward and there will be no trap. Because the scattering force is proportional to $R^{6}$ while the gradient force is proportional to $R^{3}$, the scattering force decreases much faster than the gradient force when the size of the nanosphere decreases. Thus it is easier to achieve a negative $F_{z}^{\text {min }}$ for a small nanosphere than a large particle.

In order to trap a nanosphere stably, the well depth should be at least 10 times larger than the average kinetic energy of the nanosphere. This is 
due to the fact that the kinetic energy of a nanosphere follows the MaxwellBoltzmann distribution at thermal equilibrium. The nanosphere has a significant probability for its instantaneous kinetic energy to be much larger than its average kinetic energy. According to the energy equipartition theorem, the average kinetic energy of a nanosphere is $k_{B} T / 2$ in each direction, where $k_{B}$ is the Boltzmann constant and $T$ is the temperature of the medium. While the average kinetic energy is independent of the size of the nanosphere, the well depth of the trapping potential decreases as the size of the particle decreases. Thus it is difficult to trap a nanosphere if its size is too small.

Fig. 2.3 shows the calculated potentials and forces on a silica nanosphere in air with a focused laser beam. Since the refractive index of air is very close to the refractive index of vacuum, the potential and force on a nanosphere in air is practically the same as that in vacuum. For the calculations yielding Fig. 2.3, the wavelength of the laser is $1064 \mathrm{~nm}$, the power of the laser is $200 \mathrm{~mW}$, and the waist of the laser at the focus is $1.5 \mu \mathrm{m}$, corresponding to NA $=0.22$. The Rayleigh range of the laser is $6.6 \mu \mathrm{m}$. The diameter $(D=2 R)$ of the nanosphere is $50 \mathrm{~nm}$. The calculated well depth of the trap is $367 \mathrm{~K}$. The laser will therefore only be able to trap a $50 \mathrm{~nm}$ nanosphere at a temperature much lower than room temperature. The potential is approximately harmonic near the bottom of the trap. The oscillation frequency is about $42 \mathrm{kHz}$ in the radial direction and $6.7 \mathrm{kHz}$ in the axial direction for a $50 \mathrm{~nm}$ nanosphere trapped near the bottom of the potential. The scattering force is zero in the radial direction (Fig. 2.3C) and is positive along the axial direction (Fig. 2.3D). 
The gradient force is negative at positive coordinates, and positive at negative coordinates. Thus it will always pull back the nanosphere to the center of the trap.

Fig. 2.4 shows the calculated potentials and forces on a silica nanosphere in a laser beam with a much smaller waist. The waist of the laser beam is $0.5 \mu \mathrm{m}$, which corresponds to $\mathrm{NA}=0.68$. Other conditions are the same as in
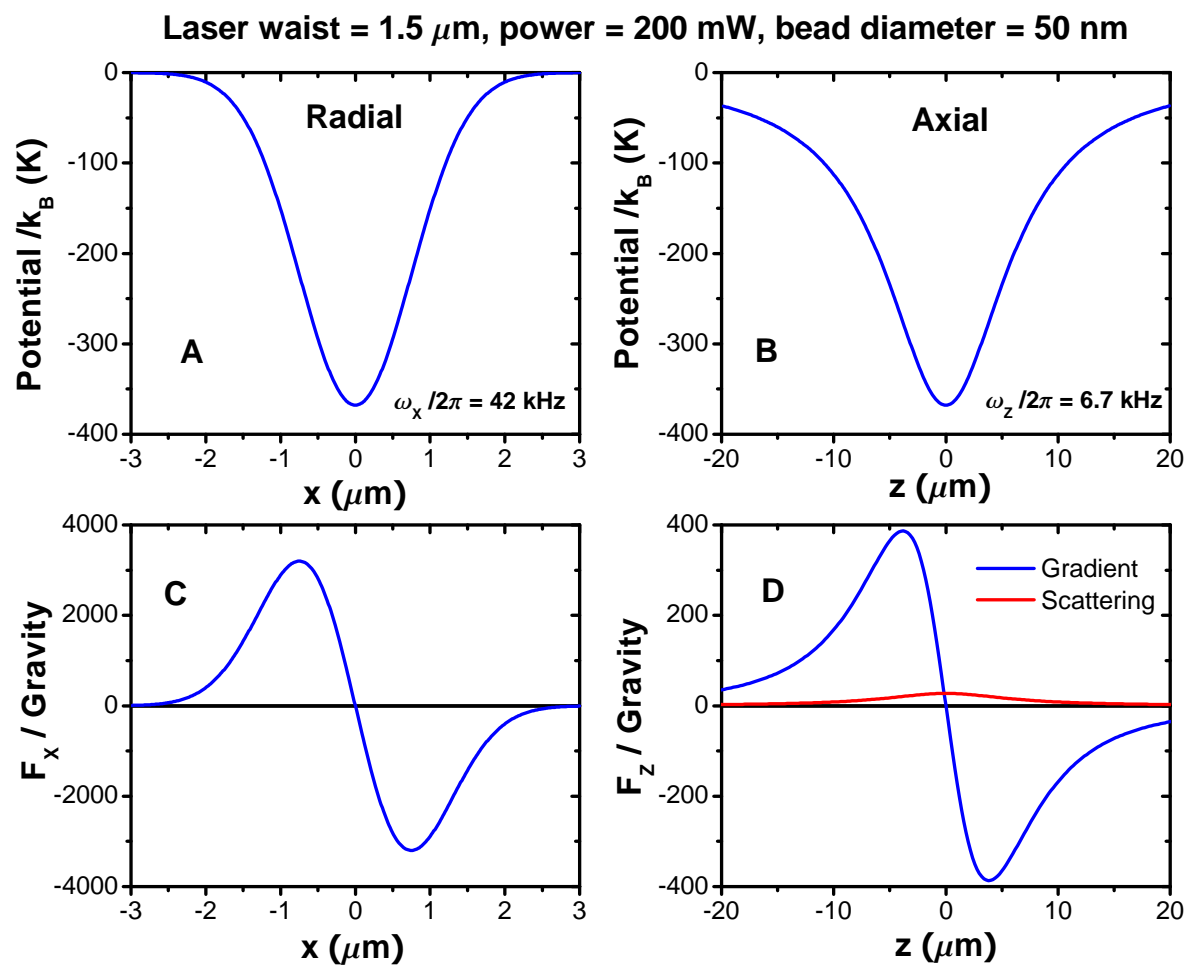

Figure 2.3: Optical potentials and forces on a nanosphere in air when the waist of the trapping laser is $1.5 \mu \mathrm{m}$. The power of the trapping laser is $200 \mathrm{~mW}$ and the diameter of the nanosphere is $50 \mathrm{~nm}$. 
Fig. 2.3. Because of the smaller waist, the well depth becomes large enough (3310 K) to trap a $50 \mathrm{~nm}$ nanosphere at room temperature. The trapping frequency is about $376 \mathrm{kHz}$ in the radial direction and $180 \mathrm{kHz}$ in the axial direction. The scattering force is negligible compared to the gradient force. Comparing Fig. 2.4 and Fig. 2.3, it is clear that a laser beam focused by an objective lens with a larger NA is much better for trapping nanospheres.
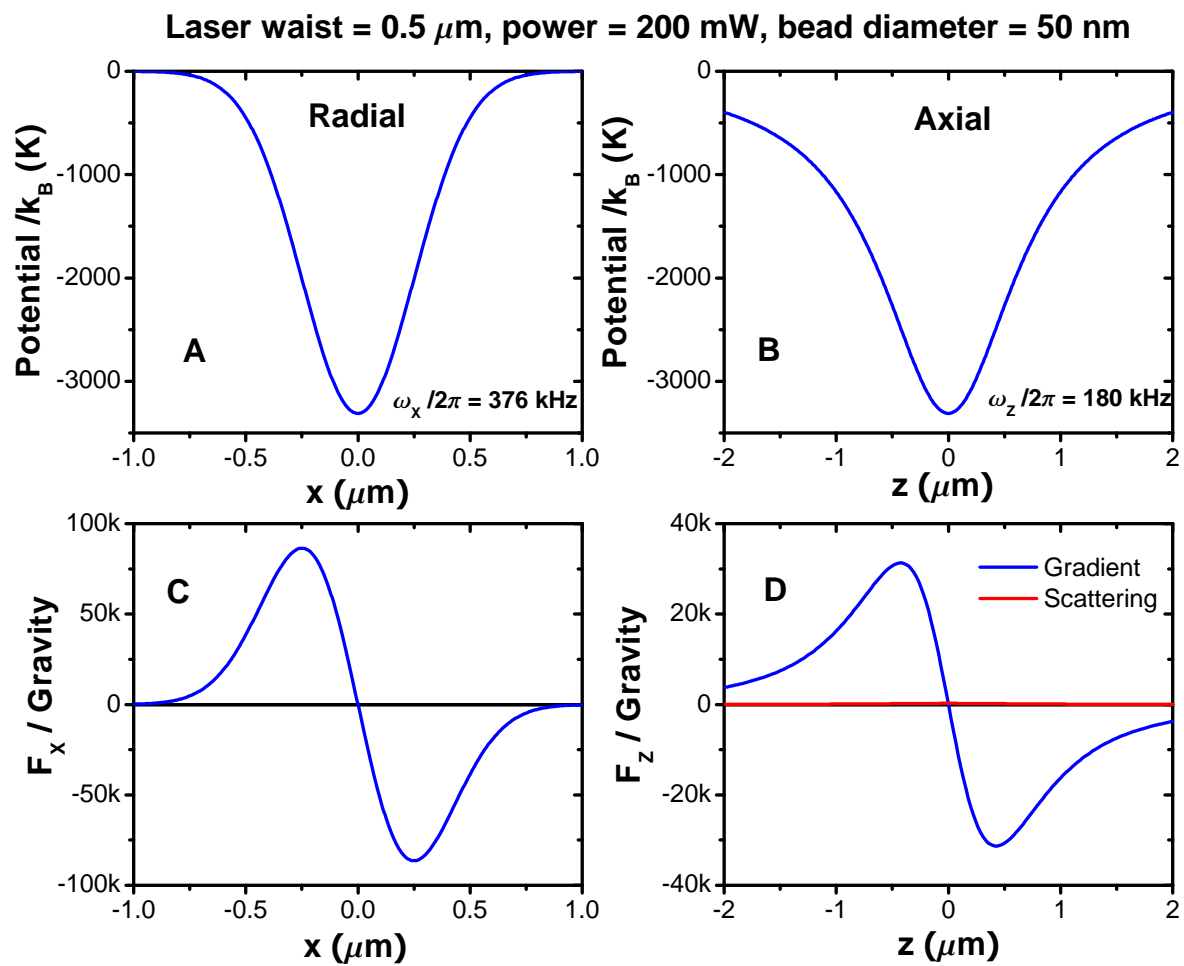

Figure 2.4: Optical potentials and forces on a nanosphere in air when the waist of the trapping laser is $0.5 \mu \mathrm{m}$. The power of the trapping laser is $200 \mathrm{~mW}$ and the diameter of the nanosphere is $50 \mathrm{~nm}$. 


\subsection{Generalized Lorentz-Mie theory}

In most experiments with optical tweezers, the sizes of the dielectric particles are comparable with the wavelength of the trapping laser $\left(R \sim \lambda_{0}\right)$. In this case, neither ray optics nor the Rayleigh approximation is appropriate. Instead the electromagnetic theory of light has to be used. For optical trapping of homogeneous and isotropic microspheres, one can use the generalized Lorenz-Mie theory. The mathematical calculation of the generalized LorenzMie theory is quite complex. Here we will only introduce this method briefly, and use the computational toolbox developed by T. A. Nieminen et al. [58] to obtain some numerical results of the optical force on a microsphere.

The optical force on a microsphere comes from the momentum of photons (electromagnetic field) from a laser. It can be obtained by calculating the change of the momentum of the electromagnetic field scattered by the microsphere. A natural choice of coordinate system for calculating the light scattering by a micropshere is spherical coordinates $(r, \theta, \phi)$ centered on the trapped microsphere. The incoming and outgoing fields can be expanded in terms of incoming and outgoing vector spherical wavefunctions [58]:

$$
\begin{aligned}
& \mathbf{E}_{\text {in }}=\sum_{i=1}^{\infty} \sum_{j=-i}^{i} a_{i j} \mathbf{M}_{i j}^{(2)}(k \mathbf{r})+b_{i j} \mathbf{N}_{i j}^{(2)}(k \mathbf{r}), \\
& \mathbf{E}_{\text {out }}=\sum_{i=1}^{\infty} \sum_{j=-i}^{i} p_{i j} \mathbf{M}_{i j}^{(1)}(k \mathbf{r})+q_{i j} \mathbf{N}_{i j}^{(1)}(k \mathbf{r}),
\end{aligned}
$$

where $\mathbf{M}_{i j}^{(1)}$ and $\mathbf{N}_{i j}^{(1)}$ are outward-propagating TE and TM multipole fields,

and $\mathbf{M}_{i j}^{(2)}$ and $\mathbf{N}_{i j}^{(2)}$ are the corresponding inward-propagating multipole fields. 
The optical force on the microsphere along the axial direction is [58]:

$$
\begin{aligned}
F_{z}= & \frac{2 n_{m d} P}{c S} \sum_{i=1}^{\infty} \sum_{j=-i}^{i} \frac{j}{i(i+1)} \operatorname{Re}\left(a_{i j}^{*} b_{i j}-p_{i j}^{*} q_{i j}\right) \\
& -\frac{1}{i+1}\left[\frac{i(i+2)(i-j+1)(i+j+1)}{(2 i+1)(2 i+3)}\right]^{1 / 2} \\
& \times \operatorname{Re}\left(a_{i j} a_{i+1, j}^{*}+b_{i j} b_{i+1, j}^{*}-p_{i j} p_{i+1, j}^{*}-q_{i j} q_{i+1, j}^{*}\right),
\end{aligned}
$$

where

$$
S=\sum_{i=1}^{\infty} \sum_{j=-i}^{i}\left(\left|a_{i j}\right|^{2}+\left|b_{i j}\right|^{2}\right) .
$$

Fig. 2.5 shows the calculated optical forces on a microsphere in water from a laser beam focused by objective lenses with three different NA's. The wavelength of the laser is $1064 \mathrm{~nm}$, the power of the laser is $100 \mathrm{~mW}$, and the diameter of the microsphere is $3 \mu \mathrm{m}$. The optical forces along the radial direction are similar for all three NA's $(\mathrm{NA}=0.85,1.0,1.25)$ as shown in Fig. 2.5B. On the other hand, the optical forces along the axial direction are very different for different NA's. This is because the scattering force is only along the axial direction. The microspheres will be trapped at positions where the total optical force changes its sign. The scattering force affects the trapping position (Fig. 2.5A).

Figure 2.6 shows the calculated optical forces on microspheres in air exerted by a laser beam focused by an objective lens with $\mathrm{NA}=0.95$. The maximum value of the NA for an objective lens in air is 1.0, while it is 1.33 for an objective lens in water. Because of a larger relative refractive index in air than in water, the scattering force on a microsphere in air is much larger than 
that in water. This makes Fig. 2.6 appear very different from Fig. 2.5. The optical forces along the axial direction are asymmetric, because the scattering forces are in the forward direction.

Figure 2.7 shows more calculation results of optical forces on microspheres in air along the axial direction. For a $D=3.0 \mu \mathrm{m}$ microsphere (Fig. $2.7 \mathrm{~A})$, the minimum axial force $\left(F_{z}^{\min }\right)$ is positive when $\mathrm{NA}=0.85$ or $\mathrm{NA}=$
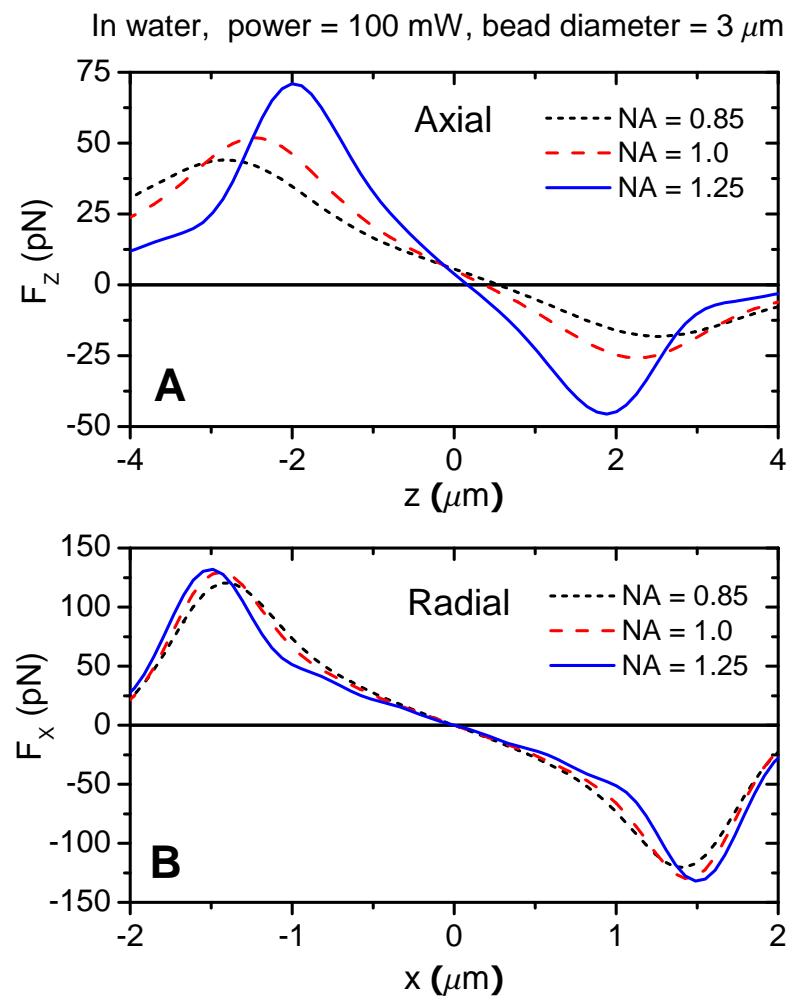

Figure 2.5: Optical forces on a microsphere in water along axial $(\mathbf{A})$ and radial (B) directions of a laser beam focused by objective lenses with different numerical apertures (NA's). The power of the laser is $100 \mathrm{~mW}$, and the diameter of the microsphere is $3 \mu \mathrm{m}$. 
0.9 , and is only slightly negative when $\mathrm{NA}=0.95$. Thus a laser beam focused by an objective lens with NA less than 0.95 can not trap a $3-\mu \mathrm{m}$ silica microsphere. The situation becomes better for smaller microspheres. The minimum axial force is negative for a $0.5-\mu \mathrm{m}$ microsphere in a laser beam focused by objective lenses with all three different NA's (Fig. 2.7D).

The minimum axial forces on a microsphere in air as a function of the
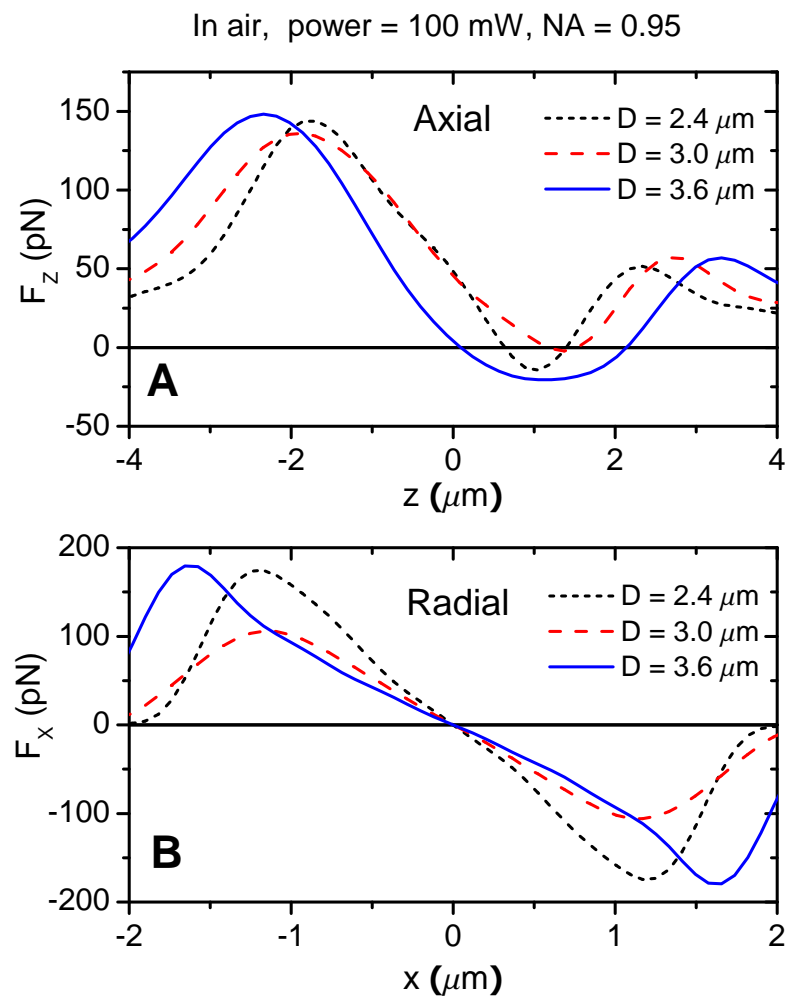

Figure 2.6: Optical forces on microspheres in air along axial (A) and radial (B) directions of a laser beam focused by an objective lens with NA $=0.95$. The power of the laser is $100 \mathrm{~mW}$. The diameter of the microsphere is $2.4 \mu \mathrm{m}$ for the dotted lines, $3.0 \mu \mathrm{m}$ for the dashed lines, and $3.6 \mu \mathrm{m}$ for the solid lines. 
diameter of the microsphere are shown in Figure 2.8. The minimum forces oscillate as the diameter of the microsphere changes. This is because of the interference between the scattered light and un-scattered light. The oscillation period is about half the wavelength of the laser inside of the microsphere, which is $\lambda /\left(2 n_{p}\right)=364 \mathrm{~nm}$. A microsphere can not be trapped if the minimum force is positive. For NA $=0.85$, only microspheres with certain diameters can be trapped. This serves as a selection process and can be used for sorting mi-
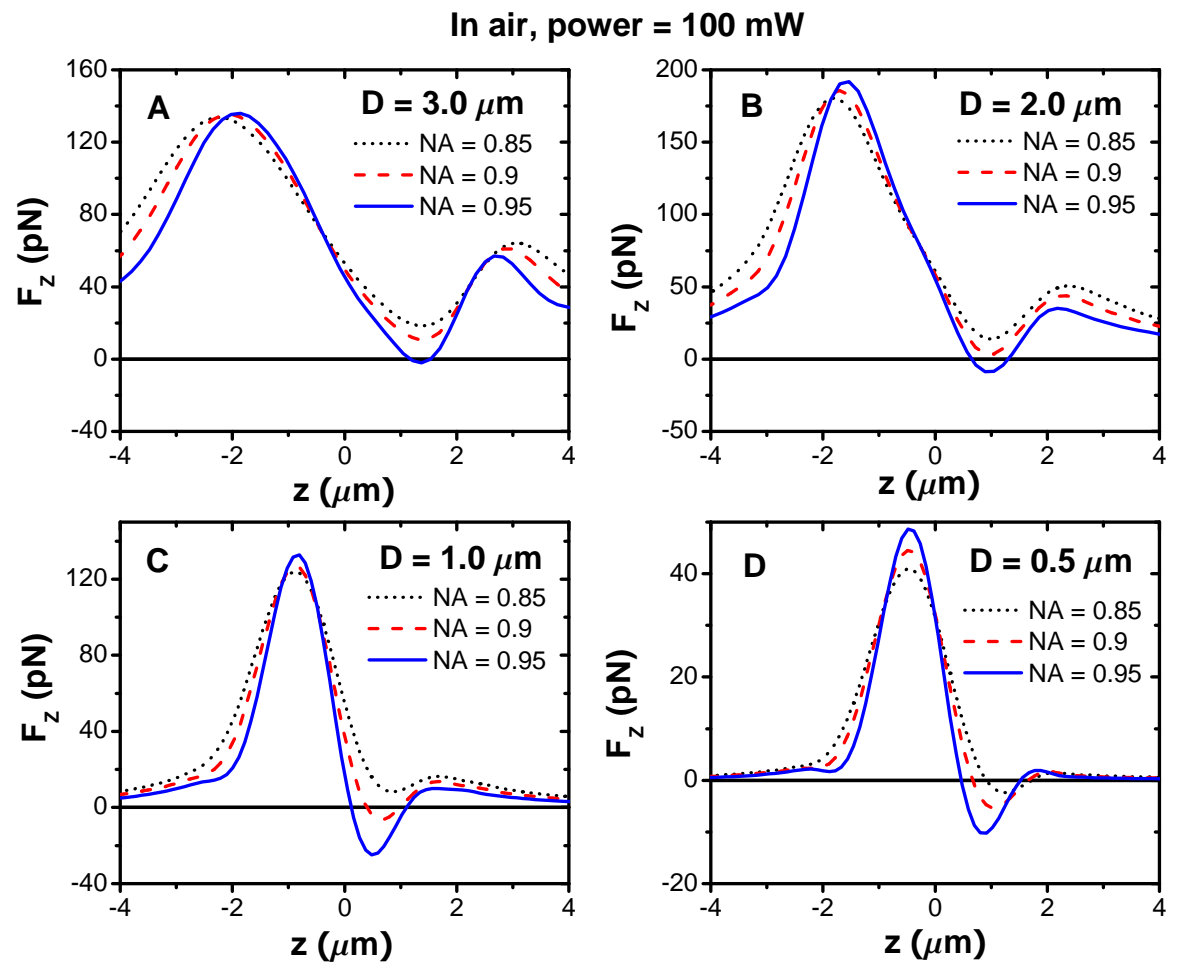

Figure 2.7: Optical forces on microspheres in air as a function of the diameter of the microspheres and the NA of laser beams. 
crospheres. The size distribution of the trapped microspheres will be different from the size distribution of the microspheres before trapping. For example, if the microspheres prior trapping have a large diameter distribution ranging from $0.7 \mu \mathrm{m}$ to $1.7 \mu \mathrm{m}$, the diameter of microspheres trapped by a $\mathrm{NA}=0.85$ laser will always be about $1.4 \mu \mathrm{m}$.

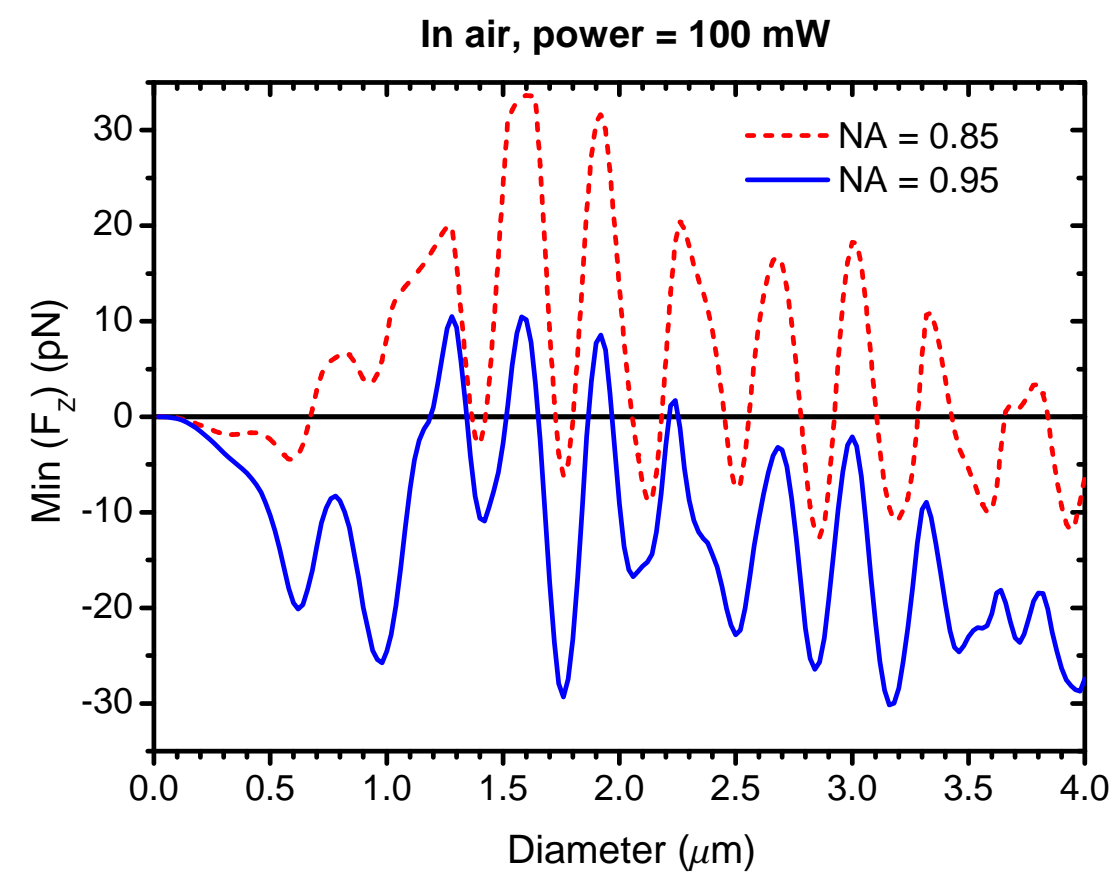

Figure 2.8: Minimum optical forces on a microsphere in air along the axial direction as a function of the diameter of the microspheres. The power of the laser is $100 \mathrm{~mW}$. 


\section{Chapter 3}

\section{Optical trapping of glass microspheres in air and vacuum}

Optical levitation of dielectric particles in air by an upward-propagating laser beam was first demonstrated by A. Ashkin and J. M. Dziedzic in 1971 [44]. A few years later, optical levitation of microspheres in vacuum at pressures down to $10^{-6}$ torr was achieved [45]. An optical levitation trap is formed by the balance between the scattering force from an upward laser and the gravitational force on a particle. The trapping frequency of an optical levitation trap is usually very small (about $20 \mathrm{~Hz}$ ) [45]. Optical trapping of a dielectric particle in air with the gradient force of a single laser beam was first demonstrated in 1997 [55], 11 years after the invention of the single beam gradient force optical trap in water [50]. So far there is still no report on optical trapping of a microsphere in vacuum by a single beam gradient force trap (without the help of the gravitational force).

Optical trapping of a microsphere in air with the optical gradient force is much more difficult than optical trapping of a microsphere in water [59]. First, the scattering force on a microsphere from a laser beam is much larger in air than that in water, because of the larger relative refractive index of a 
microsphere in air $(m=1.46)$ than that in water $(m=1.1)$. There will be no stable optical trap if the scattering force is larger than the gradient force, as discussed in detail in Chapter 2. Second, microspheres in air will fall rapidly due to the influence of gravity. On the other hand, microspheres in water can be suspended for a long time. In air, we have to launch a lot of microspheres to air first and capture one of them by an optical trap passively. In water however, we can move a microsphere to the focus of a laser beam (by moving the water chamber with a translation stage) and trap it actively.

In this chapter we will first describe the method used to launch microspheres into air; we will then show our results of trapping microspheres in air and vacuum. Finally, we will describe the structure of our vacuum system.

\subsection{Launching microspheres}

The silica $\left(\mathrm{SiO}_{2}\right)$ microspheres (bought from Bangs Laboratories, Inc.) are initially stuck on a glass surface and with each each other by the van der Waals' (attractive) force. The minimum required force to pull a microsphere off from a surface is called the "pull-off force". The pull-off force between a glass microsphere and a flat glass surface is predicted by the model of Derjaguin, Muller, and Toporov (DMT) to be [60,61]:

$$
F_{\text {sphere-flat }}=4 \pi R \gamma
$$


where $R$ is the radius of the microsphere and $\gamma$ the effective solid surface energy. The pull-off force between two identical glass microspheres is

$$
F_{\text {sphere-sphere }}=2 \pi R \gamma
$$

which is half of the pull-off force between a microsphere and a flat surface.

The pull-off force between two 1- $\mu$ m-diameter silica microspheres is measured to be about $88 \mathrm{nN}$ [61], and the pull-off force between a 1- $\mu \mathrm{m}$ diameter silica microsphere and a flat silica surface is about $176 \mathrm{nN}$. The gravitational force on a $1-\mu \mathrm{m}$-diameter silica microsphere is only about $10 \mathrm{fN}$, and the maximum force from a typical optical tweezer is about $0.1 \mathrm{nN}$. Thus the gravitational force and a typical optical force are too small to pull off a microsphere from a glass surface. In our experiment, we launch microspheres to air by ultrasonic vibration. The required acceleration to break the van der Waals' bond between a microsphere and a flat surface is:

$$
a=\frac{F_{\text {sphere }- \text { flat }}}{M}=\frac{4 \pi R \gamma}{\frac{4}{3} \pi R^{3} \rho_{\text {bead }}} \propto \frac{1}{R^{2}},
$$

where $M$ is the mass of the microsphere and $\rho_{\text {bead }}$ the density of the microsphere. The smaller the microsphere, the larger acceleration is required to break the van der Waals bond. For a 1- $\mu$ m-diameter silica microsphere, the required acceleration is about $1.8 \times 10^{8} \mathrm{~m} / \mathrm{s}^{2}$ !

Because the pull-off force between two microspheres is only half of the pull-off force between a microsphere and a flat surface, the microspheres will be separated from each other before being pulled off from a glass surface by 
ultrasonic vibration. Thus most of particles launched out by ultrasonic vibration are single microspheres, even if the microspheres are not mono-dispersed initially. This is a very good property for us, because we want to trap only a single microsphere at a time.

We launch single microspheres into air by a home-built ultrasonic transducer (Fig. 3.1A, also see Fig. 3.7 for a schematic of the ultrasonic generator). The transducer is a sandwich structure with a piezoelectric ring and a glass slide clamped together by two copper plates. The main component of the transducer is the piezoelectric ceramic ring with an outer diameter of $38 \mathrm{~mm}$, an inner diameter of $13 \mathrm{~mm}$ and a thickness of $6.35 \mathrm{~mm}$. It is made of lead zirconate / lead titanate (PZT) ceramic (APC International Ltd. ). The two
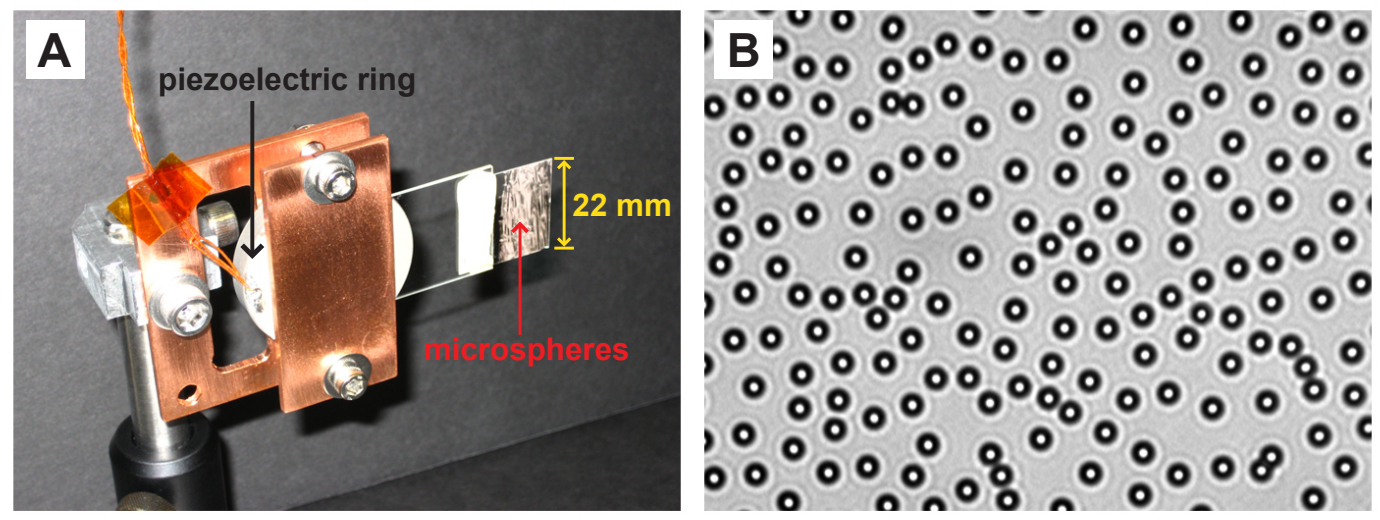

Figure 3.1: (A) A home-built ultrasonic transducer for launching glass microspheres into air. It has a sandwich structure consisting of a piezoelectric ring and a glass slide between a pair of copper plates. The microspheres are held on the surface of a coverslip by the van der Waals force. (B) Glass microspheres distributed on the surface of a glass coverslip after ultrasonic vibration. The diameter of the microspheres is $3.0 \mu \mathrm{m}$. The picture was taken under a $40 \mathrm{x}$ objective lens. 
flat surfaces of the piezoelectric ring are coated by silver layers that serve as two electrodes. One flat surface is in direct contact with a copper plate that is grounded. The other flat surface is isolated from the other copper plate by the glass slide whose thickness is about $1 \mathrm{~mm}$. A vacuum compatible kapton coated copper wire is soldered to this flat surface for applying a high voltage to the piezoelectric ring.

A No. 2 coverslip (thickness: $0.19-0.25 \mathrm{~mm}$ ) is attached to one end of the glass slide by an ultrahigh-vacuum compatible epoxy (Epoxy Technology, Inc. Model: EPO-TEK H77). The two-component epoxy becomes a hard ceramic after mixing and curing at $120{ }^{\circ} \mathrm{C}$ for 2 hours. After curing, it can be used continuously at temperatures as high as $250{ }^{\circ} \mathrm{C}$ without bond failure. The epoxy ceramic is as hard as the glass slide so it will not reduce the mechanical quality of the transducer. The purpose of using a coverslip attached to the glass slide is to increase the vibration amplitude. The microspheres are distributed on the coverslip at the side that is facing the piezo. We found that the two sides of the coverslip behave very differently for launching microspheres. We have tested several different types of ultrasonic transducers and find that this

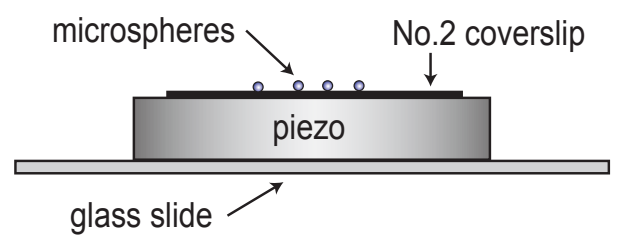

Figure 3.2: A much simpler, but less efficient ultrasonic transducer for launching glass microspheres into air. 
transducer is the best one among those we have tested.

A much simpler, but less efficient ultrasonic transducer for launching glass microspheres into air is shown in Fig. 3.2. A glass slide and a glass coverslip are attached to the two sides of a piezoelectric ring with an epoxy ceramic. The microspheres are applied on top of the coverslip. This ultrasonic transducer needs 2-4 times more ultrasonic power to launch microspheres than the transducer shown in Fig. 3.1A. However, this is not a problem for launching microspheres that are larger than $3 \mu \mathrm{m}$. This transducer is compatible with a commercial optical microscopes. The whole transducer can be mounted on a microscope stage like a glass slide. The piezoelectric ring has a hole at the center for optical access. We studied the launching process with this ultrasonic transducer before switching to more complex transducers. Photos in Fig. 3.1B and Fig. 3.3 were taken with this transducer under a commercial microscope.
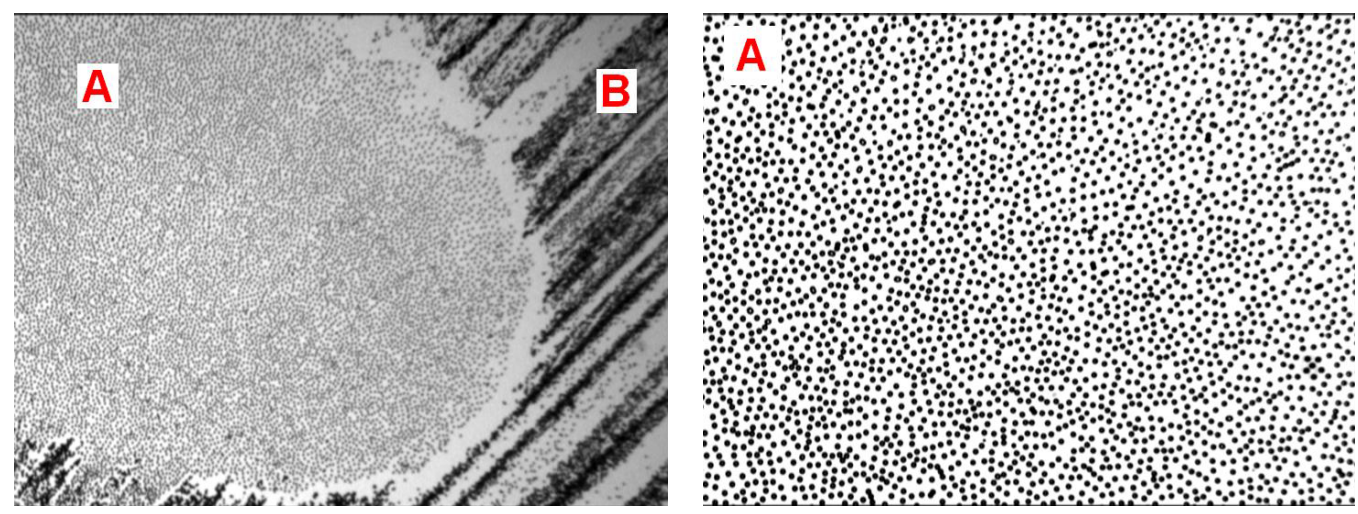

Figure 3.3: Glass microspheres distributed on the surface of a glass coverslip after ultrasonic vibration. The mean diameter of the microspheres is $3.0 \mu \mathrm{m}$. The left photo was taken under a $4 \mathrm{x}$ objective lens, and the right photo was taken under a 10x objective lens, of the region labeled ' $\mathrm{A}$ '. 
We apply dry silica microspheres directly on the coverslip of an ultrasonic transducer. If the microspheres are supplied in aqueous solution, we pipet a few drops of the aqueous solution onto a glass slide. The glass slide is heated up to about $70{ }^{\circ} \mathrm{C}$ for several minutes to get rid of water. We then scratch some microspheres from the dried glass slide and apply them to the coverslip of an ultrasonic transducer. The microspheres should be dry when they are applied to the coverslip. We find that microspheres deposited on a coverslip directly from a solution (water or acetone) cannot be launched $[62,63]$.

The required ultrasonic power for launching particles depends strongly on the size of the particles. We can first remove large clusters of microspheres by applying low ultrasonic power for a few seconds. When the ultrasonic power is large enough, the bonds between different microspheres are broken and the microspheres become mono-dispersed. During ultrasonic vibration, a standing wave is formed on the coverslip. Some regions of the coverslip will have enough vibration to launch microspheres while other regions of the coverslip do not have enough vibration to break the van der Waals' bonds.

Fig. 3.3 shows glass microspheres distributed on the surface of a coverslip after ultrasonic vibration. The mean diameter of the microspheres is $3.0 \mu \mathrm{m}$. In the left photo of the figure, we can observe two distinct regions. In region ' $\mathrm{A}$ ', microspheres are mono-dispersed because the ultrasonic vibration is large enough to break the van der Waals' bonds and redistribute the microspheres. In region 'B', microspheres are not mono-dispersed because the 
vibration in this regime is too small.

Magnified photos of microspheres in region ' $\mathrm{A}$ ' are shown in the right photo of Fig. 3.3 and Fig. 3.1B. They show that most microspheres are isolated from each other after ultrasonic vibration, thus most of the launched particles are single microspheres. The launched spheres fall under gravity, and eventually a sphere approaches the laser focus and is trapped. A coverslip fully covered with a monolayer of microspheres can be used to launch and trap single microspheres several hundred times before there are too few beads left.

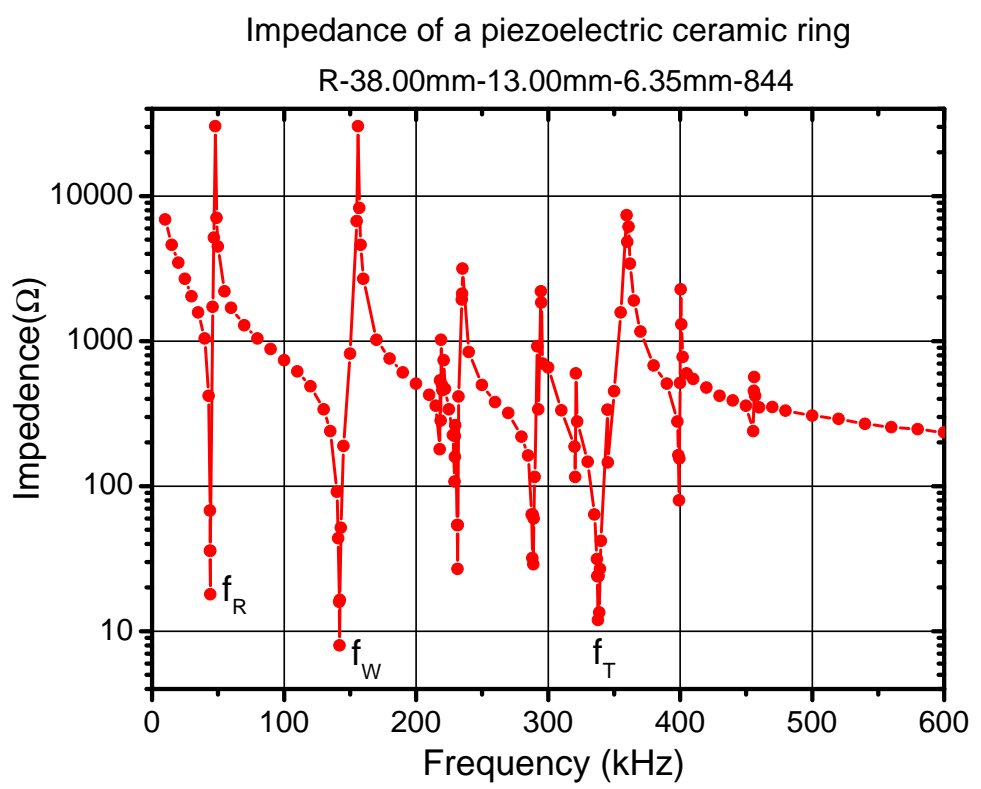

Figure 3.4: Impedance of a piezoelectric ceramic ring. The outer diameter of the ring is $38 \mathrm{~mm}$, the inner diameter is $13 \mathrm{~mm}$, and the thickness is 6.35 $\mathrm{mm}$. The material is a specially formulated, high purity lead zirconate / lead titanate (PZT) ceramic (APC 844) from APC International Ltd. It is a hard ceramic suitable for high power applications. 


\begin{tabular}{|c|c|c|}
\hline Diameter & Mass & Ultrasonic power \\
\hline \hline $4.7 \mu \mathrm{m}$ & $1.1 \times 10^{-13} \mathrm{~kg}$ & $1.3 \mathrm{~W}$ \\
\hline $3.0 \mu \mathrm{m}$ & $2.8 \times 10^{-14} \mathrm{~kg}$ & $4.2 \mathrm{~W}$ \\
\hline $1.9 \mu \mathrm{m}$ & $7.2 \times 10^{-15} \mathrm{~kg}$ & $80 \mathrm{~W}$ \\
\hline $1.5 \mu \mathrm{m}$ & $3.5 \times 10^{-15} \mathrm{~kg}$ & $133 \mathrm{~W}$ \\
\hline $1.0 \mu \mathrm{m}$ & $1.0 \times 10^{-15} \mathrm{~kg}$ & $\begin{array}{c}\text { Above } 500 \mathrm{~W} \\
\text { the piezo ring starts to break }\end{array}$ \\
\hline
\end{tabular}

Table 3.1: The masses of silica microspheres with different diameters and the required ultrasonic powers for launching them. The driving frequency is about $340 \mathrm{kHz}$. The ultrasonic powers shown here are nominal values. The real required powers also depend on the air pressure and number of microspheres remaining on the coverslip.

Since a microsphere can be trapped for many hours, we only need to reapply microspheres every few months.

Fig. 3.4 is the measured impedance of a free piezoelectric ceramic ring driven by a sine wave. We have also measured the impedance of piezoelectric rings mounted in the ultrasonic generators (Fig. 3.1, 3.2). The measured impedances are almost the same as the one shown in Fig. 3.4. As shown in Fig. 3.4, the impedance has many resonance dips corresponding to different modes of ultrasonic vibrations [64]. The circumference vibration mode (expansion of the ring circumference) has a resonant frequency of $44 \mathrm{kHz}$ and impedance of about $17.9 \Omega$ at resonance. The mechanical quality factor $(\mathrm{Q})$ of this mode is 362. The thickness vibration mode has a resonant frequency of about $340 \mathrm{kHz}$ and impedance of about $12 \Omega$ at resonance. The $\mathrm{Q}$ of this mode is 402 . We use this mode for launching microspheres to air.

In order to achieve high acceleration of the coverslip without breaking 
the ultrasonic transducer, we drive the transducer with a high power square wave at $340 \mathrm{kHz}$ for a short duration (about $10 \mathrm{~ms}$ ). A square wave causes much larger acceleration than a sine wave (the real shape of our driving wave is very complex because the piezoelectric ring has a large capacitance and inductance). The ultrasonic power required for launching 3.0- $\mu \mathrm{m}$ diameter microspheres is about $4 \mathrm{~W}$, and it is about $130 \mathrm{~W}$ for $1.5-\mu \mathrm{m}$ diameter microspheres (Table 3.1). These are nominal values. The required power also depends on air pressure and density of microspheres remaining on the slide. The damage threshold of the piezoelectric ceramic ring and the coverslip is several hundred watts. Thus we are only able to launch microspheres with diameter larger than $1 \mu \mathrm{m}$ in the present experiment.

We drive the ultrasonic transducer with a home-built high-power pulsed generator (Fig. 3.5). Since the resonant impedance of the piezoelectric ring is only about $12 \Omega$, we need to drive it with a large current to achieve high

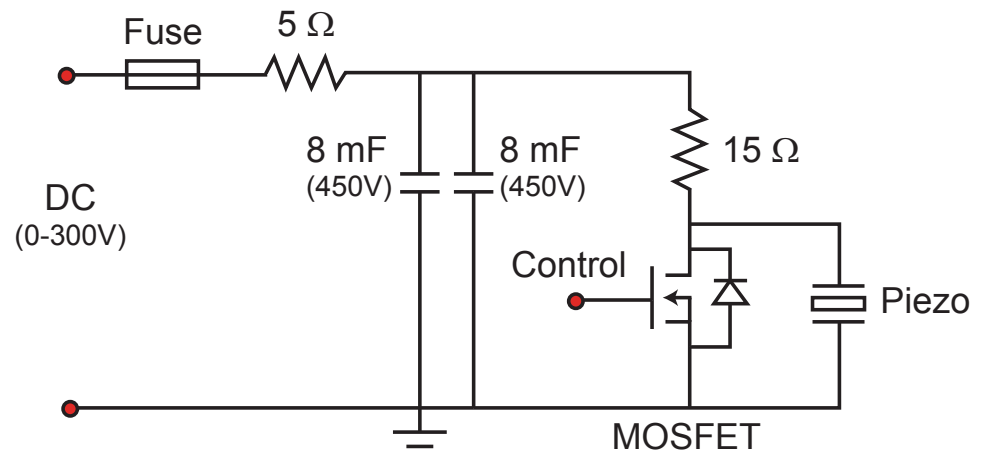

Figure 3.5: A simplified electronic circuit of our high-power pulsed generator. The power MOSFET is IRFPS40N50L from Vishay Siliconix. 
ultrasonic power. The main components of the pulsed generator are two $8 \mathrm{mF}$ capacitors (Digi-key, Model: 338-1236-ND) to store energy, and a power MOSFET to generate pulses. The limit voltage of the capacitors is $450 \mathrm{~V}$. The capacitors are connected to an external power supply which charges them. We change the output voltage of that external power supply to regulate the charge on the capacitors.

The power MOSFET is IRFPS40N50L (Vishay Siliconix). The limit voltage and current of the MOSFET are $500 \mathrm{~V}$ and $46 \mathrm{~A}$, respectively. Its "on" gate-source voltage is $10 \mathrm{~V}$, and its "on" resistance is $0.087 \Omega$. This power MOSFET has a very large input capacitance $(8110 \mathrm{pF})$, so we cannot drive it directly by a signal generator. We use a high-current high-speed MOSFET

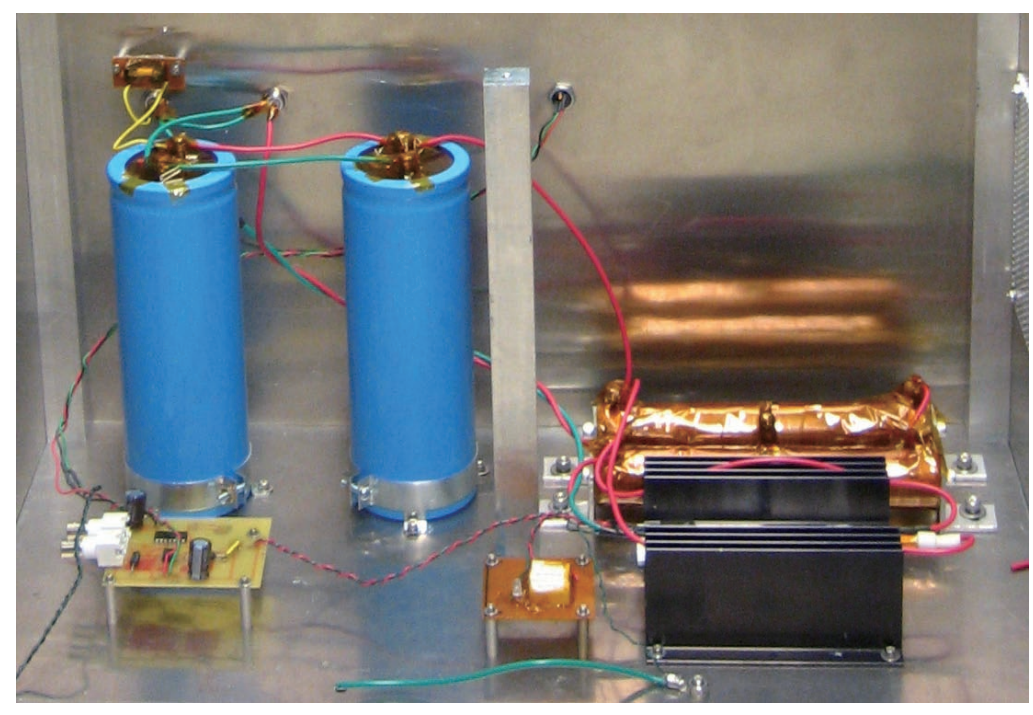

Figure 3.6: A home-built high power pulsed generator. The two blue cylinders are capacitors $(8 \mathrm{mF}, 450 \mathrm{~V})$ for storing energy to generate the high power electronic pulses. The diameter of each capacitor is $7.6 \mathrm{~cm}$, and the height is $22 \mathrm{~cm}$. 
driver (Microchip: TC4422) to drive it through the "Control" terminal in Fig. 3.5. The TC4422 has a peak output current of $9 \mathrm{~A}$ and can drive the power MOSFET at the required speed $(340 \mathrm{kHz})$. The TC4422 itself is driven by a signal generator (Agilent 33250A) that can generate arbitrary waveforms from DC to $80 \mathrm{MHz}$. The whole system is controlled by a digital output from a computer. Since the Q of the piezoelectric ring is about 402, it is enough to drive the system with pulses at $340 \mathrm{kHz}$ for $10 \mathrm{~ms}$ (about 3400 total pulses) to achieve maximum acceleration.

A photo of our high-power pulse generator is shown in Fig. 3.6.

\subsection{Trapping microspheres}

Once microspheres are launched into air, they will fall under the influence of gravity. We can capture a microsphere with an optical trap when the microsphere passes near the optical trap. Because of air damping, a microsphere that goes into an optical trap will lose its kinetic energy and becomes trapped. After a microsphere is trapped, we can reduce the air pressure and keep the microsphere trapped in vacuum.

Optical trapping of dielectric particles in air with a single beam gradient force trap was first demonstrated in 1997 [55], requiring an objective lens with $\mathrm{NA}=0.95$. So far it is still not feasible to trap a microsphere in vacuum with a single beam gradient force trap, due to the lack of vacuum-compatible objective lenses with NA of about 0.95 . 
The simplest way to trap a microsphere in air and vacuum is using an optical levitation trap, which utilizes the gravitational force to assist trapping [44]. However, the optical levitation trap is not very stable and its trapping frequency is very small (on the order of $10 \mathrm{~Hz}$ ). Thus it is not suitable for quantum ground-state cooling. We eventually decided to use a counter-propagating dual-beam gradient force trap to trap microspheres in air and vacuum. In the following sections, we will describe our experimental apparatus and results of trapping microspheres with an optical levitation trap and a dual-beam trap.

\subsubsection{Optical levitation trap}

The first optical trap that we used to trap microspheres in air was an optical levitation trap. We used a diode pumped solid-state CW laser (Coherent Inc. model: Verdi V10) for trapping. Its wavelength is $532 \mathrm{~nm}$, and its maximum power is $10 \mathrm{~W}$. As shown in Fig. 3.7, a $532 \mathrm{~nm}$ laser beam with size of about $1 \mathrm{~cm}$ is focused by an achromatic lens whose focal length is $35 \mathrm{~mm}$. The focal point of the laser beam is inside a glass cell. The width of the glass cell is about $1.0 \mathrm{~cm}$ and the height is about $5 \mathrm{~cm}$. The thickness of the walls of the glass cell is about $1 \mathrm{~mm}$. The silica microspheres are initially stuck on a coverslip above the glass cell. The gap between the coverslip and the glass cell is very small $(\sim 0.5 \mathrm{~mm})$. Thus the coverslip and the glass cell form an almost sealed chamber to protect the optical trap from air flows.

We use the ultrasonic transducer to launch the microspheres to air. After launching, the microspheres fall down due to the gravitational force. 
The air will exert a frictional force on the microspheres, $F_{\text {air }}=6 \pi \eta_{\text {air }} R \cdot v$, where $\eta_{\text {air }}$ is the viscosity of air (see Table A.1 for the values) and $v$ is the velocity of the microsphere. The terminal speed of a microsphere is

$$
v=\frac{M g}{6 \pi \eta_{\text {air }} R}
$$

where $M$ is the mass of the microsphere, and $g$ the gravitational acceleration on the Earth. The settling speed is about $0.22 \mathrm{~mm} / \mathrm{s}$ for a $1.9 \mu \mathrm{m}$ diameter silica bead, $0.57 \mathrm{~mm} / \mathrm{s}$ for a $3.0 \mu \mathrm{m}$ diameter silica bead, and $1.4 \mathrm{~mm} / \mathrm{s}$ for a $4.7 \mu \mathrm{m}$ diameter silica bead. So it takes about 3 minutes for a $1.9 \mu \mathrm{m}$ microsphere to reach the optical trap after launching. Once a microsphere is near the focus of the laser beam, it will be captured by the laser beam. In the vertical direction,

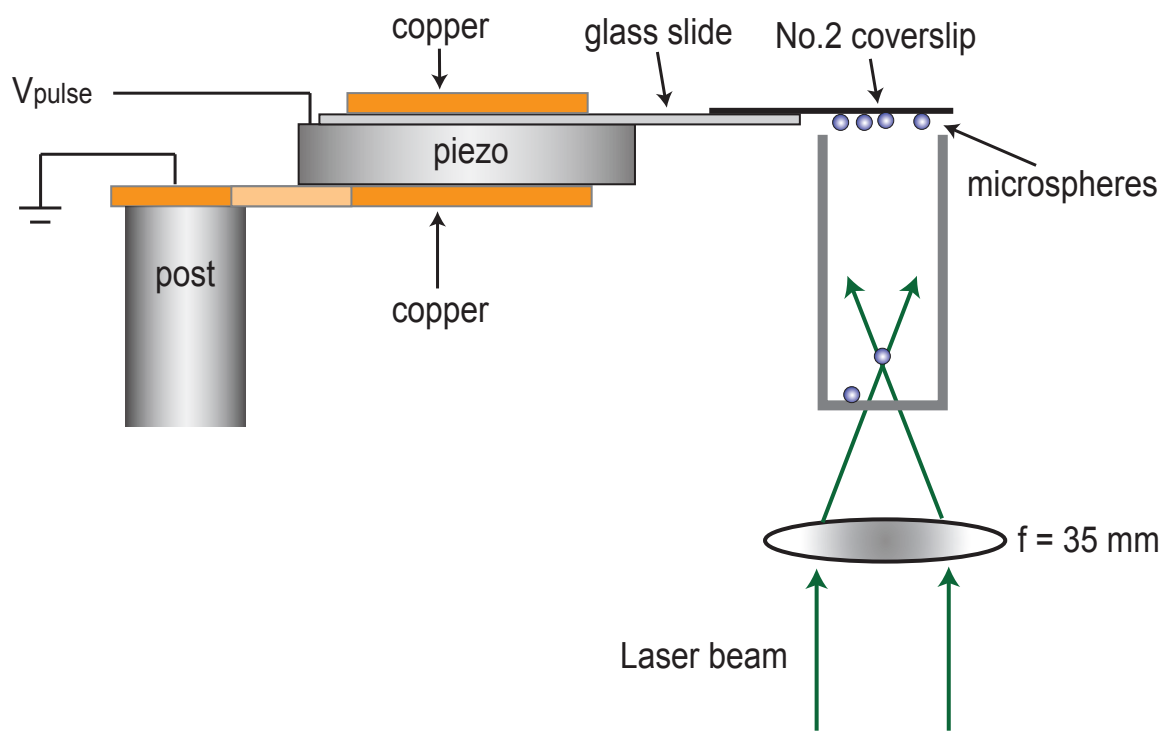

Figure 3.7: A schematic of an optical levitation trap and an ultrasonic transducer for launching microspheres. The trap is formed by the balance between the scattering force from an upward laser beam and the gravitational force on the microsphere. 
the trap is formed by the balance between the scattering force from the upward laser beam and the gravitational force on the microsphere; in the horizontal direction, the trap is formed by the gradient force from the laser beam.

A photo of a $4.7-\mu \mathrm{m}$ diameter microsphere levitated by a laser beam in air is displayed in Fig. 3.8. We can change the laser power from $2 \mathrm{~W}$ to $0.4 \mathrm{~W}$ while keeping the microsphere trapped. As expected, the vertical position of a trapped microsphere changes when we change the laser power. When we increase the laser power, the trapped microsphere moves up. Surprisingly, we found that we could also change the vertical position of a trapped microsphere

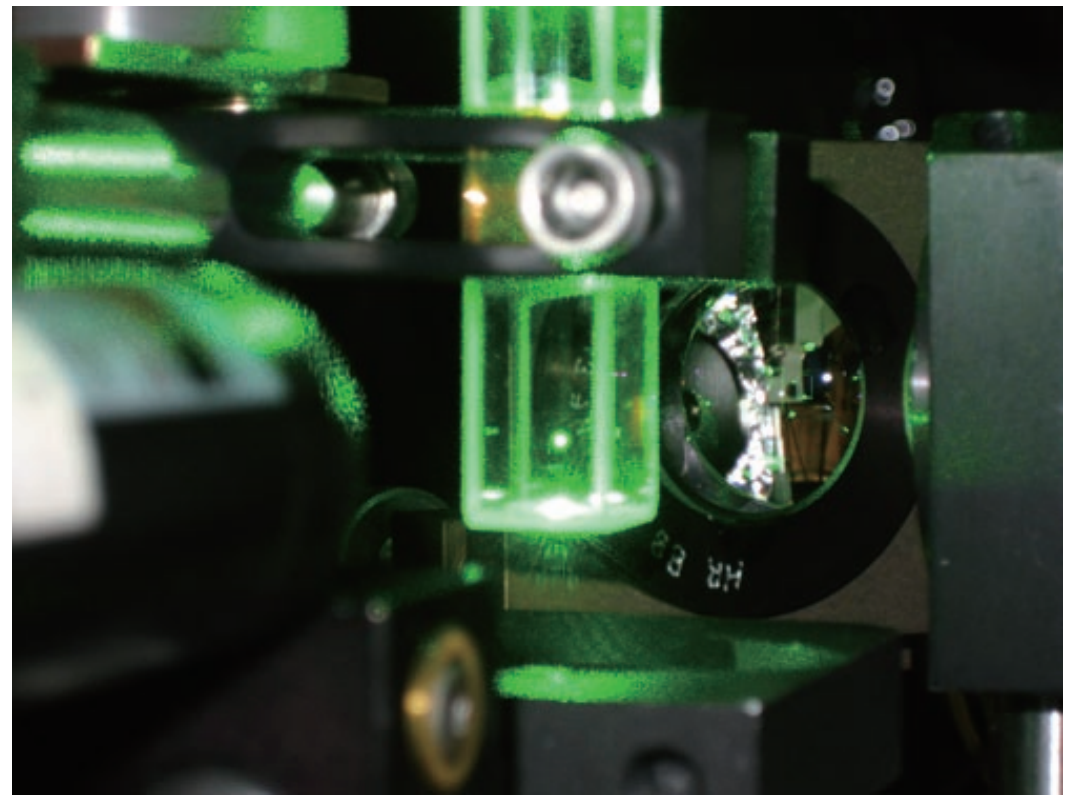

Figure 3.8: A 4.7- $\mu \mathrm{m}$ diameter silica microsphere levitated in air inside a glass cell by an upward laser beam. The bright dot near the center of the photo is the trapped microsphere. It appears much larger than the real size of the microsphere because of the overexposure of the camera. 
by driving the ultrasonic transducer. By driving with different ultrasonic frequencies, we could drag the microsphere either up or down. This suggests that the pressure force from the ultrasonic wave may be enough to levitate the microsphere. We have not studied this phenomenon in detail.

The optical levitation trap is pretty simple. However, it has several drawbacks. First, the laser beam must be upward. This is a problem because thousands of microspheres must be launched into air for each microsphere which is trapped. Those microspheres will fall to the bottom of the glass cell. They will be in the path of the laser beam and make the optical trap unstable. We can only launch and trap microspheres a few times before the bottom of the glass cell becomes too dirty. Second, the optical levitation trap tends to trap several microspheres at one time [65]. It is very difficult to trap only one microsphere at a time, or control the number of the trapped microspheres. Third, the trapping frequency of the optical levitation trap is too small. It is usually on the order of $10 \mathrm{~Hz}$ [45]. This is very close to the mechanical vibration frequencies of experimental instruments, and can cause heating of the motion of a trapped microsphere. It is important that the trapping frequency be much higher than the frequencies of seismic vibration in order to achieve significant cooling.

\subsubsection{Counter-propagating dual-beam optical trap}

The ideal optical trap for our experiment will be a single beam gradient

force trap. However, it requires an objective lens with a NA of about 0.95, 
which is currently not available for use in vacuum. A high NA objective lens usually has a very short working distance (usually about $200 \mu \mathrm{m}$ ), which is not good for us. We need the working distance to be long enough to allow optical accesses for feedback cooling and cavity cooling.

On the other hand, a low NA lens usually has a long working distance but can not focus a laser beam strongly enough to create a gradient force trap. The scattering force from a weakly focused laser will be larger than the gradient force. One way to overcome this problem is using two counterpropagating laser beams (Fig. 3.9). The scattering forces from the two beams cancel, and the gradient forces forms a stable 3D trap for the microsphere.

We built a simple dual-beam optical trap (Fig. 3.9) to test its suitability for using in vacuum. As shown in Fig. 3.9, we use two achromatic lenes to focus two laser beams that are propagating in the horizontal direction. The

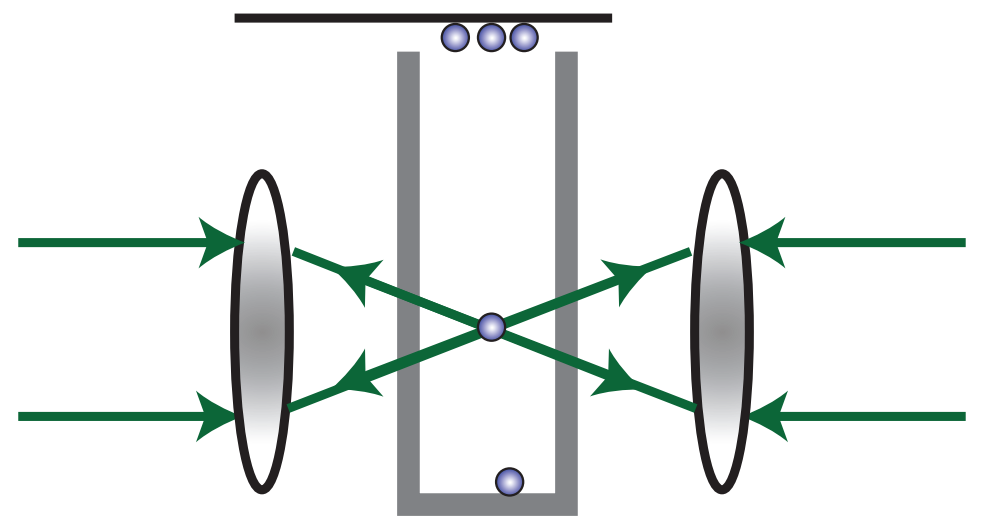

Figure 3.9: A counter-propagating dual-beam optical trap in air. 
focal lengths of the two lenses are $35 \mathrm{~mm}$ and $30 \mathrm{~mm}$, respectively. Because the launched microspheres fall down to the bottom of the glass cell and do not affect the light path, this dual-beam trap is much more stable than the optical levitation trap. We can trap microspheres with diameters of $1.9 \mu \mathrm{m}$, $3.0 \mu \mathrm{m}$, and $4.7 \mu \mathrm{m}$ in air repeatedly. We have also filled the glass cell with water and trapped microspheres in water with this dual-beam trap. This is remarkable because the walls of the glass cell and the water distort the laser beam significantly. Dual-beam optical tweezers are also suitable for trapping particles with high-refractive index in water [66].

The major disadvantage of a counter-propagating dual-beam trap is that the two laser beams must be aligned correctly. Ideally, the two beams should be exactly counter-propagating and focused at the same point. If the two beams are misaligned from each other, the resulting trap will not be a simple harmonic trap. The scattering force from the laser beams is not

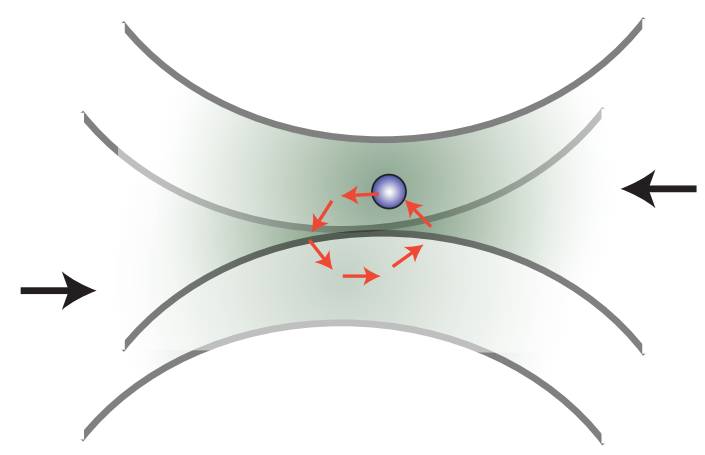

Figure 3.10: A possible motion of a microsphere trapped in a misaligned dualbeam optical tweezer. 
conservative, and can do work on the microsphere under certain conditions [67, 68]. When the two beams are misaligned (Fig. 3.10), the scattering force from the left laser beam will push the microsphere to the right, and the scattering force from the right beam will push the microsphere to the left. This results in a cycled motion. In air (or water), the resulted complex motion can be stable due to the air (or water) damping. We have observed this phenomenon in both air and water. In vacuum, however, the mechanical energy of the microsphere will keep increasing until the microsphere escapes from the trap eventually. This problem can be minimized by aligning the two beams correctly.

As the dual-beam trap in air was much more stable than the optical levitation trap in air, we decided to use the dual-beam trap for trapping microspheres in vacuum. Fig. 3.11 shows a simplified schematic of the first generation of our counter-propagating dual-beam optical trap in vacuum. The two $1064 \mathrm{~nm}$ laser beams for trapping are from a fiber laser whose maximum output power is $10 \mathrm{~W}$. The two laser beams are orthogonally polarized to avoid interference. Their powers are controlled by two $80 \mathrm{MHz}$ acousto-optic modulators (AOM's). The power of beam No. 1 can be changed from $3 \mathrm{~mW}$ to $2 \mathrm{~W}$, and the power of beam No. 2 can be changed from $3 \mathrm{~mW}$ to $3 \mathrm{~W}$. One beam is the +1 order of the output beams from the AOM, and the other beam is the -1 order of the output beams from the AOM. Thus the frequencies of the two laser beams differ by $160 \mathrm{MHz}$. The two laser beams are focused by two identical aspheric lenses whose focal lengths are $3.1 \mathrm{~mm}$. The two lenses are inside a vacuum chamber. 
We collect the scattered light from a trapped microsphere with a lens from the side to monitor the microsphere by a camera. We also use a weak $532 \mathrm{~nm}$ laser beam (about $2 \mathrm{~mW}$ ) and a quadrant detector to monitor the motion of the microsphere with high resolution. The quadrant detector splits the $532 \mathrm{~nm}$ beam into four parts. The difference between the left (top) two parts and the right (bottom) two parts provides the position information of the microsphere in the horizontal (vertical) direction, and the summation of

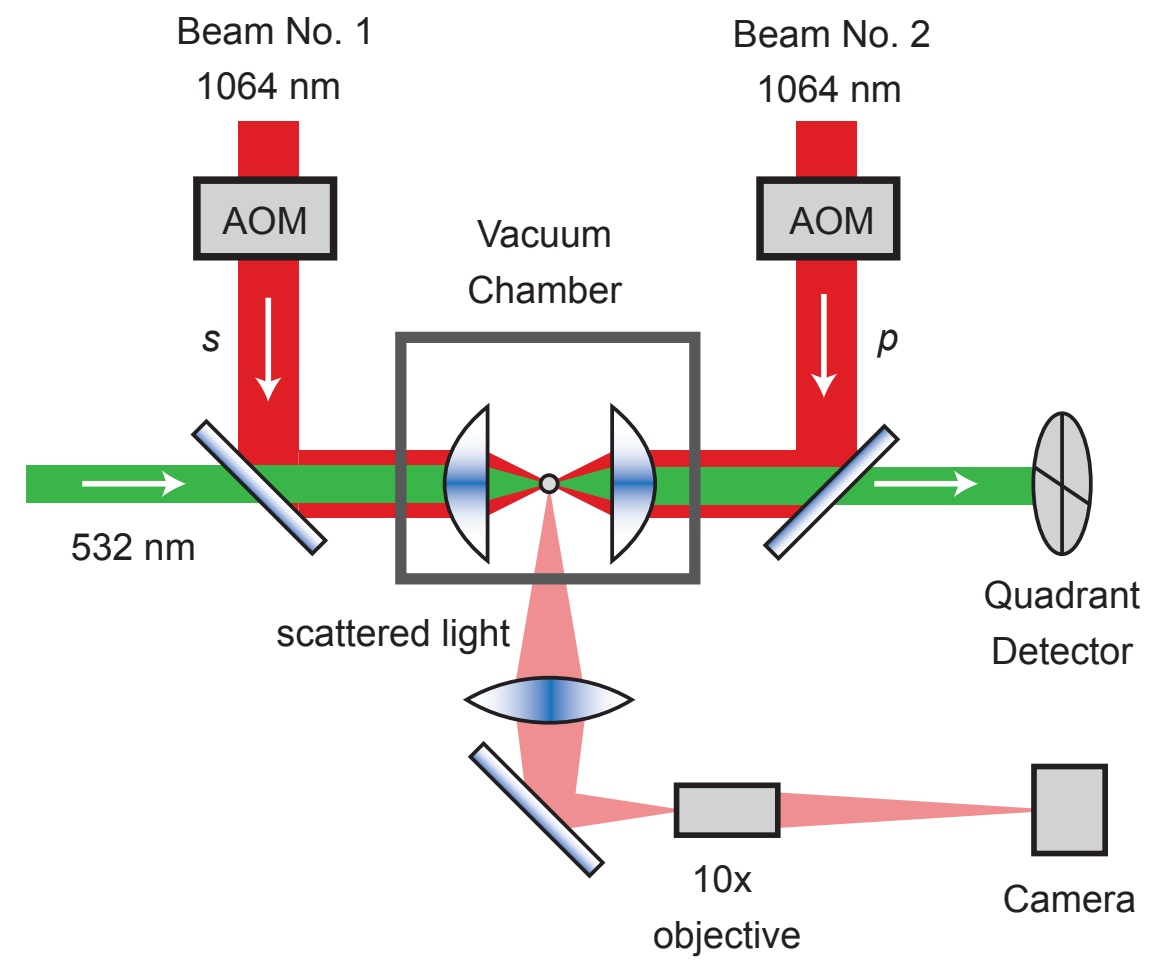

Figure 3.11: A simplified schematic of the first generation of our counterpropagating dual-beam optical trap for trapping microspheres in vacuum, and a detection system to monitor the position of a trapped microsphere. The two $1064 \mathrm{~nm}$ laser beams are orthogonally polarized and have different frequencies to avoid interference. 
the four parts provides the position information of the microsphere along the axial direction [69]. Thus the quadrant detector can monitor the position of a trapped microsphere in 3D.

The setup inside our vacuum chamber is shown in Fig. 3.12. The ultrasonic transducer (Fig. 3.1A) is mounted vertically. The coverslip with microspheres is above the optical trap by about $5 \mathrm{~cm}$. A pair of aspheric lenses (Newport, model: KGA330-B) with focal length of $3.1 \mathrm{~mm}$ are fixed on stainless steel structures with vacuum compatible epoxy. The stainless steel structures are mounted on an aluminum holder by stainless steel screws. The separation between the front surfaces of the two lenses is about $3.5 \mathrm{~mm}$.

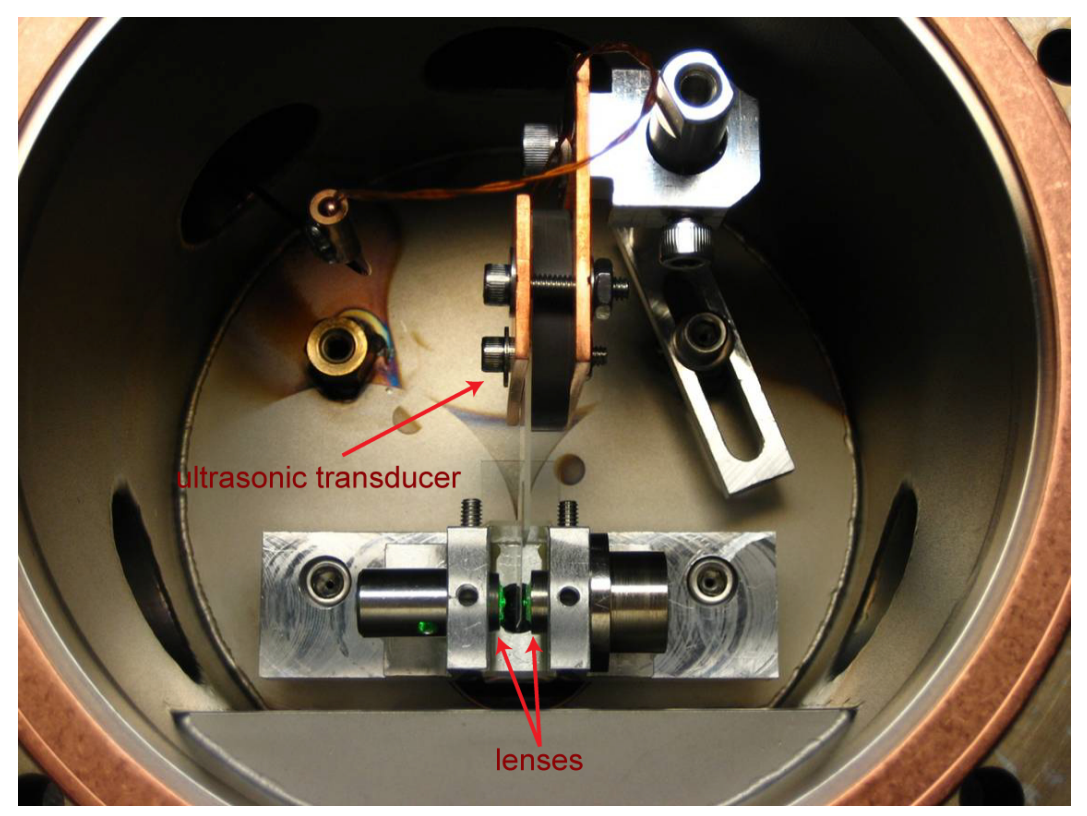

Figure 3.12: Top view of the setup inside the vacuum chamber. A pair of aspheric lenses with focal length of $3.1 \mathrm{~mm}$, and a piezoelectric ultrasonic transducer are inside the vacuum chamber. 
We align the laser beams in three steps. First, we send a parallel $1064 \mathrm{~nm}$ laser beam through the two lenses. One of the two lenses is fixed. We change the position of the other lens to make sure that the output beam after the two lenses is still parallel. Second, we align the directions and divergences of all three laser beams (two $1064 \mathrm{~nm}$ beams and one 532nm beam) to make sure that all beams are on top of each other. Third, we align all laser beams with a pinhole to make sure that the foci of the three beams are at the same point (Fig. 3.13).

The setup for aligning a laser beam and measuring its waist with a pinhole is shown in Fig. 3.13. This setup is good for aligning the trapping beam No. 1 and the detection beam. We move the photodiode and the lens in front of it to the other side of the vacuum chamber for aligning the trapping beam No. 2. The diameter of the pinhole aperture is $1.0 \pm 0.5 \mu \mathrm{m}$. The pinhole is mounted on a 3-axis translation stage. The translation stage is controlled by

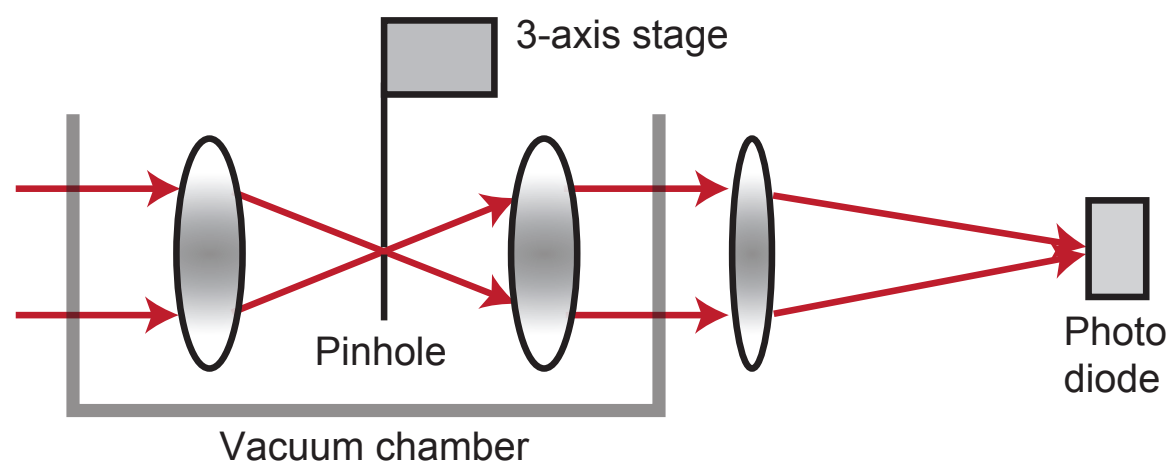

Figure 3.13: Setup for aligning a laser beam and measuring its waist with a pinhole. 
3 manual differential actuators (Thorlabs: DM10) with graduation of $0.5 \mu \mathrm{m}$. In the vertical direction, we also have a piezoelectric actuator to fine tune the pinhole. We first align the pinhole to the focus of the trapping beam No. 1 (by aligning the pinhole to maximize the output power of the beam from the pinhole). The pinhole from National Aperture is easier to align than the high energy pinhole from Newport, because the pinhole from National Aperture is located at the center of a small (about $200 \mu \mathrm{m}$ ) countersink, which can be seen by the naked eye and acts as a guide for alignment. Once the pinhole is aligned to the focus of the trapping beam No. 1, we fine tune the directions and divergences of the detection beam and the trapping beam No. 2 to maximize their output powers from the pinhole. With this method, we can align the focuses of all three laser beams to the same point with an accuracy of about $0.5 \mu \mathrm{m}$.
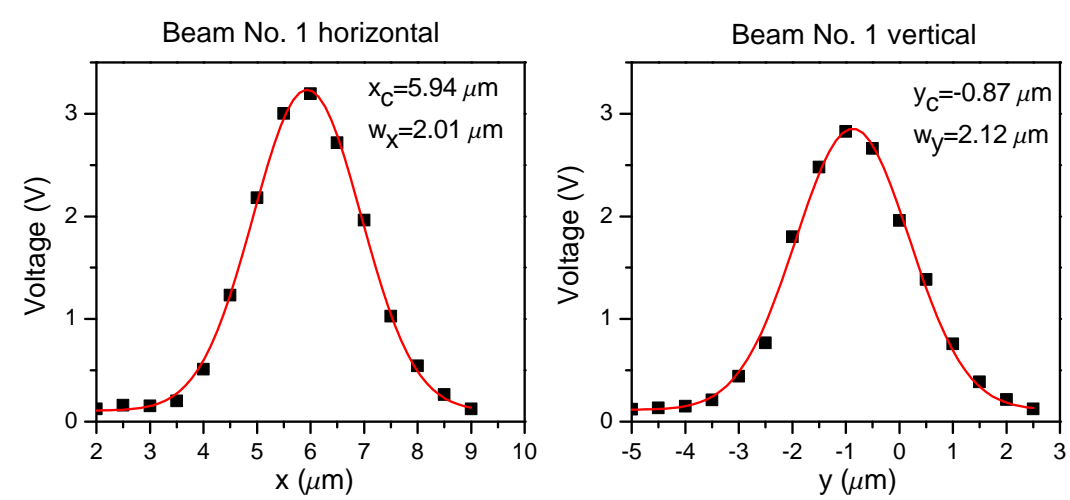

Figure 3.14: An example of measured beam profiles of the beam No. 1 in the horizontal and vertical directions. The red curves are Gaussian functions. 

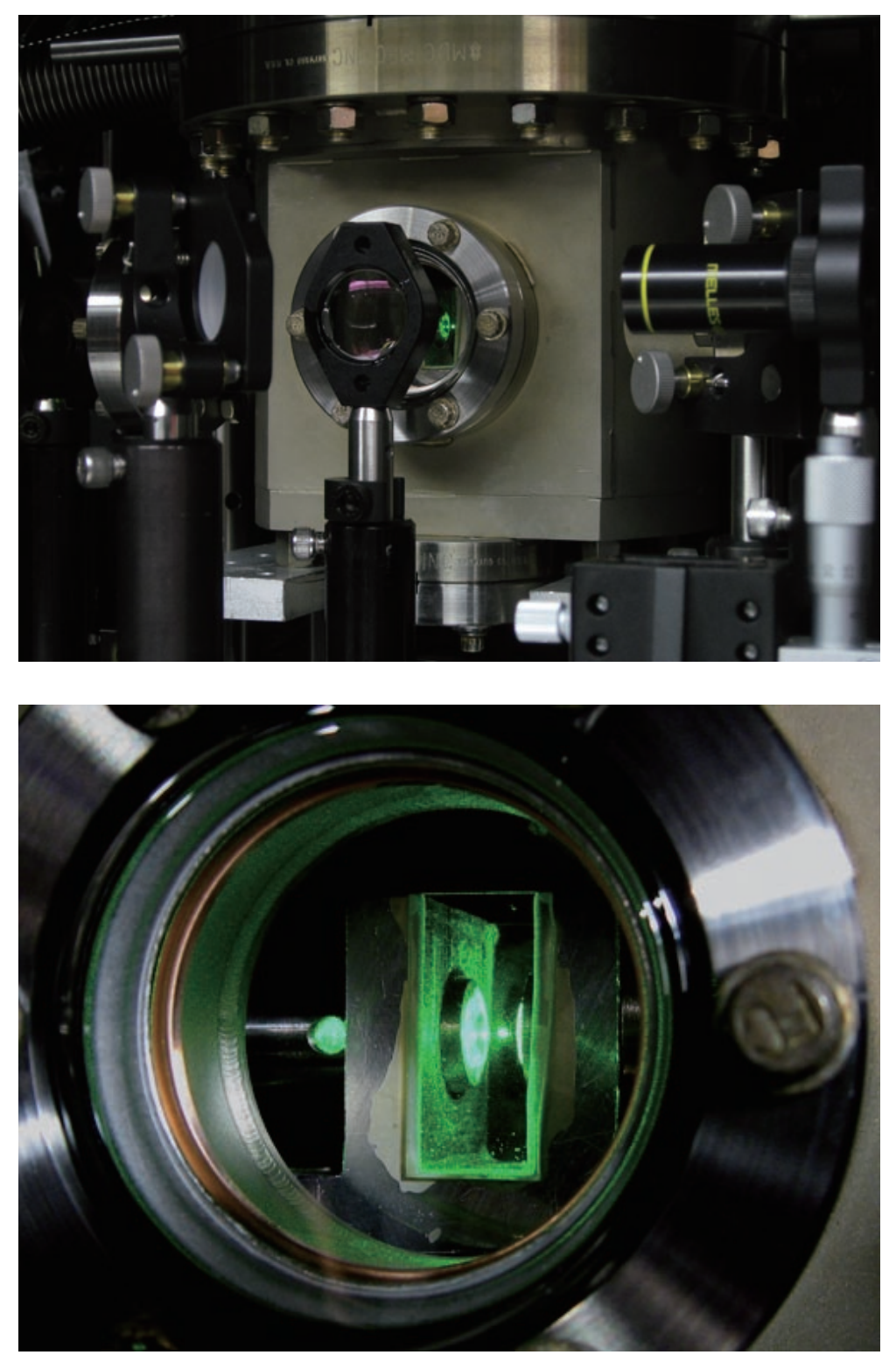

Figure 3.15: A 4.7- $\mu \mathrm{m}$ diameter microsphere trapped inside a vacuum chamber by a counter-propagating dual-beam optical tweezer. The wavelength of the trapping beams is $1064 \mathrm{~nm}$. A weak green (532 nm) laser is used for illumination. 
After alignment, we can scan the pinhole to measure the profiles of the laser beams. Fig. 3.14 shows an example of measured profiles of the beam No. 1 along the horizontal and vertical directions. The measured beam profiles agree with Gaussian functions very well. The measured waists of the beam are $2.01 \mu \mathrm{m}$ in the horizontal direction and $2.12 \mu \mathrm{m}$ in the vertical direction. The real waists of the beam should be smaller than these values because of the finite size of the pinhole aperture.

A 4.7- $\mu \mathrm{m}$ diameter microsphere trapped inside a vacuum chamber by a counter-propagating dual-beam optical tweezer is displayed in Fig. 3.15. The trapping laser is infrared. A weak green laser (about $2 \mathrm{~mW}$ ) is used for illumination. The bright spot near the center of the photos is the trapped microsphere. It usually take us about 10 minutes to trap one microsphere. Once a microsphere is trapped, the system is very stable. We have tested it by trapping a $4.7 \mu \mathrm{m}$ bead in air continuously for 46 hours, during which time
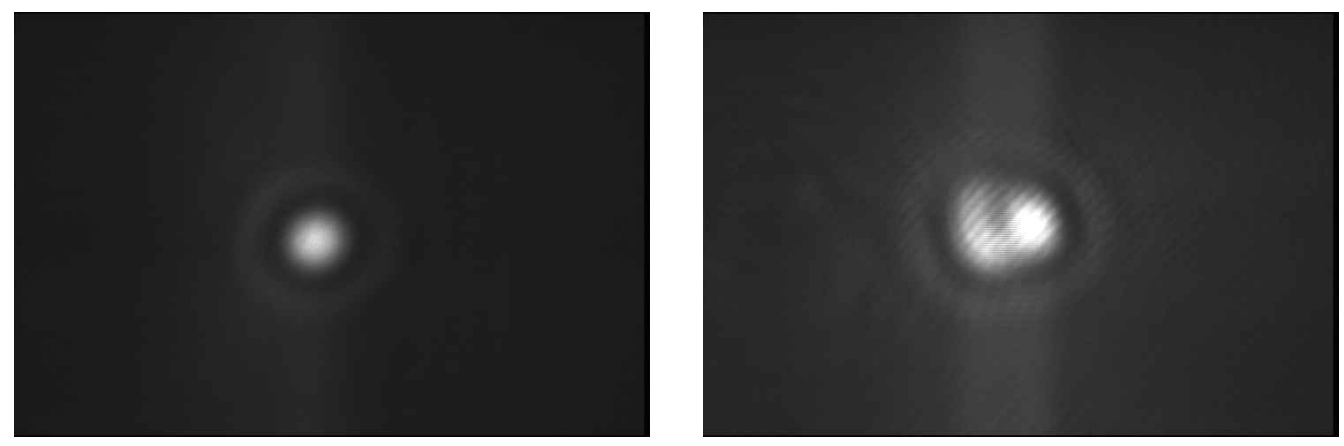

Figure 3.16: Left: A single optically trapped 3.0- $\mu \mathrm{m}$ diameter bead; Right: multiple (2 or maybe 3 ) optically trapped $3.0-\mu \mathrm{m}$ beads. The air pressure is 752 torr. The powers of the two counter-propagating beams are $119 \mathrm{~mW}$ and $100 \mathrm{~mW}$. 
the power of both laser beams was varied between $5 \mathrm{~mW}$ and $2.0 \mathrm{~W}$ to test the stability of the trap. The trap becomes less stable in vacuum. The lowest pressure at which we have trapped a bead without requiring extra stabilization is about $0.1 \mathrm{~Pa}$.

We find that the waists of the two counter-propagating laser beams determine how many microspheres will be trapped by the dual-beam optical tweezer at one time. When the two beams have the same beam waist, we usually trap more than one microsphere (usually two microspheres) at a time. This is because the two counter-propagating laser beams form a double-well potential when their foci are not exactly on top of each other. When the waist of one of the laser beams is larger than the other (for example, $3.0 \mu \mathrm{m}$ for one beam, and $2.0 \mu \mathrm{m}$ for the other beam), we usually trap only one microsphere at a time. The left photo in Fig. 3.16 shows a single $3.0-\mu$ m diameter bead trapped by the dual-beam optical tweezer, and the right photo in Fig. 3.16 shows multiple (2 or maybe 3 ) 3.0- $\mu$ m beads trapped at the same time.

\subsection{Vacuum system}

The science chamber (the vacuum chamber in which we trap microspheres) and most of optic components are mounted on a $3 \times 3$ foot breadboard. The breadboard is supported by elastomers (Newport, Model: NewDamp) on an air-floated optical table to minimize the mechanical vibration. The peak-to-peak vibration amplitude of the lab floor is about $30 \mu \mathrm{m}$ due to seismic vibrations. The peak-to-peak relative vibration amplitude of optical 
elements on an optical table is about $0.1 \mu \mathrm{m}$ (in $10 \mathrm{~s}$ ) when the table is sitting on rigid legs directly. This is reduced to about $10 \mathrm{~nm}$ when the optical table is supported by pneumatic isolators (air-floated). Thus it is essential to use pneumatic isolators to reduce vibrations.

Fig. 3.17 shows a schematic of our vacuum system. The science chamber on the breadboard is connected to a small ion pump on the optical table by a flexible stainless steel bellows to isolate mechanical vibrations. The ion pump is connected to roughing pumps on the lab floor by a bellows. We initially use a sorption pump for rough pumping to avoid mechanical vibrations. The sorption pump reduces air pressure by absorbing molecules within a porous material (molecular sieve) which is cooled by liquid nitrogen. There

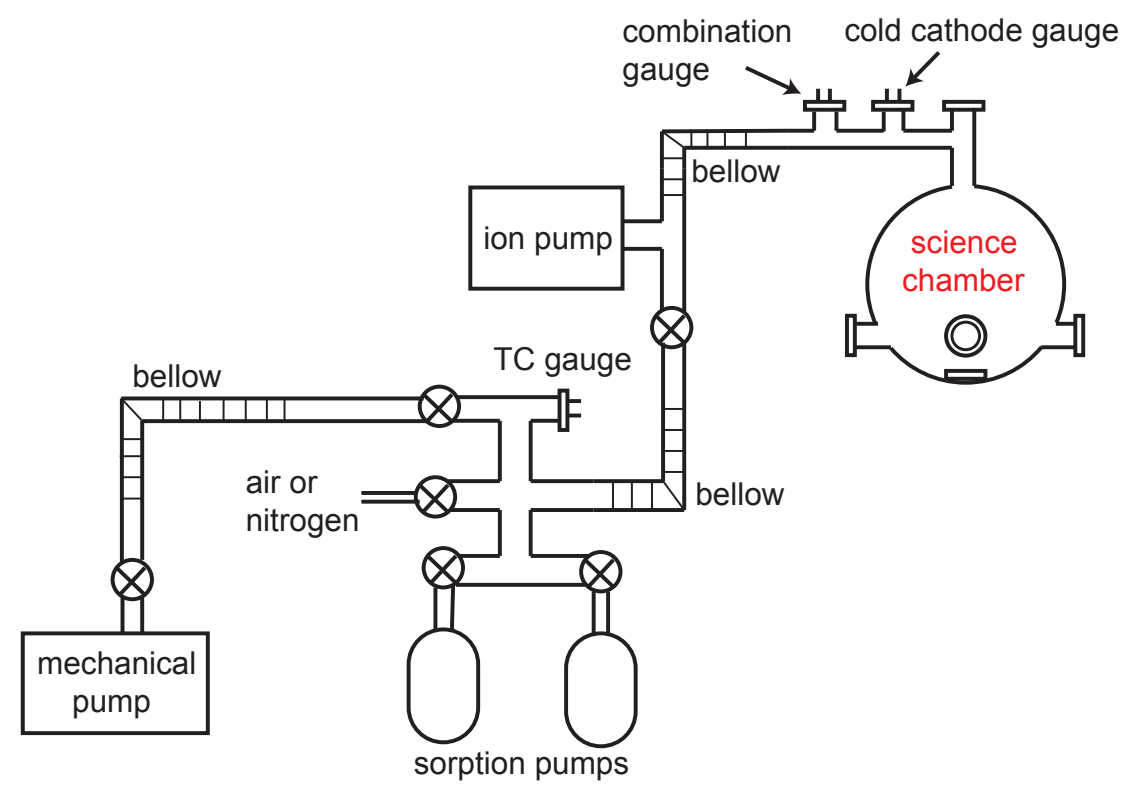

Figure 3.17: A schematic of the vacuum system. 
are no mechanical movable parts in the sorption pump. Thus it has almost no mechanical vibration. The lowest pressure that we can obtain with a single sorption pump is about 3 mtorr $(1$ mtorr $=0.133 \mathrm{~Pa})$. Using two sorption pumps to pump the vacuum chamber sequentially, we can reduce the pressure from 760 torr to about $10^{-5}$ torr in 1 hour.

The sorption pump needs to be heated up to about $200{ }^{\circ} \mathrm{C}$ to drive off absorbed water before using and requires liquid nitrogen to cool it during use, thus it is not convenient to use the sorption pump. We later switched to using a mechanical roughing pump in place of the sorption pump. There are 4 bellows separated by 3 rigid structures between the mechanical pump and the science chamber. They isolates the mechanical vibration sufficiently. There is no observed effect on the motion of a trapped microsphere when we turn on and off the mechanical roughing pump. The mechanical roughing pump can reduce the air pressure from 760 torr (1 atm) to 1 mtorr in a few minutes.

The ion pump can reduce the air pressure from 1 mtorr to about $10^{-6}$ torr in 1 hour without bakeout. At first, the ion pump tended to kick out the trapped microsphere immediately after the pump was turned on. This was caused by momentary electrical arcing (accompanied by a sudden pressure burst) in the ion pump [70]. We later moved the pump further away, and this problem disappeared after we used the ion pump for a few months. With bakeout, one should be able to achieve pressures much lower than $10^{-6}$ torr with the ion pump. The ion pump is not ideal for using with pressures above $10^{-6}$ torr. Since we are able to isolate the mechanical vibration, a turbomolecular pump 
will be a better choice in future.

We have a cold cathode gauge and a combination gauge near the science chamber to measure the air pressure inside the science chamber. The cold cathode gauge (Kurt J. Lesker Company, model: KJLC 943 ) can measure the pressure between $10^{-10}$ torr and $10^{-2}$ torr. The combination gauge (Kurt J. Lesker Company, model: KJLC 910) can measure the pressure between $10^{-5}$ torr and 1500 torr. The combination gauge has a Piezo sensor and a Pirani sensor. The Pirani sensor is gas-type sensitive as it measures pressure based on the thermal conductivity of the gas. The Piezo sensor measures the absolute pressure independent of the gas type. The gauge reads the Piezo sensor when the pressure is above 15 torr, reads the Pirani sensor when the pressure is below 5 torr, and reads both sensors when the pressure is in between. The accuracy of the measurement is $1 \%$ of the reading when the pressure is in the range of $10-1000$ torr, and is about $10 \%$ of the reading for lower pressures. We also have a thermocouple gauge (TC gauge) near the sorption pump to monitor the pressure in the rough pumping region.

There are several manual angle valves between the science chamber and the roughing pumps that can be use to isolate different parts of the system. The vacuum chamber is also connected to a pure nitrogen (or air) gas tube by a gas shut off valve (Kurt J. Lesker Company, model: Nupro BK, SS$4 \mathrm{BK})$. With these valves and vacuum pumps, we can control the pressure in the science chamber from $10^{-6}$ torr to 1500 torr easily. We can achieve much lower pressure with bakeout. The ability to control air pressure allows us to 
study the Brownian motion of a trapped microsphere at different pressures, and cool the motion of a trapped microsphere in vacuum. 


\section{Chapter 4}

\section{Measuring the instantaneous velocity of a Brownian particle in air}

Brownian motion of particles affects many branches of science. The Brownian motion was discovered by R. Brown in 1827 [20], and explained correctly by A. Einstein in 1905 [23]. The trajectories of a Brownian particle are commonly considered to be not differentiable. This is accompanied by the fact that the instantaneous velocity of a Brownian particle has not been measured successfully before. Here we will present our measurement of the instantaneous velocity of a Brownian particle in air.

In this chapter, we will first introduce some theories about the Brownian motion of free particles and trapped microspheres. This will be followed by a description of our home-built detection system that can study the Brownian motion of an optically trapped microsphere in air with an ultrahigh resolution. We will show some measured power spectra of the motion of trapped microspheres at different air pressures. Finally, we will show the results of our measurement of the instantaneous velocity of a Brownian particle in air. Our results provide direct verification of the Maxwell-Boltzmann velocity distribution, and the equipartition theorem for a Brownian particle. 


\subsection{Theories of Brownian motion}

\subsubsection{A free particle}

Einstein's theory of Brownian motion predicts that

$$
\left\langle[\Delta x(t)]^{2}\right\rangle \equiv\left\langle\left(x(t)-(x(0))^{2}\right\rangle=2 D t,\right.
$$

where $\left\langle[\Delta x(t)]^{2}\right\rangle$ is the mean-square displacement (MSD) of a free Brownian particle in one dimension during time $t$, and $D$ is the diffusion constant [23]. The diffusion constant can be calculated by $D=k_{B} T / \gamma$, where $T$ is the temperature, and $\gamma=6 \pi \eta R$ is the Stokes friction coefficient for a sphere with radius $R$. Here $\eta$ is the viscosity of the fluid. The mean velocity measured over an interval of time $t$ is $\bar{v} \equiv \sqrt{\left\langle[\Delta x(t)]^{2}\right\rangle} / t=\sqrt{2 D} / \sqrt{t}$. This diverges as $t$ approaches 0 , and therefore does not represent the real velocity of the particle $[24,25]$.

The equation $\left\langle[\Delta x(t)]^{2}\right\rangle=2 D t$, however, is only valid when $t \gg \tau_{p}$, i.e., in the diffusive regime. Here $\tau_{p}=M / \gamma$ is the momentum relaxation time of a particle with mass $M$. At very short time scales $\left(t \ll \tau_{p}\right)$, the dynamics of a particle is dominated by its inertia, and the motion is ballistic. The dynamics of a Brownian particle with mass $M$ over all time scales can be described by a Langevin equation[71-73]:

$$
M \frac{d^{2} x}{d t^{2}}+\gamma \frac{d x}{d t}=F_{\text {therm }}(t),
$$

where

$$
F_{\text {therm }}(t)=\left(2 k_{B} T \gamma\right)^{1 / 2} \zeta(t)
$$


is the Brownian stochastic force. $\zeta(t)$ is a normalized white-noise process. Hence for all $t$ and $t^{\prime}$,

$$
\langle\zeta(t)\rangle=0 \text {, and }\left\langle\zeta(t) \zeta\left(t^{\prime}\right)\right\rangle=\delta\left(t-t^{\prime}\right) \text {. }
$$

Let $v(t)=d x / d t$, and divide the Eq. 4.2 by $M$, we obtain

$$
\frac{d v(t)}{d t}=-\Gamma_{0} v(t)+A(t)
$$

where $\Gamma_{0}=\gamma / M=1 / \tau_{p}$ is the damping coefficient and $A(t)=F_{\text {therm }}(t) / M$ is the fluctuating acceleration. The velocity and position of the particle at time $t=0$ are $v(0)=v_{0}$ and $x(0)=x_{0}$, respectively. Then its velocity at time $t$ is [73]:

$$
v(t)=v_{0} e^{-\Gamma_{0} t}+e^{-\Gamma_{0} t} \int_{0}^{t} e^{\Gamma_{0} s} A(s) d s .
$$

Taking the mean over an ensemble of particles, which have started at $t=0$ with the same velocity $v_{0}$, and using Eq. 4.4 we get:

$$
\begin{gathered}
\langle v(t)\rangle_{v_{0}}=v_{0} e^{-\Gamma_{0} t}, \\
\langle v(t) v(0)\rangle_{v_{0}}=v_{0}^{2} e^{-\Gamma_{0} t} .
\end{gathered}
$$

Taking a second average over $v_{0}$ and using the energy equipartition theorem $\left\langle M v_{0}^{2} / 2\right\rangle=k_{B} T / 2$, we obtain the velocity autocorrelation function:

$$
\langle v(t) v(0)\rangle=\frac{k_{B} T}{M} e^{-\Gamma_{0} t} .
$$

By integrating Eq. 4.6 again, we get the position of the particle [73]:

$$
x(t)=x_{0}+\frac{v_{0}}{\Gamma_{0}}\left(1-e^{-\Gamma_{0} t}\right)+\int_{0}^{t} e^{-\Gamma_{0} s_{1}} d s_{1} \int_{0}^{s_{1}} e^{\Gamma_{0} s_{2}} A\left(s_{2}\right) d s_{2} .
$$


Taking the average, we get:

$$
\langle\Delta x(t)\rangle_{x_{0}}=\langle x(t)-x(0)\rangle_{x_{0}}=\frac{v_{0}}{\Gamma_{0}}\left(1-e^{-\Gamma_{0} t}\right) .
$$

The MSD for a Brownian particle at thermal equilibrium with the air is [73]:

$$
\left\langle[\Delta x(t)]^{2}\right\rangle=\frac{2 k_{B} T}{M \Gamma_{0}^{2}}\left(\Gamma_{0} t-1+e^{-\Gamma_{0} t}\right) .
$$

At long time scales, the MSD is the same as the prediction of Einstein's theory:

$$
\left\langle[\Delta x(t)]^{2}\right\rangle=2 D t \quad \text { for } \quad t \gg \tau_{p}
$$

At very short time scales, the MSD is

$$
\left\langle[\Delta x(t)]^{2}\right\rangle=\frac{k_{B} T}{M} t^{2} \quad \text { for } \quad t \ll \tau_{p} .
$$

Although the above equations are derived for an ensemble of identical particles, the ergodic theorem dictates that they are also valid for measurements of a single particle taken over a long time.

At very short time scales, the motion is ballistic and its instantaneous velocity can be measured as $v=\Delta x(t) / t$, when $t \ll \tau_{p}$ [73]. The ballistic Brownian motion is different from a simple ballistic motion. For a simple ballistic motion with velocity $u$, we have $\Delta x(t)=u t$ and $[\Delta x(t)]^{2}=u^{2} t^{2}$. The velocity $u$ can be any value and usually has no relation with the temperature of the environment.

The 1D Maxwell-Boltzmann distribution of the velocity of a particle in thermal equilibrium is

$$
f_{v}\left(v_{i}\right)=\sqrt{\frac{M}{2 \pi k_{B} T}} \exp \left(-\frac{M v_{i}^{2}}{2 k_{B} T}\right),
$$

where $v_{i}$ is the velocity of the particle along any direction $i$. 


\subsubsection{A trapped microsphere}

For small displacements, the effect of optical tweezers on the microsphere's motion can be approximated by that of a harmonic potential. The equation of the Brownian motion of a microsphere in a harmonic trap is:

$$
\frac{d^{2} x}{d t^{2}}+\Gamma_{0} \frac{d x}{d t}+\Omega^{2} x=\Lambda \zeta(t)
$$

where $\Omega=\sqrt{\kappa / m}$ is the natural angular frequency of the trapped microsphere when there is no damping, and $\Lambda=\left(2 k_{B} T \Gamma_{0} / M\right)^{1 / 2}$. The cyclic frequency of the damped oscillator is $\omega_{1}=\sqrt{\Omega^{2}-\Gamma_{0}^{2} / 4}$. The system is underdamped when $\omega_{1}$ is real $(\Omega>\Gamma / 2)$, critically damped when $\omega_{1}=0$, and overdamped when $\omega_{1}$ is imaginary $(\Omega<\Gamma / 2)$.

\subsubsection{Displacement and velocity}

The MSD of a Brownian particle in an underdamped harmonic trap in air can be obtained by solving Eq. 4.16 [74]:

$$
\left\langle[\Delta x(t)]^{2}\right\rangle=\frac{2 k_{B} T}{M \Omega^{2}}\left[1-e^{-t / 2 \tau_{p}}\left(\cos \omega_{1} t+\frac{\sin \omega_{1} t}{2 \omega_{1} \tau_{p}}\right)\right] .
$$

The position autocorrelation function is related to the MSD by:

$$
\left\langle[\Delta x(t)]^{2}\right\rangle=2\left\langle x^{2}\right\rangle-2\langle x(t) x(0)\rangle
$$

where $\left\langle x^{2}\right\rangle=k_{B} T /\left(M \Omega^{2}\right)$. The rms amplitude is $x_{r m s}=\sqrt{k_{B} T /\left(M \Omega^{2}\right)}$. The normalized position autocorrelation function (PACF) of the particle is [74]:

$$
\frac{\langle x(t) x(0)\rangle}{\left\langle x^{2}\right\rangle}=e^{-t / 2 \tau_{p}}\left(\cos \omega_{1} t+\frac{\sin \omega_{1} t}{2 \omega_{1} \tau_{p}}\right) .
$$


The normalized velocity autocorrelation function (VACF) of the particle is [74]:

$$
\frac{\langle v(t) v(0)\rangle}{\left\langle v^{2}\right\rangle}=e^{-t / 2 \tau_{p}}\left(\cos \omega_{1} t-\frac{\sin \omega_{1} t}{2 \omega_{1} \tau_{p}}\right) .
$$

Both the position autocorrelation function and the velocity autocorrelation function oscillate for an underdamped system.

For an overdamped system, $\omega_{1}$ is imaginary. We can rewrite the above

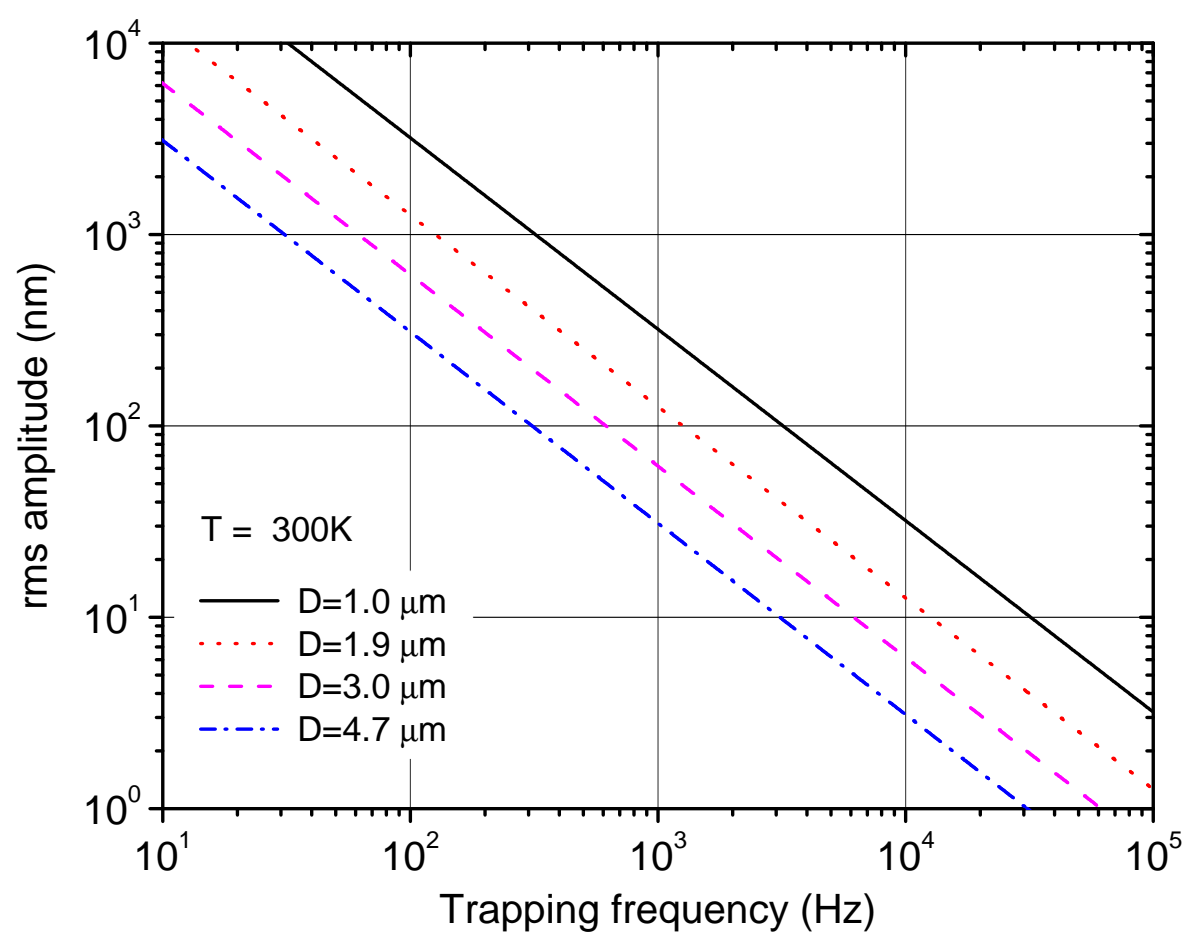

Figure 4.1: The rms amplitude $\left(x_{r m s}\right)$ of a microsphere in a harmonic trap with trapping frequency $\Omega /(2 \pi)$ at thermal equilibrium with the environment. 
formula for the normalized position and velocity autocorrelations as [75]:

$$
\begin{gathered}
\frac{\langle x(t) x(0)\rangle}{\left\langle x^{2}\right\rangle}=\frac{1}{2\left|\omega_{1}\right| \tau_{+}} e^{-t / \tau_{-}}-\frac{1}{2\left|\omega_{1}\right| \tau_{-}} e^{-t / \tau_{+}}, \\
\frac{\langle v(t) v(0)\rangle}{\left\langle v^{2}\right\rangle}=-\frac{1}{2\left|\omega_{1}\right| \tau_{-}} e^{-t / \tau_{-}}+\frac{1}{2\left|\omega_{1}\right| \tau_{+}} e^{-t / \tau_{+}},
\end{gathered}
$$

where

$$
\tau_{ \pm}=\frac{2 \tau_{p}}{1 \pm 2 \tau_{p}\left|\omega_{1}\right|}
$$

Thus the correlations in an overdamped system decrease as a double-exponential with characteristic times $\tau_{ \pm}$. A numerical simulation of the dynamics of harmonic oscillator with different damping can be found in Ref. [75].

\subsubsection{Power spectrum analysis}

Similar to the optical spectrum of an atom, the power spectrum of the Brownian motion of a trapped microsphere contains a lot of information about the system. The power spectral density (PSD) of a variable is the squared modulus of its Fourier transform [74-76].

The Fourier transformations of $x(t)$ and $\zeta(t)$ are:

$$
\begin{aligned}
& \widetilde{x_{k}}=\int_{-T_{r e c} / 2}^{T_{r e c} / 2} e^{i \omega_{k} t} x(t) d t, \\
& \widetilde{\zeta_{k}}=\int_{-T_{r e c} / 2}^{T_{r e c} / 2} e^{i \omega_{k} t} \zeta(t) d t,
\end{aligned}
$$

where $\omega_{k}=2 \pi k / T_{r e c}, k$ is an integer, and $T_{r e c}$ is the time duration of the recorded positions of a trapped microsphere. 
Fourier transforming Eq. 4.16 gives

$$
-\omega_{k}^{2} \widetilde{x_{k}}-i \omega_{k} \Gamma_{0} \widetilde{x_{k}}+\Omega^{2} \widetilde{x_{k}}=\Lambda \widetilde{\zeta_{k}}
$$

Thus

$$
\widetilde{x_{k}}=\frac{\Lambda \widetilde{\zeta_{k}}}{\Omega^{2}-\omega_{k}^{2}-i \omega_{k} \Gamma_{0}} .
$$

From Eq. 4.4 we have $<\widetilde{x_{k}}>=0$ and $<\widetilde{\zeta_{k}} \widetilde{\zeta}_{l}>=T_{r e c} \delta_{k l}$. The PSD of an

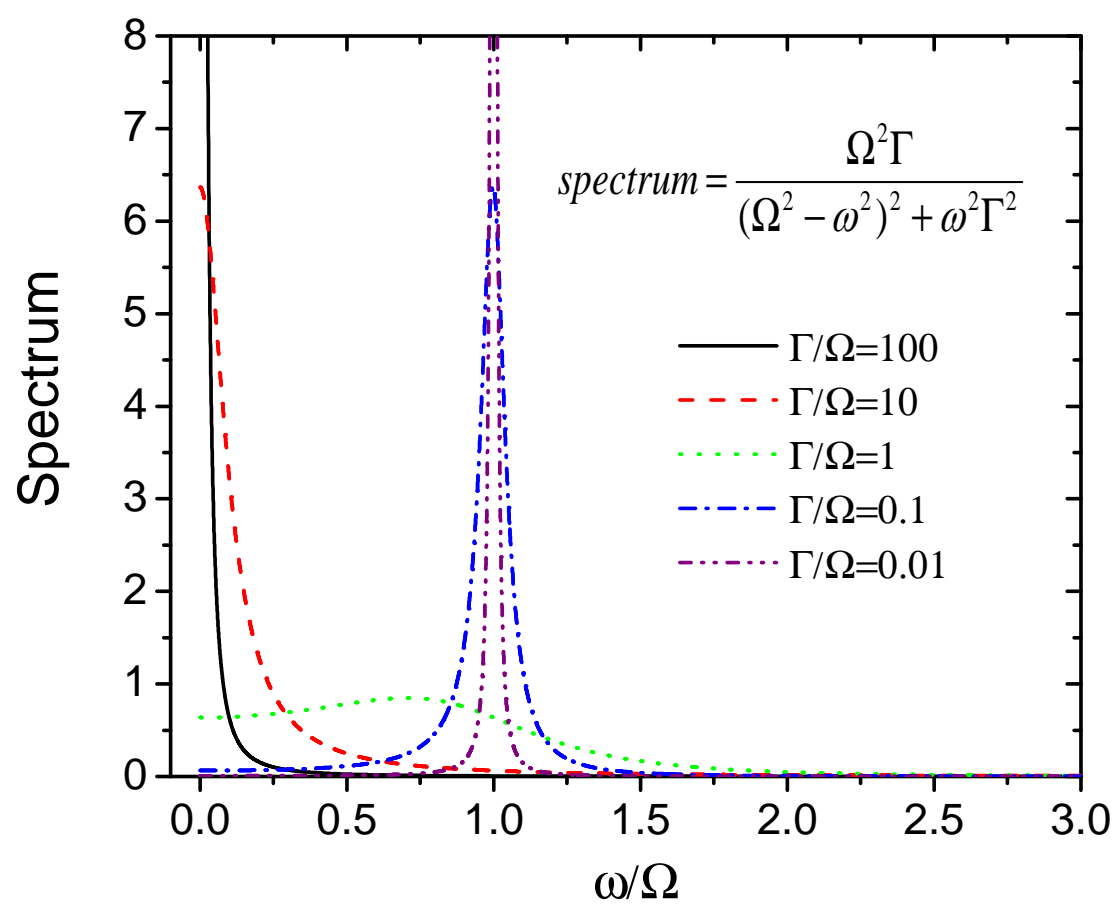

Figure 4.2: Calculated spectra $\left(f_{S}(\omega)\right)$ for systems with different damping. The plot is displayed in linear scales. 
experimentally recorded $x(t)$ is

$$
S_{k}^{r e c} \equiv\left|\widetilde{x_{k}}\right|^{2} / T_{r e c}=\frac{\left|\widetilde{\zeta_{k}}\right|^{2}}{T_{r e c}} \frac{2 k_{B} T}{M \Omega^{2}} \frac{\Omega^{2} \Gamma_{0}}{\left(\Omega^{2}-\omega_{k}^{2}\right)^{2}+\omega_{k}^{2} \Gamma_{0}^{2}} .
$$

The expected values of the PSD is

$$
S(\omega) \equiv<S_{k}^{r e c}>=\frac{2 k_{B} T}{M \Omega^{2}} \frac{\Omega^{2} \Gamma_{0}}{\left(\Omega^{2}-\omega^{2}\right)^{2}+\omega^{2} \Gamma_{0}^{2}} .
$$

The real measured PSD of a recorded $x(t)$ is $S_{k}^{r e c}$. It has a $\left|\widetilde{\zeta}_{k}\right|^{2} / T_{\text {rec }}$ term, which is a random number. Thus an experimental PSD will appear

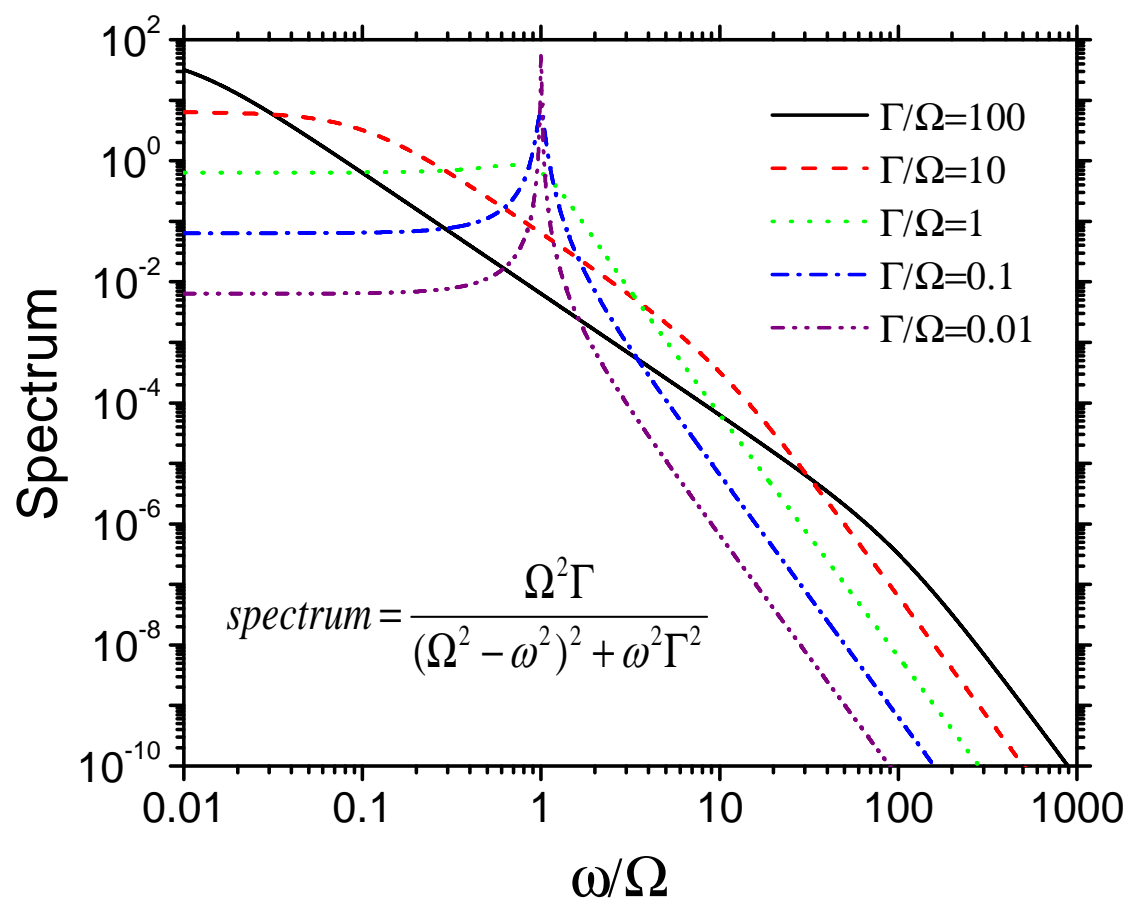

Figure 4.3: Calculated spectra $\left(f_{S}(\omega)\right)$ for systems with different damping. The plot is displayed in log-log scales. 
noisy. Averaging many measured $S_{k}^{r e c}$ will result in a spectrum close to the expected spectrum $S(\omega)$. Another way to reduce the noise in a measured spectrum is "blocking" [76]. Here, a "block" of consecutive data points $\left(\omega_{k_{1}}, S_{k_{1}}^{r e c}\right) \ldots$ $\left(\omega_{k_{2}}, S_{k_{2}}^{r e c}\right)$ is replaced with a single new "data point" $\left(\overline{\omega_{k}}, \overline{S_{k}^{r e c}}\right)$ which are the block averages.

Let us define a new function to show the shape of the spectrum

$$
f_{S}(\omega) \equiv \frac{\Omega^{2} \Gamma_{0}}{\left(\Omega^{2}-\omega^{2}\right)^{2}+\omega^{2} \Gamma_{0}^{2}}
$$

We have

$$
\int_{0}^{\infty} f_{S}(\omega) d \omega=\frac{\pi}{2}
$$

thus

$$
\int_{0}^{\infty} S(\omega) d \omega=\pi \frac{k_{B} T}{M \Omega^{2}}=\pi<x^{2}>.
$$

So the integral of the PSD is proportional to the square of the rms amplitude. Some calculated spectra $f_{S}(\omega)$ for systems with different damping are shown in Fig. 4.2 (in linear scales) and Fig. 4.3 (in log-log scales). As dramatically displayed in the linear scale plot (Fig. 4.2), the spectra have large values at low frequencies for an overdamped system, and have large values at the resonant frequency for an underdamped systems.

\subsection{A fast detection system}

In order to measure the instantaneous velocity of the Brownian motion of a trapped microsphere in air, we implemented an ultrahigh resolution detection system. 
Our first generation of the dual-beam optical trap in vacuum (Fig. 3.11) used a $10 \mathrm{~W}$ fiber laser at $1064 \mathrm{~nm}$ for trapping, and a weak $532 \mathrm{~nm}$ laser for detection. We used a quadrant detector to monitor the position of a trapped microsphere in 3D. That system was not ideal for our experiment. The peak-to-peak intensity noise (in the range of $10 \mathrm{kHz}-1 \mathrm{MHz}$ ) of the fiber laser is about $4 \%$, which is very big. The $532 \mathrm{~nm}$ laser has much less noise than the fiber laser. However, the quadrant detector has significant electronic noise and its bandwidth is only about $1 \mathrm{MHz}$.

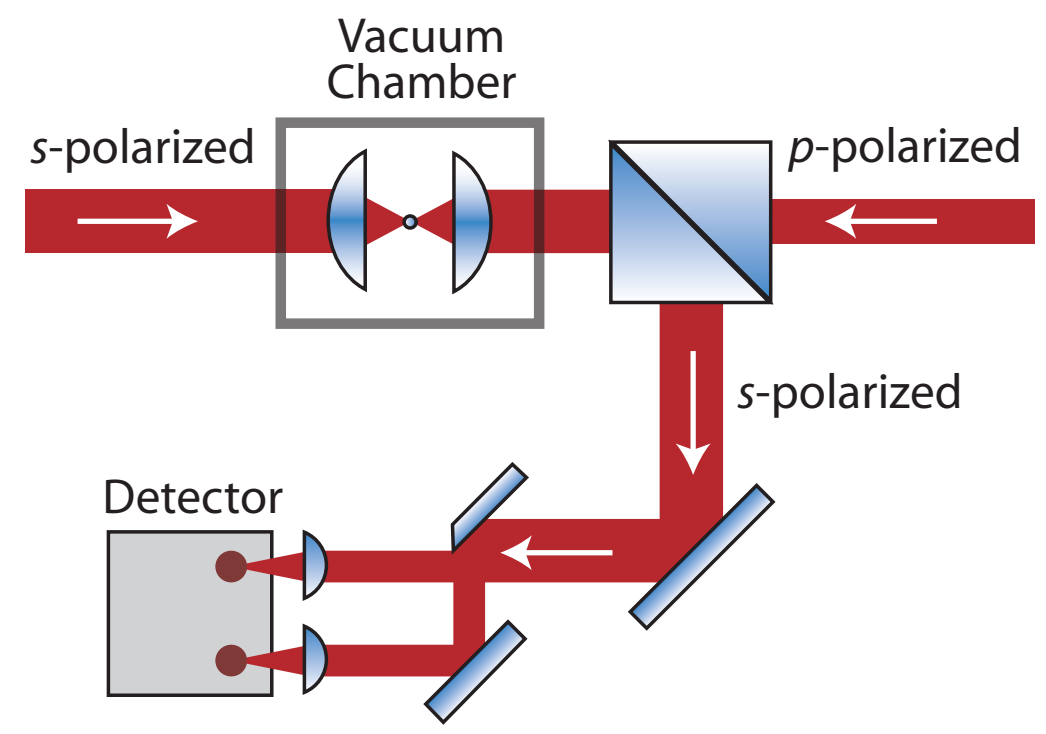

Figure 4.4: This simplified schematic shows our counter-propagating dualbeam optical tweezers, and a novel detection system that has $75 \mathrm{MHz}$ bandwidth and low noise. The $s$-polarized beam is reflected by a polarizing beam splitter cube after it passes through a trapped bead inside a vacuum chamber. Then, for detection, it is split by a mirror with a sharp edge. The $p$-polarized beam passes through the cube. 
In the second-generation experiment with the dual-beam optical trap in vacuum, we use an ultra-stable NPRO laser (Model: 126-1063-700, Lightwave Electronics (now JDSU)) to replace the fiber laser. Its rms intensity noise is $<0.05 \%$ over the range from $10 \mathrm{~Hz}$ to $2 \mathrm{MHz}$, and is shot noise limited above $10 \mathrm{MHz}$. It is a single frequency laser with a linewidth $<5 \mathrm{kHz} / \mathrm{ms}$ and a coherence length longer than $1000 \mathrm{~m}$. A detailed characterization of this type of laser can be found in Ref. [77]. These values are much better than those of the $532 \mathrm{~nm}$ laser. So we use this laser for both trapping and detection. This is achieved by using a polarizing beam splitter cube to reflect one of the trapping beams for detection (Fig. 4.4).

Our lab has previously developed a fast position-sensitive laser beam detector for studying the Brownian motion of particles in water at fast time scales [28]. The previous detector used a fiber-optic bundle that spatially splits the incident beam, and a fast balanced photodetector to measure the difference between the two halves of the beam. Here we simplify the detection system by using a mirror with a sharp edge (BBD05-E03, Thorlabs) to replace the fiber-optic bundle for splitting the beam. The sharp edge of a mirror is much smoother than the boundary between the two halves of a fiber-optic bundle. Thus a mirror with a sharp edge is not only much simpler, but also has less noise than a fiber bundle for splitting the laser beam [28].

We use a balanced detector (PDB120C, photodiode diameter $0.3 \mathrm{~mm}$, Thorlabs) with a bandwidth of $75 \mathrm{MHz}$ for detection. The detector is sensitive to light with wavelengths in the range of $800-1700 \mathrm{~nm}$. It has a high 


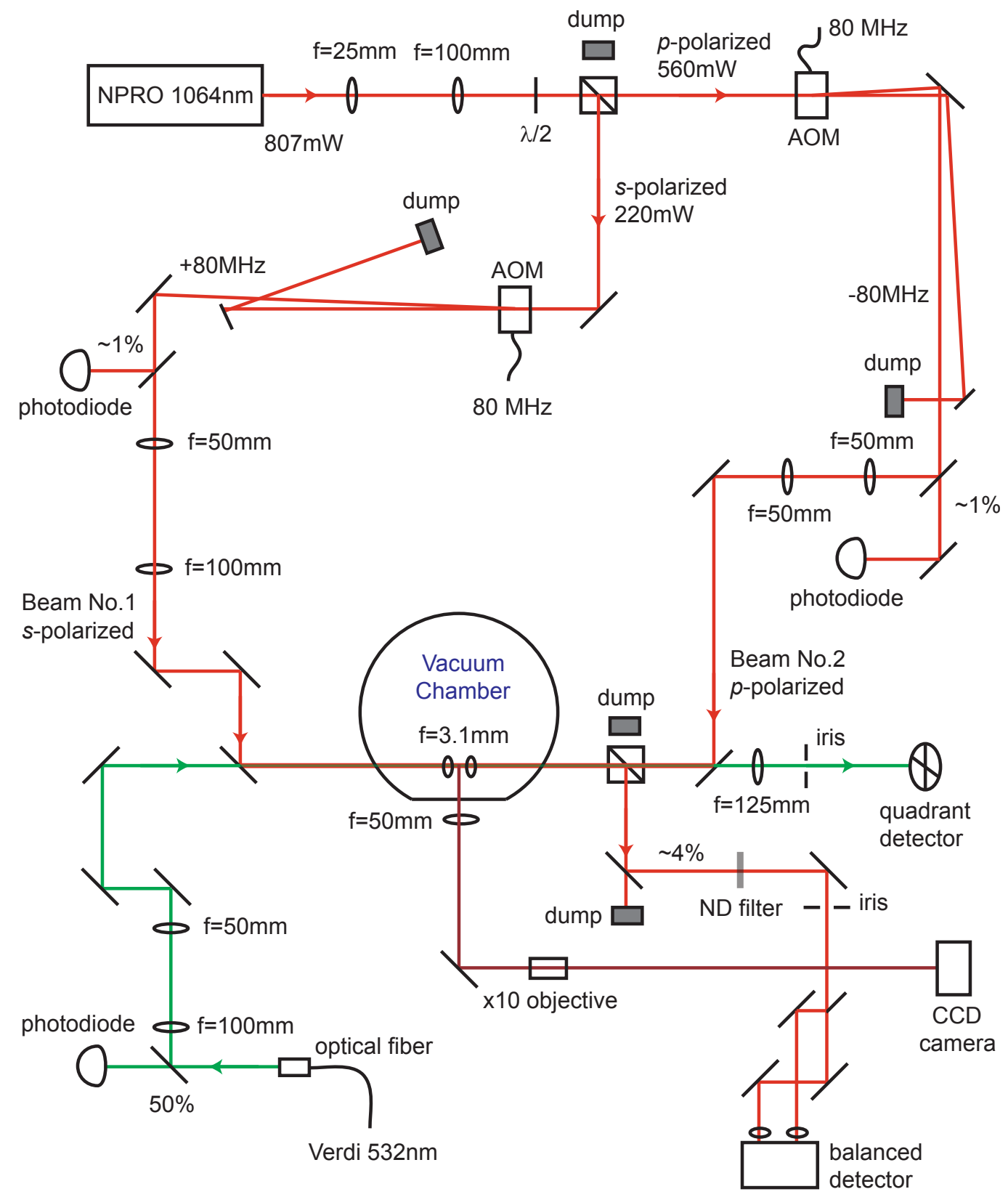

Figure 4.5: The optical circuit of the counter-propagating dual-beam optical trap and the detection system. 
transimpedance gain of $1.8 \times 10^{5} \mathrm{~V} / \mathrm{A}$. The detector measures the difference between the two halves of the beam, which is proportional to the particle excursion. The intensity noise of the laser is contained in both halves and is thus canceled in the measurement. This detection system enables us to monitor the real-time position of a trapped microsphere with Ångstrom spatial resolution and microsecond temporal resolution.

The major optical circuit of our second generation dual-beam trap and the detection system is shown in Fig. 4.5. The powers of the two trapping beams and the probe beam are controlled by AOM's. We use photodiodes to monitor the powers of the beams. With measured powers, we use analog proportional-integral-derivative (PID) circuits to stabilize the laser powers. This eliminates the drifts of the laser powers. The size of the beam No. 1 before entering the vacuum chamber is twice of the size of the beam No. 2 . This ensures that the optical tweezer traps only one microsphere at a time. The green laser at $532 \mathrm{~nm}$ is helpful for aligning the laser beams and monitoring microspheres before they are trapped. Once a microsphere is trapped, we can see it on a VCR monitor connected to the CCD camera. The quadrant detector and the balanced detector give high resolution signals of the motion of a trapped microsphere. For studying the Brownian motion, we turned off the $532 \mathrm{~nm}$ laser, and use only the balanced detector to monitor the position of a trapped microsphere with ultrahigh precision. 


\subsection{Measured power spectra}

Figure 4.6 shows a waveform and a spectrum of a 3.0- $\mu$ m diameter bead trapped by a dual-beam optical tweezer at 749 torr. The powers of the two trapping beams are $10.7 \mathrm{~mW}$ and $14.1 \mathrm{~mW}$. The data were taken by a DAQ card (PCI 6133, National Instruments) controlled by a Labview program. The waveform and spectrum shown here were saved from the Labview program directly. The sampling rate was $2 \mathrm{MHz}$, and the time duration was 2 s. So the waveform has 4 million data points. As we can see, the waveform appears as a white noise and has very little information.

The power spectrum of the waveform, on the other, is much more instructive. The power spectrum in Fig. 4.6 is in log-log scales. Comparing it with the spectra in Fig. 4.3, we can find that its trapping frequency $(\Omega /(2 \pi))$ is about $3 \mathrm{kHz}$, and the linewidth $\left(\Gamma_{0} /(2 \pi)\right)$ is close to the trapping frequency. At high frequencies near $1 \mathrm{MHz}$, the shape of the measured spectrum is very different from the shape of the expected spectrum as shown in Fig. 4.3. This is because at frequencies near $1 \mathrm{MHz}$, the real signal due to the Brownian motion of the trapped microsphere is smaller than the spectrum of noise (laser noise, electronic noises of the detector and the DAQ card). So the displayed spectrum near $1 \mathrm{MHz}$ is the spectrum of noise rather than the real signal.

Figure 4.7 shows a waveform and spectrum of a $3.0-\mu \mathrm{m}$ diameter bead trapped by a dual-beam optical tweezer at 20.6 torr. Other conditions are the same as those in Fig. 4.6. Because of less damping, a peak appears at the trapping frequency. The spectrum at high frequencies (around $100 \mathrm{kHz}$ ) is 
also smaller than those in Fig. 4.6, in agreement of the theoretical predications (Fig. 4.3).

Sometimes we can trap more than one microsphere at the same time (Fig. 3.16). Fig. 4.8 shows a waveform and spectrum of multiple (2 or 3 ) microspheres trapped at 752 torr. The powers of the two laser beams are 119 $\mathrm{mW}$ and $100 \mathrm{~mW}$. The spectrum has new peaks at frequencies around $300 \mathrm{kHz}$.
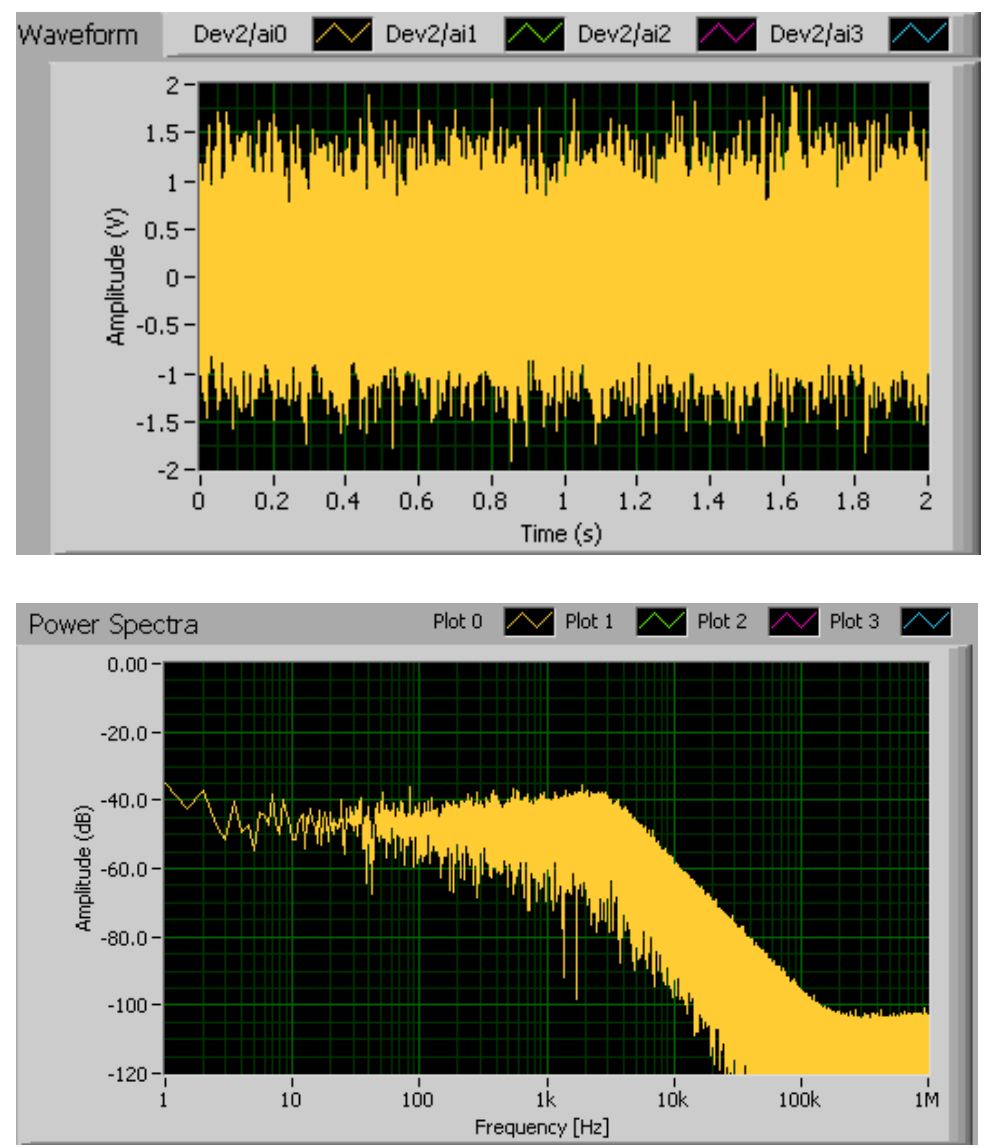

Figure 4.6: A waveform (Top) and a spectrum (Bottom) of a 3.0- $\mu \mathrm{m}$ bead trapped at 749 torr. The powers of the two trapping beams are $10.7 \mathrm{~mW}$ and $14.1 \mathrm{~mW}$. 
These peaks are not presented when there is only one microsphere. They are due to the relative motion between the trapped microspheres. The frequencies of relative motion are much larger than the frequency of the center-of-mass motion (about $1.5 \mathrm{kHz}$ ) in this example.

The spectra of the relative motion between multiple microspheres depend strongly on the details of the optical trap, such as the alignment of the
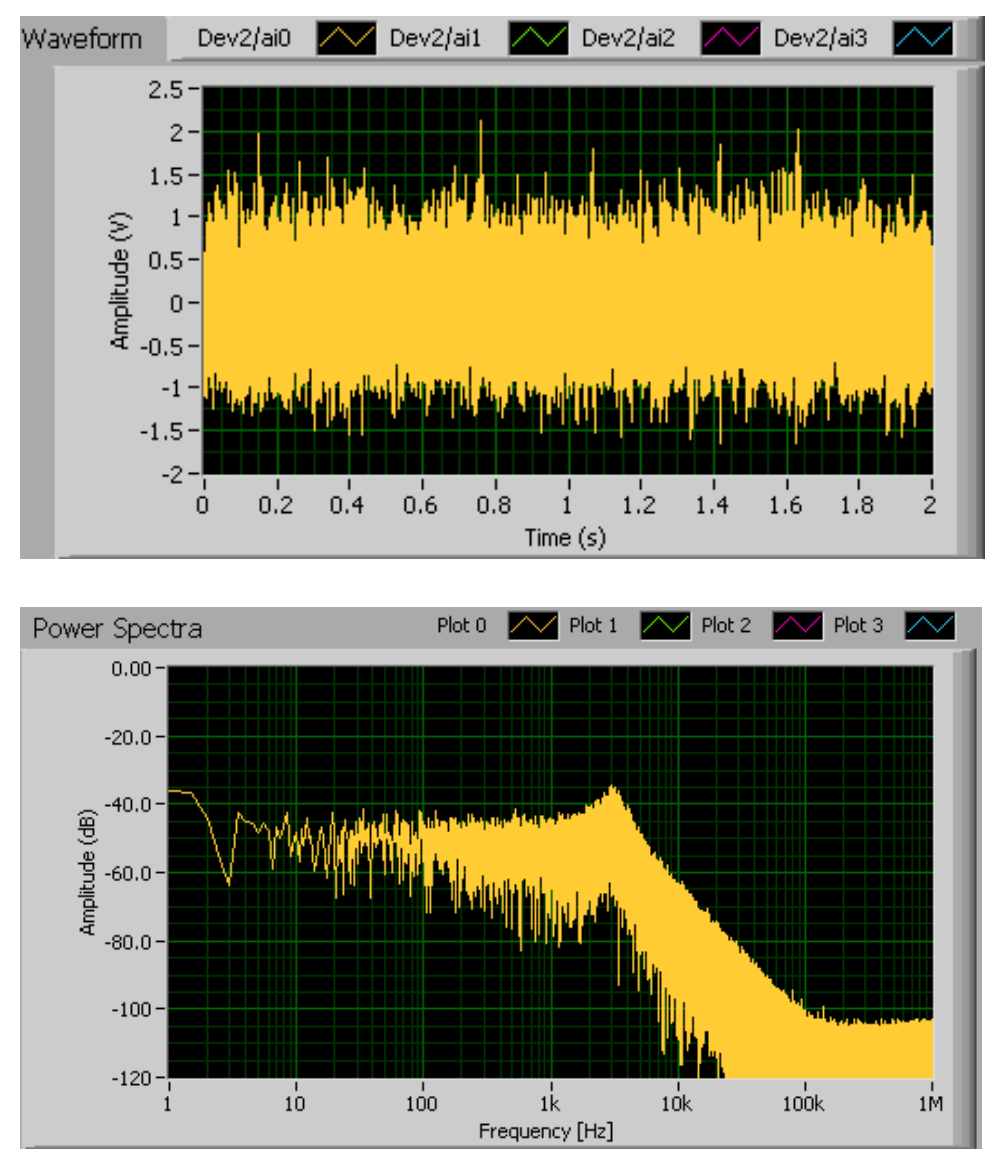

Figure 4.7: A waveform (Top) and a spectrum (Bottom) of a 3.0- $\mu \mathrm{m}$ bead trapped at 20.6 torr. The powers of the two trapping beams are $10.7 \mathrm{~mW}$ and $14.1 \mathrm{~mW}$. 
laser beams and the size of the microspheres. Fig. 4.9 shows the spectra of two 4.7- $\mu \mathrm{m}$ beads trapped at 9.78 torr with different laser power. The frequency of the highest peak is about $70 \mathrm{kHz}$ when the powers of the two trapping beams are $0.7 \mathrm{~W}$ and $1.0 \mathrm{~W}$, and is about $150 \mathrm{kHz}$ when the powers of the two trapping beams are $2.0 \mathrm{~W}$ and $2.5 \mathrm{~W}$. The data in Fig. 4.9 were taken in our first generation dual-beam trap with a probe beam (Fig. 3.11). The red
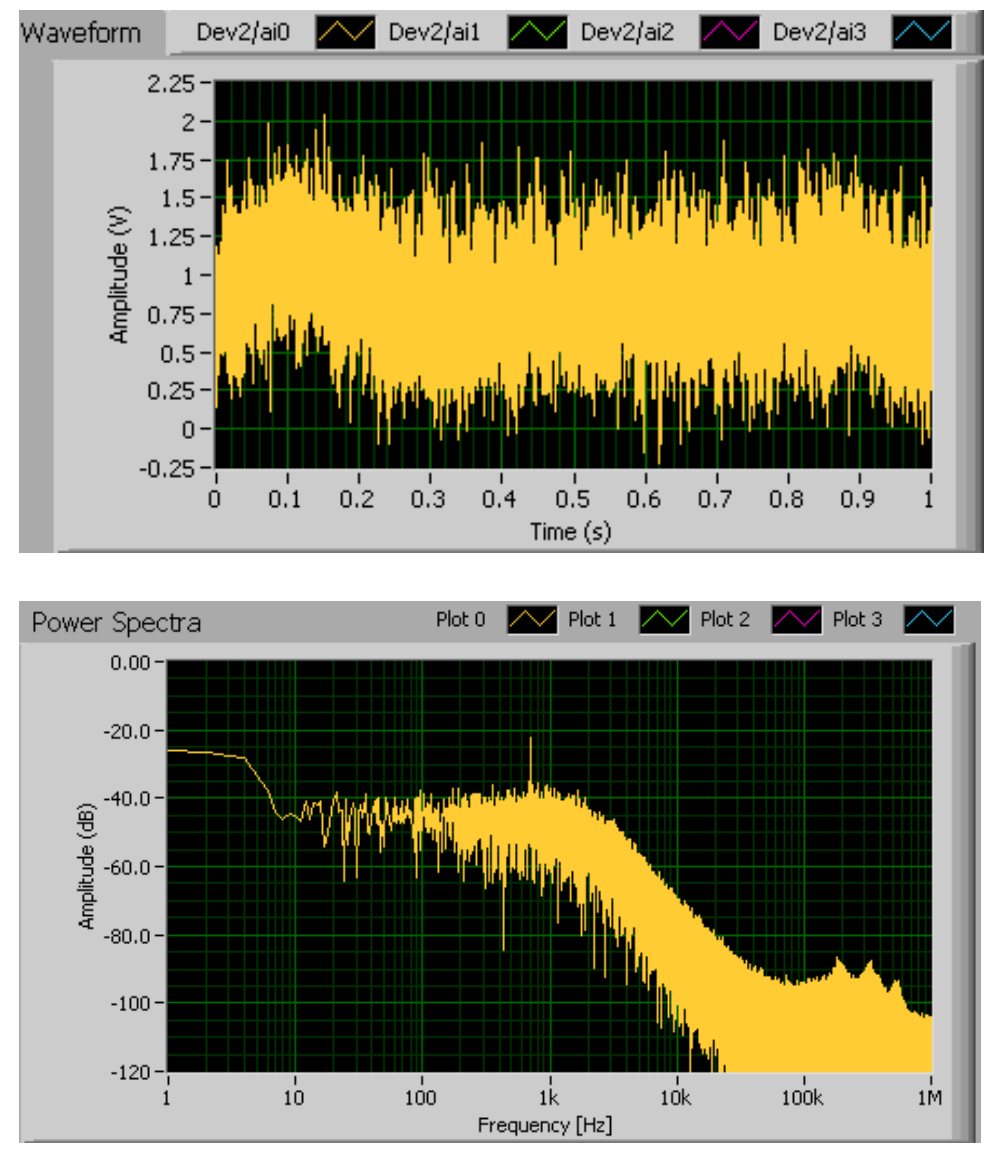

Figure 4.8: A waveform and spectrum of multiple (2 or maybe 3) 3.0- $\mu$ m beads trapped at 752 torr. The powers of the two trapping beams are $119 \mathrm{~mW}$ and $100 \mathrm{~mW}$. 
curves are for the motion in the horizontal direction, and the blue curves are for the motion in the vertical direction. Under certain conditions, we can even observe such narrow peaks at $1 \mathrm{~atm}$. It seams that the scattering forces from the trapping laser pump energy to the motion and amplify the motion.
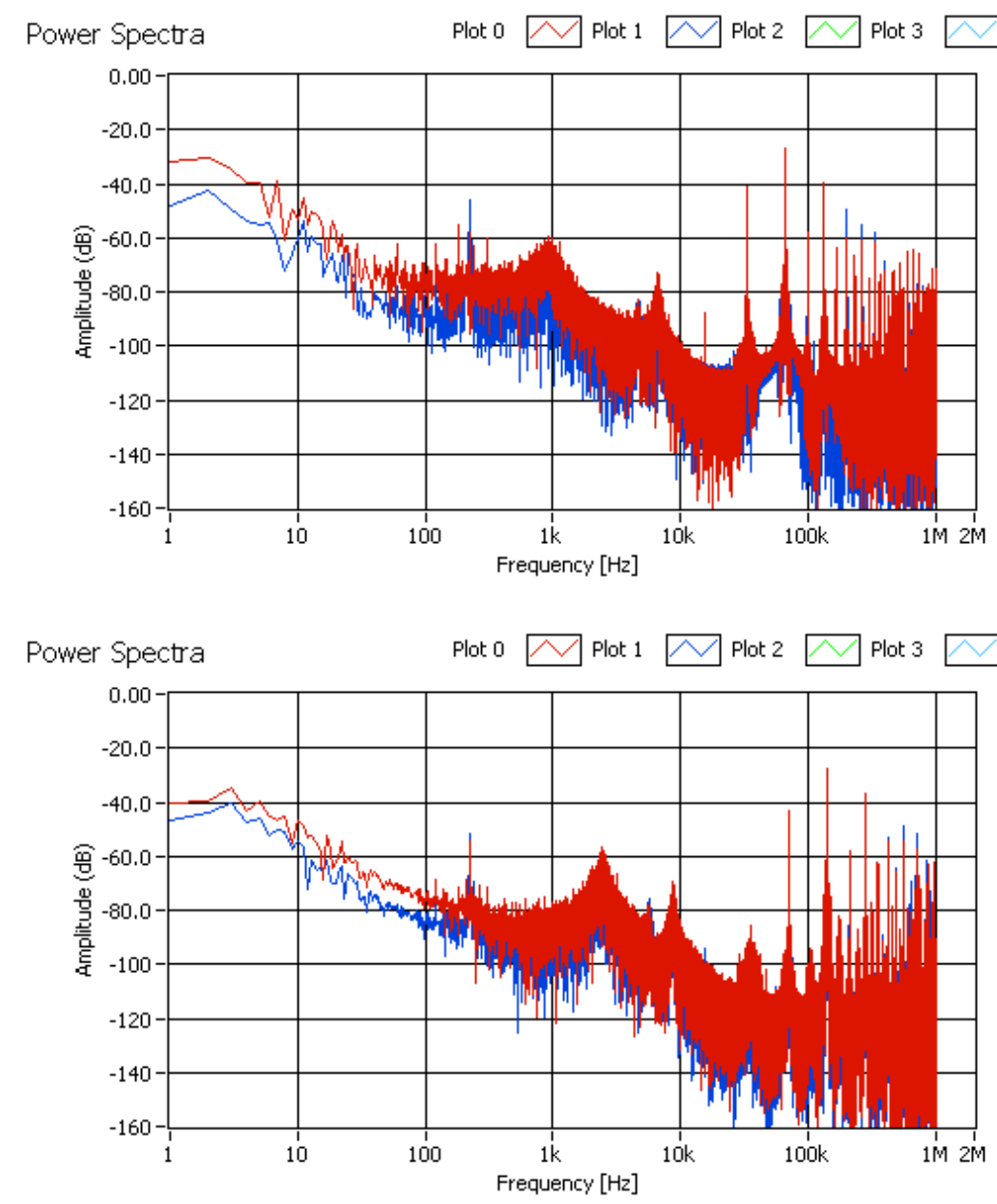

Figure 4.9: Spectra of two 4.7- $\mu \mathrm{m}$ beads trapped at 9.78 torr with different laser powers. The powers of the two trapping beams are $0.7 \mathrm{~W}$ and $1.0 \mathrm{~W}$ for the top figure, and $2.0 \mathrm{~W}$ and $2.5 \mathrm{~W}$ for the bottom figure. 


\subsection{Measurement of the instantaneous velocity of a Brow- nian particle in air}

A simplified scheme of our setup for measuring the instantaneous velocity of Brownian particle in air is shown in Fig. 4.4, and a more detailed optical circuit is shown in Fig. 4.5. The trap is formed inside a vacuum chamber by two counter-propagating laser beams focused to the same point by two identical aspheric lenses with focal length of $3.1 \mathrm{~mm}$ and numerical aperture of 0.68 . The two $1064 \mathrm{~nm}$ laser beams are orthogonally polarized, and their frequencies differ by $160 \mathrm{MHz}$ to avoid interference.

The two laser beams are aligned with the help of a pinhole aperture whose diameter is $1.0 \pm 0.5 \mu \mathrm{m}$. Because the pinhole has a finite thickness $(13 \mu \mathrm{m})$, it is difficult to align the foci of the two beams to the same point in the axial direction. We intentionally make the waist of one beam larger than the other to make this alignment less critical. The measured waists of the two beams are $2.2 \mu \mathrm{m}$ and $3.0 \mu \mathrm{m}$ in the horizontal direction. The real waists

should be smaller than these values due to the finite size of the pinhole. Also note that the waists of the two beams are measured at different axial positions separated by the thickness of the pinhole. Once a bead is trapped, we keep the power of one beam constant, and tune the power of the other beam to maximize the trapping frequency.

When the bead deviates from the center of the trap, it deflects both trapping beams. We monitor the position of the bead by measuring the deflection of one of the beams, which is split by a mirror with a sharp edge. 
The difference between the two halves is measured by a fast balanced detector. This simple, yet novel, detection scheme has a bandwidth of $75 \mathrm{MHz}$ and ultra-low noise $[28,78]$.

For the data included in this section, unless otherwise stated, the powers of the two laser beams are $10.7 \mathrm{~mW}$ and $14.1 \mathrm{~mW}$, the diameter of the bead is $3 \mu \mathrm{m}$, the temperature of the system is $297 \mathrm{~K}$, and the air pressure is $99.8 \mathrm{kPa}$ (749 torr) or $2.75 \mathrm{kPa}$ (20.6 torr). The trapping is very stable, and the heating due to laser absorption is negligible at these conditions. Examples of power spectra of a $3 \mu \mathrm{m}$ microsphere trapped at these conditions have been shown in Fig. 4.6 and Fig. 4.7.

The position signal of a trapped bead is recorded at a sampling rate of $2 \mathrm{MHz}$. Because of the limited spatial resolution, we are not able to obtain accurate instantaneous velocities of a bead at this rate. To reduce the noise, we average every 10 successive position measurements, and use these averages to calculate instantaneous velocities with time resolution of $5 \mu \mathrm{s}$. Although this method reduces the temporal resolution by a factor of 10 , it greatly increases the signal-to-noise ratio if both the trapping period $\left(2 \pi / \omega_{0}\right)$ and momentum relaxation time are much larger than $5 \mu \mathrm{s}$. These conditions are satisfied here since the trapping period is about $320 \mu \mathrm{s}, \tau_{p}=48 \mu \mathrm{s}$ at $99.8 \mathrm{kPa}$, and $\tau_{p}=147 \mu \mathrm{s}$ at $2.75 \mathrm{kPa}$.

Figure 4.10 shows typical samples of position and velocity traces of a trapped bead. The position traces of the bead at these two pressures appear very similar. On the other hand, the velocity traces are clearly different. The 
instantaneous velocity of the bead at $99.8 \mathrm{kPa}$ changes more frequently than that at $2.75 \mathrm{kPa}$, because the momentum relaxation time is shorter at higher pressure.

Figure 4.11 shows the mean square displacements of a $3 \mu \mathrm{m}$ silica bead as a function of time. The measured MSD's fit excellently with Eq. 4.17 over three decades of time for both pressures. The calibration factor $\alpha=$ position/voltage of the detection system is the only fitting parameter
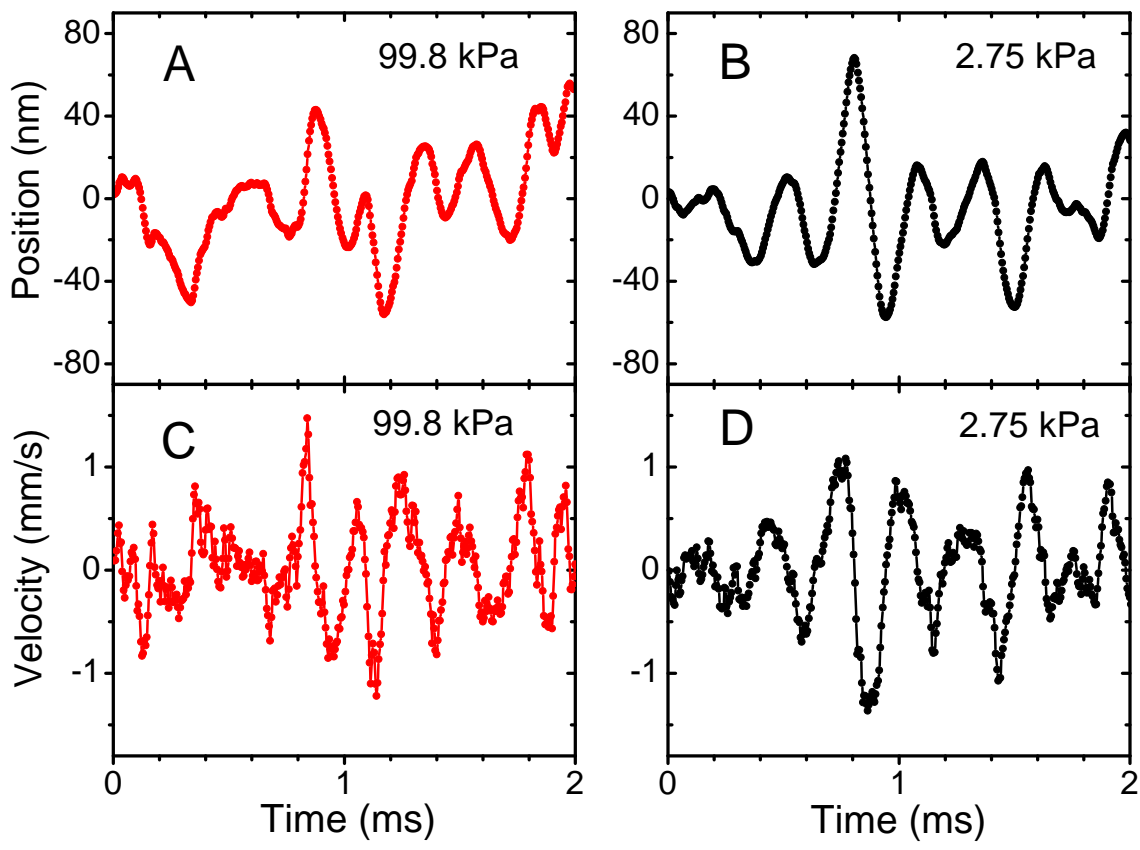

Figure 4.10: One-dimensional trajectories of a $3 \mu \mathrm{m}$ diameter silica bead trapped in air at $99.8 \mathrm{kPa}(\mathbf{A})$ and at $2.75 \mathrm{kPa}(\mathbf{B})$. The instantaneous velocities of the bead corresponding to these trajectories are shown in $(\mathbf{C})$ and (D). 

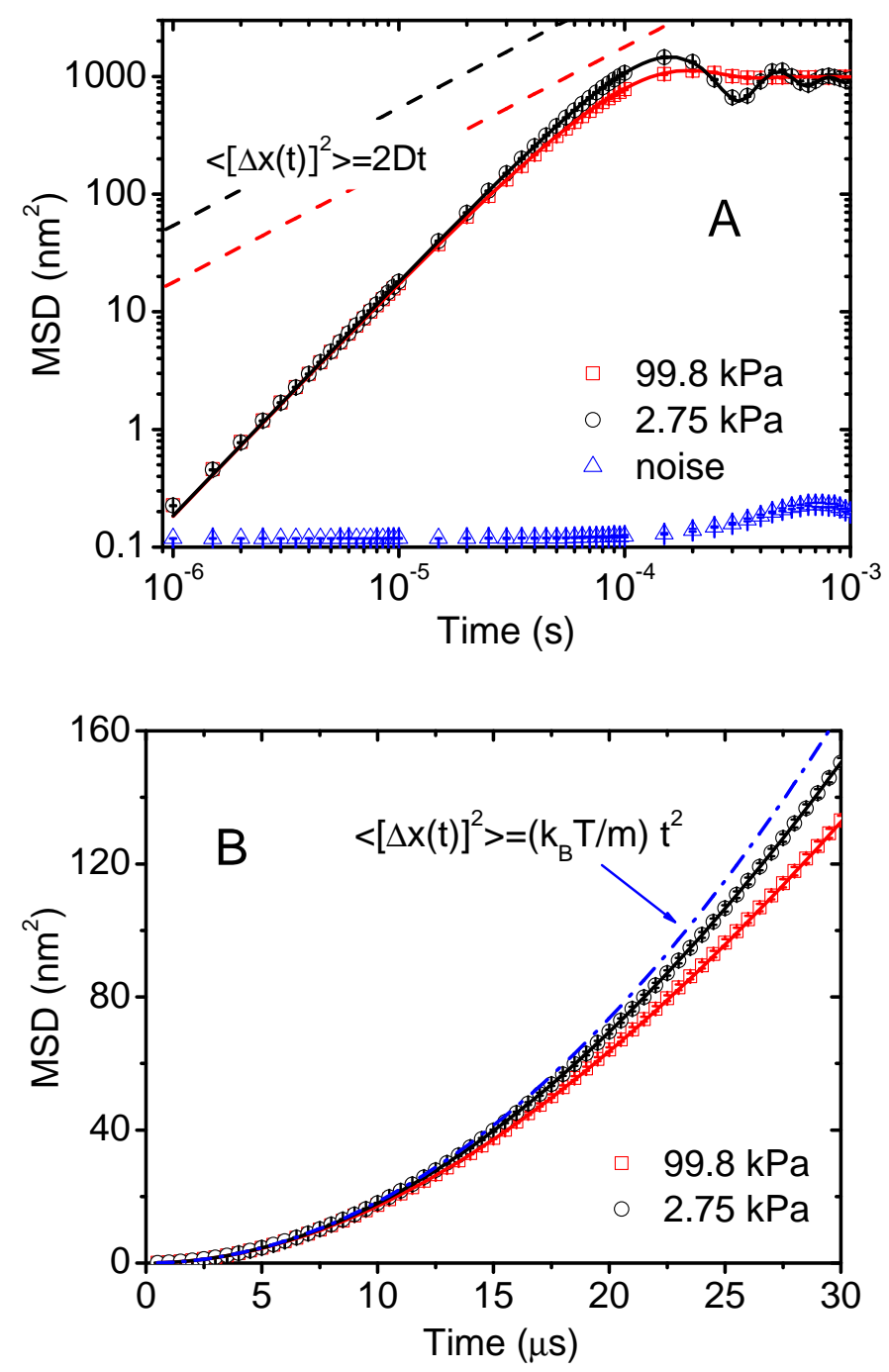

Figure 4.11: (A) The mean square displacements of a $3 \mu \mathrm{m}$ silica bead trapped in air at $99.8 \mathrm{kPa}$ (red square) and $2.75 \mathrm{kPa}$ (black circle). They are calculated from 40 million position measurements for each pressure. The "noise" signal (blue triangle) is recorded when there is no particle in the optical trap. The solid lines are the theoretical predictions of Eq. 4.17. They fit with the measurements excellently. The prediction of Einstein's theory of free Brownian motion in the diffusive regime is shown in dashed lines for comparison. (B) MSD's at short time scales are shown in detail. The dash-dot line indicates ballistic Brownian motion of a free particle. 
of Eq. 4.17 for each pressure. $M$ is calculated from the size and density of the microsphere. $\tau_{p}$ and $\Omega$ are obtained from the measured normalized velocity autocorrelation function (VACF). The two $\alpha$ 's obtained for these two pressures differ by $10.8 \%$. This is because the vacuum chamber is distorted slightly when the pressure is decreased from $99.8 \mathrm{kPa}$ to $2.75 \mathrm{kPa}$. One may avoid this problem in future by coupling the trapping laser into the vacuum chamber with an optical fiber and a teflon feedthrough [79]. The measured MSD's are completely different from those predicted by Einstein's theory of Brownian motion in a diffusive regime. The slopes of measured MSD curves at short time scales are double of those of the MSD curves of diffusive Brownian motion in the log-log plot (Fig. 4.11A). This is because the MSD is proportional to $t^{2}$ for ballistic Brownian motion, and it is proportional to $t$ for diffusive Brownian motion. Another important feature is that the MSD curves are independent of air pressure at short time scales, as is predicted by $\left\langle[\Delta x(t)]^{2}\right\rangle=\left(k_{B} T / M\right) t^{2}$ for ballistic Brownian motion, whereas the MSD in the diffusive regime does depend on the air pressure. At long time scales, the MSD saturates at a constant value because of the optical trap. Fig. 4.11B displays more detail of the Brownian motion at short time scales. It clearly demonstrates that we have observed ballistic Brownian motion.

The distributions of the measured instantaneous velocities are displayed in Fig. 4.12. They agree very well with the Maxwell-Boltzmann distribution. The measured rms velocities are $v_{r m s}=0.422 \mathrm{~mm} / \mathrm{s}$ at $99.8 \mathrm{kPa}$ and $v_{r m s}=$ $0.425 \mathrm{~mm} / \mathrm{s}$ at $2.75 \mathrm{kPa}$. These are very close to the prediction of the energy 
equipartition theorem, $v_{r m s}=\sqrt{k_{B} T / M}$, which is $0.429 \mathrm{~mm} / \mathrm{s}$. As expected, the velocity distribution is independent of pressure. The rms value of the noise signal is $0.021 \mathrm{~mm} / \mathrm{s}$, which means we have $1.0 \AA$ spatial resolution in $5 \mu \mathrm{s}$. This measurement noise is about $4.8 \%$ of the rms velocity. Fig. 4.12 represents direct verification of the Maxwell-Boltzmann distribution of velocities and the equipartition theorem of energy for Brownian motion. For a Brownian particle

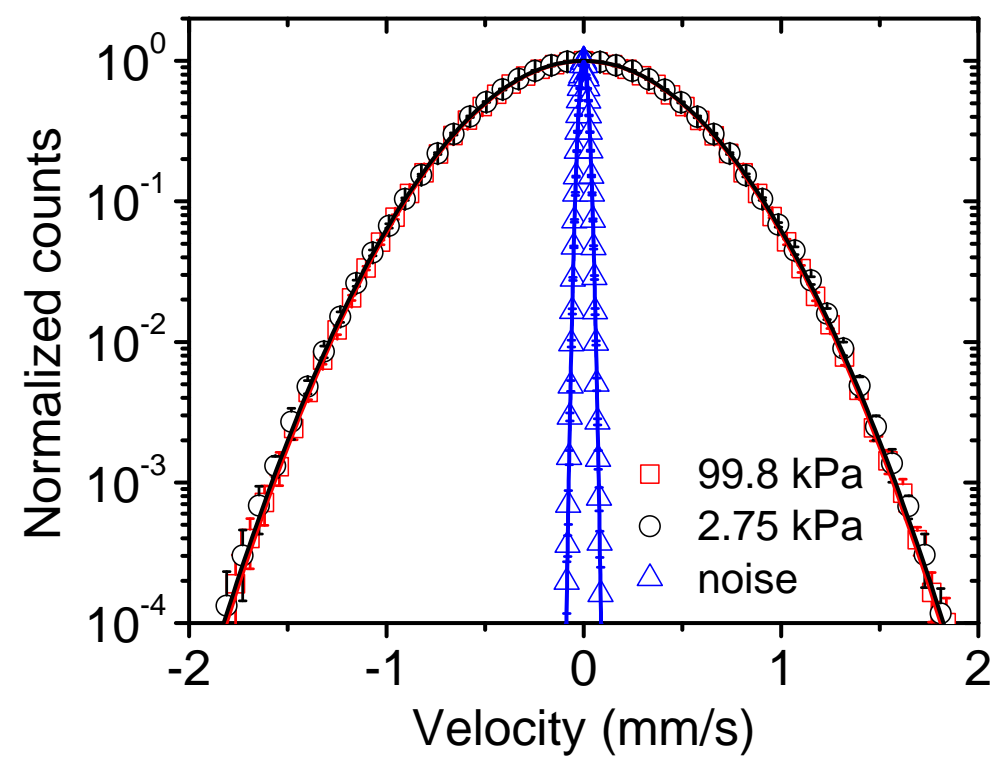

Figure 4.12: The distribution of the measured instantaneous velocities of a 3 $\mu \mathrm{m}$ silica bead. The statistics at each pressure are calculated from 4 million instantaneous velocities. The solid lines are Maxwell-Boltzmann distributions. We obtained $v_{r m s}=0.422 \mathrm{~mm} / \mathrm{s}$ at $99.8 \mathrm{kPa}$ (red square) and $v_{r m s}=0.425$ $\mathrm{mm} / \mathrm{s}$ at $2.75 \mathrm{kPa}$ (black circle) from the measurements. The rms value of the noise (blue triangle) is $0.021 \mathrm{~mm} / \mathrm{s}$, which means we have $1.0 \AA$ spatial resolution in $5 \mu \mathrm{s}$. 
in liquid, the inertial effects of the liquid become important. The measured rms velocity of the particle will be $v_{r m s}=\sqrt{k_{B} T / M^{*}}$ in the ballistic regime, where the effective mass $M^{*}$ is the sum of the mass of the particle and half the mass of the displaced fluid [80]. In order to measure the true instantaneous velocity in liquid as predicted by the equipartition theorem, the temporal resolution must be much shorter than the time scale of acoustic damping, which is less than $1 \mathrm{~ns}$ for a $1 \mu \mathrm{m}$ particle in liquid [80].

Figure 4.13 shows the normalized VACF of the bead at two different pressures. They fit with Eq. 4.20 nicely. At $2.75 \mathrm{kPa}$, one can clearly see

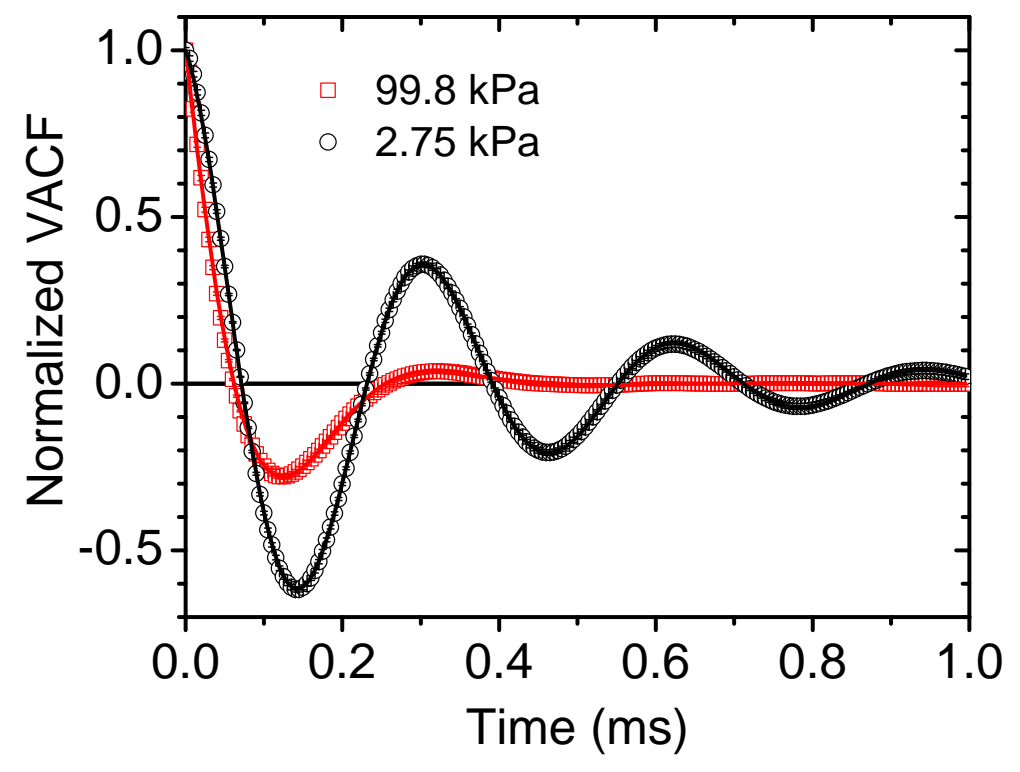

Figure 4.13: The normalized velocity autocorrelation functions of the $3 \mu \mathrm{m}$ bead at $99.8 \mathrm{kPa}$ (red square) and at $2.75 \mathrm{kPa}$ (black circle) from the measurements. The solid lines are fittings with Eq. 4.20. 
the oscillations due to the optical trap. Eq. 4.20 is independent of the calibration factor $\alpha$ of the detection system. The only independent variable is time $t$, which we can measure with high precision. Thus the normalized VACF provides an accurate method to measure $\tau_{p}$ and $\omega_{0}$. Fitting the normalized VACF with Eq. 4.20, we obtained $\tau_{p}=48.5 \pm 0.1 \mu \mathrm{s}, \omega_{0}=2 \pi \cdot(3064 \pm 4) \mathrm{Hz}$ at $99.8 \mathrm{kPa}$, and $\tau_{p}=147.3 \pm 0.1 \mu \mathrm{s}, \omega_{0}=2 \pi \cdot(3168 \pm 0.5) \mathrm{Hz}$ at $2.75 \mathrm{kPa}$. The trapping frequency changed by $3 \%$ due to the distortion of the vacuum chamber at different pressures. We can also calculate the diameter of the silica bead from the $\tau_{p}$ value at $99.8 \mathrm{kPa}$ [81]. The obtained diameter for this microsphere is $2.79 \mu \mathrm{m}$. This is within the uncertainty range given by the supplier of $3.0 \mu \mathrm{m}$ silica beads. We use this value in the calculation of MSD and normalized VACF.

For a particle at a certain pressure and temperature, $\tau_{p}$ should be independent of the trapping frequency. We verified this by changing the total power of the two laser beams from $25 \mathrm{~mW}$ to $220 \mathrm{~mW}$. The measured $\tau_{p}$ of a microsphere trapped at 19.6 torr and 749 torr as a function of the total laser power is shown in Fig. 4.14. Each data point has a few percent of uncertainty because we use a smaller data sets to calculate the $\tau_{p}$ than those used in the previous figures. Although the data points for each pressure are not perfectly on a line, it is clear that the $\tau_{p}$ 's are independent of the laser power within the experimental uncertainty. Fitting the data for each pressure with a straight line, we obtain $\tau_{p}=[151.3+0.00168 P /(1 \mathrm{~mW})] \mu$ s at 19.6 torr, and $\tau_{p}=[53.74+0.00275 P /(1 \mathrm{~mW})] \mu$ s at 749 torr for this microsphere, where $P$ 
is the total power of the two trapping beams. Thus $\tau_{p}$ changed less than $1.3 \%$ for both pressures when the total laser power is changed from 0 to $200 \mathrm{~mW}$. This proves that the fitting method is very accurate, and the heating due to the laser beams (which would change the viscosity and affect $\tau_{p}$ ) is negligible at these pressures.

In conclusion, we have observed the Brownian motion of a single particle in the ballistic regime, and measured its instantaneous velocity successfully for the first time. The ability to measure the instantaneous velocity of a Brownian particle will be invaluable in studying nonequilibrium statistical mechanics

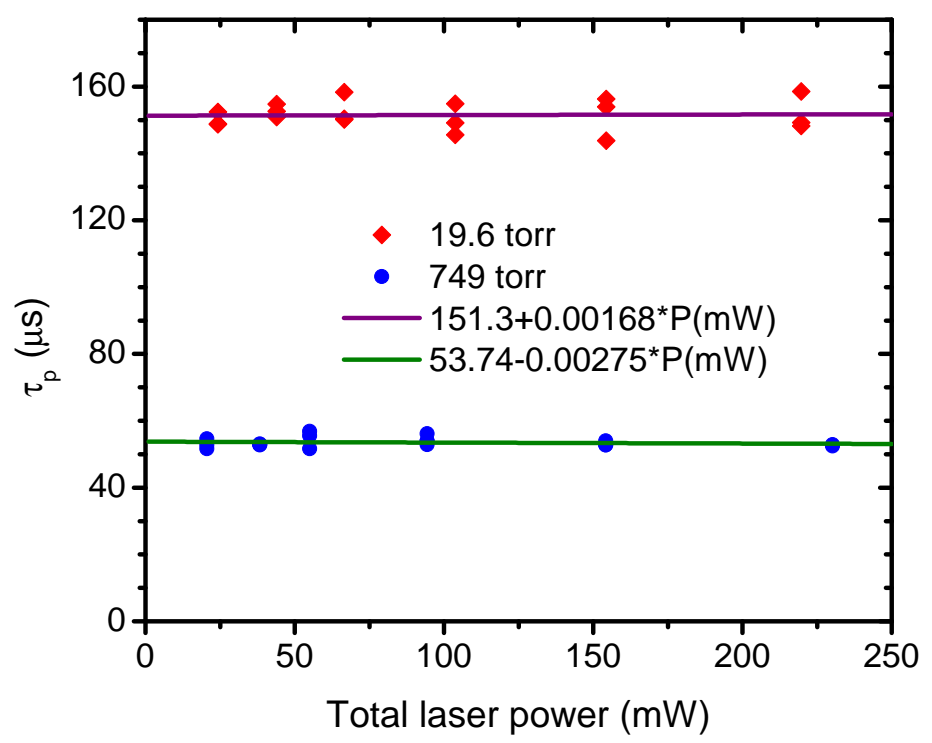

Figure 4.14: Measured momentum relaxation times $\left(\tau_{p}\right)$ of a microsphere trapped at 19.6 torr and 749 torr as a function of the total power of the two trapping beams. 
$[34,35]$ and can be used to cool Brownian motion by applying a feedback force with a direction opposite to the velocity $[82,83]$.

In vacuum, our optically trapped particle promises to be an ideal system for investigating quantum effects in a mechanical system $[45,78]$, due to its near-perfect isolation from the thermal environment. Combining feedback cooling and cavity cooling, we expect to cool the Brownian motion of a bead starting from room temperature to the quantum regime, as predicted by recent theoretical calculations $[17,18]$. We have directly verified the energy equipartition theorem of Brownian motion. However, we also expect to observe deviation from this theorem when the bead is cooled to the quantum regime. The kinetic energy of the bead will not approach zero even at $0 \mathrm{~K}$ because of its zero-point energy. The rotational energy of the bead should also become quantized.

An optically trapped microsphere can also be used to probe the properties of superfluid helium. Liquid helium has many special properties. Its density is only $0.129 \mathrm{~g} / \mathrm{cm}^{3}$, and its refractive index is only 1.024 , which is close to that of air (Table A.1). Thus optical trapping of microspheres in liquid helium is similar to optical trapping of microspheres in air. A microsphere in superfluid helium will do Brownian motion due to collisions between the microsphere and quasiparticles, such as phonons and rotons [84-86]. Thus we can probe the properties of superfluid helium by studying the Brownian motion of an optically trapped microsphere in it. 


\section{Chapter 5}

\section{Towards measurement of the instantaneous velocity of a Brownian particle in water}

\subsection{Motivation}

In the previous chapter, we presented our measurement of the instantaneous velocity of a Brownian particle in air. A more interesting experiment would be to measure the instantaneous velocity of a Brownian particle in water (or other liquid). Brownian motion was first discovered in water, and it affects many aspects of the life of cells in water. More importantly, Brownian motion in water may reveal new physics that has not been explored before.

It is clear that the velocity distribution of a Brownian particle in air satisfies the Maxwell-Boltzmann distribution. Since the Maxwell-Boltzmann distribution is derived for an ideal gas (or a weakly interacting system), it is not clear whether it holds equally well in water. In water, a moving microsphere drags water along with it as it moves. If the moving microsphere is suddenly stopped, the water flow caused by the previous motion of the microsphere will drag the microsphere to keep it moving. Thus the presence of water adds a memory effect to the motion of the bead. The water will also add an effective mass to the microsphere, since accelerating the microsphere requires a force 
both on the microsphere and the water which it displaces as it moves.

\subsection{Hydrodynamic theories of Brownian motion}

\subsubsection{A free particle in water}

The effective mass of the microsphere in water is the sum of the mass of the microsphere and half of the mass of the displaced water [80,87]:

$$
M^{*}=M_{p}+\frac{1}{2} M_{f}
$$

where $M_{p}=(4 / 3) \pi R^{3} \rho_{p}$ is the mass of the microsphere, $M_{f}=(4 / 3) \pi R^{3} \rho_{f}$ is the mass of displaced water, $\rho_{p}$ is the density of the microsphere, and $\rho_{f}$ is the density of water. The energy equipartition theorem needs to be modified to:

$$
\frac{1}{2} M^{*}\left\langle v^{2}\right\rangle=\frac{1}{2} k_{B} T
$$

where $v$ is the velocity of the microsphere in one dimension. Thus the rms ve-

locity is $v_{r m s}=\sqrt{k_{B} T / M^{*}}$. Because of the memory effect of water, the velocity autocorrelation function (VACF) of a free particle will not be $\langle v(t) v(0)\rangle=$ $\frac{k_{B} T}{M} e^{-t / \tau_{p}}$ as in air, but $[88-91]$

$$
\frac{\langle v(t) v(0)\rangle}{k_{B} T / M^{*}}=\frac{\alpha_{+} e^{\alpha_{+}^{2} t} \operatorname{erfc}\left(\alpha_{+} \sqrt{t}\right)-\alpha_{-} e^{\alpha_{-}^{2} t} \operatorname{erfc}\left(\alpha_{-} \sqrt{t}\right)}{\alpha_{+}-\alpha_{-}},
$$

where

$$
\alpha_{ \pm}=\frac{3}{2} \cdot \frac{3 \pm\left(5-36 \tau_{p} / \tau_{f}\right)^{1 / 2}}{\tau_{f}^{1 / 2}\left(1+9 \tau_{p} / \tau_{f}\right)} .
$$

$\tau_{p}=M_{p} /(6 \pi \eta R)=\frac{2}{9} R^{2} \rho_{p} / \eta$ is the momentum relaxation time of the particle due to its own inertia, $\tau_{f}=R^{2} \rho_{f} / \eta$ characterizes the effect of water. Here $\eta$ is the viscosity of water and $R$ is the radius of the microsphere. 
At long time scales, Eq. 5.3 approaches

$$
\frac{\langle v(t) v(0)\rangle}{k_{B} T / M^{*}} \propto \frac{1}{t^{3 / 2}} \text { for } t \rightarrow \infty \text {. }
$$

At short time scales, Eq. 5.3 approaches

$$
\frac{\langle v(t) v(0)\rangle}{k_{B} T / M^{*}}=\exp \left(-b \sqrt{t / \tau_{f}}\right) \text { for } t \rightarrow 0,
$$

where

$$
b=\frac{18}{\sqrt{\pi}\left(1+2 \rho_{p} / \rho_{f}\right)} .
$$

For a silica microsphere in water, $b=2.03$. The normalized VACF approaches 1 at short time scales as $\sqrt{t}$, rather than $t$. This is very different from the case in air.

The mean square displacement of a free microsphere in water is [26, 92]:

$$
\left\langle[\Delta x(t)]^{2}\right\rangle_{\text {free }}=2 D t\left[1-2 \sqrt{\frac{1}{\pi} \frac{\tau_{f}}{t}}+\frac{8}{9} \frac{\tau_{f}}{t}-\frac{\tau_{p}}{t}+\Xi\left(\frac{\tau_{p}}{\tau_{f}}, \frac{t}{\tau_{f}}\right)\right],
$$

where $D=k_{B} T /(6 \pi \eta R)$ is the diffusion coefficient. $\Xi\left(\frac{\tau_{p}}{\tau_{f}}, \frac{t}{\tau_{f}}\right)$ is a correction term:

$$
\Xi\left(\frac{\tau_{p}}{\tau_{f}}, \frac{t}{\tau_{f}}\right)=\frac{3}{t\left(5 \tau_{f}-36 \tau_{p}\right)^{1 / 2}}\left(\frac{1}{\alpha_{+}^{3}} e^{\alpha_{+}^{2} t} \operatorname{erfc}\left(\alpha_{+} \sqrt{t}\right)-\frac{1}{\alpha_{-}^{3}} e^{\alpha_{-}^{2} t} \operatorname{erfc}\left(\alpha_{-} \sqrt{t}\right)\right) .
$$

The behavior of the MSD at the long time limit is:

$$
\left\langle[\Delta x(t)]^{2}\right\rangle=2 D t \quad \text { for } \quad t \gg \tau_{p}
$$

Recently, the velocity autocorrelation function of a Brownian particle in water was measured successfully for $\frac{\langle v(t) v(0)\rangle}{\left\langle v^{2}\right\rangle}<0.35[29,91]$. However, the 
instantaneous velocity of a Brownian particle in water will be much more difficult to measure than the velocity autocorrelation function, and has not been measured to date. This is because the velocity autocorrelation function is a statistical average, which is insensitive to the high-frequency noise in the measurement. In our experiment, we hope that we can measure the instantaneous velocity of a Brownian particle in water for the first time, with particular interest to test the modified Maxwell-Boltzmann velocity distribution:

$$
f_{v}\left(v_{i}\right)=\sqrt{\frac{M^{*}}{2 \pi k_{B} T}} \exp \left(-\frac{M^{*} v_{i}^{2}}{2 k_{B} T}\right) .
$$

\subsubsection{An optically trapped microsphere in water}

The optical trap provides a harmonic force $F_{\text {trap }}=-k x$ on the micro-

sphere when the displacement of the microsphere is small. $k=M_{p} \Omega^{2}$ where $\Omega$ is the natural angular frequency of the trap. Clercx and Schram [90] gave analytical solutions for the MSD and VACF of a trapped Brownian particle in a liquid, and Berg-Sørensen and Flyvbjerg [76] gave a solution for the power spectrum density (PSD) of a trapped Brownian particle in a liquid. This section introduces their analytical solutions and provides some numerical results to visualize those solutions. These numerical results will serve as a guide for our experiment.

Because the velocity of the Brownian motion of a microsphere in liquid is much smaller than the speed of sound in the liquid, we can describe the fluid motion by the linearized incompressible time-dependent Navier-Stokes equation. The Langevin equation of the motion of a trapped microsphere in a 
liquid is [90]:

$$
\begin{aligned}
M^{*} \ddot{x}(t)= & -k x(t)-6 \pi \eta R \dot{x}(t) \\
& -6 R^{2} \sqrt{\pi \rho_{f} \eta} \int_{-\infty}^{t}\left(t-t^{\prime}\right)^{-1 / 2} \ddot{x}\left(t^{\prime}\right) d t^{\prime}+F_{\text {therm }}(t) .
\end{aligned}
$$

The first term after the equal sign of Eq. 5.11 is the harmonic force, the second term is the ordinary Stokes's friction, the third term is a memory term associated with the hydrodynamic retardation effects of the liquid, and the last term is the Brownian stochastic force.

The mean-square displacement of a trapped microsphere in a liquid is $[90,92]$

$$
\begin{aligned}
\left\langle[\Delta x(t)]^{2}\right\rangle_{\text {trap }}= & \frac{2 k_{B} T}{k}+\frac{2 k_{B} T}{M^{*}}\left[\frac{e^{z_{1}^{2} t} \operatorname{erfc}\left(z_{1} \sqrt{t}\right)}{z_{1}\left(z_{1}-z_{2}\right)\left(z_{1}-z_{3}\right)\left(z_{1}-z_{4}\right)}\right. \\
& +\frac{e^{z_{2}^{2} t} \operatorname{erfc}\left(z_{2} \sqrt{t}\right)}{z_{2}\left(z_{2}-z_{1}\right)\left(z_{2}-z_{3}\right)\left(z_{2}-z_{4}\right)} \\
& +\frac{e^{z_{3}^{2} t} \operatorname{erfc}\left(z_{3} \sqrt{t}\right)}{z_{3}\left(z_{3}-z_{1}\right)\left(z_{3}-z_{2}\right)\left(z_{3}-z_{4}\right)} \\
& \left.+\frac{e^{z_{4}^{2} t} \operatorname{erfc}\left(z_{4} \sqrt{t}\right)}{z_{4}\left(z_{4}-z_{1}\right)\left(z_{4}-z_{2}\right)\left(z_{4}-z_{3}\right)}\right]
\end{aligned}
$$

The coefficients $z_{1}, z_{2}, z_{3}$, and $z_{4}$ are the four roots of the equation [92]

$$
\left(\tau_{p}+\frac{1}{9} \tau_{f}\right) z^{4}-\sqrt{\tau_{f}} z^{3}+z^{2}+\frac{1}{\tau_{k}}=0,
$$

where $\tau_{k}=6 \pi \eta R / k$. For $t \rightarrow \infty$, Eq. 5.12 approaches

$$
\left\langle[\Delta x(\infty)]^{2}\right\rangle_{\text {trap }}=\frac{2 k_{B} T}{k} .
$$




\begin{tabular}{|c|c|c|c|c|c|}
\hline Diameter & $k$ & $\tau_{p}$ & $\tau_{f}$ & $\tau_{k}$ & $\tau_{c}$ \\
\hline & & $\frac{2}{9} R^{2} \rho_{p} / \eta$ & $R^{2} \rho_{f} / \eta$ & $6 \pi \eta R / k$ & $R / c$ \\
\hline$(\mu \mathrm{m})$ & $(\mu \mathrm{N} / \mathrm{m})$ & $(\mu \mathrm{s})$ & $(\mu \mathrm{s})$ & $(\mu \mathrm{s})$ & $(\mathrm{ns})$ \\
\hline \hline 1.0 & 100 & 0.11 & 0.25 & 94 & 0.34 \\
\hline 3.0 & 33.3 & 1.0 & 2.2 & 851 & 1.01 \\
\hline 4.7 & 21.3 & 2.45 & 5.51 & 2083 & 1.58 \\
\hline 10 & 10 & 11.1 & 25.0 & 9443 & 3.4 \\
\hline
\end{tabular}

Table 5.1: Characteristic time scales of an optically trapped silica microsphere in water at $20{ }^{\circ} \mathrm{C}$. The second row shows the definitions of $\tau_{p, f, k, c}$. The second column $(k)$ is the spring constant of the optical trap. It is assumed to be inversely proportional to the diameter of the microsphere when the laser power is constant.

The normalized VACF of a trapped microsphere in a liquid is [90, 92]

$$
\begin{aligned}
A(t)=\frac{\langle v(t) v(0)\rangle}{k_{B} T / M^{*}}= & \frac{z_{1}^{3} e^{z_{1}^{2} t} \operatorname{erfc}\left(z_{1} \sqrt{t}\right)}{\left(z_{1}-z_{2}\right)\left(z_{1}-z_{3}\right)\left(z_{1}-z_{4}\right)} \\
& +\frac{z_{2}^{3} e^{z_{2}^{2} t} \operatorname{erfc}\left(z_{2} \sqrt{t}\right)}{\left(z_{2}-z_{1}\right)\left(z_{2}-z_{3}\right)\left(z_{2}-z_{4}\right)} \\
& +\frac{z_{3}^{3} e^{z_{3}^{2} t} \operatorname{erfc}\left(z_{3} \sqrt{t}\right)}{\left(z_{3}-z_{1}\right)\left(z_{3}-z_{2}\right)\left(z_{3}-z_{4}\right)} \\
& +\frac{z_{4}^{3} e^{z_{4}^{2} t} \operatorname{erfc}\left(z_{4} \sqrt{t}\right)}{\left(z_{4}-z_{1}\right)\left(z_{4}-z_{2}\right)\left(z_{4}-z_{3}\right)} .
\end{aligned}
$$

The power spectral density is $[76,92]$ :

$$
S(f)=\frac{D}{2 \pi^{2} f^{2}} \frac{1+\sqrt{f / 2 \phi_{f}}}{\left(\phi_{k} / f-\sqrt{f / 2 \phi_{f}}-f / \phi_{p}-f / 9 \phi_{f}\right)^{2}+\left(1+\sqrt{f / 2 \phi_{f}}\right)^{2}},
$$

where $f$ is the observation frequency, $\phi_{k}=1 /\left(2 \pi \tau_{k}\right)$ is the corner frequency of the power spectrum due to the trap, and $\phi_{p, f}=1 /\left(2 \pi \tau_{p, f}\right)$. For $f \rightarrow 0$, Eq. 5.15 approaches

$$
S(0)=\frac{2 k_{B} T \gamma}{k^{2}}
$$


where $\gamma=6 \pi \eta R$.

At $t \rightarrow 0$, Eq. 5.14 predicts $\langle v(0) v(0)\rangle=k_{B} T / M^{*}$, which is different from the energy equipartition theorem $\langle v(0) v(0)\rangle=k_{B} T / M_{p}$. This conflict is caused by the assumption in Eq. 5.11 that the liquid is incompressible. For $t<t_{c}$, we need to consider the liquid to be compressible. Here $t_{c}=R / c$ is the time required for a sound wave to travel a sphere radius, where $c$ is the speed of sound in the liquid. The normalized velocity autocorrelation function

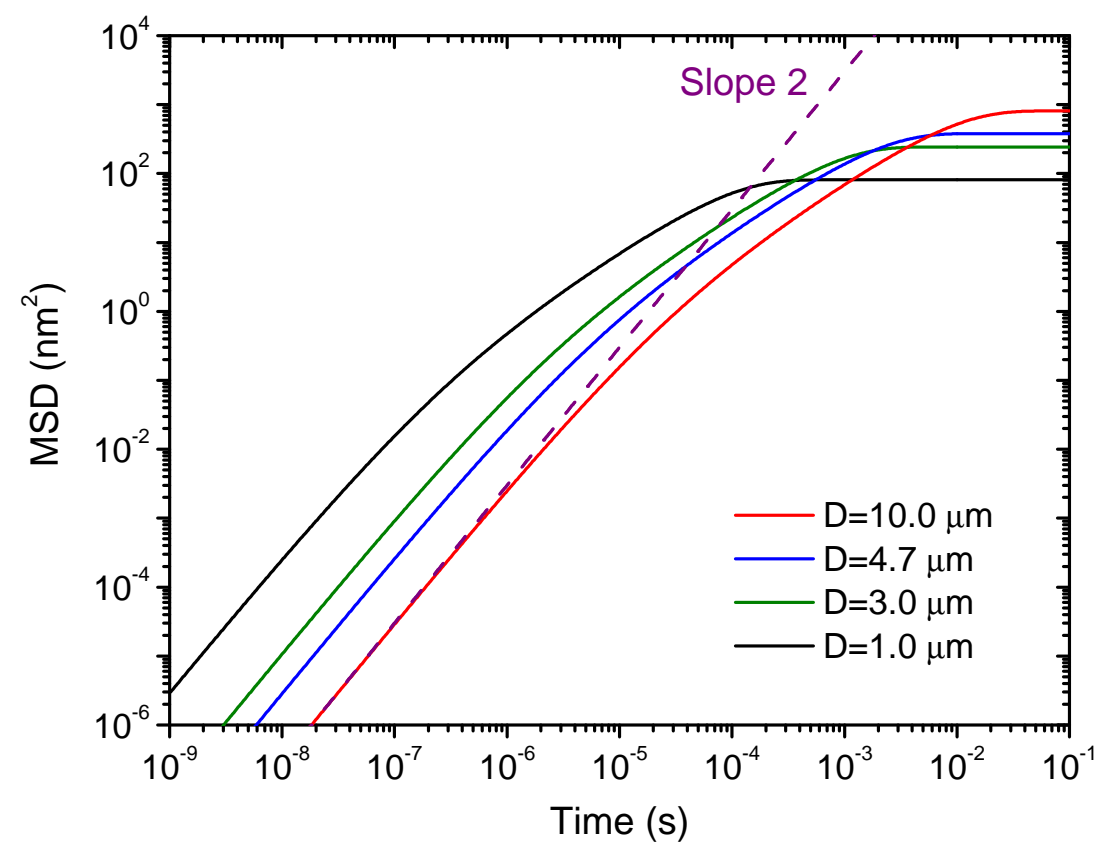

Figure 5.1: Mean square displacement of an optically trapped silica microsphere in water at $20^{\circ} \mathrm{C}$. Parameters are the same as those in Table 5.1. 
at $t \sim t_{c}$ is [80]:

$$
\begin{aligned}
A(t)=\frac{\langle v(t) v(0)\rangle}{k_{B} T / M^{*}}= & 1+\frac{M_{f}}{2 M_{p}}\left[\frac{1}{2}-\frac{i M^{*}}{\left(4 M_{p}^{2}-M_{f}^{2}\right)^{1 / 2}}\right] e^{-i x_{1} t / t_{c}} \\
& +\frac{M_{f}}{2 M_{p}}\left[\frac{1}{2}+\frac{i M^{*}}{\left(4 M_{p}^{2}-M_{f}^{2}\right)^{1 / 2}}\right] e^{-i x_{2} t / t_{c}},
\end{aligned}
$$

where

$$
\begin{aligned}
& x_{1}=-i \frac{M^{*}}{M_{p}}+\left[1-\frac{M_{f}^{2}}{4 M_{p}^{2}}\right]^{1 / 2}, \\
& x_{2}=-i \frac{M^{*}}{M_{p}}-\left[1-\frac{M_{f}^{2}}{4 M_{p}^{2}}\right]^{1 / 2} .
\end{aligned}
$$

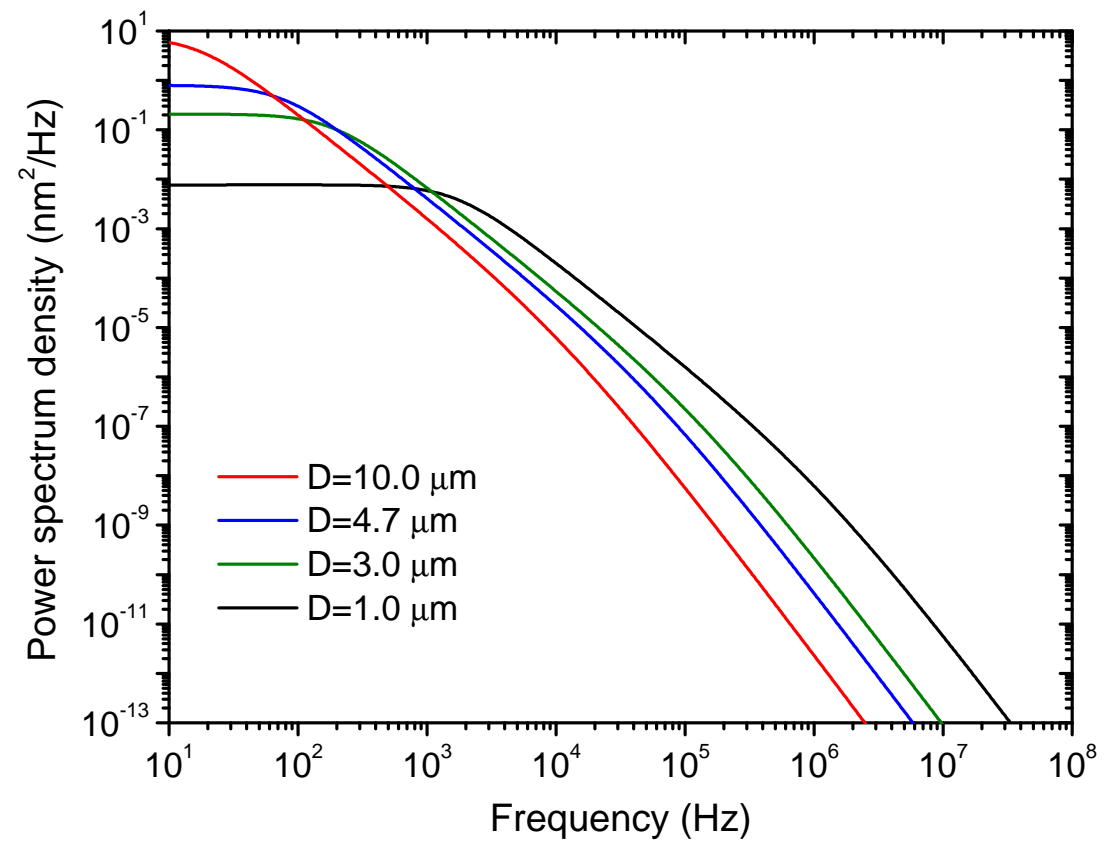

Figure 5.2: Power spectra of an optically trapped silica microsphere in water at $20^{\circ} \mathrm{C}$. Parameters are the same as those in Table 5.1. 
At very short time scales $t \ll t_{c}$, Eq. 5.16 approaches $A(0)=1+\frac{M_{f}}{2 M_{p}}$. The short time limit $A(0) \neq 1$ because the normalization factor is $k_{B} T / M^{*}$, rather than $k_{B} T / M_{p}$.

The MSD's of microspheres with different diameters in water are shown in Fig. 5.1, and the corresponding power spectra are shown in Fig. 5.2. Fig. 5.3 displays the normalized velocity autocorrelation function $(A(t))$ of an optically trapped silica microsphere in water at $20{ }^{\circ} \mathrm{C}$. Fig. $5.3(\mathrm{~A})$ displays the VACF's with a linear-log scale to cover a large range of time scales, and Fig. 5.3(B) displays the VACF's in a log-linear scale to show the details at short time scales. The thin solid lines at short time scales $\left(t<10^{-8} \mathrm{~s}\right)$ are calculated from Eq. 5.16, which includes the compressibility effects of water. The thick solid lines are calculated from Eq. 5.14, which treats the water as an incompressible fluid. The dashed lines are exponential decays with $\tau_{p}=1.0 \mu \mathrm{s}$, corresponding to a $3.0 \mu \mathrm{m}$ microsphere. As clearly shown in Fig. 5.3 , the VACF of a microsphere in water is very different from exponential decay because of the hydrodynamic memory effects of water.

The thin solid lines are expected to be correct for $t \sim t_{c}$, and the thick sold lines are expected to be correct for $t \gg t_{c}$. The intermediate regime $t_{c}<t<100 t_{c}$ is still poorly understood. It is suspicious that the thick solid curves approaches 1 so slowly at the short time limit. The normalized VACF a $4.7 \mu \mathrm{m}$ diameter silica microsphere in water at $20{ }^{\circ} \mathrm{C}$ and $60{ }^{\circ} \mathrm{C}$ is shown in linear-linear scales in Fig. 5.4. The VACF follows $\exp \left(-b \sqrt{t / \tau_{f}}\right)$ at the short time limit, rather than $\exp \left(-t / \tau_{p}\right)$. A recent experiment has measured the 

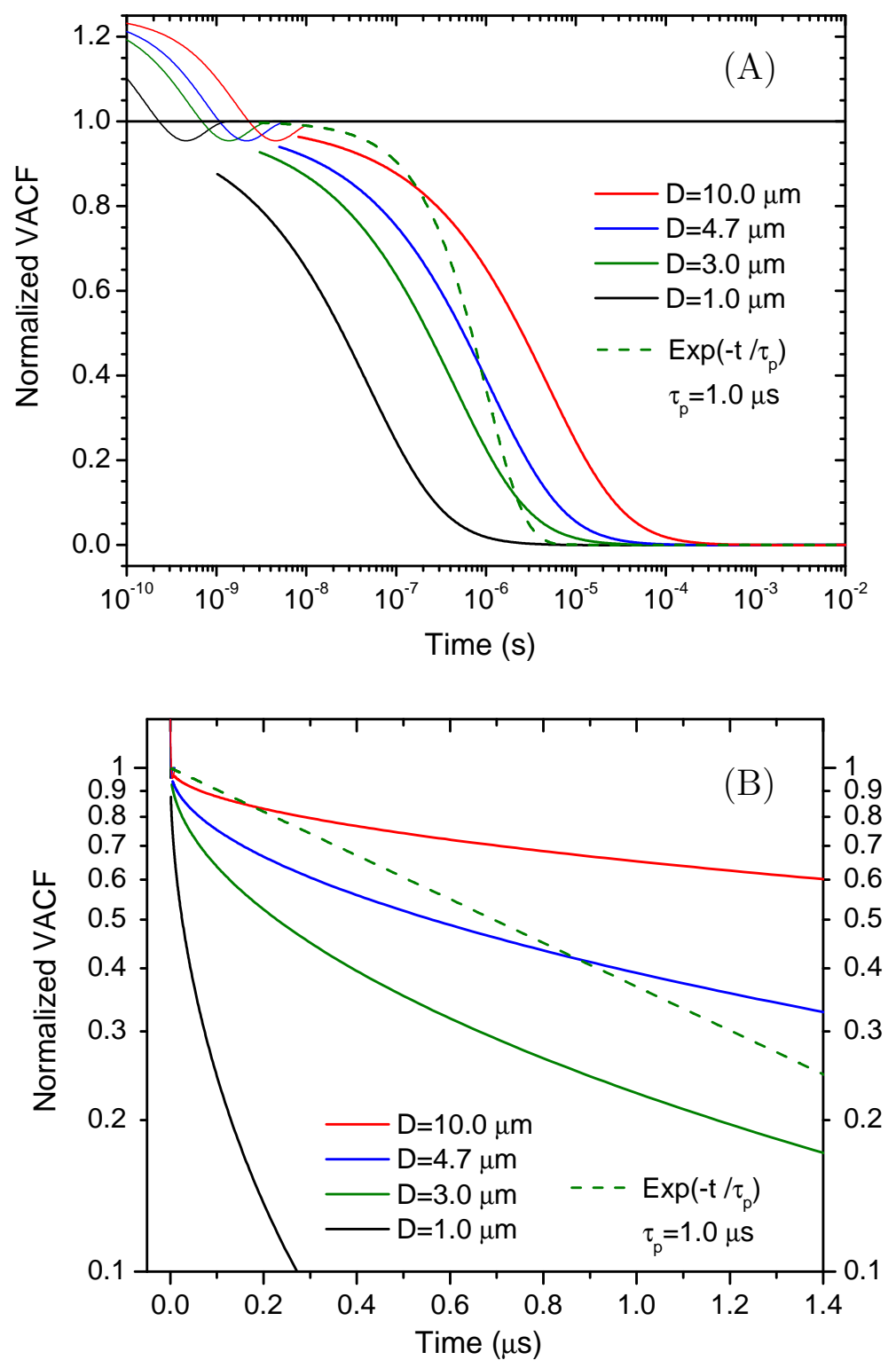

Figure 5.3: Normalized velocity autocorrelation function of an optically trapped silica microsphere in water at $20{ }^{\circ} \mathrm{C}$. Parameters are the same as those in Table 5.1. (A) covers large range of time scales, and (B) shows the details at short time scales. The thin solid lines $\left(t<10^{-8} \mathrm{~s}\right)$ are calculated from Eq. 5.16, and the thick solid lines are calculated from Eq. 5.14. The dashed lines are exponential decays with $\tau_{p}=1.0 \mu \mathrm{s}$, corresponding to a 3.0 $\mu \mathrm{m}$ microsphere. 
$\mathrm{VACF}$ of a Brownian particle in water at $\mathrm{VACF}<0.35$ [29]. A measurement of the VACF between 1 and 0.35 is required in order to better understand the hydrodynamic effects and compressibility effects of water on Brownian motion [93].

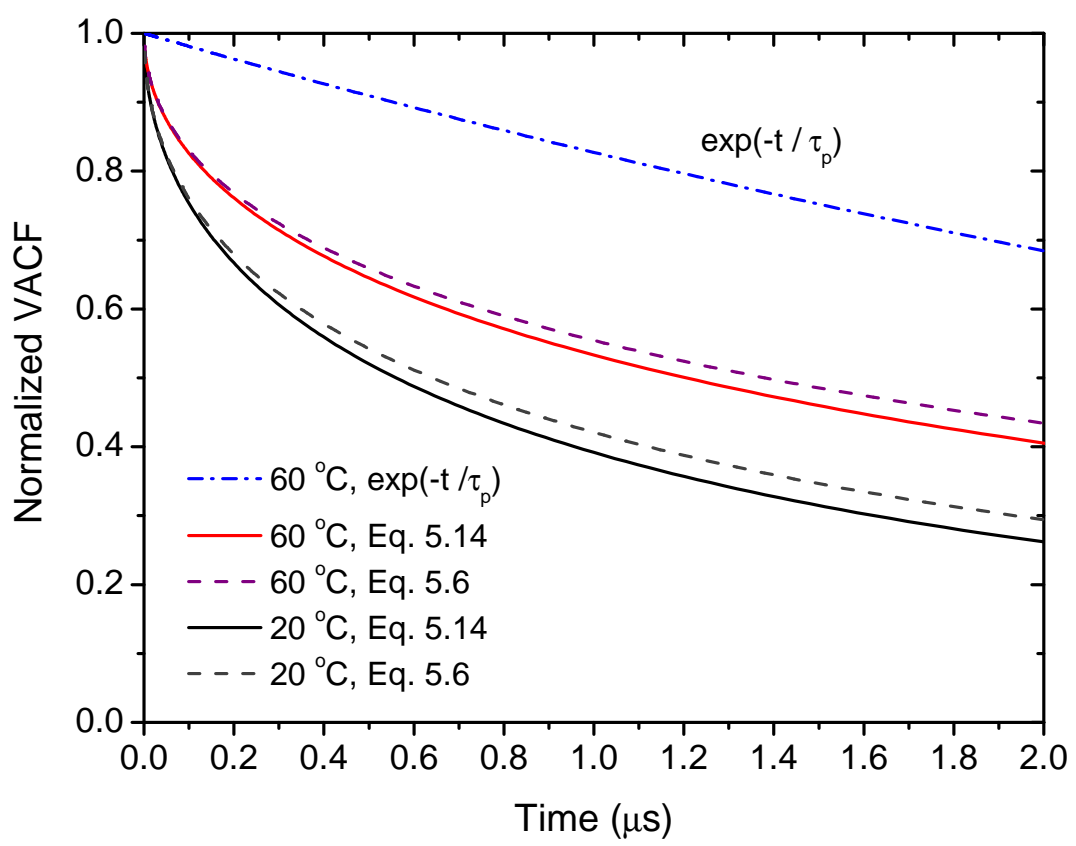

Figure 5.4: Normalized velocity autocorrelation function of a $4.7 \mu \mathrm{m}$ diameter silica microsphere in water at $20{ }^{\circ} \mathrm{C}$ and $60{ }^{\circ} \mathrm{C}$. The solid lines are exact results of the hydrodynamic theory of Brownian motion in a liquid (Eq. 5.14). The dashed lines are $\exp \left(-b \sqrt{t / \tau_{f}}\right)$, which is the short time limit of the hydrodynamic theory (Eq. 5.6). The dashed dot line is an exponential decay $\exp \left(-t / \tau_{p}\right)$. 


\subsection{Requirements for measuring the instantaneous ve- locity}

In order to measure the instantaneous velocity of a Brownian particle in water, the temporal resolution of the detection system must be much shorter than the momentum relaxation time of the particle, and the spatial resolution of the detection system must be much smaller than the displacement of the particle during the measurement time.

Because of the inertia of the particle and the water, the effective momentum relaxation time is

$$
\tau_{p}^{*}=\frac{M^{*}}{6 \pi \eta R}
$$

In order to resolve the instantaneous velocity with $10 \%$ uncertainty, we need the temporal resolution to be at least $\Delta t=\tau_{p}^{*} / 10$. The average displacement of the microsphere during $\Delta t$ is $\tau_{p}^{*} v_{r m s} / 10$. Thus the required spatial resolution is $\Delta x=\tau_{p}^{*} v_{r m s} / 100$. The sensitivity of a detector is usually characterized by its noise spectral density $s_{x}$. The spectral density of the detector should

satisfy $s_{x}<\Delta x \sqrt{\Delta t}$ at the required frequency $1 / \Delta t$ in order to measure the instantaneous velocity.

Table 5.2 shows the minimum required resolution for measuring the instantaneous velocity of a Brownian particle in water. To measure the instantaneous velocity of a $1 \mu \mathrm{m}$ diameter microsphere in water at $20^{\circ} \mathrm{C}$, the noise spectral density of the detection system should be smaller than $0.28 \mathrm{fm} / \mathrm{Hz}^{1 / 2}$ around $71 \mathrm{MHz}$. The requirement is relaxed to $0.94 \mathrm{fm} / \mathrm{Hz}^{1 / 2}$ around $34 \mathrm{MHz}$ if the temperature of the water is increased to $60{ }^{\circ} \mathrm{C}$. This is mainly because 


\begin{tabular}{|c|ccc|ccc|}
\hline & \multicolumn{3}{|c|}{$20^{\circ} \mathrm{C}$} & \multicolumn{3}{c|}{$60^{\circ} \mathrm{C}$} \\
\hline $\begin{array}{c}\text { Diameter } \\
(\mu \mathrm{m})\end{array}$ & $\begin{array}{c}\Delta t \\
(\mu \mathrm{s})\end{array}$ & $\begin{array}{c}\Delta x \\
(\mathrm{pm})\end{array}$ & $\begin{array}{c}s_{x} \\
\left(\mathrm{fm} / \mathrm{Hz}^{1 / 2}\right)\end{array}$ & $\begin{array}{c}\Delta t \\
(\mu \mathrm{s})\end{array}$ & $\begin{array}{c}\Delta x \\
(\mathrm{pm})\end{array}$ & $\begin{array}{c}s_{x} \\
\left(\mathrm{fm} / \mathrm{Hz}^{1 / 2}\right)\end{array}$ \\
\hline \hline 1.0 & 0.014 & 2.4 & 0.28 & 0.029 & 5.5 & 0.94 \\
1.9 & 0.049 & 3.3 & 0.74 & 0.10 & 7.6 & 2.5 \\
3.0 & 0.12 & 4.2 & 1.5 & 0.26 & 9.6 & 4.9 \\
4.7 & 0.30 & 5.2 & 2.9 & 0.65 & 12 & 9.6 \\
10 & 1.4 & 7.6 & 8.9 & 2.9 & 17 & 30 \\
\hline
\end{tabular}

Table 5.2: Minimum required detection resolution for measuring the instantaneous velocity of a Brownian particle in water at $20{ }^{\circ} \mathrm{C}$ and $60{ }^{\circ} \mathrm{C}$.

the viscosity of the water decreases when the temperature increases (Table A.1). Thus it is easier to measure the instantaneous velocity at $60{ }^{\circ} \mathrm{C}$ than at $20{ }^{\circ} \mathrm{C} . t_{p}^{*}$ is bigger for a larger particle, so the requirements for measuring the instantaneous velocity of a large microsphere is less demanding than that of a small microsphere. However, the sensitivity of the detection system varies for microspheres of different size. Thus there is an optimal size for measuring the instantaneous velocity.

The resolution of particle tracking with an optical tweezer is fundamentally limited by the shot noise of the laser. Because the size of the microsphere is on the same order as the wavelength of the laser, an accurate calculation of the shot noise limited sensitivity is very complex. Here we only discuss a simple estimation. The shot noise limited noise spectral density is estimated to be $[78]$

$$
s_{x} \approx 5 \times 10^{-16} G\left(\frac{R}{1 \mu \mathrm{m}}\right)\left(\frac{100 \mathrm{~mW}}{P}\right)^{1 / 2}\left(\frac{1 \mu \mathrm{m}}{\lambda}\right)^{1 / 2} \mathrm{~m} / \sqrt{\mathrm{Hz}}
$$

where $G$ is a geometrical factor that depends on $R / \lambda, R / w$, and the precise 
details of the Mie scattering by the sphere. $\lambda$ is the wavelength of the laser, $w$ is the waist of the laser beam, and $P$ is the power of the laser.

If the wavelength of the laser is $1 \mu \mathrm{m}$ and the power is $100 \mathrm{~mW}$, we need $G<1.1$ to measure the instantaneous velocity of a $1 \mu \mathrm{m}$ diameter microsphere at $20{ }^{\circ} \mathrm{C}$. We need $G<2.5$ to measure the velocity of a $4.7 \mu \mathrm{m}$ diameter microsphere at the same conditions, and if the temperature is $60{ }^{\circ} \mathrm{C}$ the requirement is relaxed to $G<8.2$. This means that our detection system must be very close to the shot noise limit in order to measure the instantaneous velocity of a Brownian particle in water.

The discussions in the previous paragraphs assume that the normalized $\mathrm{VACF}$ is $\exp \left(-t / \tau_{p}^{*}\right)$ at short time scales. However, the normalized VACF is most likely $\exp \left(-b \sqrt{t / \tau_{f}}\right)$ at short time scales. This will require a temporal resolution of $\Delta t=\tau_{f} /\left(100 b^{2}\right)$ in order to measure the instantaneous velocity. Our optical tweezer will not be able to measure the instantaneous velocity of a Brownian particle in water if the normalized VACF follows $\exp \left(-b \sqrt{t / \tau_{f}}\right)$ even at such short time scales. On the other hand, the normalized VACF is much easier to measure than the instantaneous velocity. We should be able to measure the normalized VACF between 0.35 and 1 even if we are not able to measure the instantaneous velocity.

In the presence of detection noise, the measured position of the microsphere can be expressed as

$$
x_{m s r}(t)=x_{p}(t)+x_{n}(t),
$$


where $x_{p}(t)$ is the real position of the microsphere, and $x_{n}(t)$ is the noise of the detection system. The mean square displacement (MSD) of the measured positions is [91]:

$$
\begin{aligned}
M S D_{m s r}(t)= & \left\langle\left[x_{m s r}\left(t_{0}+t\right)-x_{m s r}\left(t_{0}\right)\right]^{2}\right\rangle \\
= & \left\langle\left[x_{p}\left(t_{0}+t\right)-x_{p}\left(t_{0}\right)\right]^{2}\right\rangle+\left\langle\left[x_{n}\left(t_{0}+t\right)-x_{n}\left(t_{0}\right)\right]^{2}\right\rangle \\
& +2\left\langle\left[x_{p}\left(t_{0}+t\right)-x_{p}\left(t_{0}\right)\right] \cdot\left[x_{n}\left(t_{0}+t\right)-x_{n}\left(t_{0}\right)\right]\right\rangle \\
= & M S D_{p}(t)+M S D_{n}(t) .
\end{aligned}
$$

The derivation assumes no correlation between the real position of the microsphere and the detection noise. In this case, the real MSD of the microsphere $\left(M S D_{p}(t)\right)$ can be obtained by subtracting the noise $M S D_{n}(t)$ from the measured $M S D_{m s r}(t)$, as is done in Ref. [29]. In reality, there may be a small correlation between the motion of the bead and the detection noise. Thus it is important to minimize the noise $M S D_{n}(t)$ in the measurement.

The measured velocity of the microsphere is

$$
\begin{aligned}
v_{m s r}(t) & =\frac{x_{m s r}\left(t+\frac{\delta t}{2}\right)-x_{m s r}\left(t-\frac{\delta t}{2}\right)}{\delta t} \\
& =\frac{x_{p}\left(t+\frac{\delta t}{2}\right)-x_{p}\left(t-\frac{\delta t}{2}\right)}{\delta t}+\frac{x_{n}\left(t+\frac{\delta t}{2}\right)-x_{n}\left(t-\frac{\delta t}{2}\right)}{\delta t} \\
& =v_{p}(t)+v_{n}(t),
\end{aligned}
$$

where $\Delta t \ll \tau_{p}$. Because the measured velocity contains a noise signal $v_{n}(t)$, the smallest possible $\delta t$ is not the optimal value for measuring the velocity. The DAQ card creates noise when it converts an analog signal to a digital signal due to the finite number of bits. The minimum value of $x_{n}\left(t+\frac{\delta t}{2}\right)-x_{n}\left(t-\frac{\delta t}{2}\right)$ 
is limited by the DAQ card, thus $v_{n}(t)$ may be larger than the real velocity of the microsphere $v_{p}(t)$ if $\delta t$ is too small.

The measured velocity represents the real instantaneous velocity of the microsphere if $v_{n}(t)$ is negligible. This requires $\left\langle v_{m s r}^{2}\right\rangle \gg\left\langle v_{n}^{2}\right\rangle$. One can check whether this condition is satisfied by comparing the signal when a microsphere is trapped in the optical tweezer and when there is not microsphere in the optical tweezer. The relation between $\left\langle v_{m s r}^{2}\right\rangle$ and $\left\langle v_{n}^{2}\right\rangle$ can also be obtained from the measured $M S D$ :

$$
\begin{aligned}
\left\langle v_{m s r}^{2}\right\rangle & =\left\langle\frac{\left[x_{m s r}\left(t+\frac{\delta t}{2}\right)-x_{m s r}\left(t-\frac{\delta t}{2}\right)\right]^{2}}{\delta t^{2}}\right\rangle \\
& =\frac{M S D_{m s r}(\delta t)}{\delta t^{2}} \\
& =\frac{M S D_{p}(\delta t)}{\delta t^{2}}+\frac{M S D_{n}(\delta t)}{\delta t^{2}} \\
& =\left\langle v_{p}^{2}\right\rangle+\left\langle v_{n}^{2}\right\rangle .
\end{aligned}
$$

Thus $\left\langle v_{m s r}^{2}\right\rangle \gg\left\langle v_{n}^{2}\right\rangle$ is equivalent to $M S D_{m s r}(\delta t) \gg M S D_{n}(\delta t)$.

The measured velocity autocorrelation function is

$$
\left\langle v_{m s r}\left(t+t_{0}\right) v_{m s r}\left(t_{0}\right)\right\rangle=\left\langle v_{p}\left(t+t_{0}\right) v_{p}\left(t_{0}\right)\right\rangle+\left\langle v_{n}\left(t+t_{0}\right) v_{n}\left(t_{0}\right)\right\rangle
$$

Since the noise of the detection system has almost no correlation, the last term of this equation can be neglected. Thus

$$
\left\langle v_{m s r}\left(t+t_{0}\right) v_{m s r}\left(t_{0}\right)\right\rangle \doteq\left\langle v_{n}\left(t+t_{0}\right) v_{n}\left(t_{0}\right)\right\rangle .
$$

So the measurement of the velocity autocorrelation function is not sensitive to the noise of the detection system. On the other hand, the measurement of the instantaneous velocity is very sensitive to the noise of the detection system. 
If the detection system samples the position of the microsphere every $d t$ that is much shorter than the required temporal resolution $\Delta t$, we can reduce the noise in the measured velocity by using successively averaged positions to calculate the velocity. Let $\delta t=N d t\left(N \ll \tau_{p} / d t\right)$, then

$$
x_{a v r}(t)=\frac{1}{N} \sum_{j=1}^{N} x_{m s r}\left(t+j d t-(N+1) \frac{d t}{2}\right) .
$$

The measured velocity becomes

$$
v_{m s r}(t)=\frac{x_{a v r}\left(t+\frac{\delta t}{2}\right)-x_{a v r}\left(t-\frac{\delta t}{2}\right)}{\delta t} .
$$

Then the velocity noise is

$$
\begin{aligned}
v_{n}(t)= & \frac{1}{N^{2} d t}\left[\sum_{j=1}^{N} x_{n}\left(t+j d t-(N+1) \frac{d t}{2}+\frac{\delta t}{2}\right)\right. \\
& \left.-\sum_{j=1}^{N} x_{n}\left(t+j d t-(N+1) \frac{d t}{2}-\frac{\delta t}{2}\right)\right] .
\end{aligned}
$$

On average, the rms amplitude of $v_{n}(t)$ is $N \sqrt{N}$ times smaller than that of $\left[x_{n}(t+d t / 2)-x_{n}(t-d t / 2)\right] / d t$ if the position noise $x_{n}(t)$ is white noise.

\subsection{A simple optical tweezer in water}

Figure 5.5 shows a simplified schematic of an optical tweezer for trapping a microsphere in water and studying its Brownian motion with ultrahigh resolution. The trapping laser passes through a $\lambda / 2$ waveplate and a polarizing beam splitter cube (PBS1), which control the power of the laser. The laser is then reflected by a dichroic mirror (DM2) and enters an objective lens 
(OB2) to form an optical tweezer inside a sample chamber at the focus of the objective lens. We use another objective lens (OB1) to collect the laser for detection. The sample chamber and the objective OB1 are both mounted on 3axis translation stages. The motion of a trapped microsphere causes deflection of the trapping laser. Thus we can monitor the position of the microsphere

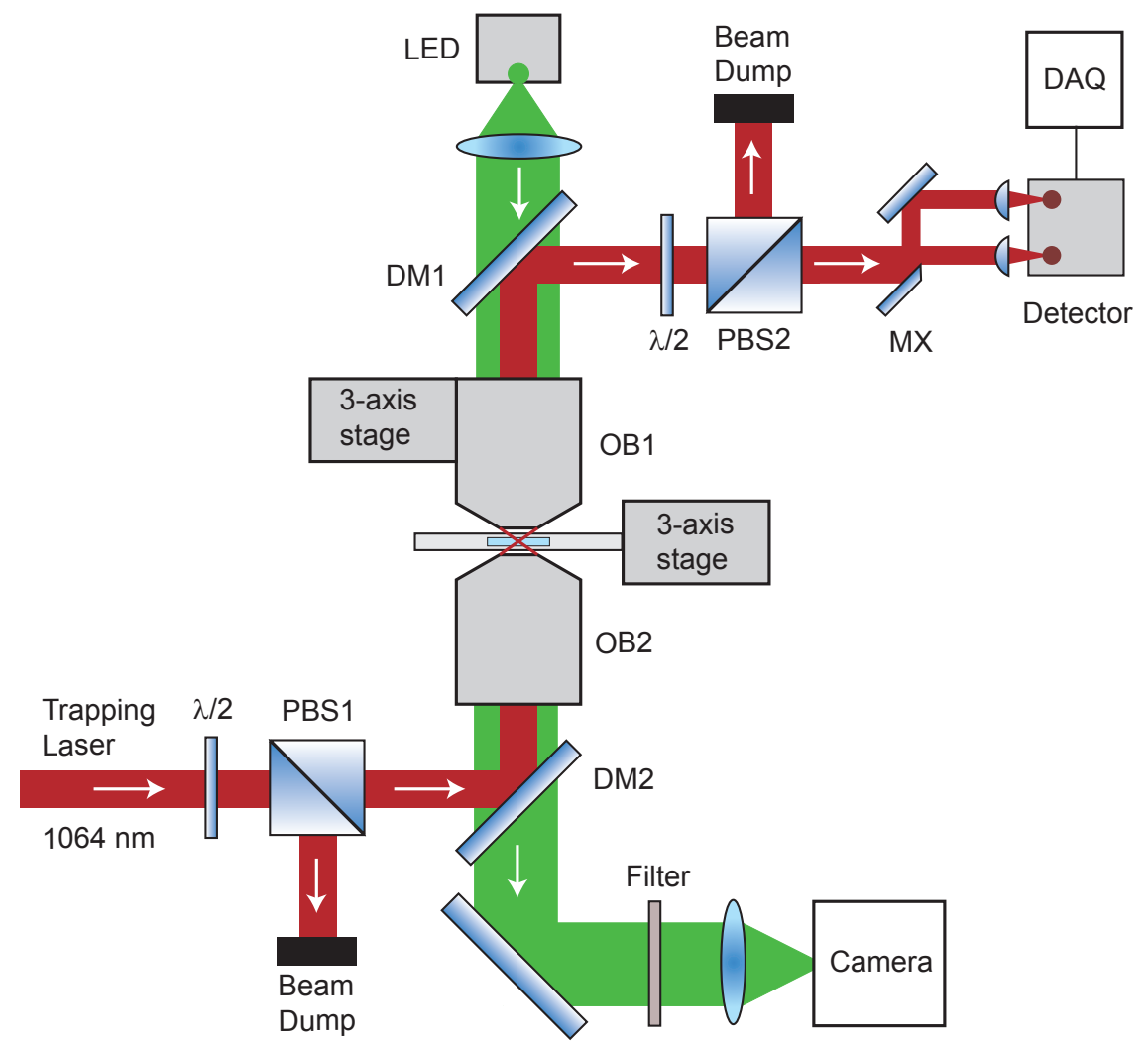

Figure 5.5: Simplified schematic of an optical setup for trapping microspheres in water. PBS1 and PBS2 are polarizing beam splitter cubes, DM1 and DM2 are dichroic mirrors, OB1 and OB2 are 100x oil-immersion objective lenses, MX is a mirror with a sharp edge that splits the beam into two parts horizontally, and DAQ is a data acquisition card that is installed in a computer. 
by measuring the deflection of the laser beam. After passing through another $\lambda / 2$ waveplate and beam splitter (PBS2) to reduce its power, the laser is split by a mirror with a sharp edge. The difference between the two halves is measured by a fast balanced detector $[28,33]$. The output signal of the detector is proportional to the position of a trapped microsphere, and is collected by a fast data acquisition card (DAQ). We use a white LED for illumination to take bright field images by a camera for real-time imaging of the microspheres. The filter before the camera blocks the scattered light from the trapping laser.

The trapping laser is an ultrastable NPRO laser (Model: 126-1063700, Lightwave Electronics (now JDSU)) with a wavelength of $1064 \mathrm{~nm}$. The maximum power is about $800 \mathrm{~mW}$. Its rms intensity noise is $<0.05 \%$ over the range from $10 \mathrm{~Hz}$ to $2 \mathrm{MHz}$, and is shot noise limited above $10 \mathrm{MHz}$. PBS1 is a high-energy laser-line polarizing cube beamsplitter from CVI Melles Griot (PBSO-1064-50, CW damage threshold: $1 \mathrm{MW} / \mathrm{cm}^{2}$ ). Both OB1 and OB2 are 100x oil-immersion objective lenses with NA $=1.25$. They are infiniteconjuncted objective lenses, and are designed for working with cover glasses with thickness of $0.17 \mathrm{~mm}$. OB1 and OB2 are bought from the Microscope Store, LLC (www.microscope.com). Their working distance is about $0.1 \mathrm{~mm}$. Thus the total distance between the front surfaces of the two objective lenses is about $0.54 \mathrm{~mm}$. Two oil layers, two cover glasses, two epoxy layers and one water layer must be made to fit within this gap. We later use an objective lens with a longer working distance to make the alignment easier.

The sample chamber consists of three layers of coverslips bonded to- 
gether by epoxy. The two outer layers are No. 0 coverslips (thickness: 0.08 $0.13 \mathrm{~mm}$ ), the center layer is a $1 \mathrm{~cm}^{2}$ square of water layer surrounded by four small pieces of No. 1 coverslips (thickness: $0.13-0.16 \mathrm{~mm}$ ). The coverslips are bought from Ted Pella, Inc. The microspheres are contained in the water
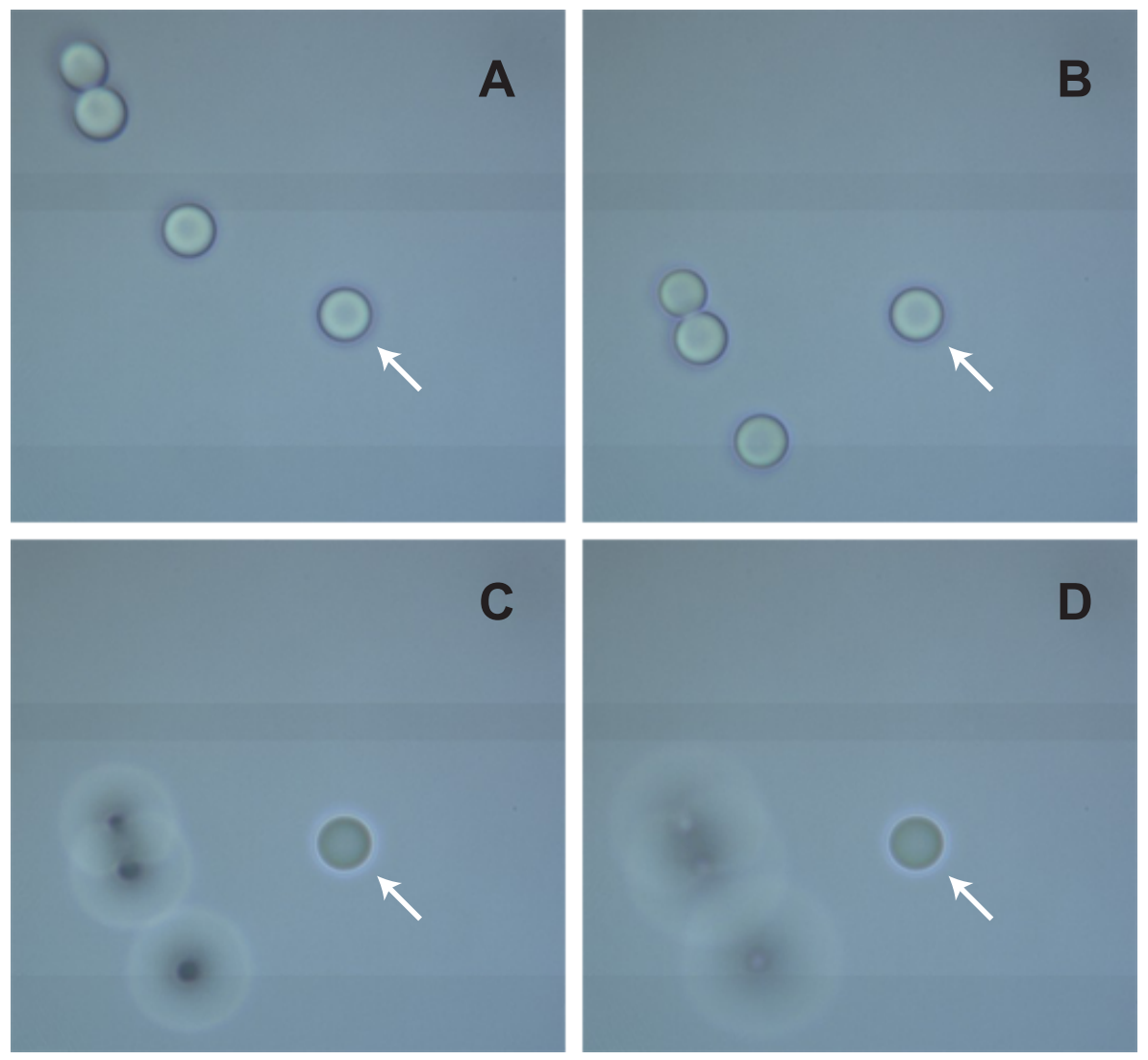

Figure 5.6: A 4.7- $\mu \mathrm{m}$ diameter silica microsphere (marked by an arrow) is trapped in water with an optical tweezer. The trapped microsphere does not move while other three microspheres are shifted when we move the glass slide perpendicular to the laser beam ( $\mathbf{A}$ and $\mathbf{B}$ ) or along the propagation direction of the laser beam ( $\mathbf{B}, \mathbf{C}$ and $\mathbf{D})$. In $\mathbf{C}$ and $\mathbf{D}$, the images of un-trapped microspheres become blurred as they are moved out of focus, while the images of the trapped microsphere are always sharp as it is trapped at the focus. 
layer. We use vacuum grease to seal the sample chamber. The sealed sample can be used for several hours. Otherwise the water will evaporate in about 10 minutes without sealing. The silica microspheres used for trapping are bought from Bangs Laboratories, Inc.

The mirror with a sharp edge (MX) is a D-Shaped mirror bought from Thorlabs, Inc. The detector is a balanced InGaAs photo detector. The two photodiodes are matched to cancel the common noise of the laser. The bandwidth of the detector is $100 \mathrm{MHz}$, enabling us to study the Brownian motion of a trapped microsphere at fast time scales. The DAQ card (Gage, Razor CompuScope 1622) has two channels with sampling rate of $200 \mathrm{MS} / \mathrm{s}$ and vertical resolution of 16 bits. This is one of the fastest high resolution digitizers currently available on the market. Our detection system can monitor the position of a trapped microshpere with sub-Ångstrom spatial resolution and sub-microsecond temporal resolution.

The maximum power of the LED is about $5 \mathrm{~W}$. The camera is a USB color 3MP CMOS camera bought from Mightex Systems (Model: MCE-C030U). It allows us to monitor the sample chamber in real time with spatial resolution of about $0.5 \mu \mathrm{m}$.

Figure 5.6 shows successful trapping of a $4.7-\mu \mathrm{m}$ diameter silica microsphere in water with an optical tweezer. Initially some microspheres undergo Brownian motion near the bottom of the sample chamber, while other microspheres are stuck to the surface of the coverslip due to the van der Waals force. We can use the optical tweezer to trap a microsphere that is not stuck 
on the surface. After trapping, the position of the trapped microsphere is fixed by the optical tweezer. We can move the glass slide in 3D while keeping the microsphere trapped, as shown in figure 5.6. An optical tweezer thus provides

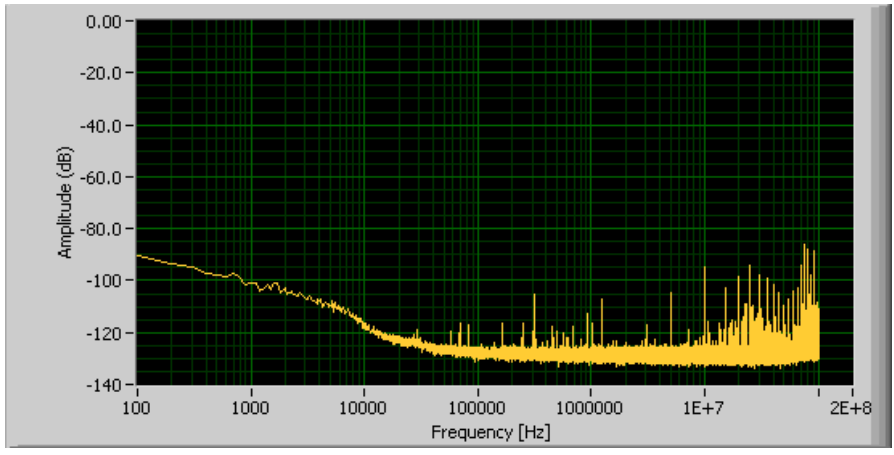

(A)

noise of

DAQ card only

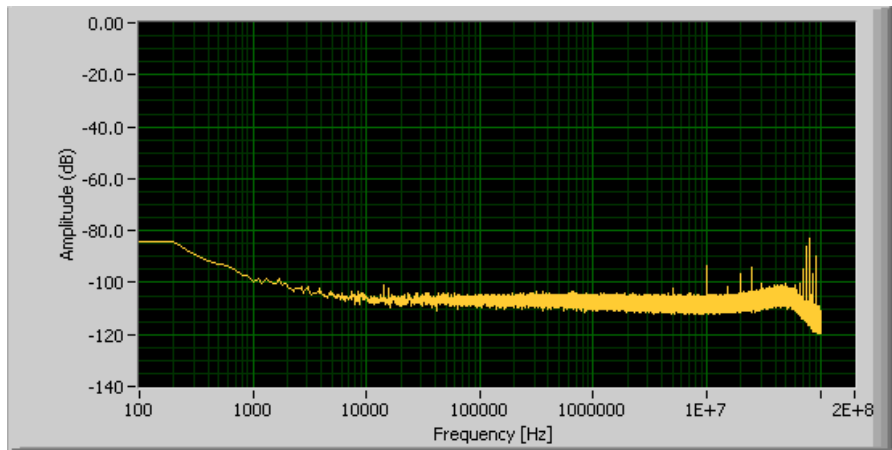

(B)

PDB120C

detector

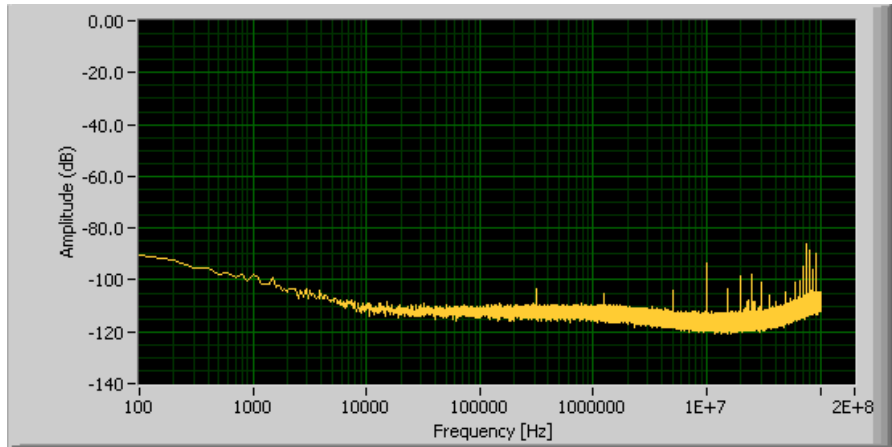

(C)

PDB110C-

$\mathrm{AC}$

detector

Figure 5.7: Noise spectra of the DAQ card and two different detectors. 
a tool to manipulate a microscopic object in $3 \mathrm{D}$ without physical contact.

Figure 5.7 shows the measured noise spectra of the digitizer and two balanced detectors(Thorlabs PDB120C, and PDB110C-AC). When no detector is connected to the DAQ card, the average noise spectral density is about $-130 \mathrm{~dB}$ around $10 \mathrm{MHz}$. When a PDB120C detector is connected to the DAQ card, the average spectral density increases to about $-107 \mathrm{~dB}$ around $10 \mathrm{MHz}$. When a PDB110C-AC detector is connected to the DAQ card, the
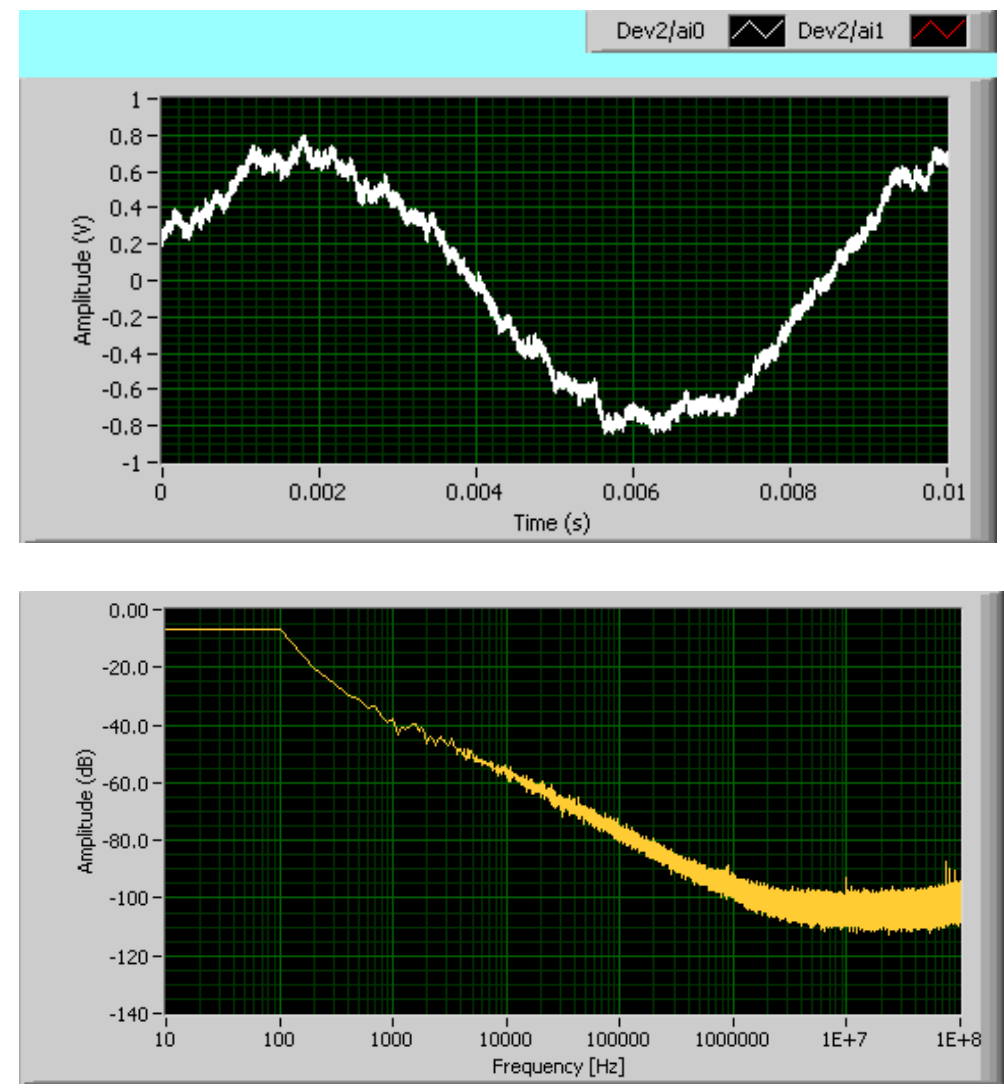

Figure 5.8: A waveform and spectrum of a 4.7- $\mu \mathrm{m}$ diameter microsphere trapped in water at room temperature. 
average spectral density increases to about $-115 \mathrm{~dB}$ around $10 \mathrm{MHz}$. Thus the PDB110C-AC detector has less noise than the PDB120C detector. So we use the PDB110C-AC detector for studying the Brownian motion in water.

Fig. 5.8 shows a measured waveform and power spectrum of a $4.7-\mu \mathrm{m}$ diameter microsphere trapped in water at room temperature. The waveform appears as a sine wave at $120 \mathrm{~Hz}$. We found that it was due to mechanical
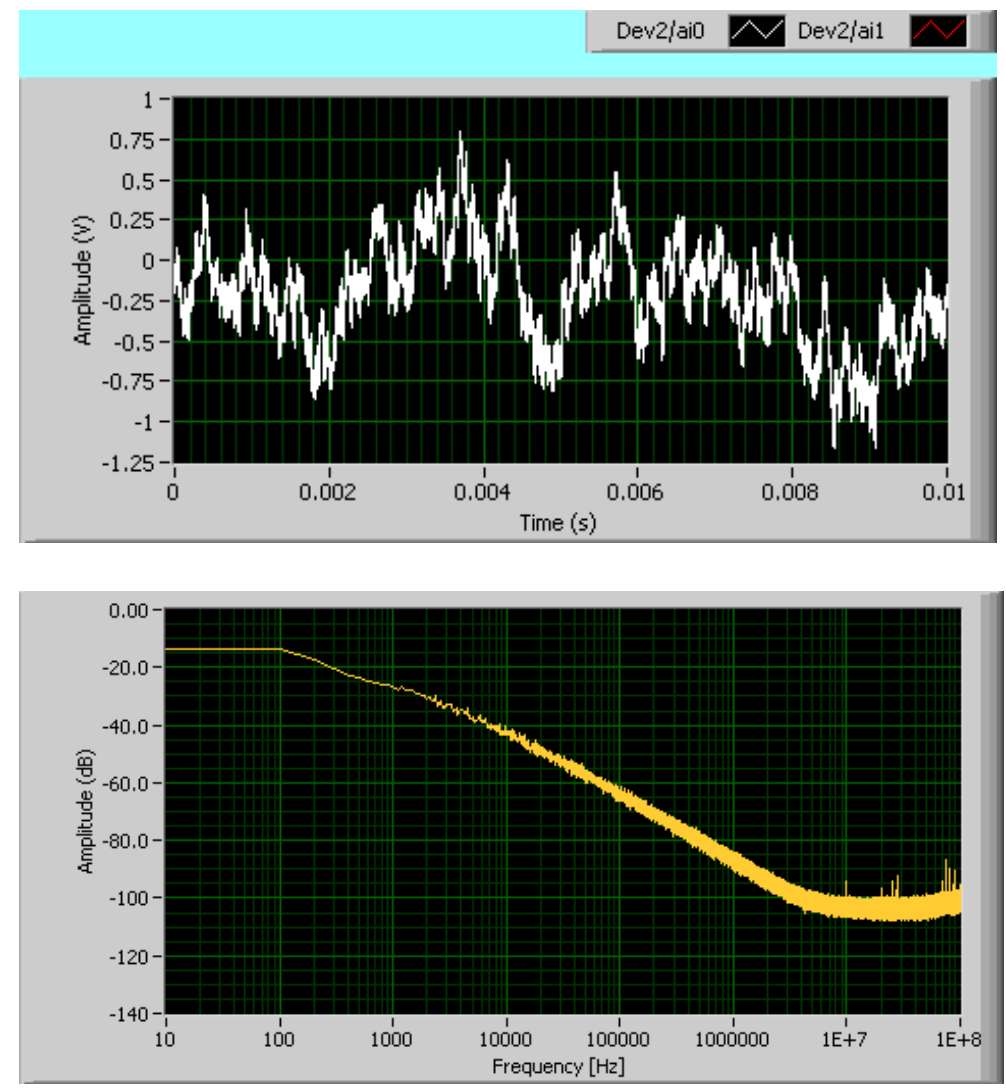

Figure 5.9: A waveform and spectrum of a 4.7- $\mu \mathrm{m}$ diameter microsphere trapped in water at room temperature. A copper block was mounted on the post of the objective lens to reduce mechanical vibration. 
vibration of the objective lens. The real signal of the Brownian motion of the microsphere is the high frequency fluctuations in the "sine" curve. As a result, the power spectrum is very small at frequencies above $1 \mathrm{MHz}$. The power spectrum at frequencies above $1 \mathrm{MHz}$ basically comes from the noise of the laser, which is a problem for studying the Brownian motion of the microsphere.

Later we mounted a heavy copper block on the post of the top objective lens (OB1 in Fig. 5.5) to reduce the mechanical vibration. This improves the signal significantly. Fig. 5.9 shows a measured waveform and power spectrum of a $4.7-\mu \mathrm{m}$ diameter microsphere trapped in water after we mounted a copper block on the post. It no longer appears like a sine wave. The high frequency

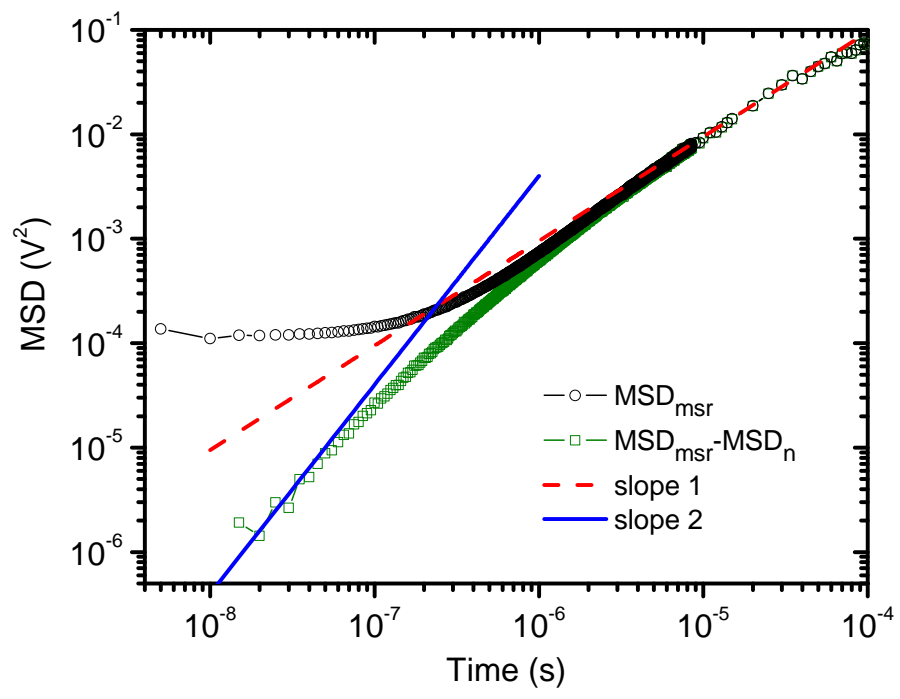

Figure 5.10: Preliminary results of the MSD of a $4.7-\mu \mathrm{m}$ bead in water. 
fluctuation due to the Brownian motion of the microsphere dominates the signal. As a result, the power spectral density increased by about $7 \mathrm{~dB}$ at 1 MHz. The signal is about $-95 \mathrm{~dB}$ at $2 \mathrm{MHz}$, and the noise of the detection system is about $-103 \mathrm{~dB}$. So the signal is only $8 \mathrm{~dB}$ above the detection noise at $2 \mathrm{MHz}$. This is not enough for measuring the instantaneous velocity of the Brownian motion. The signal needs to be increased by $10 \mathrm{~dB}$ or more in order to measure the instantaneous velocity of the $4.7-\mu \mathrm{m}$ diameter microsphere in water.

Fig. 5.10 show preliminary results of the MSD of a $4.7-\mu \mathrm{m}$ bead in water. The measured $M S D_{m s r}(t)$ is dominated by the noise when the time is shorter than $0.1 \mu \mathrm{s}$. The $M S D_{p}(t)=M S D_{m s r}(t)-M S D_{n}(t)$ agrees with the prediction of slope 2 at short time scales. However, this subtraction method is limited by the uncertainty on determining $M S D_{n}(t)$.

\subsection{Interferometer-enhanced optical tweezers}

In order to measure the instantaneous velocity of a $4.7 \mu \mathrm{m}$ microsphere in water, we need to improve the signal-to-noise ratio of the detection system by a factor of 10 or more at frequencies above $1 \mathrm{MHz}$.

We consider the detection of the position of a trapped microsphere along $x$ axis that is perpendicular to the propagating direction of the laser. Suppose the position of the microsphere is $x_{p}$, and the electric field of the optical tweezer

without the microsphere is $E_{0}(x)\left(1+\varepsilon_{\text {int }}+\varepsilon_{p t}(x)\right)$, where $\varepsilon_{\text {int }}$ represents the intensity fluctuation of the laser, and $\varepsilon_{p t}(x)$ represents the pointing fluctuation 
of the laser. The electric field with a trapped microsphere can be written as:

$$
E_{p}(x)=E_{0}(x)\left(1+\varepsilon_{i n t}+\varepsilon_{p t}(x)\right)\left(1+\xi\left(x_{p}\right)\right)
$$

where $\xi\left(x_{p}\right)$ represents the effect of the microsphere. If we assume $\varepsilon_{i n t} \ll 1$, $\varepsilon_{p t} \ll 1$ and $\xi\left(x_{p}\right) \ll 1$, the intensity of the laser beam after the microsphere is

$$
I_{p}(x) \doteq I_{0}(x)\left(1+2 \varepsilon_{\text {int }}+2 \varepsilon_{p t}(x)+2 \xi\left(x_{p}\right)\right)
$$

where $I_{0}(x)=E_{0}^{2}(x)$ is assumed to be a Gaussian function.

If we measure the position of the microsphere with a balanced detector (Fig. 5.5), we are measuring the difference of the power of the two halves of the laser beam:

$$
\Delta P\left(x_{p}, x_{m i r}\right)=\int_{x_{m i r}}^{+\infty} I_{p}(x) d x-\int_{-\infty}^{x_{m i r}} I_{p}(x) d x
$$

where $x_{m i r}$ is the position of the split mirror. Let us choose a $x_{m i r}$ to satisfy

$$
\int_{x_{m i r}}^{+\infty} I_{0}(x)\left(1+2 \varepsilon_{i n t}\right) d x-\int_{-\infty}^{x_{m i r}} I_{0}(x)\left(1+2 \varepsilon_{i n t}\right) d x=0
$$

then the effect of the intensity fluctuation can be eliminated. The output signal $\Delta P\left(x_{p}, x_{m i r}\right)$ contains the position signal of the microsphere and the pointing fluctuation of the laser beam.

If we use a beam splitter to split a laser to two beams, the profiles of the two beams are the same. We can use one beam to trap the microsphere, and the other beam as a reference. The electric field of the reference beam is

$$
E_{r e f}(x)=E_{0}(x)\left(1+\varepsilon_{i n t}+\varepsilon_{p t}(x)\right)
$$


If we add a phase delay to this reference beam, we can change its sign. Then we can combine the trapping beam and the reference beam together. The electric of the combined beam is

$$
E_{c b}(x)=E_{p}(x)-E_{r e f}(x)=E_{0}(x)\left(1+\varepsilon_{i n t}+\varepsilon_{p t}(x)\right) \xi\left(x_{p}\right)
$$

Since $\varepsilon_{\text {int }} \ll 1$ and $\varepsilon_{p t} \ll 1$, we have

$$
E_{c b}(x) \doteq E_{0}(x) \xi\left(x_{p}\right)
$$

The effects of the intensity fluctuation and the pointing fluctuation of the laser beam are almost eliminated from the combined beam. Thus the signalto-noise ratio can be increased significantly with an interferometer enhanced optical tweezer. The difficulty of this method is that the reference laser must have the same pointing fluctuation as the trapping beam at high frequencies $(>1 \mathrm{MHz})$.

Fig. 5.11 shows a Mach-Zehnder interferometer enhanced optical tweezer and detection system. The two objective lenses change the electric field from $E(x, y)$ to $E(-x,-y)$, so another pair of lenses is used to correct this. A mirror changes the electric field in one direction, i.e. from $E(x, y)$ to $E(-x, y)$. The trapping beam is reflected by three mirrors, and the reference beam is reflected by one mirror. So both beams are changed from $E(x, y)$ to $E(-x, y)$. We also need an EOM to change the phase of the reference beam. Because of low frequency mechanical vibration of mirrors, the phase of the reference beam must be actively locked to the trapping beam. A Sagnac interferometer 
enhanced optical tweezer may solve this problem [94], but its optics seem much more difficult to align.

We eventually decided to use a Wollaston interferometer enhanced optical tweezer to increase the signal-to-noise ratio. As shown in Fig. 5.12, its setup is very similar to a simple optical tweezer (Fig. 5.5 ). The Wollaston prism W2 (Karl Lambrecht Corp., Model: WQ-25-05-V 1064NM) separates the laser to two beams with different polarizations. The angle between the two beams is about $0.5^{\circ}$. These two beams form two optical tweezers that are separated by about $15 \mu \mathrm{m}$ at the focus of the objective lens. One optical tweezer traps a microsphere, and the other optical tweezer serves as a reference. These two beams are combined together by another Wollaston prism

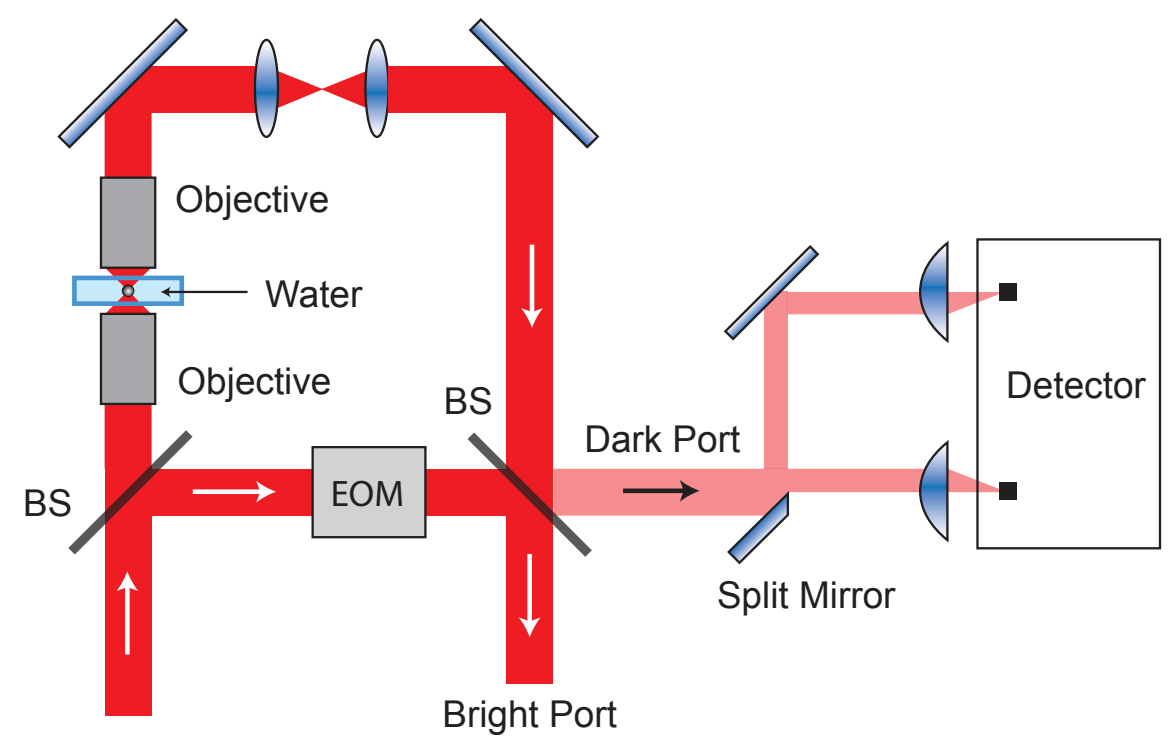

Figure 5.11: A Mach-Zehnder interferometer enhanced optical tweezer and detection system. 
[95]. We tune the angle of the $\lambda / 2$ waveplate before the PBS2 so that the two beams interfere destructively at one port of the PBS2. The split detection system is at the dark port. Compared with the Mach-Zehnder interferometer, the Wollaston interferometer is much easier to align. Both beams pass through the same optical path, so the reference and trapping beams contain the same pointing fluctuation. Thus the effect of the pointing fluctuation in detection can be eliminated.

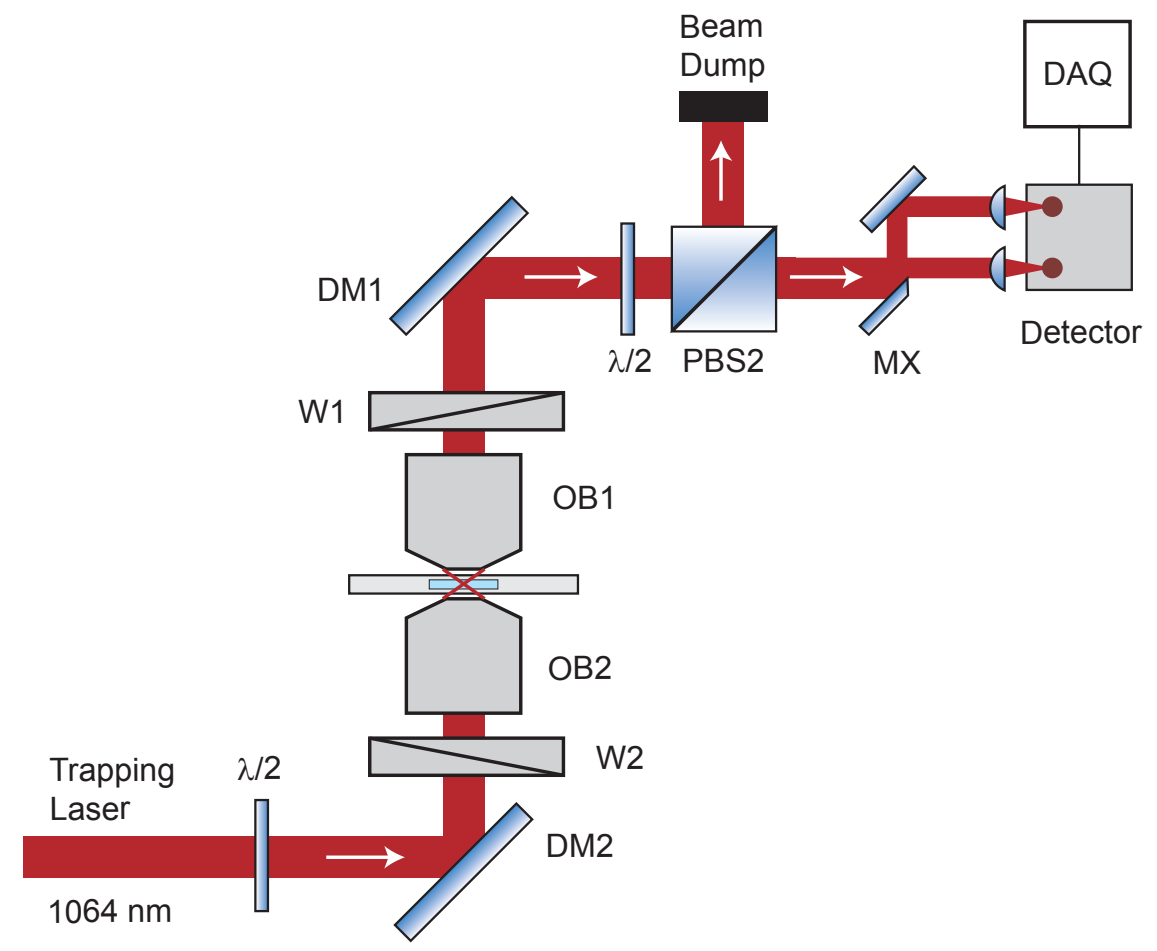

Figure 5.12: A Wollaston interferometer enhanced optical tweezer and detection system. The W1 and W2 are two Wollaston prisms. Other components are the same as in Fig. 5.5. 
We are currently working on this Wollaston interferometer enhanced optical tweezer. It should provide enough signal-to-noise ratio for measuring the normalized VACF of a Brownian particle in water between 0.35 and 1 . It will also have many applications in biophysics. 


\section{Chapter 6}

\section{Millikelvin cooling of an optically trapped microsphere in vacuum}

\subsection{Background}

Optical cooling and trapping of atoms [47-49] has led to dramatic breakthroughs in atomic, molecular and optical physics, including a new generation of atomic clocks, and realization of Bose-Einstein condensation and degenerate Fermi gas. Applying similar techniques to cool the mechanical motion of macroscopic objects towards the quantum ground state will benefit ultrahigh precision measurements and fundamental tests of macroscopic quantum physics $[7,9,13]$. A major obstacle to achieving ground-state cooling of most mechanical oscillators [83,96-101] is the thermal contact between oscillators and their environment. Recently, it was proposed that optical trapping of dielectric objects in vacuum would greatly reduce the thermal contact, and could even allow ground-state cooling from room temperature [17-19, 33, 102 106]. Besides providing ideal isolation from the environment, the optical trap can be switched off for time-of-flight measurements to perform full tomography of the mechanical state $[16,106]$. Here we report optical trapping of $\mathrm{SiO}_{2}$ microspheres in vacuum with high oscillation frequencies, and cooling of the center-of-mass motion to millikelvin temperatures with active feedback. 
Feedback control has been used widely in industry and scientific experiments. The most commonly used feedback controller is a proportionalintegral-derivative (PID) controller. A typical application of a PID controller is to stabilize the temperature of a system. In our lab, we routinely use PID controllers to stabilize the intensity of lasers.

A simple pendulum in air will oscillate if it is initially moved away from the equilibrium position. After oscillating for some time, the pendulum will decay to rest. In reality, however, the pendulum always vibrates with a small amplitude, due to external forces from seismic motion, and more fundamentally, due to the thermal Brownian stochastic force. Even at absolute zero temperature, the pendulum still oscillates because of the quantum zero-point energy. We can use feedback control to reduce the vibration amplitude. If the final vibration amplitude is smaller than the Brownian motion amplitude at thermal equilibrium, the feedback control is called "feedback cooling" unless the reduction in amplitude corresponds to an increase in frequency. In order to do feedback cooling, the detection system must at least be able to resolve the Brownian motion of the system at thermal equilibrium.

According to the equipartition theorem, the root mean square (rms) amplitude of the Brownian motion of a trapped microsphere at thermal equilibrium is $x_{r m s}=\sqrt{k_{B} T_{0} /\left(M \omega^{2}\right)}$, where $T_{0}$ is the environmental (air) temperature, $M$ is the mass of the microsphere, and $\omega$ is the angular trapping frequency. The characteristic size of the quantum ground-state wavefunction is $x_{\text {ground }}=\sqrt{\hbar /(M \omega)}$, where $\hbar$ is Planck's constant $/ 2 \pi$. 
Previous experiments have demonstrated optical levitation of a $20-\mu \mathrm{m}$ diameter sphere in vacuum with a trapping frequency of about $20 \mathrm{~Hz}$ [45], as well as feedback control of a trapped sphere which was used to increase the trapping frequency to several hundred hertz and stabilize its position to within a fraction of one micrometer [107]. However, the resolution of its detection system [107] was not sufficient to enable feedback cooling. For a $20-\mu \mathrm{m}$ diameter sphere trapped at $100 \mathrm{~Hz}$, the rms amplitude is about $0.04 \mu \mathrm{m}$ at $300 \mathrm{~K}$, and will be much smaller at lower temperature. The size of the quantum groundstate wavefunction is $x_{\text {ground }}=0.14 \mathrm{pm}$. Both values are far smaller than the resolution of the detection system of the previous experiment [107]. It is also important that the trapping frequency be much higher than the frequencies of seismic vibration in order to achieve significant cooling.

This chapter describes our efforts on feedback cooling of an optically trapped microsphere in vacuum. We use a dual-beam optical tweezer to trap a $3.0-\mu \mathrm{m}$ diameter sphere in vacuum with much higher oscillation frequencies (about $10 \mathrm{kHz}$ ) to minimize the effects of instrumental vibration. We also demonstrate a detection system to monitor the motion of a trapped microsphere with a sensitivity of about $39 \mathrm{fm} / \sqrt{\mathrm{Hz}}$ over a wide frequency range. Using active feedback, we simultaneously cool the three center-of-mass (COM) vibration modes of a microsphere from room temperature to a minimum mode temperature of $1.5 \mathrm{mK}$, which corresponds to the reduction of the rms amplitude of the microsphere from $6.7 \mathrm{~nm}$ to $15 \mathrm{pm}$ for that mode. 


\subsection{Principle of feedback cooling}

An optically trapped microsphere in non-perfect vacuum will exhibit Brownian motion due to collisions between the microsphere and residual air molecules. When there is no feedback cooling, the equation of the Brownian motion of an optically trapped microsphere is:

$$
\frac{d^{2} x_{j}}{d t^{2}}+\Gamma_{0} \frac{d x_{j}}{d t}+\Omega_{j}^{2} x=F_{j}^{t h}
$$

where $\Gamma_{0}$ is the viscous damping factor due to air molecules, $\Omega_{j} / 2 \pi(\mathrm{j}=1,2,3)$ are the resonant frequencies of the optical trap along the three fundamental axes (x, y, and z axes), and $F_{j}^{t h}=\zeta_{j}(t) \sqrt{2 k_{B} T \Gamma_{0} / M}$ is the Brownian stochastic force.

The damping term $\Gamma_{0} \frac{d x}{d t}$ tends to stop any vibration, while the $F_{j}^{t h}$ term drives the motion. It is very interesting that $\Gamma_{0}$ is also contained in $F_{j}^{t h}$. When the mechanical energy (sum of the kinetic energy and the potential energy) of the microsphere is larger than $k_{B} T$ in one direction, the $\Gamma_{0} \frac{d x}{d t}$ term will dominate and the mechanical energy of the microsphere will be reduced. On the other hand, the $F_{j}^{t h}$ term will dominate and increase the mechanical energy of the microsphere if its mechanical energy is smaller than $k_{B} T$. Thus the average mechanical energy of the microsphere will be $k_{B} T$ in each direction at thermal equilibrium.

At thermal equilibrium, the power spectrum of COM motion of a trapped microsphere along each of the three fundamental mode axes is $[83,96]$ :

$$
S_{j}(\omega)=\frac{2 k_{B} T_{0}}{M} \frac{\Gamma_{0}}{\left(\Omega_{j}^{2}-\omega^{2}\right)^{2}+\omega^{2} \Gamma_{0}^{2}},
$$


where $\omega / 2 \pi$ is the observation frequency.

\subsubsection{Feedback cooling}

To implement feedback cooling, we apply an external force on the trapped microsphere:

$$
F_{j}^{c o o l}=-\Gamma_{j}^{c o o l} \frac{d x_{j}}{d t}
$$

The force is proportional to the velocity of the microsphere but with opposite direction. Thus it will slow down the motion of the microsphere. With feedback cooling, the equation of the Brownian motion of an optically trapped microsphere is:

$$
\frac{d^{2} x_{j}}{d t^{2}}+\left(\Gamma_{0}+\Gamma_{j}^{c o o l}\right) \frac{d x_{j}}{d t}+\Omega_{j}^{2} x=\zeta_{j}(t) \sqrt{\frac{2 k_{B} T \Gamma_{0}}{M}} .
$$

In contrast to the $\Gamma_{0}$ due to air molecules, $\Gamma_{j}^{c o o l}$ is only contained in the damping term but not in the heating term. So "feedback cooling" is also called "cold damping".

With feedback cooling, the power spectrum of the COM motion of a trapped microsphere along each of the three fundamental mode axes is:

$$
S_{j}^{c o o l}(\omega)=\frac{2 k_{B} T_{0}}{M} \frac{\Gamma_{0}}{\left(\Omega_{j}^{2}-\omega^{2}\right)^{2}+\omega^{2}\left(\Gamma_{0}+\Gamma_{j}^{c o o l}\right)^{2}} .
$$

Let $\Gamma_{j}^{t o t}=\Gamma_{0}+\Gamma_{j}^{c o o l}$ be the total damping factor, and $T_{j}^{c o o l}=T_{0} \Gamma_{0} / \Gamma_{j}^{t o t}$ be the effective temperature of the motion with feedback cooling, the power spectrum can be rewritten as:

$$
S_{j}^{c o o l}(\omega)=\frac{2 k_{B} T_{j}^{c o o l}}{M} \frac{\Gamma_{j}^{t o t}}{\left(\Omega_{j}^{2}-\omega^{2}\right)^{2}+\omega^{2}\left(\Gamma_{j}^{t o t}\right)^{2}},
$$


which has the same form as Eq. 6.2. The effective temperature along each axis

may be different because $\Gamma_{j}^{c o o l}$ can be different along different directions. The motion can be cooled significantly by applying a feedback damping $\Gamma_{j}^{c o o l}>>$ $\Gamma_{0}$. The lowest temperature will be limited by the noise in the detection system and feedback circuits, as well as coupling between different directions.

\subsubsection{Feedback amplification}

Besides cooling, feedback control can also be used to amplify the motion. We can apply a force in the same direction as the velocity of the microsphere to amplify the motion:

$$
F_{j}^{a m p}=+\Gamma_{j}^{a m p} \frac{d x_{j}}{d t} .
$$

With feedback amplification, the equation of the Brownian motion of an optically trapped microsphere is:

$$
\frac{d^{2} x_{j}}{d t^{2}}+\left(\Gamma_{0}-\Gamma_{j}^{a m p}\right) \frac{d x_{j}}{d t}+\Omega_{j}^{2} x=\zeta_{j}(t) \sqrt{\frac{2 k_{B} T \Gamma_{0}}{M}} .
$$

The power spectrum of COM motion of the microsphere is:

$$
S_{j}^{a m p}(\omega)=\frac{2 k_{B} T_{0}}{M} \frac{\Gamma_{0}}{\left(\Omega_{j}^{2}-\omega^{2}\right)^{2}+\omega^{2}\left(\Gamma_{0}-\Gamma_{j}^{a m p}\right)^{2}} .
$$

When $\Gamma_{j}^{a m p}<\Gamma_{0}$, the system is stable. The effect of $\Gamma_{j}^{a m p}$ is to amplify the motion and decrease the linewidth of the vibration from $\Gamma_{0}$ to $\Gamma_{0}-\Gamma_{j}^{a m p}$. When $\Gamma_{j}^{a m p}>\Gamma_{0}$, the system is not stable and the microsphere will be lost. We have observed both feedback cooling and feedback amplification in our experiment by inverting the velocity signal. 


\subsubsection{Heating due to light scattering}

For a microsphere trapped by an optical tweezer, there are also heating effects due to the light scattered by the microsphere. The heating effects of light scattering can be separated to two parts. The first part is because of the shot noise of the laser. This effect is very small and has been calculated in Refs. [17, 18].

The second part is because the scattering force is not conservative. The scattering force can do net work on the microsphere when the microsphere moves over a closed loop under certain conditions [67]. If the two counterpropagating beams of our dual-beam optical trap is slightly misaligned along $x$ axis (Fig. 3.10), the scattering force on the microsphere is along the axial direction ( $z$ axis), and is proportional to the displacement of the microsphere

along $x$ axis. We have $\vec{F}_{\text {scat }} \propto x \hat{z}$. For a microsphere moving in a loop in the $x-z$ plane, the net work done by the scattering force over a loop is

$$
W=\oint \vec{F}_{\text {scat }} d l \propto \sqrt{\left\langle x^{2}\right\rangle} \sqrt{\left\langle z^{2}\right\rangle}
$$

The period of a harmonic oscillator is independent of the energy of the oscillator. The mechanical energy of the microsphere will increase due to the work done by the scattering force at a rate:

$$
\frac{d}{d t}\left(E_{x}+E_{z}\right) \propto \sqrt{\left\langle x^{2}\right\rangle} \sqrt{\left\langle z^{2}\right\rangle} \propto \sqrt{E_{x} E_{z}}
$$

where $E_{x}$ and $E_{z}$ are mechanical energies of the microsphere. From this equation, it is clear that the scattering force couples the motion along different 
directions and can make the dynamics of the system very complex. Here we will try to obtain some qualitative properties of this heating effect. For simplicity, we assume that $E_{x}$ is proportional to $E_{z}$, then $d E_{x} / d t \propto E_{x}$. So the scattering force will cause a heating that is proportional to the mechanical energy of the microsphere. This effect can be minimized by better alignment of the two counter-propagating laser beams.

A phenomenological description of the feedback cooling process with this heating effect can be written in an equation about the average mechanical energy of the microsphere along each axis:

$$
\frac{d E_{j}}{d t}=-\Gamma_{0} E_{j}-\Gamma_{j}^{c o o l} E_{j}+\Gamma_{0}\left(k_{B} T\right)+\alpha_{j} E_{j}
$$

The first term $\left(-\Gamma_{0} E_{j}\right)$ describes the damping due to the air, the second term is due to feedback cooling, the third term describes the heating effect due to the air, and the last term describes the heating effect due to the scattering force. The final effective temperature of motion of the microsphere in each direction is

$$
T_{j}^{c o o l}=E_{j} / k_{B}=\frac{\Gamma_{0} T}{\Gamma_{0}+\Gamma_{j}^{c o o l}-\alpha_{j}} .
$$

The system will be stable when $\Gamma_{0}+\Gamma_{j}^{c o o l}>\alpha_{j}$ and unstable when $\Gamma_{0}+\Gamma_{j}^{c o o l}<$ $\alpha_{j}$. This provides a method to estimate $\alpha_{j}$ experimentally. To estimate the $\alpha_{j}$, we turn off the feedback cooling forces to let $\Gamma_{j}^{c o o l}=0$. We first trap a microsphere at a high pressure. At high pressures, we have $\Gamma_{0}>\alpha_{j}$, and the system is stable. We then reduce the air pressure to reduce $\Gamma_{0}$. At a certain pressure, the system becomes unstable and microsphere is lost. Then 
we have $\alpha_{j} \approx \Gamma_{0}^{e s c}$, where $\Gamma_{0}^{e s c}$ is the air damping factor at the moment when the microsphere is lost.

The heating rate due to the nonconservative force is proportional to the energy of the microsphere. As the energy of the microsphere is reduced by cooling, this heating effect becomes negligible and will not prevent ground state cooling. For cooling, we usually have $\Gamma_{j}^{c o o l} \gg \alpha_{j}$, thus the effect of $\alpha_{j}$ on the final temperature is very small (Eq. 6.13). The heating effect of the scattering force will be much smaller for a single-beam optical tweezer.

The above discussions are based on classical mechanics, which is sufficient for understanding our current experiment. Quantum mechanical description of feedback cooling will be necessary if the motion of the microsphere is cooled to near the quantum ground state $[82,108,109]$.

\subsubsection{Damping due to the residual gas in vacuum}

The viscous damping factor due to air can be calculated by kinetic theory. Assuming the reflection of air molecules from the surface of a microsphere is diffusive, and the molecules thermalize with the surface during collisions, we have $[110]$

$$
\Gamma_{0}=\frac{6 \pi \eta R}{M} \frac{0.619}{0.619+K n}\left(1+c_{K}\right)
$$

where $\eta$ is the viscosity coefficient of the air, $R$ is the radius of the microsphere, $M$ is the mass of the microsphere, and $K n=l / R$ is the Knudsen number. Here $l$ is the mean free path of the air molecules. $c_{K}=(0.31 K n) /(0.785+$ $\left.1.152 K n+K n^{2}\right)$ is a small positive function of $K n$ [110]. At low pressures 
where $K n \gg 1$, the viscous damping factor is proportional to the pressure. At high pressures where $K n \ll 1$, the viscous damping factor is $\Gamma_{0}=6 \pi \eta R / M$, which is the same as the prediction of Stoke's law.

Figure 6.1 shows the measured linewidth, $\Gamma_{0} / 2 \pi$, of the oscillation of

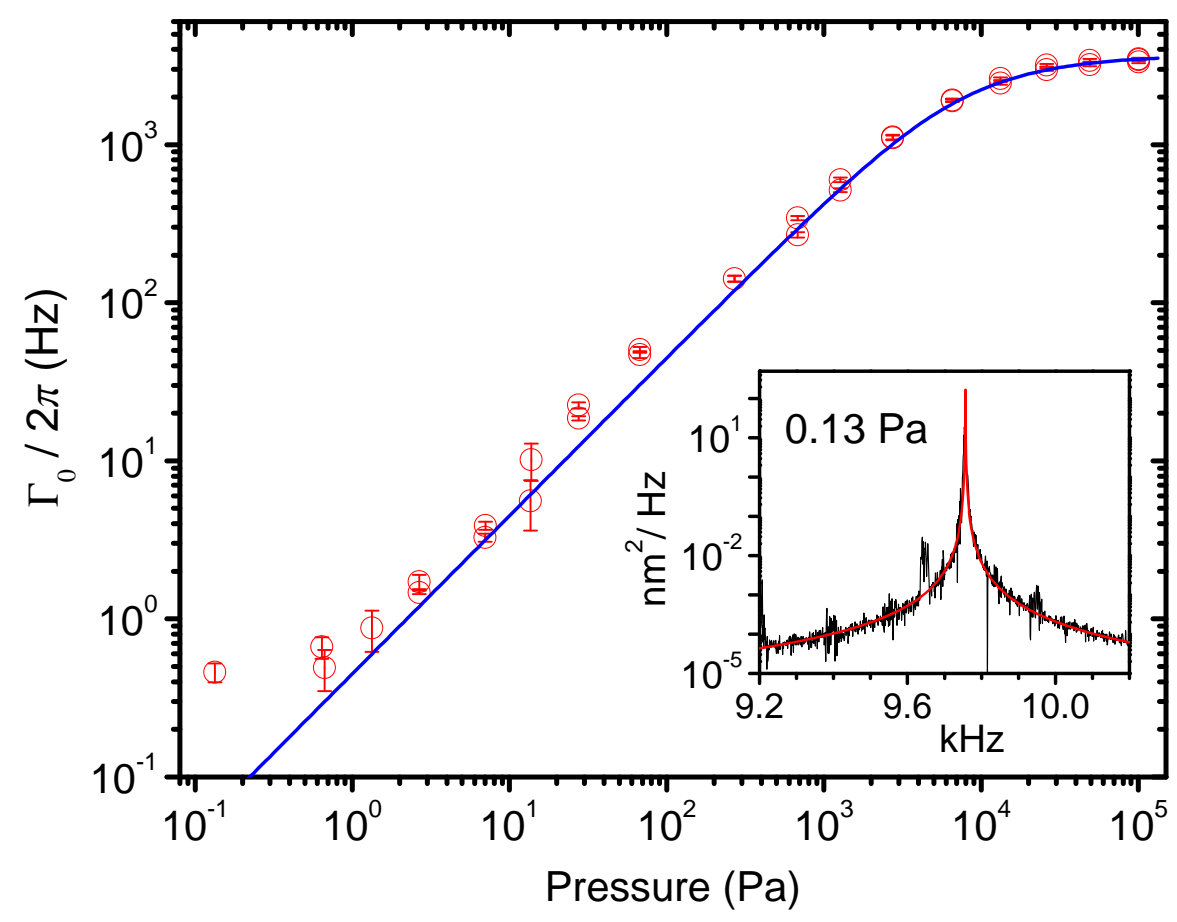

Figure 6.1: Measured linewidths of the oscillation of an optically trapped 3- $\mu \mathrm{m}$ diameter microsphere at different pressures. The blue curve is the prediction of a kinetic theory (Eq. 6.14). The inset is the measured power spectrum at $0.13 \mathrm{~Pa}$. By fitting the spectrum with Eq. 6.2 (red curve), we obtain $\omega_{1}=2 \pi \cdot(9756.4 \pm 0.3) \mathrm{Hz}$ and $\Gamma_{0}=2 \pi \cdot(0.46 \pm 0.06) \mathrm{Hz}$ for this example. The same method is used to obtain linewidths for other pressures. 
a trapped $3-\mu \mathrm{m}$ microsphere at different pressures without feedback cooling. The powers of the two trapping beams are $120 \mathrm{~mW}$ and $100 \mathrm{~mW}$, respectively. The linewidths are obtained by fitting the measured power spectra with Eq. 6.2. The measured linewidths agree very well with the prediction of kinetic theory (Eq. 6.14) from $10^{5} \mathrm{~Pa}$ down to $1 \mathrm{~Pa}$. At pressures below $1 \mathrm{~Pa}$, the measured linewidths are larger than the theoretical prediction. This linewidth broadening is due to power fluctuations of the trapping laser. The inset of Fig. 6.1 shows a power spectrum at $0.13 \mathrm{~Pa}$. A more detailed power spectrum at $0.13 \mathrm{~Pa}$ ( 1 mtorr) is shown in Fig. 6.2. The trapping frequency $\omega_{1} / 2 \pi$ is $9756.4 \pm 0.3 \mathrm{~Hz}$, and the linewidth is $0.46 \pm 0.06 \mathrm{~Hz}$, giving a quality factor

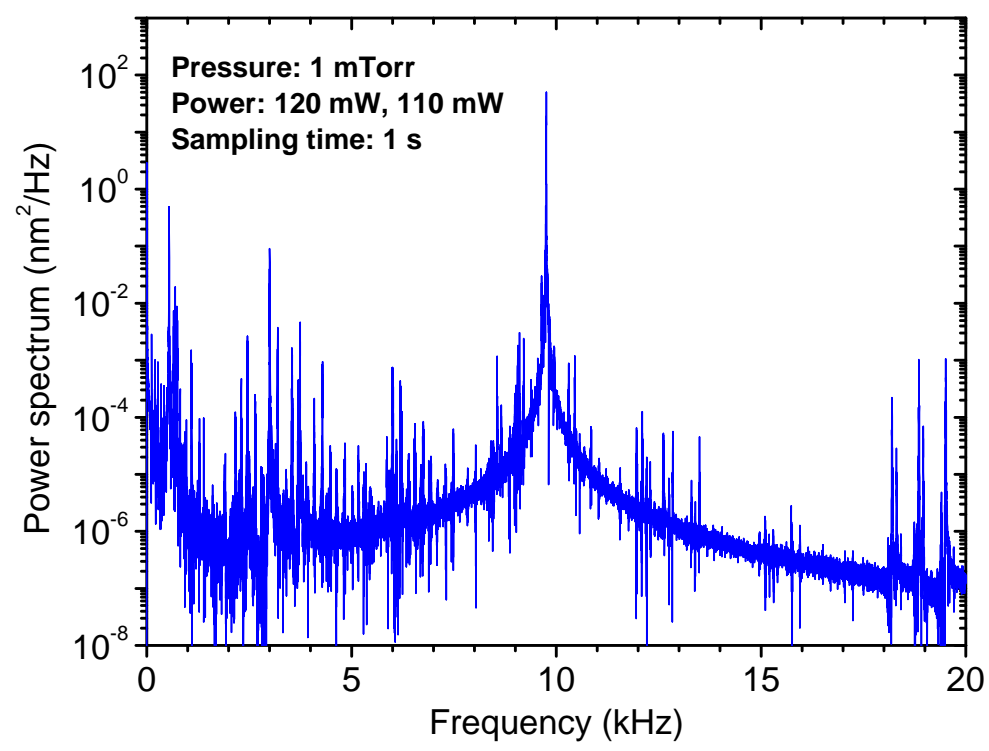

Figure 6.2: The power spectrum of a trapped 3.0- $\mu \mathrm{m}$ diameter microsphere at 1 mtorr. 
$\left(Q_{j}=\omega_{j} / \Gamma_{0}\right)$ of $2.1 \times 10^{4}$. This implies the power fluctuation of the trapping laser is smaller than $0.01 \%$ during the measurement. An optically trapped microsphere provides a method to directly convert laser power to a frequency signal, which can be measured precisely. Stabilization of laser power to a trapped bead can find applications in laser physics, and can enable a more precise measurement of the Q for a second trapped bead in vacuum.

Before feedback cooling, we observe a sharp transition in the trap lifetime as a function of pressure. Above the transition pressure, the microsphere can be trapped stably for many hours. Below the transition pressure, the microsphere is lost within a few seconds. This is because of the heating effect due to the light scattering, which has been discussed in the previous section. The transition pressure depends critically on the alignment of the two counterpropagating trapping beams. We can reduce it to less than $0.1 \mathrm{~Pa}$ by aligning the two laser beams. Thus $\alpha_{j}$ in Eq. 6.12 can be smaller than $2 \pi \times 0.5 \mathrm{~Hz}$. We also observed limit cycles (vortices) in the motion of a trapped microsphere when the trapping beams are intentionally misaligned. A 3D simulation of the system is required in order to fully understand these phenomena.

\subsection{A 3D split detection system}

In order to monitor the motion of a trapped microsphere with ultrahigh precision in all three dimensions, we built a 3D split detection system. As shown in Fig. 6.3, the X, Y, and Z detectors are fast balanced photo-detectors with bandwidth of $75 \mathrm{MHz}$. They have two matched photodiodes to cancel the 
common mode noise in the laser beams, allowing ultra-high precision measurements of the position of a trapped microsphere. When a trapped microsphere moves in the horizontal (vertical) direction, it deflects the trapping beam in the horizontal (vertical) direction. This changes the relative power between the two beams after the MX (MY) mirror, which is measured by the $\mathrm{X}(\mathrm{Y})$ detector. The motion of a trapped bead along the trap axis changes the divergence angle of the output beam, which changes the waists of incident beams on the $\mathrm{Z}$ detector. One photodiode of the $\mathrm{Z}$ detector is smaller than the waist of the incident beam. It measures only part of the power of the incident beam. Thus its output voltage depends on the waist of the incident beam, which is a function of the position of the microsphere in $\mathrm{Z}$ axis. The other photodiode of the $\mathrm{Z}$ detector is much larger than the waist of the incident beam. It measures the total power of the incident beam. Thus its output voltage does not depend on the waist of the incident beam, and can serve as a reference signal [111]. A photo of our 3D detection system is shown in Fig. 6.4.

For small displacements of a trapped microsphere near the trap center, the voltage output $\left(U_{i}\right)$ of each detector is proportional to the displacement $\left(x_{i}^{D}\right)$ of the microsphere along the detection direction, i.e. $U_{i}=\beta_{i} x_{i}^{D}$, where $i=1,2,3$ denotes $\mathrm{X}, \mathrm{Y}$, and $\mathrm{Z}$ detectors, and $\beta_{i}$ is the calibration factor of the detector. We align the detection system carefully to make the detection direction of each detector be parallel to one of the trap's fundamental mode axes. In reality, however, there is always slight difference between the detection directions and the fundamental mode axes. Thus the voltage output from each 
detector is a combination of signals from each mode, i.e. $U_{i}=\beta_{i}\left(\alpha_{i 1} x_{1}^{M}+\right.$ $\left.\alpha_{i 2} x_{2}^{M}+\alpha_{i 3} x_{3}^{M}\right)$, where $x_{j}^{M}(j=1,2,3)$ is the displacement of the microsphere along the fundamental mode axis $\hat{x}_{j}^{M}$, and $\alpha_{i j}$ is the projection coefficient of

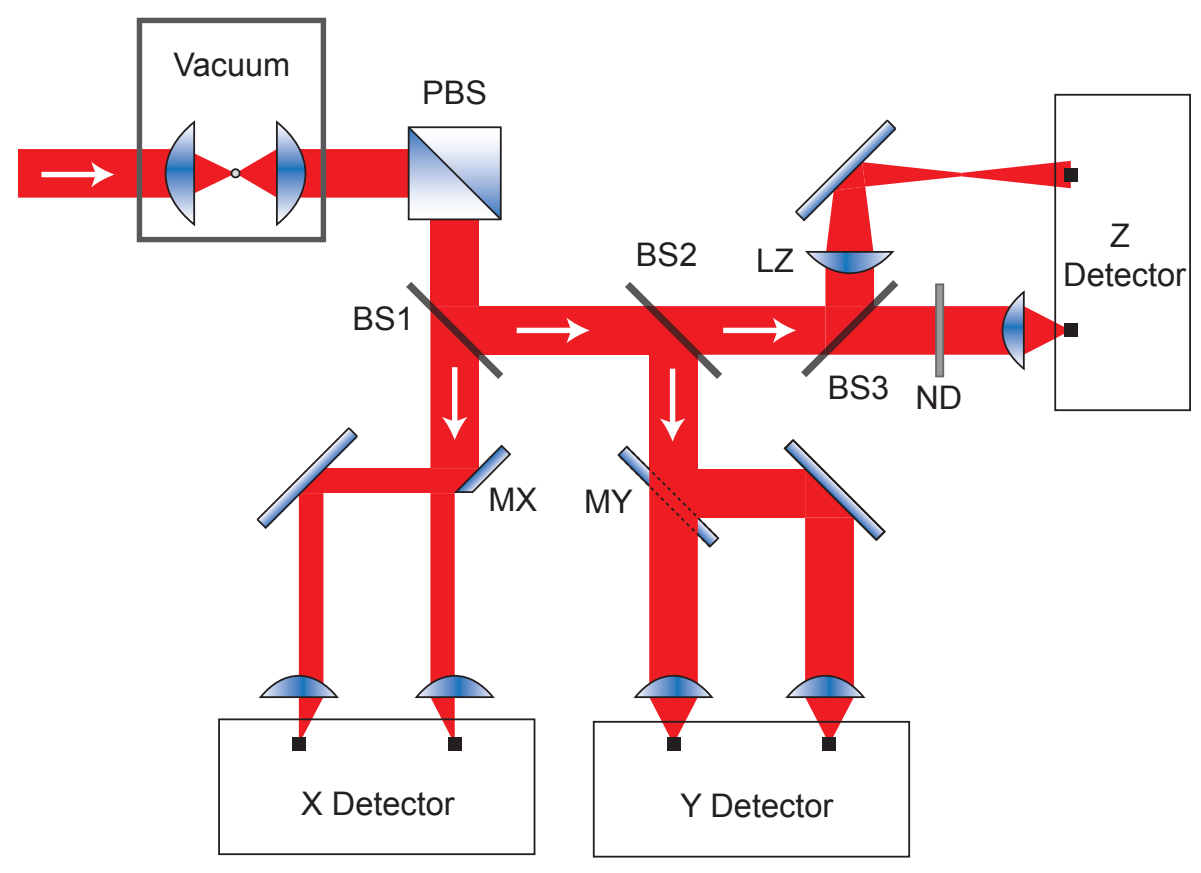

Figure 6.3: Simplified schematic showing the detection system that can monitor the real-time position of a trapped microsphere with ultra-high precision in all three dimensions. One of the trapping beams (the other trapping beam is not shown) passes through a trapped microsphere inside a vacuum chamber and is reflected by a polarizing beam splitter cube (PBS). It is then split to three beams by two beam splitters (BS1 and BS2) for 3D detection. MX is a mirror with a sharp edge that splits the beam into two parts horizontally. MY is a mirror with a sharp edge that splits the beam into two parts vertically. BS3 is a beam splitter, ND is a neutral density filter, and LZ is a lens. The X, $\mathrm{Y}$, and $\mathrm{Z}$ detectors are balanced detectors that have two matched photodiodes to cancel the common mode noise in the laser beams. 
$\hat{x}_{j}^{M}$ to the detection direction $\hat{x}_{i}^{D}$. Usually only one term dominates as the detection directions are almost parallel to the mode axes.

The expected value of the power spectrum of the voltage output from each detector is [76]:

$$
S_{i}^{U}(\Omega) \equiv\left\langle\left|\widetilde{U}_{i}\right|^{2} / T_{m s r}\right\rangle=\beta_{i}^{2}\left\langle\left|\alpha_{i 1} \widetilde{x}_{1}^{M}+\alpha_{i 2} \widetilde{x}_{2}^{M}+\alpha_{i 3} \widetilde{x}_{3}^{M}\right|^{2} / T_{m s r}\right\rangle,
$$

where $T_{m s r}$ is the measurement time, $\widetilde{U}_{i}$ and $\widetilde{x}_{j}^{M}$ are Fourier transforms of $U_{i}$ and $x_{j}^{M}$, respectively. The expansion of $S_{i}^{U}(\Omega)$ has 9 terms, but the expected values of the cross correlation terms $\left\langle\widetilde{x}_{i}^{M} \widetilde{x}_{j}^{M}\right\rangle(i \neq j)$ are 0 when there is no feedback, because the three components of the motion of a microsphere in

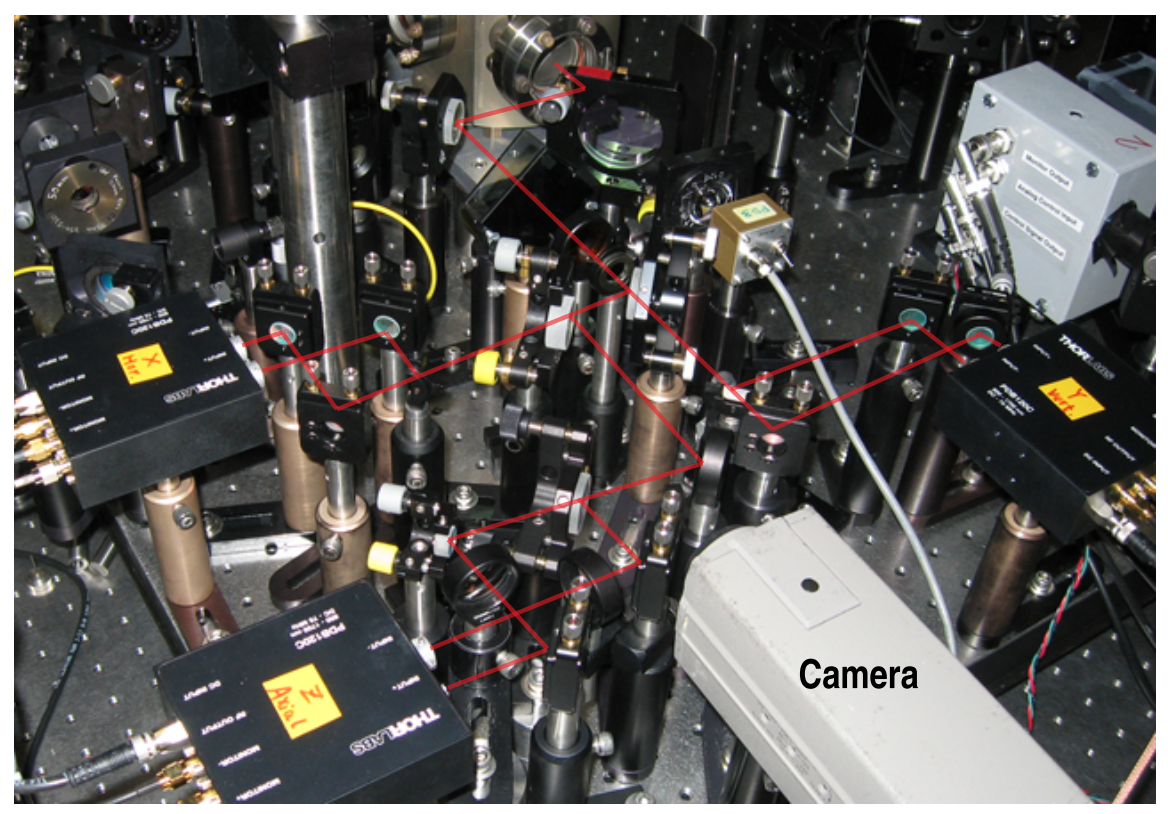

Figure 6.4: A photo of our 3D detection system and a camera for imaging a trapped microsphere with the scattered light. Red lines are drawn on the photo to show the light paths of the detection system. 
a harmonic trap are uncorrelated. With active feedback, there can be small correlations between different directions if the feedback loops are coupled. In real experiments, the coupling is small and the trapping frequencies are different along different directions, thus we assume that the average values of the cross correlations are negligible. Then

$$
S_{i}^{U}(\omega)=\beta_{i}^{2}\left[\alpha_{i 1}^{2} S_{1}(\omega)+\alpha_{i 2}^{2} S_{2}(\omega)+\alpha_{i 3}^{2} S_{3}(\omega)\right]
$$

where $S_{j}(\omega)$ is the power spectrum of COM motion along each fundamental mode axis. $S_{j}(\omega)$ is described by Eq. 6.2 without feedback cooling, and by Eq. 6.5 with feedback cooling.

The detection system can be calibrated by fitting the measured power spectra at room temperature with the expected power spectra $S_{i}^{U}(\omega)$ to obtain calibration factor $\beta_{i}^{2} \alpha_{i j}^{2}$ for each mode that is distinguishable in the power spectra. We can also obtain $\beta_{i}^{2}$ directly by the energy equipartition theorem, which says $\left\langle M v_{i}^{2} / 2\right\rangle=k_{B} T_{0} / 2$, where $v_{i}$ is the instantaneous velocity of the microsphere projected onto any axis. Since $U_{i}=\beta_{i} x_{i}^{D}$, we have $\beta_{i}^{2}=\frac{M}{k_{B} T_{0}}\left\langle\left(\frac{d U_{i}}{d t}\right)^{2}\right\rangle$. With $\beta_{i}^{2} \alpha_{i j}^{2}$ and $\beta_{i}^{2}$, we can easily obtain $\alpha_{i j}^{2}$ to check the alignment of our detection system. In the experiment, each detector is used to monitor only one mode, so only three calibration factors $\left(\beta_{1}^{2} \alpha_{11}^{2}, \beta_{2}^{2} \alpha_{22}^{2}\right.$, and $\left.\beta_{3}^{2} \alpha_{33}^{2}\right)$ are required for measuring the mode temperatures with feedback cooling.

The mass of the microsphere is required to obtain the calibration factors. The pure silica $\left(\mathrm{SiO}_{2}\right)$ microspheres used in this experiment are from Bangs Laboratories, Inc. Their mean diameter is $3.0 \mu \mathrm{m}$, corresponding to a 
mean mass of $2.8 \times 10^{-14} \mathrm{~kg}$ for each microsphere. The standard deviation of the size given by the supplier is $14 \%$. The exact diameter of the microsphere is not important for feedback cooling. The temperatures of the feedback-cooled motion are obtained by comparing the power spectra of the same microsphere with and without feedback cooling, a measurement which is independent of the exact size of the microsphere. The viscous damping factor $\left(\Gamma_{0}\right)$ of a microsphere in air, however, depends on the size of the microsphere. Using the measured damping factor shown in Fig. 6.1, we obtain the diameter of a microsphere by kinetic theory (Eq. 6.14) to be $2.7 \mu \mathrm{m}$, which is within the uncertainty range given by the supplier.

\subsection{D optical feedback cooling}

Figure 6.5 shows the first feedback cooling scheme that we used in our experiment. The position signal of a trapped microsphere is sent through a preamplifier that has a bandpass filter, and a derivative circuit $(\mathrm{d} / \mathrm{dt})$ to provide a signal proportional to the velocity of the microsphere. This velocity signal is used to control the frequency of the output signal of a radio frequency (RF) deflector driver (IntraAction, Model: DE-802M26). The output of the driver is a single frequency signal in the range of $60-100 \mathrm{MHz}$. The driver has an analog input to modulate the frequency of the signal. An analog input signal from $0 \mathrm{~V}$ to $1 \mathrm{~V}$ will change the frequency of the output signal by 40 MHz. The RF signal drives an acousto-optic modulator (AOM) which controls

the p-polarized trapping beam. The frequency of the RF signal determines the 
direction of the laser beam, and the power of the RF signal determines the power of the laser beam. Here we do feedback cooling by changing the direction of one of the trapping beams in the horizontal direction. The laser beam will exert a force on the microsphere as a function of its velocity in the horizontal direction, which cools the motion of the microsphere.

The preamplifier (SR560, Stanford Research Systems) has a very low input noise (about $4 \mathrm{nV} / \sqrt{\mathrm{Hz}}$ ), and a variable gain from 1 to $5 \times 10^{4}$. In this

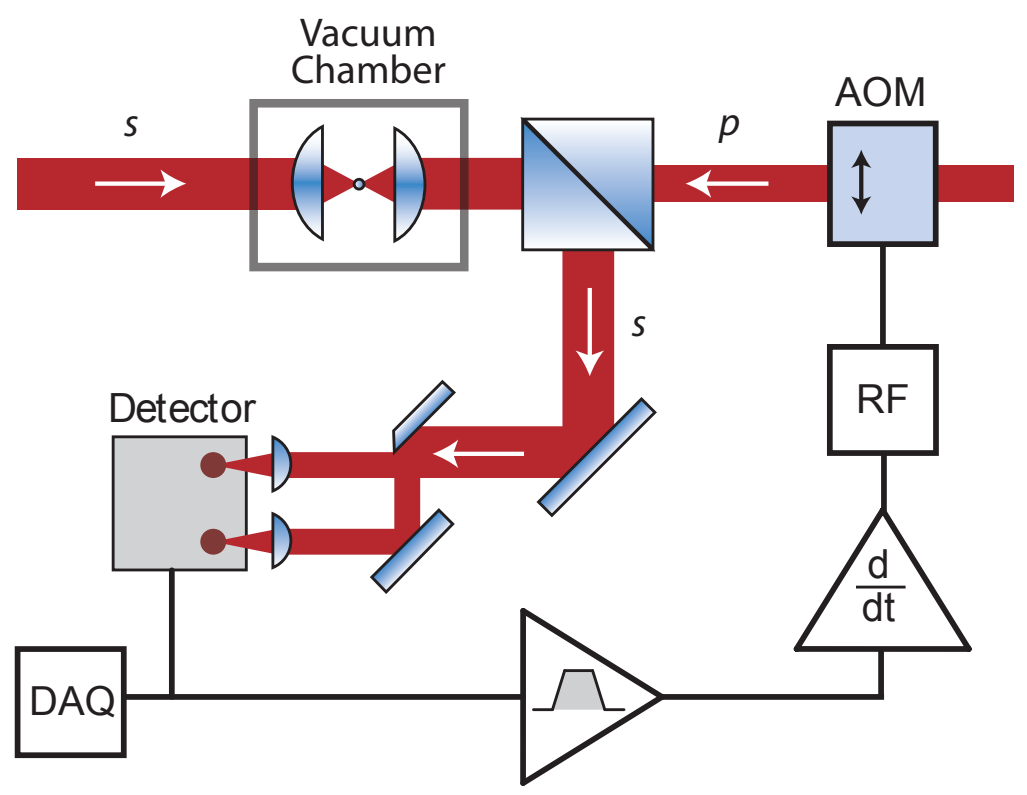

Figure 6.5: Diagram of a 1D feedback cooling scheme. The position of a trapped microsphere is monitored by a home-built detection system. The position signal is sent through a preamplifier which has a bandpass filter (typically $100 \mathrm{~Hz}$ to $300 \mathrm{kHz}$ ), and a derivative circuit (d/dt) to provide a signal proportional to velocity. This velocity signal is used to control the frequency of a radio frequency $(\mathrm{RF}) \mathrm{AOM}$ driver which modulates the direction of the laser beam. The data is digitized and stored on a computer by a data acquisition card (DAQ). 
experiment, we only need the gain to be about 2 or 5 because the output signal from the detector is pretty big already. The preamplifier contains two firstorder RC filters that can be set to -3 dB cutoff frequencies chosen from a 1-3-10 sequence, from $0.03 \mathrm{~Hz}$ to $1 \mathrm{MHz}$. We typically set the low cutoff frequency to be $100 \mathrm{~Hz}$ and the high cutoff frequency to be $300 \mathrm{kHz}$. The preamplifier also has a function to invert the signal, which is very useful. The derivative circuit is simply the 'derivative' branch of an analog PID controller. We first used a commercial PID controller (SIM 960, Stanford Research Systems). It has a lot of powerful functions, including digital control of the P, I, D, and Offset. However, it picks up some external electronic noise through its power cord. We eventually decided to use our home-built derivative circuit for feedback cooling.

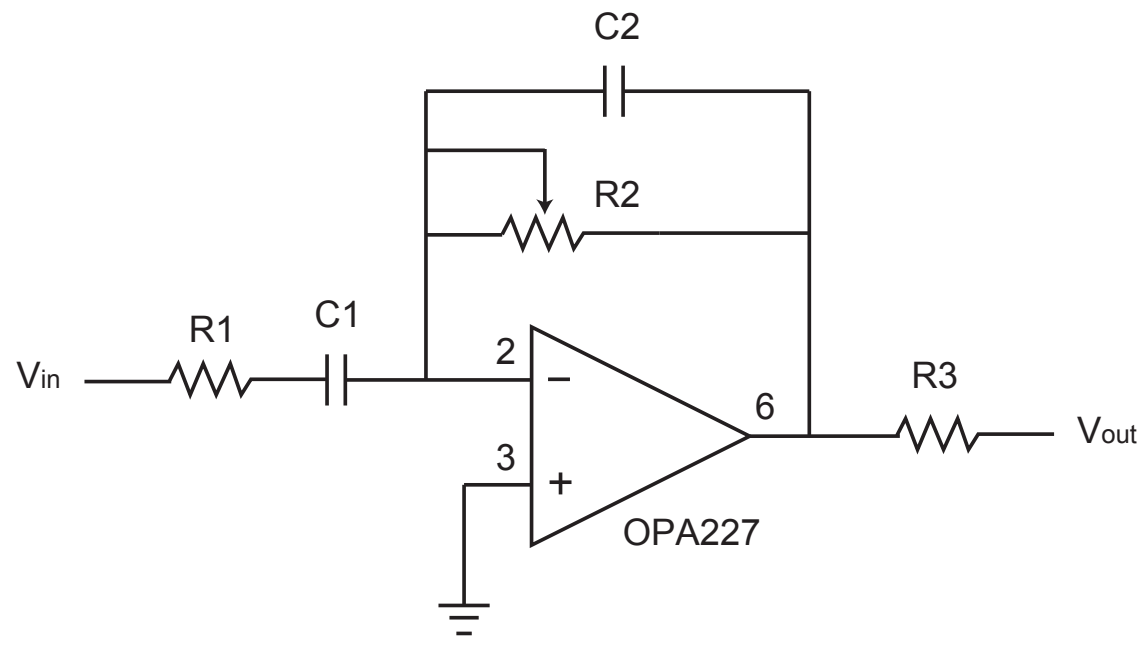

Figure 6.6: A derivative circuit for calculating the real-time velocity of a microshpere from its position signal. 
A detailed description of our home-built PID controllers can be found in Appendix C.1 of the Ph.D dissertation of Todd Meyrath [112]. The 'derivative' part of the circuit is shown in Fig. 6.6. The main component of the circuit is the low-noise operational amplifier, the capacitor $\mathrm{C} 1$ and the tunable resistor R2. The operational amplifier is OPA227 from Texas Instruments. It has a low noise level of $3 \mathrm{nV} / \sqrt{\mathrm{Hz}}$ and a wide bandwidth of $8 \mathrm{MHz}$. The differentiation time of the derivative circuit is $\mathrm{C} 1 \times \mathrm{R} 2$. It is important that the OPA227 is unity-gain stable. We have tried to use an OPA228 amplifier which is faster than the OPA227, but the circuit is not as stable for low gain. The resistor $\mathrm{R} 1$ is much smaller than R2, and the capacitor $\mathrm{C} 2$ is much smaller than $\mathrm{C} 1$. $\mathrm{R} 1$ limits the differential gain, and $\mathrm{C} 2$ gives high frequency roll-off. R1 and $\mathrm{C} 2$ are necessary because the derivative circuit has a very large gain at high frequencies. For a pure derivative circuit, if the input signal is $V_{i n}=\sin (\omega t)$, then the output will be $V_{\text {out }}=\omega \cos (\omega t)$, which will be infinite if $\omega$ is infinite. Thus a derivative circuit amplifies high frequency noise. So we use R1 to limit the gain, and $\mathrm{C} 2$ to serve as a low-pass filter to reduce the gain of high frequency noise. $\mathrm{R} 3$ is used to isolate the derivative circuit from other parts of the circuit so that they do not interfere with each other. Typical values of the resistors and capacitors are $\mathrm{R} 1=50 \Omega, \mathrm{C} 1=2.2 \mathrm{nF}, \mathrm{R} 2=0-50 \mathrm{k} \Omega, \mathrm{C} 2=0.1 \mathrm{nF}$, and $\mathrm{R} 3=1 \mathrm{k} \Omega$.

Figure 6.7 shows some results of the 1D feedback cooling. In Fig. $6.7(\mathrm{~A})$, the rms velocity of the microsphere is reduced from $0.43 \mathrm{~mm} / \mathrm{s}$ to $0.090 \mathrm{~mm} / \mathrm{s}$ by 1D feedback cooling. The temperature of the Brownian motion 
(A)

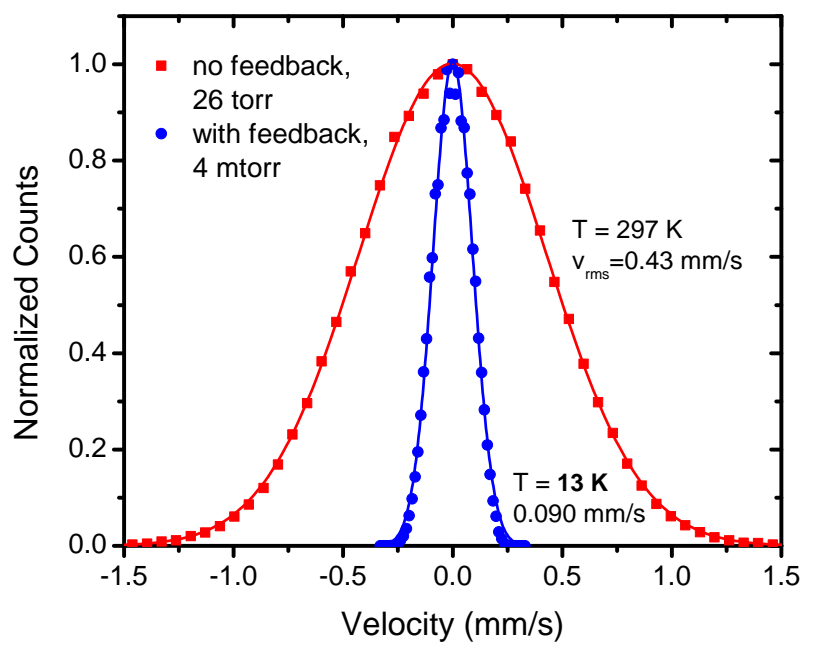

(B)

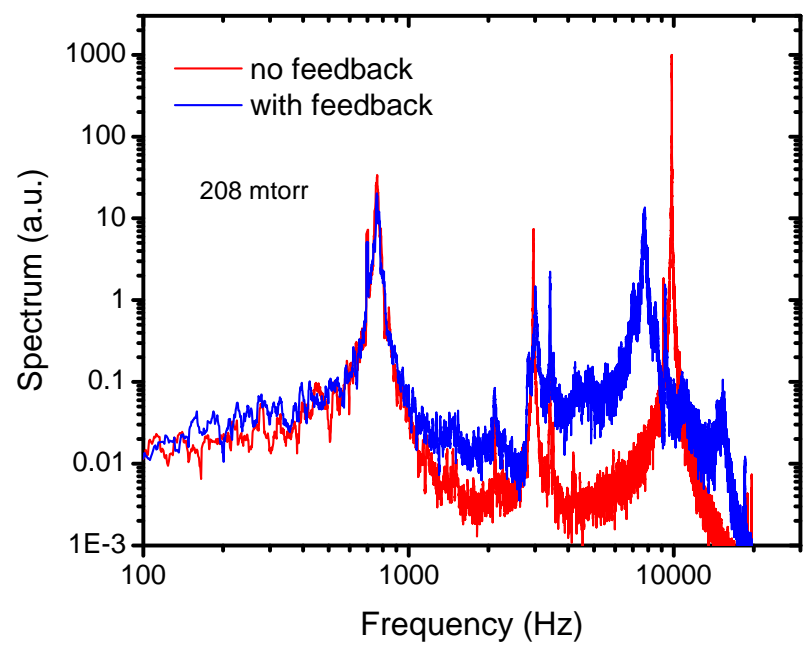

Figure 6.7: (A): The normalized velocity distributions of a trapped 3.0- $\mu \mathrm{m}$ diameter microsphere without feedback cooling at 26 torr (red curve), and with feedback cooling at 4 mtorr (blue curve). (B): The power spectra of the microsphere with (blue curve) and without (red curve) feedback cooling at 208 mtorr. 
is reduced from room temperature $(297 \mathrm{~K})$ to $13 \mathrm{~K}$. Fig. 6.7(B) shows the power spectra of the microsphere with (blue curve) and without (red curve) feedback cooling at 208 mtorr. With 1D feedback cooling, the peak near 10 $\mathrm{kHz}$ is reduced by 2 orders, while the peaks near $800 \mathrm{~Hz}$ and $3 \mathrm{kHz}$ do not change very much. Thus the $1 \mathrm{D}$ feedback cooling only cools the motion along one direction efficiently.

In the red curve of Fig. 6.7(B), the peak at $10 \mathrm{kHz}$ corresponds to the mode along the $\mathrm{X}$ axis, the tiny peak at $9 \mathrm{kHz}$ corresponds to the mode along the $\mathrm{Y}$ axis. These two modes have almost the same frequency because the laser beam is only slightly elliptical. The peak at $3 \mathrm{kHz}$ corresponds to the $\mathrm{Z}$ mode. We initially thought the peak at $800 \mathrm{~Hz}$ corresponds to the $\mathrm{Z}$ mode. After an extensive study, we believe that the $800 \mathrm{~Hz}$ peak is because of
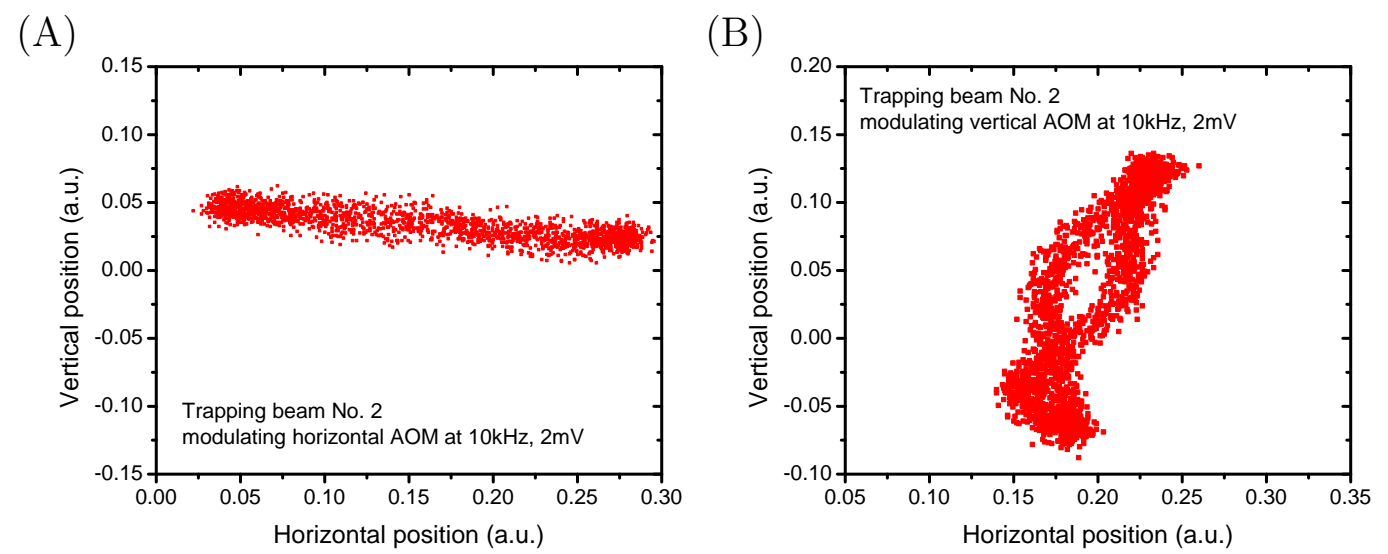

Figure 6.8: (A): Modulation of the direction of beam No. 2 (the p-polarized beam) with an AOM in the horizontal direction. (B): Modulation of the direction of beam No. 2 with an AOM in the vertical direction. 
the frequency difference between the $\mathrm{X}$ and $\mathrm{Y}$ mode. This became clear when we do $3 \mathrm{D}$ feedback cooling. The $\mathrm{Z}$ cooling beam reduced the $3 \mathrm{kHz}$ mode efficiently, but did not reduce the $800 \mathrm{~Hz}$ peak efficiently. The frequency (800 $\mathrm{Hz}$ ) equals the frequency difference between the $\mathrm{X}$ and $\mathrm{Y}$ mode. We are also able to make the $800 \mathrm{~Hz}$ peak disappear by better alignment.

We later installed another AOM to control the direction of beam No. 2 (the $p$-polarized beam) in the vertical direction. Thus we can do 3D feedback cooling by modulating the intensity of beam No. 2, and the directions of beam No. 2 in horizontal and vertical directions. The beam No. 1 (the $s$-polarized beam) is not modulated because it is used for detection. We have tried such 3D feedback cooling method, but we were only able to cool the motion from $297 \mathrm{~K}$ to about $10 \mathrm{~K}$.

We finally found out the problem after struggling for several months. In order to find out the problem, we use a quadrant detector to monitor the motion of a laser beam when we modulate its direction by an AOM. Figure 6.8 shows the motion of the center of beam No. 2 when we modulate its directions with an AOM (IntraAction, model: ATM-801A2) in the horizontal direction (A) and an AOM in the vertical direction (B). We use a sine signal with peakto-peak voltage of $2 \mathrm{mV}$ at $10 \mathrm{kHz}$ to drive the analog input of the $\mathrm{RF}$ driver. This modulates the frequency of the RF signal (centered at about $80 \mathrm{MHz}$ ) by $80 \mathrm{kHz}$. Thus the frequency of the RF signal is $80 \mathrm{MHz}+40 \mathrm{kHz} \cdot \sin \left(2 \pi 10^{4} t\right)$, where $t$ is time. Ideally, the data points should be a straight horizontal line in Fig. 6.8(A) and a straight vertical line in Fig. 6.8(B). However, the experi- 
mental points are not in a straight line, especially in Fig. 6.8(B). This means that when we use an AOM to control the laser along the vertical direction, the laser beam also moves along the horizontal direction in a very complex way. Thus when we cool the motion vertically, we also heat the motion horizontally. This limits the final temperature of the feedback cooling with this method.

Figure 6.9 shows the motion of the center of beam No. 1 (the $s$-polarized beam) when we modulate its direction with an AOM (Isomet, model: 1205C2-804) in the horizontal direction. We want it to be a straight line in the horizontal direction. However, the real motion of the laser beam is very complex.

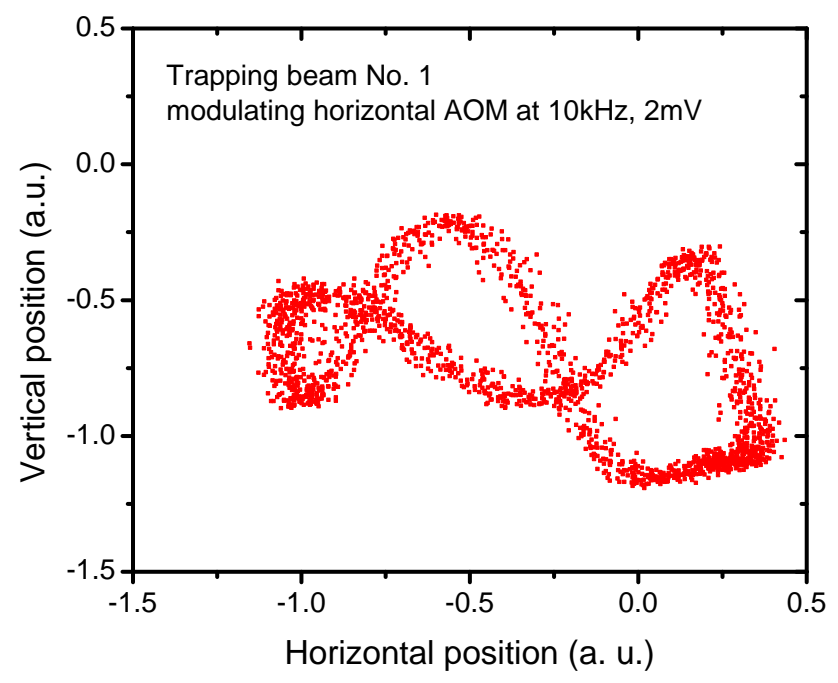

Figure 6.9: Modulation of the direction of beam No. 1 with an AOM in the horizontal direction. 


\subsection{Electrostatic forces}

After we found the problem of modulating the direction of a laser beam with an AOM, we decided to try to perform feedback cooling using electrostatic forces. The natural charge of a microsphere is negligible. It is necessary to charge the microsphere first in order to apply sufficient electrostatic forces for feedback cooling.

Fig. 6.10 shows our setup for air discharge and feedback cooling with an electrostatic force. The stainless steel mounts of the two aspheric lenses and the whole vacuum chamber are grounded. A thin stainless steel sheet is inserted half way between the two lenses. It is connected to a high-voltage amplifier that can deliver a $0-1 \mathrm{kV}$ voltage output. There will be a strong

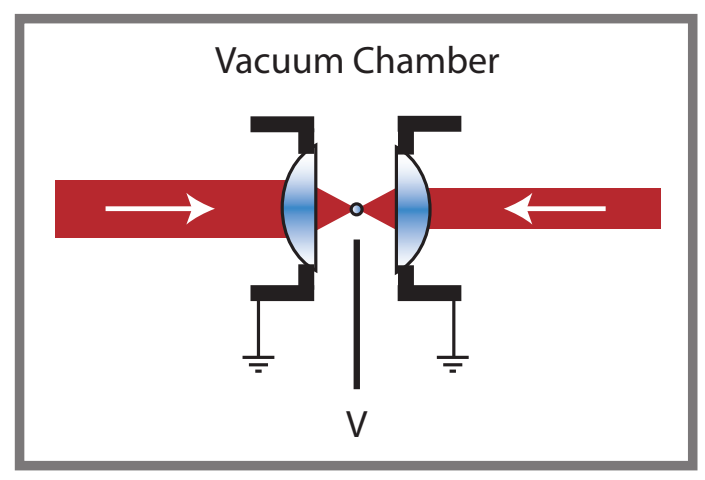

Figure 6.10: Setup for air discharge and feedback cooling with an electrostatic force. The stainless steel holders of the two aspheric lenses and the whole vacuum chamber are grounded. The smallest separation between the two holders is about $4 \mathrm{~mm}$. A thin stainless steel sheet is inserted at the center of the two lenses as an electrode. The edge of the steel sheet is about $1 \mathrm{~mm}$ away from where the microsphere is trapped. The steel sheet is connected to a high-voltage amplifier that can deliver $0-1 \mathrm{kV}$ voltage output. 


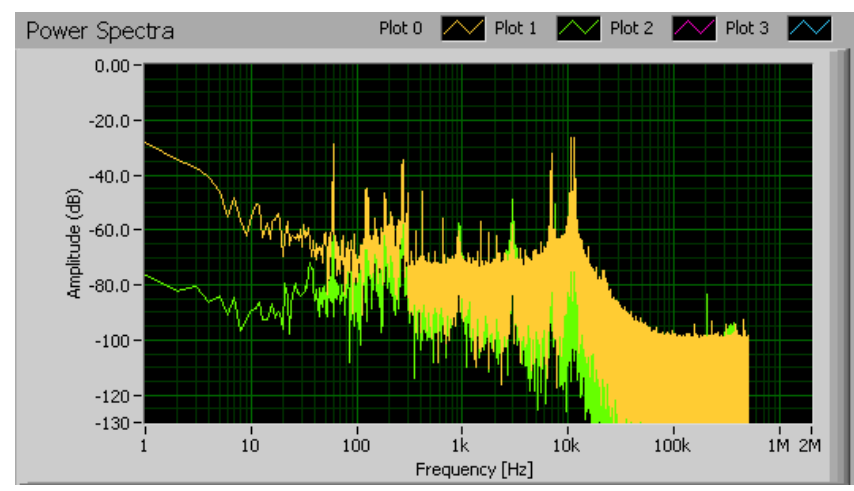

(A)

before discharge

$1.5 \mathrm{kHz}, 340 \mathrm{~V}$

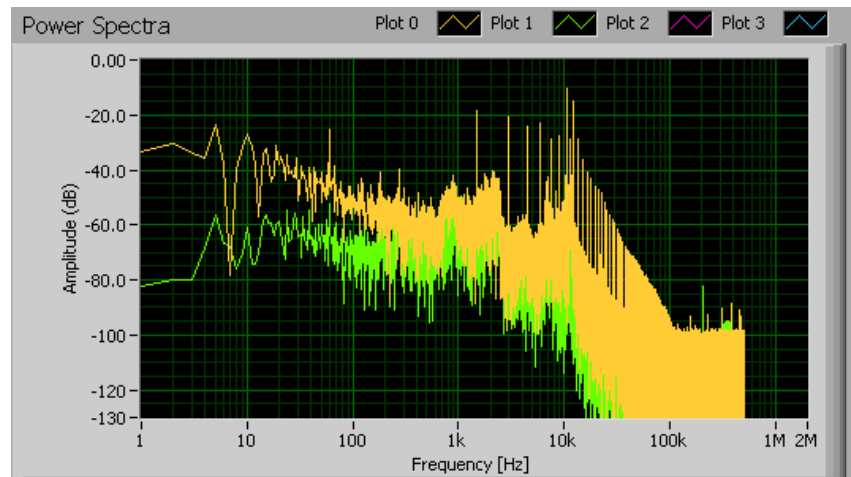

(B)

during discharge

$1.5 \mathrm{kHz}, 680 \mathrm{~V}$

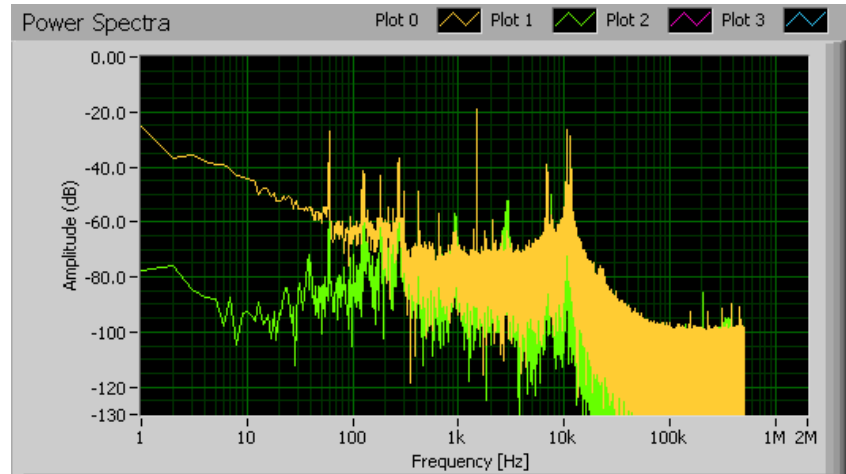

(C)

after discharge $1.5 \mathrm{kHz}, 340 \mathrm{~V}$

Figure 6.11: Spectra of the motion of a trapped microsphere before (A), during (B) and after (C) air discharge. The pressure is 461 mtorr. The frequency of the AC voltage is $1.5 \mathrm{kHz}$, and the air discharge happens when the peak voltage is about $680 \mathrm{~V}$. We reduce the peak voltage in several seconds after air discharge occurs to avoid the loss of the microsphere. 
electric field between the steel sheet and the steel holders when a high voltage is applied to the steel sheet. At high voltage, the air breaks down and becomes conductive. This can damage the high-voltage amplifier. We connect a $1 \mathrm{M} \Omega$ resister in series with the steel sheet and the high-voltage amplifier to limit the peak current.

We first trapped a microsphere with the dual-beam optical trap at about 100 torr. We then reduced the pressure to about 0.5 torr. With the microsphere trapped, we applied an AC voltage in the form of $V(t)=$ $V_{0}[\sin (2 \pi f t)+1] / 2$ to the steel sheet. The frequency of the AC voltage was typically about $1 \mathrm{kHz}$. We increased the peak voltage slowly from a few volts to several hundred volts until air discharge occured. A spectrum of the motion of a trapped microsphere at 461 mtorr driven by a $1.5 \mathrm{kHz}, V_{0}=340 \mathrm{~V}$ signal

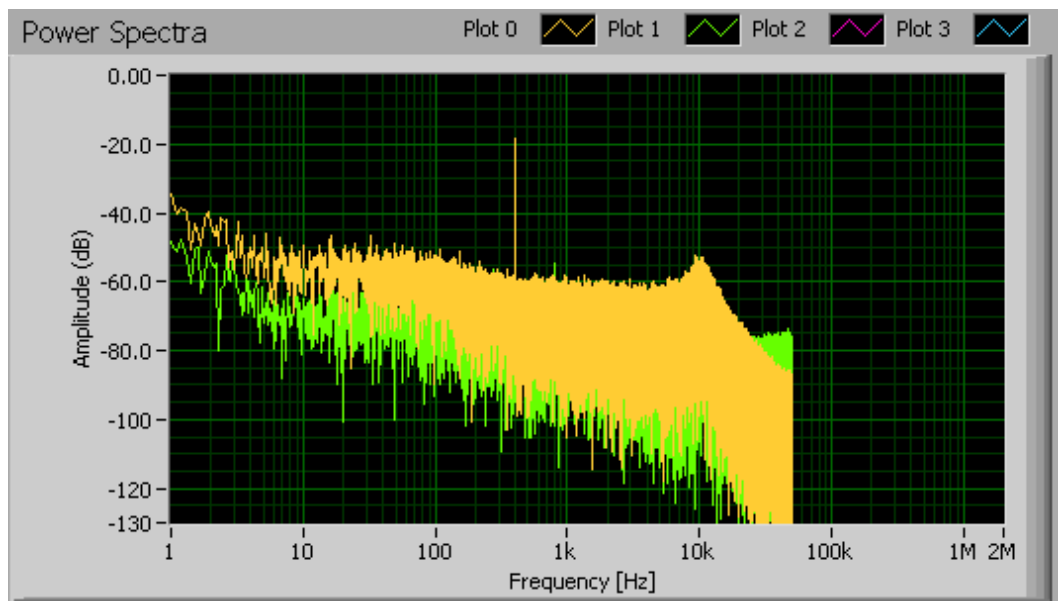

Figure 6.12: Spectrum of the motion of a trapped microsphere at 205 torr after air discharge. The micropshere is driven by a $400 \mathrm{~Hz}, V_{0}=510 \mathrm{~V} \mathrm{AC}$ voltage. After air discharge, the microsphere maintains its charge even at high pressure. 
is shown in Fig. $6.11 \mathrm{~A}$. The peak at $1.5 \mathrm{kHz}$ due to the $\mathrm{AC}$ signal is very small, because the natural charge of the microsphere is very small. When the peak voltage was increased to $680 \mathrm{~V}$, air discharge occurred. The microsphere moved violently during the air discharge. Thus there are a lot of peaks in Fig. 6.11B. It is surprising to us that the optical tweezer is stable enough to trap a microsphere during air discharge. We reduced the peak voltage in a few seconds after air discharge occured to avoid the loss of the microsphere. The microsphere gained charge during air discharge. Fig. 6.11C shows a spectrum of the motion of the microsphere driven by a $1.5 \mathrm{kHz}, V_{0}=340 \mathrm{~V}$ signal after air discharge. The peak at $1.5 \mathrm{kHz}$ after discharge is about 4 orders higher than the peak before discharge.

After air discharge, the microsphere maintains its charge, even at high pressures. Fig. 6.12 shows a spectrum of the motion of a trapped microsphere at 205 torr after air discharge. The microsphere is driven by a $400 \mathrm{~Hz}, V_{0}=$ $510 \mathrm{~V}$ AC voltage. The hight of the peak at $400 \mathrm{~Hz}$ is proportional to the square of the charge of the microsphere. By measuring the height of the peak, we can monitor the charge of the microsphere as a function of time. Fig. 6.13 displays the charge of the microsphere as a function of time over a period of 2 hours. The charge fluctuates because there is a weak air discharge near the microsphere. So the microsphere gains and losses charges over time. The shape of the curve depends on the driving voltage and the air pressure. We have not been able to observe individual steps in the curve when the microsphere gains or loses one electron. 
According to Coulomb's law, the force between two point charges $q_{1}$ and $q_{2}$ is:

$$
F=k_{e} \frac{q_{1} q_{2}}{r^{2}}
$$

where $k_{e}=8.99 \times 10^{9} \mathrm{~N} \cdot \mathrm{m}^{2} \mathrm{C}^{-2}$ is the Coulomb constant, and $r$ is the distance between the two point charges. The force between two electrons separated by $1 \mathrm{~mm}$ and $1 \mu \mathrm{m}$ is $2.3 \times 10^{-22} \mathrm{~N}$ and $2.3 \times 10^{-16} \mathrm{~N}$, respectively.

When $N$ electrons $e$ are distributed homogeneously on the surface of a microsphere with radius $R$, the energy required to add another electron is about:

$$
E=k_{e} \frac{N e^{2}}{R}
$$

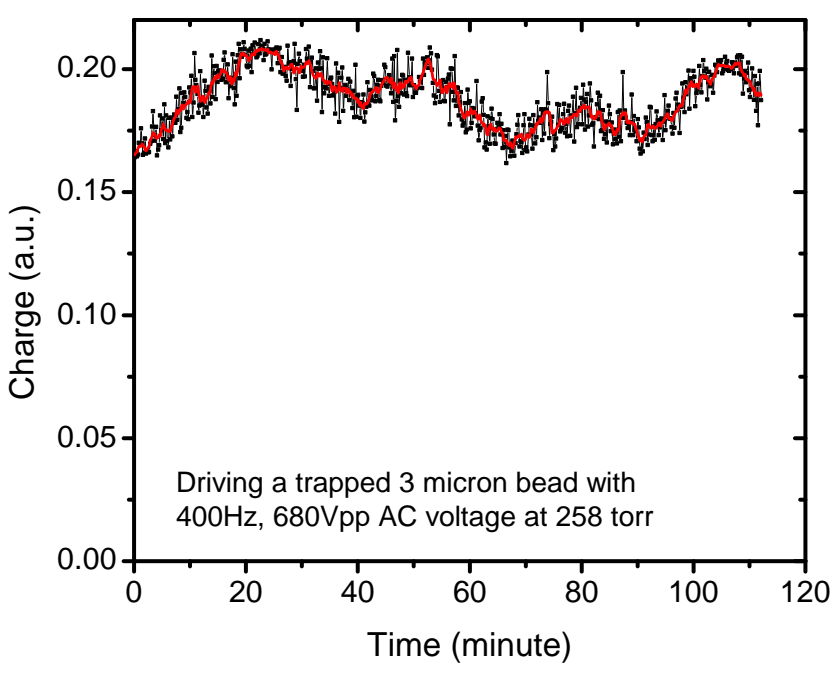

Figure 6.13: Fluctuation of the charge of a 3- $\mu$ m-diameter microsphere trapped at 205 torr, driven by a $400 \mathrm{~Hz}, V_{0}=680 \mathrm{~V}$ signal. a.u. : arbitrary unit. 
For a $R=1.5 \mu \mathrm{m}$ microsphere, the required energy is $k_{B} T / 2$ at room temperature when $\mathrm{N}=13$. Thus the natural charge of a $3-\mu \mathrm{m}$ diameter microsphere is in the order of $13 e$. We do not know the exact charge of the microsphere after air discharge, but estimate it to be on the order of $1000 e$ from the power spectrum (Fig. 6.11), which corresponds to $E=1 \mathrm{eV}$. The maximum electric field at the trap center is about $10 \mathrm{~V} / \mathrm{mm}$. Thus the maximum electrostatic force on the microsphere is in the order of $1.6 \mathrm{pN}$. This is consistent with the observed shift of the trap center when we apply a DC voltage.

The air discharge is not very controllable. This makes the current setup (Fig. 6.10) not suitable for feedback cooling. We have tried to implement feedback cooling with electrostatic forces and were able to cool the motion from room temperature to about $10 \mathrm{~K}$. The final temperature is limited by the fact that the charge of the microsphere fluctuates, and we do not know the real direction of the electrostatic force. These problems can be solved by a better design of the electrodes and a better charging method. The electrodes of a quadrupole ion trap [113] should be ideal for 3D feedback cooling with electrostatic forces. The microsphere can be charged by photoelectric charging with a ultraviolet lamp $[113,114]$, by using electrospray [115] or an electron gun. Combining an optical trap with an ion trap in the same location should be helpful in trapping and studying particles at ultrahigh vacuum. 


\subsection{Millikelvin cooling with 3D optical feedback}

Since significant efforts were required to improve feedback cooling with electrostatic forces, we decided to try feedback cooling with optical forces again. This time, we used AOM's to modulate the intensities of laser beams rather than the directions of laser beams to do 3D feedback cooling. This method turned out to work very well. It enables us to cool the center-ofmass motion of a trapped microsphere from room temperature to millikelvin temperatures in all three dimensions, with a minimum mode temperature of $1.5 \mathrm{mK}[15]$.

\subsubsection{Experimental setup}

A simplified scheme of our optical trap and cooling beams is shown in Fig. 6.14. The dual-beam optical trap is the same as described before. It is created inside a vacuum chamber by two counter-propagating laser beams focused to the same point by two identical aspheric lenses with a focal length of $3.1 \mathrm{~mm}$ and numerical aperture of 0.68 . The wavelength of both trapping beams is $1064 \mathrm{~nm}$. They are orthogonally polarized, and are shifted in

frequency to avoid interference. The beams are slightly elliptical and approximately form a harmonic trap with three fundamental vibration modes along the horizontal, vertical and axial directions, denoted X, Y, and Z in Fig. 6.14. The motion of a trapped bead causes deflection of both trapping beams. We monitor the position of the bead by measuring the deflection of one of the trapping beams with ultrahigh spatial and temporal resolution in all three 
dimensions (Fig. 6.3).

Using the position signal, we can calculate the instantaneous velocity of the bead, and implement feedback cooling by applying a force with a direction opposing the velocity (Fig. 6.15). The feedback is generated by scattering forces from three orthogonal $532 \mathrm{~nm}$ laser beams along the axes as shown in Fig. 6.3. The average intensity of the cooling beams is about $1 \%$ that of the trapping beams. The optical power of each cooling beam is controlled by an acousto-optic modulator (AOM). Each beam is modulated with a time-

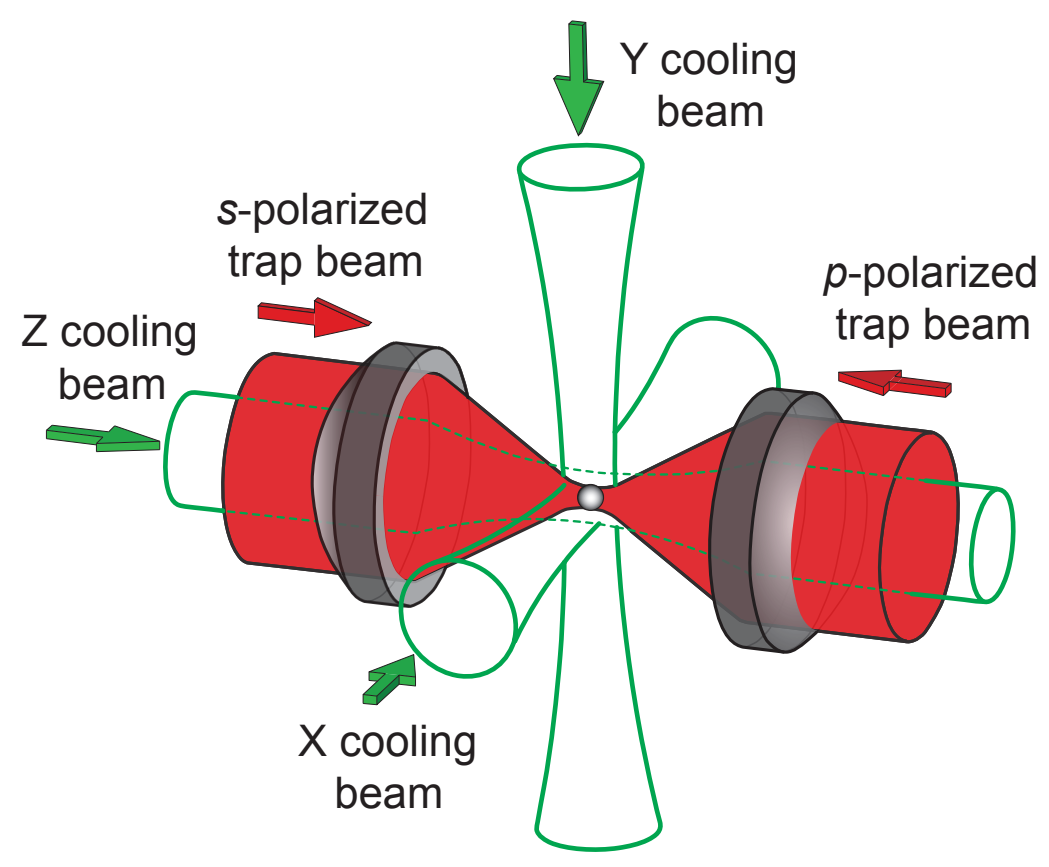

Figure 6.14: Simplified schematic showing a glass microsphere trapped at the focus of a counter-propagating dual-beam optical tweezer, and three laser beams along the axes for cooling. The wavelengths of the trapping beams and the cooling beams are $1064 \mathrm{~nm}$ and $532 \mathrm{~nm}$, respectively. 
varying signal proportional to the instantaneous velocity of the bead, added to an offset. The proportional component generates the required cooling force, while the offset slightly shifts the trap center. A photo of the optics of our 3D optical feedback cooling system is displayed in Fig. 6.16. The green color in the photo is due to the scattered light from the cooling beams. The trapping beams are infrared and cannot be seen in this photo.

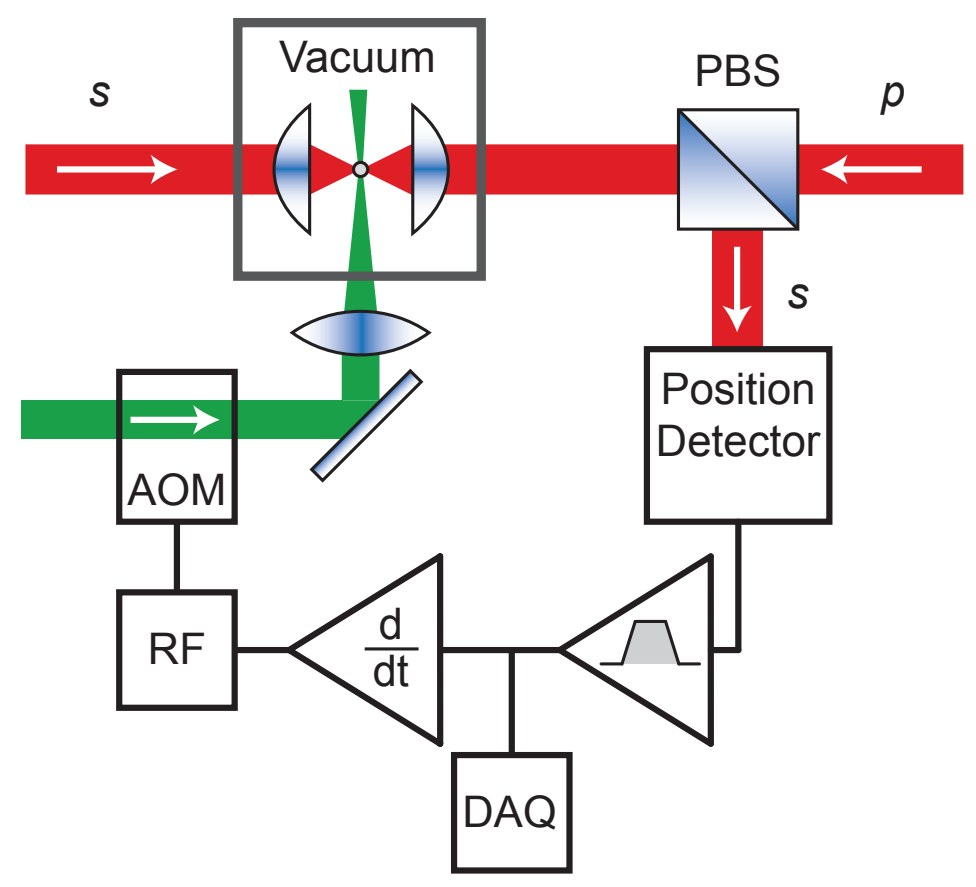

Figure 6.15: Diagram of the feedback mechanism for the $\mathrm{X}$ axis: The position of a trapped microsphere is monitored by a home-built detecting system. The position signal is sent through a bandpass filter (typically $100 \mathrm{~Hz}$ to $300 \mathrm{kHz}$ ) and a derivative circuit (d/dt) to provide a signal proportional to velocity. This velocity signal is used to control the output power of a radio frequency (RF) AOM driver which modulates the power of the $\mathrm{X}$ cooling beam. The data is digitized and stored on a computer by a data acquisition card (DAQ). 
Fig. 6.17 shows the power of the first order of a laser beam exiting the $\mathrm{AOM}$ as a function of the input voltage of the RF driver (IntraAction, model: ME-802) and the reading of the manual offset knob of the RF AOM driver. In general, the laser power is a nonlinear function of the input voltage. When the knob reading is at 5 , the laser power depends on the input voltage linearly around 0 . This is good for feedback cooling because we want the laser power to be proportional to the velocity of the microsphere. Thus we set the knob reading at 5 , and use the velocity signal as the input voltage to control the laser power for feedback cooling.

The behavior of the system with three dimensional (3D) feedback cool-

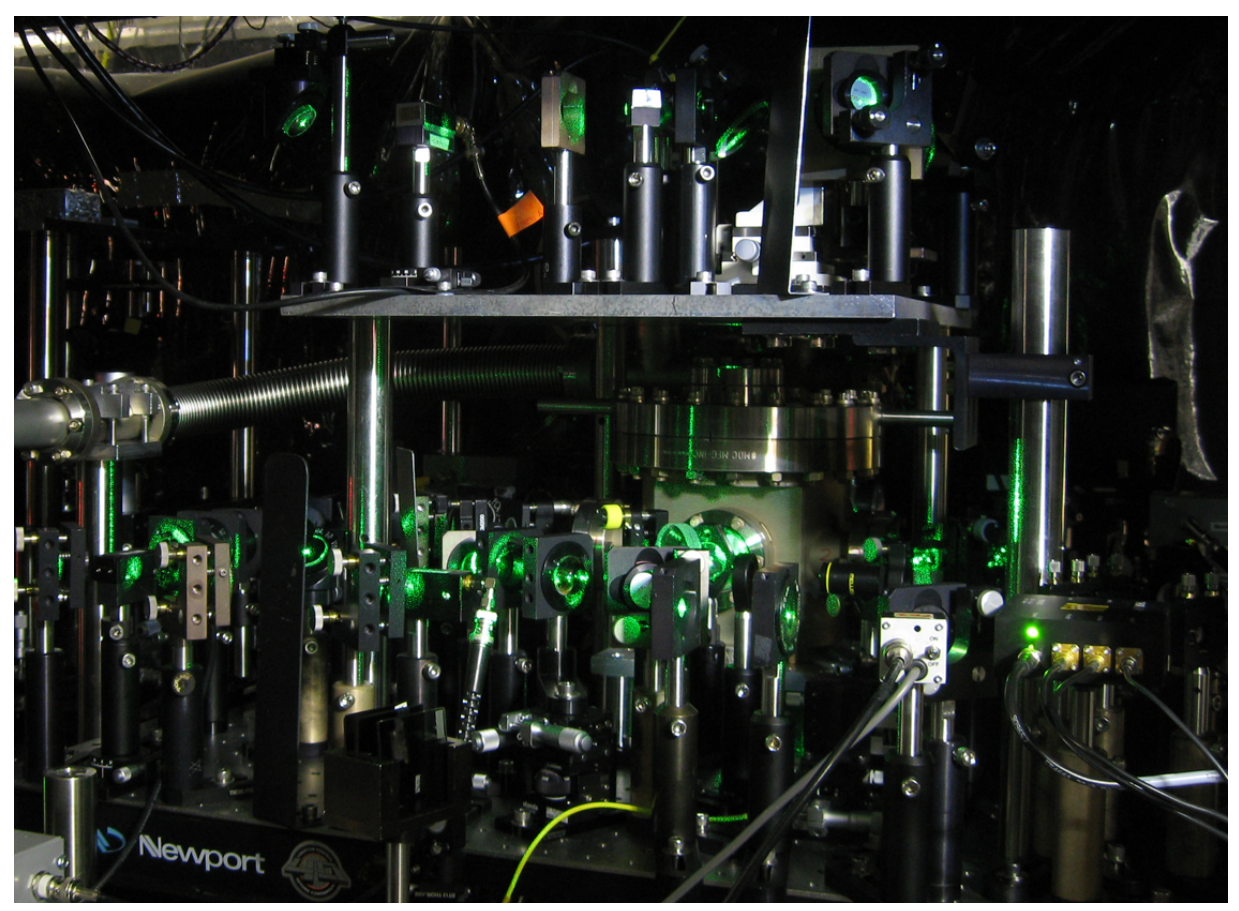

Figure 6.16: A photo of the 3D optical feedback cooling system. 
ing is straightforward to understand if we assume that there is no coupling between feedback forces and velocities in different directions. In this case, the feedback force in each direction adds an effective cold damping factor $\Gamma_{j}^{f b}$, and the total damping becomes $\Gamma_{j}^{t o t}=\Gamma_{0}+\Gamma_{j}^{f b}$. The power spectrum of the motion of a trapped microsphere with feedback cooling can be described by Eq. 6.5. The temperature of the motion with feedback cooling will be $T_{j}^{f b}=T_{0} \Gamma_{0} / \Gamma_{j}^{t o t}$. Thus the motion can be cooled significantly by applying feedback damping

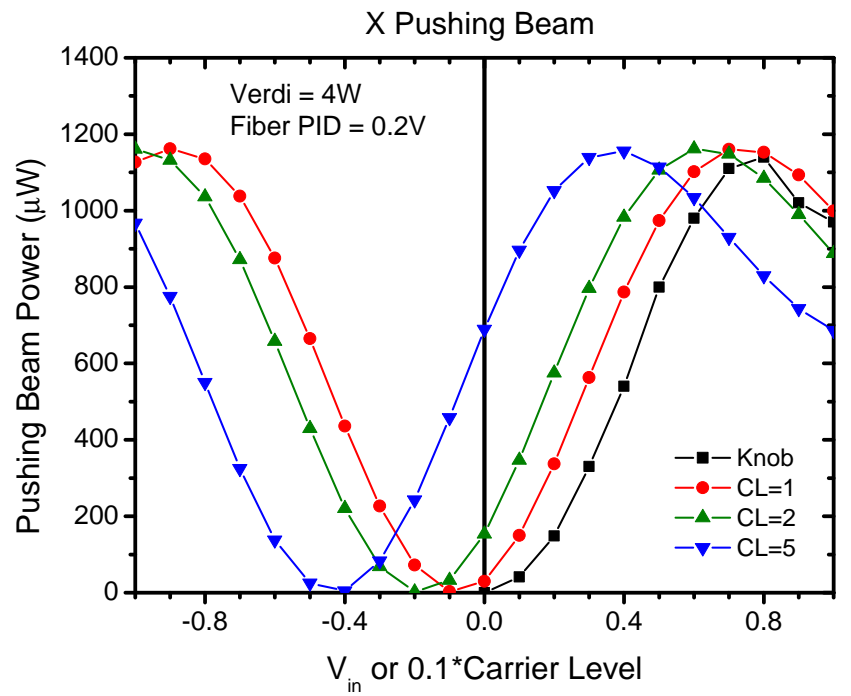

Figure 6.17: Control of the laser power with an AOM. The AOM is driven by a RF driver which uses an analog input to control the RF power electronically and a knob to tune the RF power manually. The analog input accepts a voltage from 0-1 V, and the knob has a reading (carrier level) from 0-10. For the black curve, the analog voltage input is zero, and the manual knob is tuned from 0 to 10. The other curves are the power of the laser as a function of the analog input when the knob is set at different readings (Red: knob reading at 1; Green: knob reading at 2; Blue: knob reading at 5 ). 
$\Gamma_{j}^{f b}>>\Gamma_{0}$. The lowest temperature will be limited by the noise in the detection system and feedback circuits, as well as coupling between different directions.

\subsubsection{Results of 3D optical feedback cooling}

Figures 6.18, 6.19 and 6.20 show experimental results of feedback cooling. Before feedback is turned on, the resonant frequencies $\left(\omega_{j} / 2 \pi\right)$ are $8066 \pm 5$ $\mathrm{Hz}, 9095 \pm 4 \mathrm{~Hz}$, and $2072 \pm 6 \mathrm{~Hz}$ for the fundamental modes at $637 \mathrm{~Pa}$ along the $\mathrm{X}, \mathrm{Y}$, and $\mathrm{Z}$ axes, respectively. At this pressure, the peaks in the power spectrum due to the three fundamental modes are distinguishable, and heating effects due to the laser are negligible. We can therefore use the measured power spectra at $637 \mathrm{~Pa}$ to calibrate the position detectors for the fundamental modes at room temperature. After we turn on feedback cooling, the temperature of the Y mode changes from $297 \mathrm{~K}$ to $24 \mathrm{~K}$ at $637 \mathrm{~Pa}$. The mode temperature is obtained by fitting the measured power spectrum with Eq. 6.6.

After switching on the feedback circuits, we reduce the air pressure while keeping the feedback gain almost constant, thus the heating rate due to collisions from air molecules decreases, while the cooling rate remains constant. As a result, the temperature of the motion drops. At $5.2 \mathrm{mPa}$, the mode temperatures are $150 \pm 8 \mathrm{mK}, 1.5 \pm 0.2 \mathrm{mK}$, and $68 \pm 5 \mathrm{mK}$ for the $\mathrm{x}, \mathrm{y}$ and $\mathrm{z}$ modes. The mean thermal occupation number $\langle n\rangle=k_{B} T_{j}^{f b} / \hbar \omega_{j}$ of the $\mathrm{y}$ mode is reduced from about $6.8 \times 10^{8}$ at $297 \mathrm{~K}$ to about 3400 at 1.5 mK. Fig. 6.21 shows the temperature of the three fundamental modes as a 
function of pressure. At low pressure and when the feedback gain is constant, the mode temperature should be proportional to the pressure, which is shown as a straight line with slope 1 in the figure. The temperature of the y mode agrees with this prediction very well at pressures above $1 \mathrm{~Pa}$.

At our lowest temperatures, the power spectra are still much larger than the noise level, and the minimum temperature is achieved at pressures

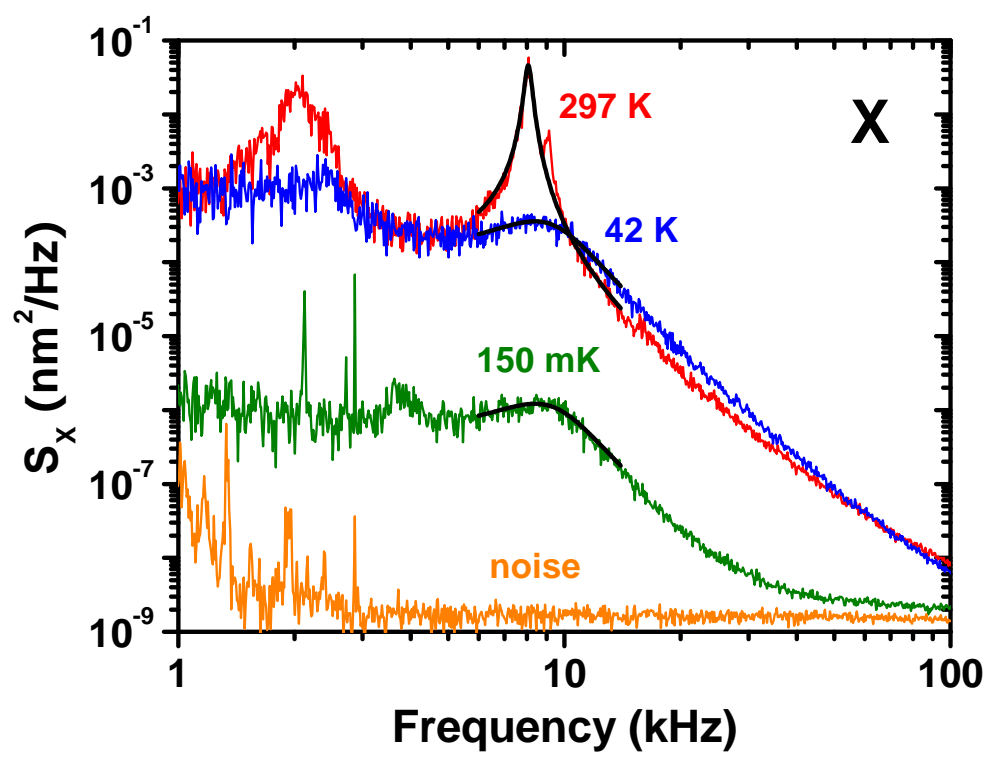

Figure 6.18: Power spectra of a trapped 3- $\mu \mathrm{m}$ diameter microsphere along the $\mathrm{X}$ axis as it is cooled. The red curve is the intrinsic spectrum at 637 $\mathrm{Pa}$ without feedback cooling, the blue curve is the spectrum at $637 \mathrm{~Pa}$ with feedback cooling, the green curve is the spectrum at $5.2 \mathrm{mPa}$ with feedback cooling, and the orange curve is the noise signal when there is no particle in the optical trap. The black curve is the fit of a thermal model (see text for details). We obtain mode temperatures from these fits. 
above the minimum pressure we can obtain, thus the electronic noise (in detection and feedback circuits) and the pressure are not the limiting factor of the current experiment. The dominant limiting factor is most likely residual coupling between the intensities and directions of the cooling beams. When we change the intensity of a cooling beam using an $\mathrm{AOM}$, the direction and profile of the beam is also changed slightly. This causes heating of the motion of a microsphere perpendicular to the beam while cooling it parallel. This problem should be solved by replacing the AOM's with electro-optic modulators (EOM's). The final temperature limited by the present detection system will be about $0.1 \mathrm{mK}$. Currently, the laser beam is attenuated before entering

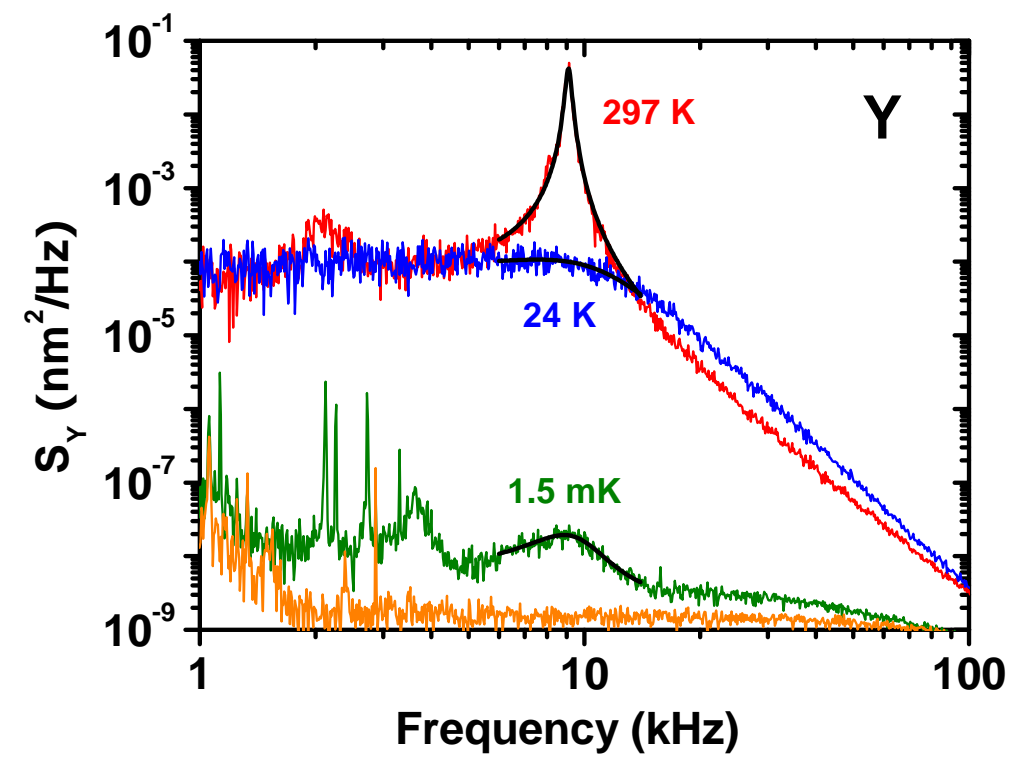

Figure 6.19: Power spectra of a trapped 3- $\mu \mathrm{m}$ diameter microsphere along the $\mathrm{Y}$ axis as it is cooled. The meanings of the curves are the same as in Fig. 6.18. 
the detectors because the laser power is larger than the damage threshold of the detectors. If we can utilize all of the signal contained in the laser beam for feedback cooling, the final temperature can be smaller than $0.01 \mathrm{mK}$, corresponding to a thermal occupation number in the order of 10 or less.

Our result is an important step toward quantum ground-state cooling of a trapped macroscopic object in vacuum by either cavity cooling[17-19] or feedback cooling with an improved detection and feedback scheme $[108,109]$. Our three-dimensional cooling enables future work on quantum superposition and entanglement of the motion between different directions. For cavity cool-

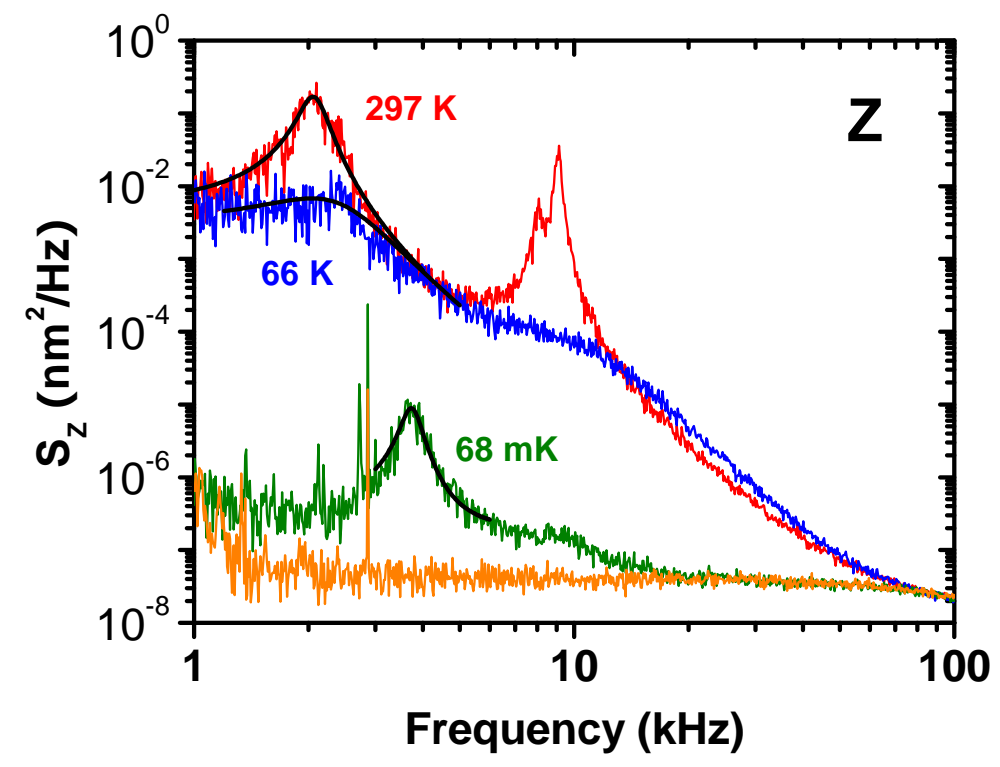

Figure 6.20: Power spectra of a trapped 3- $\mu \mathrm{m}$ diameter microsphere along the $\mathrm{Z}$ axis as it is cooled. The meanings of the curves are the same as in Fig. 6.18. 
ing of a trapped object in vacuum, it is also important to use feedback cooling to pre-cool and stabilize the object, in order to have enough time to tune the cavity cooling laser to the correct frequency for efficient cooling.

\subsection{Loss of microspheres in vacuum}

With feedback cooling, we have been able to trap a microsphere for more than one hour at pressure below $10^{-4}$ torr at optimal conditions. Ashkin et al. [45] had observed similar lifetimes when they levitated a $20-\mu$ m diameter sphere in vacuum (The laser intensity of the levitation trap is much smaller

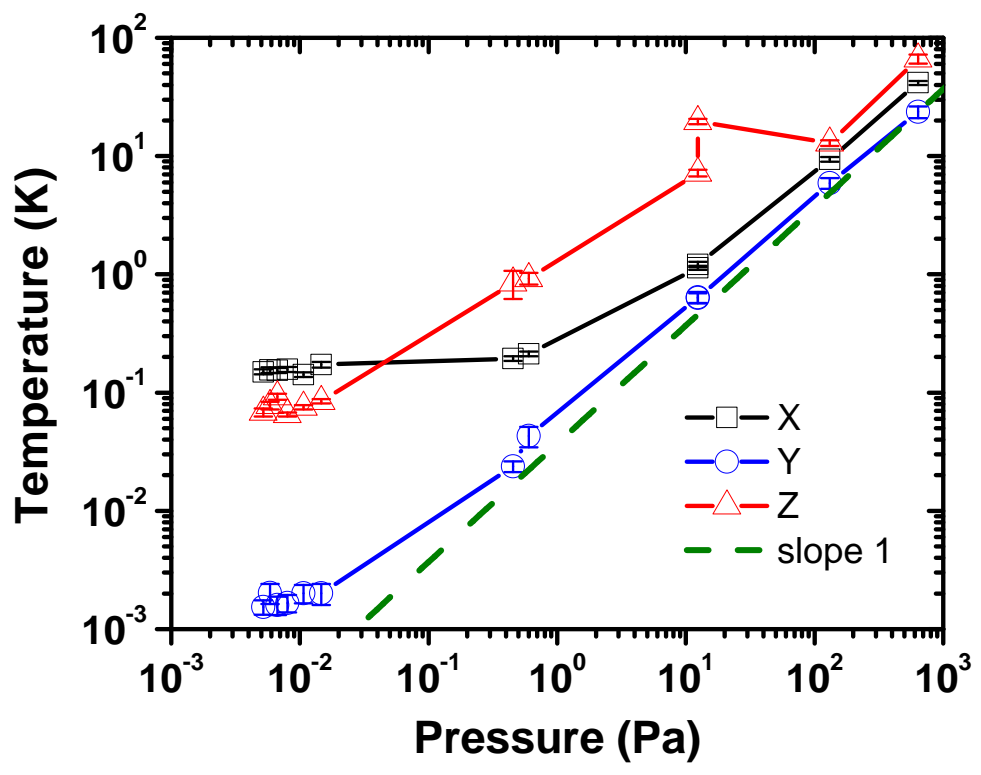

Figure 6.21: Temperatures of the three fundamental oscillation modes along $\mathrm{X}$ (black squares), Y (blue circles), and Z (red triangles) axes as a function of the air pressure. The dashed line is a straight line with slope 1 for comparison. 


\begin{tabular}{|l|l|l|l|l|}
\hline No. & $\begin{array}{l}\text { Total power } \\
\text { of trapping } \\
\text { beams }(\mathrm{mW})\end{array}$ & $\begin{array}{l}\text { Total power } \\
\text { of cooling } \\
\text { beams }^{+}(\mathrm{mW})\end{array}$ & $\begin{array}{l}\text { Lifetime with } \\
\text { ion pump on* } \\
\text { (minutes) }\end{array}$ & $\begin{array}{l}\text { Loss } \\
\text { pressure } \\
\text { (torr) }\end{array}$ \\
\hline \hline 1 & 160 & $\sim 60$ & 7 & $3.8 \times 10^{-5}$ \\
\hline 2 & 160 & $\sim 60$ & $21^{* *}$ & $2.4 \times 10^{-4}$ \\
\hline 3 & 130 & $\sim 60$ & 5 & $3.9 \times 10^{-5}$ \\
\hline 4 & 130 & $\sim 60$ & $11^{* *}$ & $2.6 \times 10^{-4}$ \\
\hline 5 & 130 & 3.6 & 26 & $9.4 \times 10^{-6}$ \\
\hline 6 & 130 & 6.5 & 22 & $7.7 \times 10^{-6}$ \\
\hline 7 & 113 & 39 & $\mathbf{8 8}$ & $2.4 \times 10^{-6}$ \\
\hline 8 & 82 & 19 & $\mathbf{6 9}$ & $2.5 \times 10^{-6}$ \\
\hline
\end{tabular}

Table 6.1: Examples of the lifetimes of a trapped 3.0- $\mu \mathrm{m}$ diameter microsphere in vacuum under different conditions. + The waists of the cooling beams are about $9 \mu \mathrm{m}$, so only parts of cooling beams pass through the trapped microsphere. ${ }^{*}$ The ion pump was turned on at about 1 mtorr; and the pressure dropped below $1.0 \times 10^{-4}$ torr within about 1 minute after the ion pump was on. ${ }^{* *}$ The ion pump was not turned on; the lifetime was the trapping time at pressures below 1 mtorr.

than the laser intensity of our dual-beam trap, and the size of the microsphere is much larger than ours). Table 6.1 shows some examples of measured lifetimes of a trapped microsphere in vacuum under different conditions. These lifetimes should be long enough to perform cavity cooling [17-19] and many other interesting experiments. Ideally, however, we would like the lifetime of the optical trap to be infinite even in vacuum.

Several things can affect the lifetime of a trapped microsphere in vacuum. For example, the alignment of the trapping beams and the gains of the feedback circuits can significantly affect the lifetime. We usually turn on feedback circuits at about 20 torr. At this pressure, the damping due to air is still 
large enough to keep the optical trap stable even if the feedback circuits are not tuned correctly. If the amplitude of the bead's motion increases when the feedback is turned on, the sign (polarity) of the velocity signal is incorrect, and must be inverted for the feedback loop to cool the motion. After tuning the feedback circuits correctly at 20 torr, we reduce the pressure slowly. The motion of the trapped microsphere usually becomes unstable when the pressure is reduced below 50 mtorr. We need to fine tune the horizontal and vertical directions of beam No. 2 with the two AOM's to restabilize the motion of the microsphere. After this step, we can reduce the pressure to below $10^{-5}$ torr. Because we need to fine tune the laser beams and the feedback circuits for each individual microsphere, the process is not very reproducible. Thus the lifetimes can be very different for different microspheres (Table 6.1).

We also found evidence that the ion pump intermittently undergoes electrical arcing [70] which kicks out a trapped microsphere. We observed spikes in the motion of trapped microspheres when we turned on the ion pump (an old ion pump that had not been used for several years). Sometimes the microsphere was lost immediately after we turned on the ion pump. We moved the ion pump further away from the optical trap to alleviate this problem. This problem disappeared after the ion pump was used for a few months. However, the lifetime of the optical trap in vacuum was still only on the order of 10 minutes. Then we stopped using the ion pump for a while, and used two sorption pumps in sequence to achieve lowest pressures of about $10^{-5}$ torr. However, we still observed the loss of microspheres after trapping for about 15 
minutes at low pressures. Then we considered the possibility that the sudden loss of trapped microspheres might be caused by floating dust in the air. A dust particle can cause fluctuations in the laser power and profile when it passes though the focus of a laser beam. So we used plastic sheets to seal the space between two lenses where a laser was focused in between. This method did not notably increase the lifetime of the trap in vacuum.

The final loss of a trapped microsphere is most likely caused by the heating due to light absorption. It seems that there is some dependance of the trap lifetime on the laser powers. The longest lifetime, 88 minutes, was observed when the total power of the trapping beams (1064nm) was $113 \mathrm{~mW}$ and the total power of the cooling beams (532nm) was $39 \mathrm{~mW}$. Because the waists of cooling beams were much larger than the size of the microsphere, only a part of cooling beams passed through the microsphere. However, silica has much larger absorption at $532 \mathrm{~nm}$ than $1064 \mathrm{~nm}$. So the heating effect of the cooling beams may be comparable to that of the trapping beams.

At pressures below 1 mtorr, the internal temperature of a trapped microsphere is mostly cooled by blackbody radiation $[17,45]$. The wavelength of a photon with energy of $k_{B} T$ is $48 \mu \mathrm{m}\left(\lambda=\frac{h c}{k_{B} T}\right.$, where $h$ is the Planck constant) at room temperature. This wavelength is much larger than the size of our microspheres. Thus we can treat the microsphere as a dipole in calculating the blackbody radiation when the internal temperature of the microsphere is not much higher than room temperature. If we assume that the microsphere has a constant and temperature-independent permittivity $\epsilon(\omega) \approx \epsilon_{b b}$ across 
the blackbody radiation spectrum, the microsphere radiates blackbody energy at a rate [17]:

$$
\frac{d E}{d t} \simeq-\frac{75}{\pi^{2}} \frac{V}{c^{3} \hbar^{4}} \operatorname{Im} \frac{\epsilon_{b b}-1}{\epsilon_{b b}+2}\left(k_{B} T_{\mathrm{int}}\right)^{5}
$$

where $V$ is the volume of the microsphere, and $T_{\text {int }}$ is the internal temperature of the microsphere. Similarly, the microsphere absorbs blackbody radiation from the environment at a rate:

$$
\frac{d E}{d t} \simeq \frac{75}{\pi^{2}} \frac{V}{c^{3} \hbar^{4}} \operatorname{Im} \frac{\epsilon_{b b}-1}{\epsilon_{b b}+2}\left(k_{B} T_{\text {env }}\right)^{5}
$$

where $T_{\text {env }}$ is the temperature of the environment.

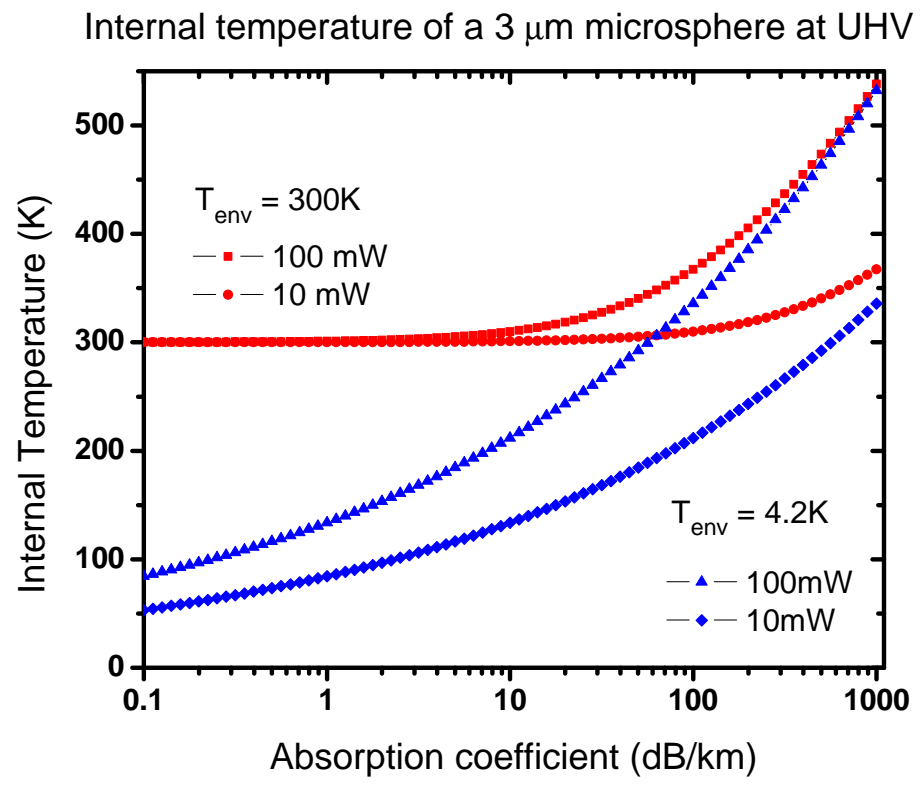

Figure 6.22: The equilibrium internal temperature of a trapped $3.0-\mu \mathrm{m}$ diameter microsphere irradiated by 10 and $100 \mathrm{~mW}$ of $1064 \mathrm{~nm}$ laser power in ultrahigh vacuum as a function of the absorption coefficient of the microsphere. 
In high vacuum, we can neglect the effect of background gas. The equilibrium internal temperature will be established when the sum of Eqs. 6.19 and 6.20 is equal to the heating rate due to light absorption of the laser beams. The absorbed energy of the microsphere from the laser beams is proportional to the power of lasers passing through the microsphere, and the optical absorption rate of the microsphere. Fig. 6.22 shows the calculated internal temperature of a trapped 3.0- $\mu \mathrm{m}$ diameter microsphere as a function of the environmental temperature, power of laser beams that pass through the microsphere, and the absorption rate of the microsphere.

When the environment is at room temperature and the power of the laser passing through the microsphere is $100 \mathrm{~mW}$, the internal temperature of the microsphere stays almost constant when the absorption coefficient is smaller than $10 \mathrm{~dB} / \mathrm{km}$ (Fig. 6.22). However, the internal temperature increases significantly if the absorption coefficient is $1000 \mathrm{~dB} / \mathrm{km}$ or higher. The microsphere will be lost when the internal temperature is above a certain threshold temperature. The silica microsphere is initially amorphous. It may undergo phase transition and become crystalline at high temperatures. Silica has many crystalline forms. The $\alpha$-quartz converts to $\beta$-quartz at $846 \mathrm{~K}$. At higher temperatures in vacuum, the silica microsphere will sublimate or melt and evaporate. The lifetime of the optical trap should be longer for a lower laser power.

Pure silica core optical fiber with loss of $0.148 \mathrm{~dB} / \mathrm{km}$ at $1570 \mathrm{~nm}$, $0.265 \mathrm{~dB} / \mathrm{km}$ at $1310 \mathrm{~nm}$, and $0.6 \mathrm{~dB} / \mathrm{km}$ at $1064 \mathrm{~nm}$ have been reported 
[116]. Whispering-gallery modes in fused-silica microspheres with quality factor of $Q=0.8 \times 10^{10}$ at $633 \mathrm{~nm}$ have been demonstrated experimentally [117]. This is close to the ultimate level determined by the fundamental material attenuation as measured in optical fibers $(0.7 \mathrm{~dB} / \mathrm{km}$ at $633 \mathrm{~nm})$. The loss increases significantly if the silica contains $\mathrm{OH}$ [118]. Commercial monodisperse silica microspheres are produced by the chemical reaction of tetraalkoxysilanes $\left(\mathrm{TEOS}, \mathrm{Si}\left(\mathrm{OC}_{2} \mathrm{H}_{5}\right)_{4}\right)$ in alcoholic solutions of water and ammonia $[119,120]$. Thus they are expected to contain $\mathrm{OH}, \mathrm{C}$, and $\mathrm{N}$ which will increase their absorption coefficient.

In the future, we should directly measure the internal temperature of an optically trapped microsphere using Raman spectroscopy[121]. This should give us a better insight of the final loss mechanism of a trapped microsphere in vacuum. We may also be able to purify a trapped microsphere in situ at high pressures by heating it with a $\mathrm{CO}_{2}$ laser [122]. Heating a trapped microsphere by a $\mathrm{CO}_{2}$ laser can melt the microsphere and reduce the size of the microsphere by evaporation. This also provides a novel method to produce and trap a nanosphere in air and vacuum. 


\section{Chapter 7}

\section{Towards quantum ground-state cooling}

Quantum ground-state cooling of an optically trapped microsphere in vacuum can be achieved by feedback cooling with an optimal detection and feedback scheme $[108,109]$. However, it will be difficult to achieve this since the real detection system and feedback circuits have electronic noise. Another method to achieve quantum-ground state cooling is cavity cooling $[7,9,96,123,124]$. Cavity cooling has been implemented in many different

micromechanical systems $[11,12,83,97-101,125,126]$, and has been used to successfully cool the mechanical vibration of a $100 \mathrm{~nm}$ thick aluminum membrane with a diameter of $15 \mu \mathrm{m}$ to the quantum ground state recently [14]. Recent theoretical calculations show that cavity cooling can be used to cool the mechanical motion of an optically trapped dielectric particle in vacuum to the quantum ground-state from room temperature [17-19, 102-106].

\subsection{Principle of cavity cooling}

As shown in Fig. 7.1(a), we consider a microsphere trapped in a cavity by a dual-beam optical tweezer. We use a dual-beam trap here because a single-beam trap requires a $\mathrm{NA} \approx 0.95$ objective lens. The working distance 
of a common high NA objective lens is too short (usually about $0.2 \mathrm{~mm}$ ) to integrate the optical cavity and the optical tweezer together. Thus we use two low NA lenses to create a dual-beam trap. In the system with which we have carried out feedback cooling, the distance between the two front surfaces of the two lenses is about $3.5 \mathrm{~mm}$. This should be enough for the cavity cooling laser to pass though.

Let the vibration frequency of the microsphere along the $z$ axis be $\omega_{M}$, the frequency of the cooling laser be $\omega_{L}$, the resonant frequency of the cavity be $\omega_{C}$, the intrinsic cavity linewidth be $\kappa$, and the rate of a photon scattered by the microsphere be $\gamma_{s c}$. For simplicity, we assume the linewidth of the cooling laser be much smaller than the cavity linewidth.
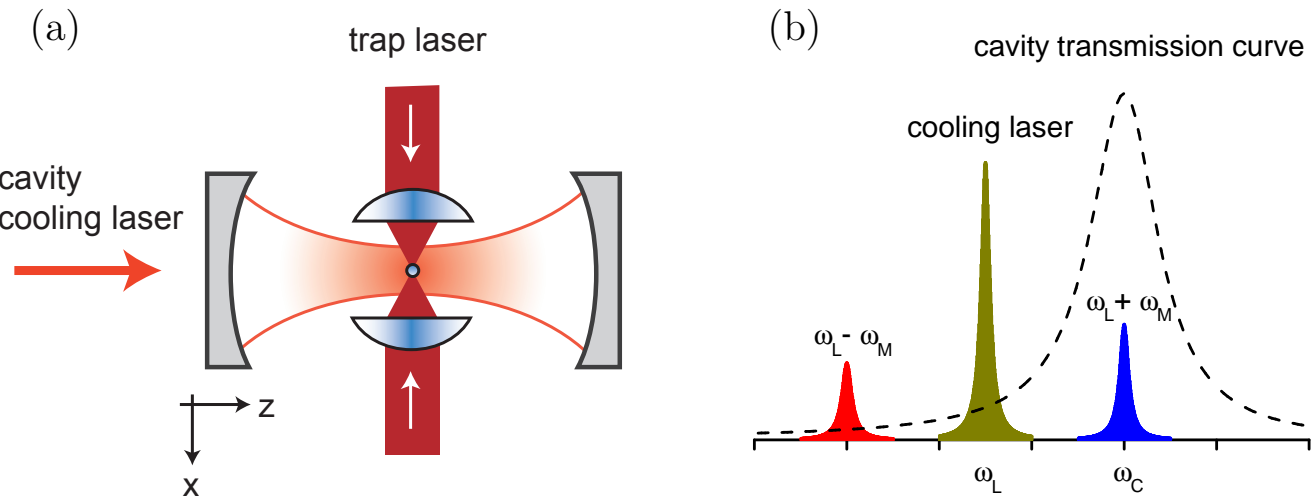

Figure 7.1: (a) Scheme of 1D cavity cooling. A microsphere is trapped inside an optical cavity with a dual-beam trap. (b) Principle of 1D cavity cooling. The frequency of the cooling laser $\left(\omega_{L}\right)$ is slightly smaller than the resonant frequency of the optical cavity $\left(\omega_{C}\right)$. The mechanical vibration of the trapped microsphere at frequency $\omega_{M}$ induces two side bands of the laser at frequencies of $\omega_{L}-\omega_{M}$ and $\omega_{L}+\omega_{M}$. 
When $\gamma_{s c} \ll \kappa \ll \omega_{M}$, the system is in the resolved-sideband limit. The mechanical vibration of the trapped microsphere will induce two side bands of the laser at frequencies of $\omega_{L}-\omega_{M}$ and $\omega_{L}+\omega_{M}$ (Fig. 7.1(b)). If the frequency of the cooling laser is red-detuned from than the resonant frequency of the cavity by $\omega_{M}$, the blue sideband $\omega_{L}+\omega_{M}$ will be on resonance with the cavity and the red sideband $\omega_{L}-\omega_{M}$ will be further away from the resonance of the cavity. Photons in the blue sideband leak out of the cavity because they are on resonance, while photons in the red sideband are trapped in the cavity. Thus on average the photons which come out of the cavity have larger frequency than the photons which come into the cavity. These photons carry away energy that is from the kinetic energy of the microsphere. Thus the photons cool the vibration of the trapped microsphere.

Let us consider a microsphere at position $z$ moving with momentum $p$ along the $z$ axis inside of a driven cavity. The microsphere causes the resonant frequency of a cavity with intrinsic resonant frequency $\omega_{0}^{C}$ to shift by an amount [17]:

$$
\delta \omega_{C}=-\frac{1}{2} \frac{\int d^{3} \mathbf{r} \delta P(\mathbf{r}) \cdot \mathbf{E}(\mathbf{r})}{\int d^{3} \mathbf{r} \epsilon_{0} \mathbf{E}^{2}(\mathbf{r})} \cdot \omega_{0}^{C},
$$

where $\mathbf{E}(\mathbf{r})$ is the bare cavity mode profile, and $\delta P(\mathbf{r})$ is the variation in permittivity introduced by the microsphere. Thus the resonant frequency of the cavity with microsphere is $\omega_{C}=\omega_{0}^{C}+\delta \omega_{C}$. If the diameter of the microsphere is much smaller than the wavelength of the laser, we can use Rayleigh approximation. In Rayleigh approximation, we have $P\left(\mathbf{r}^{\prime}\right) \approx \alpha_{\text {ind }} E(\mathbf{r}) \delta\left(\mathbf{r}-\mathbf{r}^{\prime}\right)$, where $\mathbf{r}$ is the center-of-mass position of the microsphere, $\alpha_{\mathrm{ind}}=3 \epsilon_{0} V\left(\frac{\epsilon-1}{\epsilon+2}\right)$ is 
its polarizability, $V$ is its volume, and $\epsilon$ is the electric permittivity.

The classical motion of the microsphere along the $z$ axis can be described by the following coupled equations for the cavity field $E$, the particle momentum and its position [127]:

$$
\begin{gathered}
\dot{E}=\left[-\kappa-\gamma_{s c}(z)+i \Delta_{C}-i \delta \omega_{C}(z)\right] E-\alpha, \\
\dot{p}=-|E|^{2} \frac{d}{d z} \delta \omega_{C}(z)-M \omega_{M}^{2} z \\
\dot{z}=p / M .
\end{gathered}
$$

Here $\Delta_{C}=\omega_{L}-\omega_{0}^{C}$ is the detuning of the laser relative to the resonant frequency of the empty cavity, and $\alpha$ describes the external pump laser. Because the cavity mode is a standing wave, the scattering rate $\gamma_{s c}(z)$ and the frequency shift $\delta \omega_{C}(z)$ have the shape of $\cos ^{2}(2 \pi z / \lambda)$. This classical model predicts the microsphere will stop as $t \rightarrow \infty$.

Quantum mechanical calculations show that the microsphere will not stop completely. In the limit $\omega_{M} \gg \kappa$ and taking the cooling rate $\Gamma^{\text {cool }} \approx \kappa$ (can be achieved by control the power and detuning of the cooling laser), the steady-state phonon number of the vibration is [17]:

$$
\left\langle n_{f}\right\rangle \approx \frac{\kappa^{2}}{16 \omega_{M}^{2}}+\frac{\gamma_{s c}}{\kappa} .
$$

Thus quantum ground-state cooling $\left(\left\langle n_{f}\right\rangle<1\right)$ can be achieved if $\omega_{M} \gg \kappa$ and $\kappa \gg \gamma_{s c}$. The value of $\gamma_{s c}$ depends on the size of the microsphere, the waist of the cavity mode and the length of the cavity. Calculations using Rayleigh approximation show that $\left\langle n_{f}\right\rangle<1$ can be achieved at reasonable conditions for 
microspheres with diameter smaller than $400 \mathrm{~nm}$ when the wavelength of the cooling laser is $1064 \mathrm{~nm}$ [106]. However, the Rayleigh approximation is only valid when the size of the microsphere is far smaller than the wavelength of the laser. It overestimates the scattering rate by about 3 orders of magnitude when the diameter of the microsphere is the same as the wavelength of the laser [56]. Thus we believe that ground-state cooling with a cavity should be possible for microspheres with diameter of $1 \mu \mathrm{m}$ or slightly larger.

\subsection{D ground-state cooling with a single cavity}

A microsphere will scatter the cooling laser to all three dimensions and cause 3D heating. The heating effects of laser noise are also 3D. If only onedimensional motion is cooled efficiently, the others will be heated continuously and the microsphere will eventually be kicked out of the trap. In order to achieve ground state cooling of an optically trapped nanosphere, we must use a 3D cooling scheme. We can add two more cavities for cooling the other two dimensions, but the system will become too complex to be realized experimentally. A better method to cool and measure the 3D motion of a nanosphere is to use the TEM00, TEM01, and TEM10 modes of a single cavity [19]. The TEM01 and TEM10 beams can be generated from a TEM00 beam by two phase plates [128]. Each one of these three modes can be coupled to the mo-

tion of a trapped microsphere along one orthogonal axis. Thus they can be used to cool and detect the 3D motion of a microsphere.

Trapping single atoms in a high-finesse cavity driven by three lasers at 
TEM00, TEM01, and TEM10 modes simultaneously has been demonstrated in an experiment already [129]. A recent experiment also used a tilted cavity TEM10 mode to measure the trajectories of single neutral atoms deterministically [130].

For simplicity, we consider a microsphere with size much smaller than the laser wavelength. In this case, the microsphere is in fact a nanosphere.

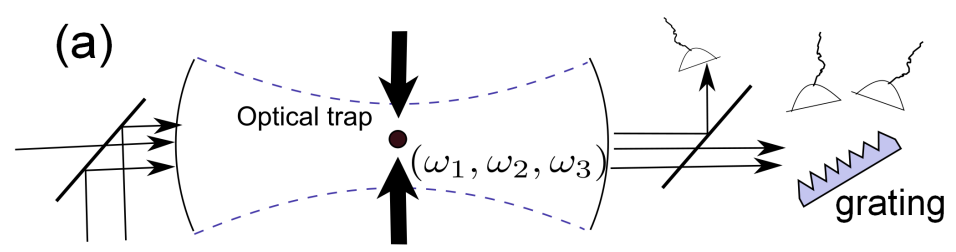

(b)

radial
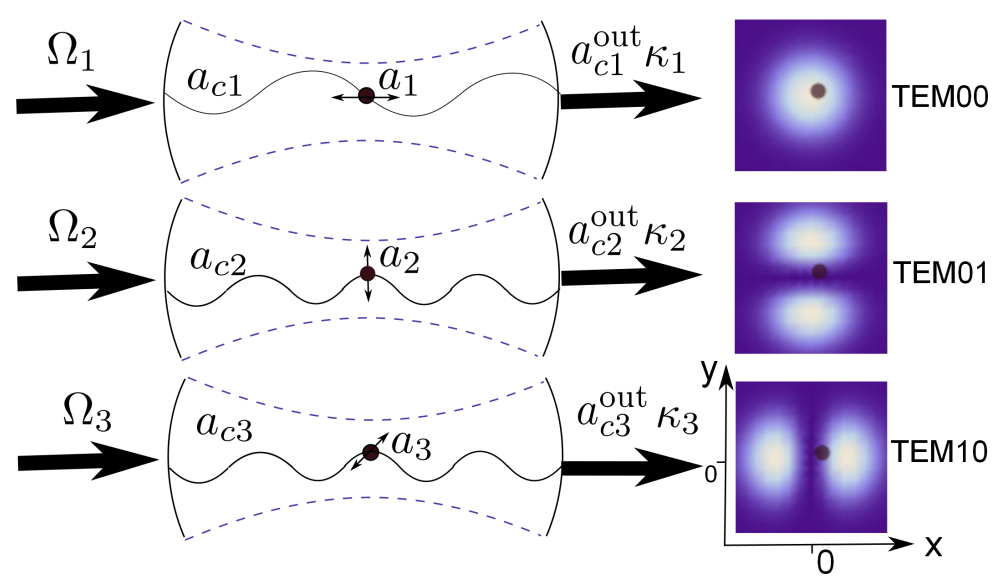

Figure 7.2: (a) A nanosphere is trapped by a dual-beam optical tweezer inside of a cavity. The cavity is driven by three lasers in TEM00, TEM01 and TEM10 modes to cool the 3D motion of the trapped nanosphere. (b) Three cooling modes TEM00, TEM01, and TEM10, and their radial distribution. The black dot represents the position of the trapped nanosphere. Figure is courtesy of Z. Q. Yin. 
The nanosphere is trapped in a cavity by an optical tweezer, as shown in Fig. 7.2. The trapping frequencies are $\omega_{1}, \omega_{2}$, and $\omega_{3}$ along the $z, x$, and $y$ axes, respectively. The cavity is driven by three lasers in TEM00, TEM01 and TEM10 modes to cool the 3D motion of the trapped nanosphere. The TEM01 laser is $s$-polarized, and the TEM00 and TEM10 lasers are p-polarized. The TEM01 and TEM10 lasers have almost the same frequency, and the TEM00 laser has a frequency different from those of the TEM01 and TEM10 lasers. Thus the TEM01 laser can be separated from the other two lasers by a polarizing beam splitter, and the TEM00 and TEM10 lasers can be separated by a grating. The frequency differences between the TEM00 and TEM01 lasers can be very large, and the TEM01 and TEM10 modes are orthogonal in polarizations. Therefore the interference between the three cavity modes can be neglected.

The TEM00 mode laser is used to cool the motion of the nanosphere along the $z$ axis, the TEM01 mode laser is for cooling the motion along the $y$ axis, and the TEM10 mode laser is for cooling the motion along the $x$ axis. The resonant frequencies of the cavity modes $a_{c 1}, a_{c 2}, a_{c 3}$ are $\omega_{c 1}, \omega_{c 2}$, $\omega_{c 3}$, respectively. The detunings between the lasers and the cavity modes are $\Delta_{c j}=\omega_{c}^{j}-\omega_{L}^{j}(j=1,2,3)$. Let us use $a_{j}$ to characterize the phonon mode along $q_{j}$ direction with $q_{1}=z, q_{2}=x, q_{3}=y, \Omega_{j}$ to be the driving strength of the lasers and $U_{j}$ to characterize the coupling between the cavity mode $a_{c j}$ 
and the nanosphere. In the limit that $\epsilon \gg 1$, we have [19]

$$
\begin{aligned}
U_{1} & =-\frac{3 V}{2 V_{c 1}} \exp \left(-\frac{2 x^{2}+2 y^{2}}{w^{2}}\right) \cos ^{2}\left(k_{1} z+\varphi_{1}\right) \omega_{c 1}, \\
U_{2} & =-\frac{3 V}{2 V_{c 2}} \frac{x^{2}}{w^{2}} \exp \left(-\frac{2 x^{2}+2 y^{2}}{w^{2}}\right) \cos ^{2}\left(k_{2} z+\varphi_{2}\right) \omega_{c 2}, \\
U_{3} & =-\frac{3 V}{2 V_{c 3}} \frac{y^{2}}{w^{2}} \exp \left(-\frac{2 x^{2}+2 y^{2}}{w^{2}}\right) \cos ^{2}\left(k_{3} z+\varphi_{3}\right) \omega_{c 3},
\end{aligned}
$$

where $V_{c 1}=(\pi / 4) L w^{2}$ and $V_{c 2}=V_{c 3}=(\pi / 16) L w^{2}$.

Let us assume the trapping lasers to be much stronger than cooling lasers, and neglect the effects of cooling lasers on trapping. If we carefully choose the location of the trap, such as $z_{0}=0, x_{0}=y_{0}=0.25 w, \varphi_{1}=\pi / 4$, and $\varphi_{2}=\varphi_{3}=0$, the gradients of the three light fields lie approximately along the three axes. The effective Hamiltonian is [19]

$$
\begin{aligned}
H_{e f f}= & \sum_{j=1}^{3}\left[\hbar \omega_{j} a_{j}^{\dagger} a_{j}-\hbar \Delta_{j} a_{c j}^{\dagger} a_{c j}+\frac{\hbar \Omega_{j}}{2}\left(a_{c j}+a_{c j}^{\dagger}\right)\right. \\
& \left.+\hbar g_{j} a_{c j}^{\dagger} a_{c j}\left(a_{j}+a_{j}^{\dagger}\right)\right],
\end{aligned}
$$

where $g_{j}=q_{j}^{z e r o} \partial U(x, y, z) /\left.\partial j\right|_{x=x_{0}, y=y_{0}, z=z_{0}}$ characterizes the coupling strength between the cavity mode and the oscillation of the nanosphere, and $q_{j}^{\text {zero }}=$ $\sqrt{\hbar / 2 M \omega_{j}}$ is the zero-point fluctuation for the phonon mode $a_{j}$. In general, $g_{1}$ can be one to two orders larger than $g_{2}$ and $g_{3}$. This effective Hamiltonian (7.6) is valid when the vibration amplitude of a trapped nanosphere is much smaller than the wavelength of the laser.

In the limit of resolved sideband cooling where $\omega_{j} \gg \kappa_{j}$, and when the driving strength is small, the final phonon number is $[19,131]$

$$
n_{m j}=-\frac{\left(\omega_{j}+\Delta_{c j}^{\prime}\right)^{2}+\left(\kappa_{j} / 2\right)^{2}}{4 \omega_{j} \Delta_{c j}^{\prime}}
$$


where $\Delta_{c j}^{\prime}$ is the effective detuning between the driving laser and the cavity mode $a_{c j}$. In the special case of $\Delta_{c j}^{\prime}=-\omega_{j}$, the final phonon number is $n_{m j}=\left(\kappa_{j} / 4 \omega_{j}\right)^{2} \ll 1$. Thus the motion of the microsphere can be cooled to ground state in all three dimensions in the resolved sideband regime. The cooling rate is $\Gamma_{j}=g_{j}^{2}\left|\alpha_{j}\right|^{2} /\left[\kappa_{j}\left(1+\frac{\kappa_{j}^{2}}{16 \omega_{j}^{2}}\right)\right]$, where $\alpha_{j}$ is the amplitude of the cavity mode $a_{c j}$.

\subsection{Heating effects of laser noise}

In a real experiment, the noise of lasers may cause significant heating of the motion of an optically trapped microsphere and may prevent groundstate cooling. The heating effect due to the photon shot noise of a laser has been found to be negligible [17]. However, the experiment is most likely to be limited by classical noise sources in the laser beams rather than the photon shot noise [19].

Let us first consider heating effects from the trapping laser [132]. In order to achieve ground-state cooling, the total heating rate from laser intensity fluctuation and laser pointing fluctuation should be much smaller than the cavity cooling rate. The relative intensity fluctuation of a laser is defined as $\epsilon(t)=\left(I(t)-I_{0}\right) / I_{0}$, where $I_{0}$ is the average laser intensity and $I(t)$ is the laser intensity at time $t$. The heating rate due to intensity fluctuation can be obtained by using a first-order time-dependent perturbation theory [132]:

$$
\Gamma_{\epsilon} \equiv \frac{\langle\dot{E}\rangle}{E}=\frac{\pi}{2} \omega_{j}^{2} S_{\epsilon}\left(2 \omega_{j}\right)
$$


where $S_{\epsilon}(\omega)=\frac{2}{\pi} \int_{0}^{\infty} d \tau \cos (\omega \tau)\langle\epsilon(t) \epsilon(t+\tau)\rangle$ is the one-sided power spectrum density of the relative intensity noise. For a trap frequency on the order of $\mathrm{MHz}, \Gamma_{\epsilon}$ approaches the order of $10^{-1} \mathrm{~Hz}$ when the $S_{\epsilon}(\omega)$ is on the order of $10^{-14} \mathrm{~Hz}^{-1}$.

The laser pointing fluctuation causes fluctuation of the position of the trap center. The heating rate due to pointing fluctuation is [132]:

$$
\Gamma_{j}^{p} \equiv \frac{\langle\dot{E}\rangle}{\hbar \omega_{j}}=\frac{\pi}{2} M \omega_{j}^{4} S_{j}\left(\omega_{j}\right) /\left(\hbar \omega_{j}\right),
$$

where $S_{j}(\omega)$ is the power spectrum density of position fluctuation of the trap center. Here the heating rate $\Gamma_{j}^{p}$ denotes the increase of the phonon number per second. If we want the $\Gamma_{j}^{p}$ to be on the order of $10^{-1} \mathrm{~Hz}$, we must have $S_{j}\left(\omega_{j}\right)$ to be about $10^{-35} \mathrm{~m}^{2} / \mathrm{Hz}$ for $\omega_{j} \sim 1 \mathrm{MHz}$. Experimentally, $S_{j}(\omega)$ has been able to be controlled to less than $10^{-34} \mathrm{~m}^{2} / \mathrm{Hz}$ for $\omega \sim \mathrm{kHz}[133]$. For frequencies much larger than the resonant frequencies of an instrument, $S_{j}\left(\omega_{j}\right)$ drops down quickly. Therefore, it should be possible to control the laser pointing fluctuation to be small enough to have $\Gamma_{j}^{p} \sim 0.1 \mathrm{~Hz}$.

For the cavity cooling laser, the dominate heating source is the phase noise of the laser [134-136]. The phase noise of the laser is related to the finite linewidth of the laser. The field of a laser with finite linewidth can be written as $\varepsilon(t)=\varepsilon e^{i \phi(t)}$, where the phase noise $\phi(t)$ is assumed to be Gaussian with zero mean value. For a Lorentzian noise spectrum with $S_{\dot{\phi}}(\omega)=2 \Gamma_{L} \gamma_{c} /\left(\gamma_{c}^{2}+\omega^{2}\right)$, and correlation function $\left\langle\dot{\phi(s)} \phi\left(\dot{s}^{\prime}\right)\right\rangle=\Gamma_{L} \gamma_{c} \exp \left(-\gamma_{c}\left|s-s^{\prime}\right|\right)$, where $\Gamma_{L}$ is the linewidth of the laser and $\gamma_{c}^{-1}$ is the correlation time of the phase noise, the 
minimum phonon number limited by the phase noise is [135]

$$
n_{p h}=n_{c} \frac{\Gamma_{L}}{\kappa} \frac{\gamma_{c}^{2}}{\gamma_{c}^{2}+\omega_{j}^{2}} .
$$

If $\Gamma_{L}=1 \mathrm{kHz}, \gamma_{c}=3 \mathrm{kHz}, \omega_{j}=10^{6} \mathrm{~Hz}$, and $n_{c}=10^{7}$, we have $n_{p h} \ll 1$. Thus the heating due to the phase noise of a cooling laser with linewidth on the order of $1 \mathrm{kHz}$ will not prevent ground-state cooling.

\subsection{Applications of cooled microspheres in vacuum}

\subsubsection{Measuring weak forces}

Optically trapped microspheres in water are sensitive to forces on the

order of $10^{-12} \mathrm{~N}$ [137], and have found extensive applications in biophysical research. For example, optical tweezers have been used to measure the force exerted by single kinesin molecules and the binding force between two individual stands of DNA molecules [138, 139].

An optically trapped microsphere in vacuum provides an even more sensitive force detector. The microsphere (just as a cantilever of an AFM) is subject to thermal fluctuation forces from the environment. The minimum force that a microsphere (or a cantilever of an AFM) can detect is linked to the friction that it experiences from the environment. The minimum detectable force is $[140]$ :

$$
F_{\text {min }}=\sqrt{4 M \Gamma_{0} k_{B} T \Delta_{b}}
$$

where $\Gamma_{0}$ is the viscous damping factor and $\Delta_{b}$ is the bandwidth of the measurement. From this equation, it is clear that a microsphere in vacuum is 
much more sensitive to a weak force than a microsphere in water because of the smaller damping $\Gamma_{0}$. A microsphere is also more sensitive to a weak force than a normal AFM cantilever because of its smaller mass $M$. Feedback cooling or cavity cooling do not affect the force sensitivity [141], but they can localize the position of the microsphere and benefit force measurements.

Figure 7.3 shows the calculated minimum detectable force by a $3-\mu \mathrm{m}$ microsphere at different pressures with $1 \mathrm{~Hz}$ bandwidth. The force sensitivity increases when the pressure decreases. $F_{\min }=2.9 \times 10^{-15} \mathrm{~N}$ at 1000 torr, $F_{\text {min }}=4.0 \times 10^{-18} \mathrm{~N}$ at $10^{-4}$ torr, and $F_{\min }=1.3 \times 10^{-21} \mathrm{~N}$ at $10^{-11}$ torr. The microsphere at 1000 torr is already much more sensitive than a common AFM cantilever, which can measure forces on the order of $1 \mathrm{nN}$.

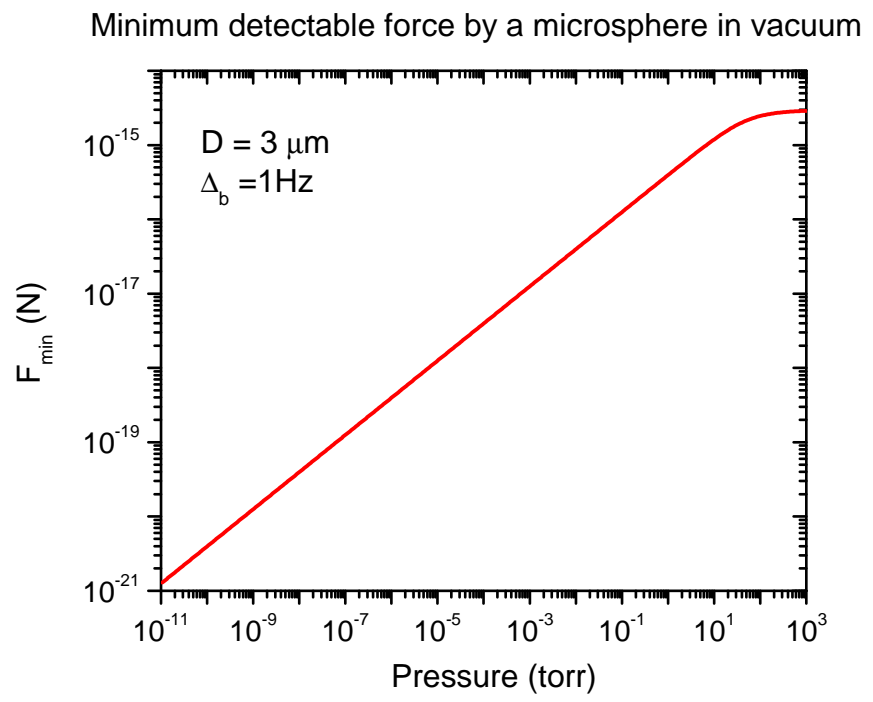

Figure 7.3: Minimum detectable force by a $3-\mu \mathrm{m}$ diameter microsphere trapped in vacuum. The measurement bandwidth is $1 \mathrm{~Hz}$. 
This potential force sensitivity is remarkable. For comparison, the Newtonian gravitational force between two point-like objects is

$$
F_{g}=\frac{G_{N} m_{1} m_{2}}{r^{2}}
$$

where $m_{1}$ and $m_{2}$ are two masses separated by distance $r$. The gravitational force between two $1 \mathrm{~g}$ masses separated by $1 \mathrm{~m}$ is $6.67 \times 10^{-17} \mathrm{~N}$.

It has been proposed that an optically trapped microsphere can be used to search for non-Newtonian gravity forces via an enhanced sensitivity of $10^{5}-10^{7}$ over current experiments at the $1 \mu \mathrm{m}$ length scale [142].

\subsubsection{Measuring the impact of single molecules}

The 3D cavity cooling scheme (Fig. 7.2) can also be used to measure the $3 \mathrm{D}$ motion of the nanosphere. In the limit of resolved sideband cooling where $\omega_{j} \gg \kappa_{j}$, and when the driving strength is small $\left(g_{j} \alpha_{j} \ll \kappa_{j}\right)$, the output

field from the cavity $a_{c j}^{\text {out }}$ is related to the phonon mode of the motion of the nanosphere $a_{j}$ by [19]

$$
a_{c j}^{\mathrm{out}}=-i \frac{2 g_{j} \alpha_{j}}{\sqrt{\kappa_{j}}} a_{j}+a_{c j}^{\mathrm{in}}
$$

Therefore the 3D motion of the nanosphere can be measured by detecting the output fields. In the resolved sideband limit, the output field is nearly vacuum, and will have a signal when there are collisions between the residual molecules in vacuum and the nanosphere.

If the collisions between the molecules and the nanosphere are elastic, and the nanosphere is in its ground state before the collisions, the average 
increase of the photon number for vibration mode $a_{j}$ after single collisions is $[19]$

$$
n_{j 0}=\frac{2 k_{B} T_{e n v} m_{m o l}}{\hbar \omega_{j} M},
$$

where $T_{e n v}$ is the temperature of the residual molecules that is the same as the environmental temperature, $m_{m o l}$ is the mass the molecule, and $M$ is the mass of the nanosphere. Since $n_{j 0}$ is proportional to $m_{m o l}$, we can distinguish the molecules with different masses in the measurement. The effective temperature $T_{j}$ of mode $a_{j}$ after single collisions can be obtained from

$$
\frac{1}{e^{\hbar \omega_{j} / k_{B} T_{j}}-1}=n_{j 0}
$$

The distribution of the average increase of the phonon number after single

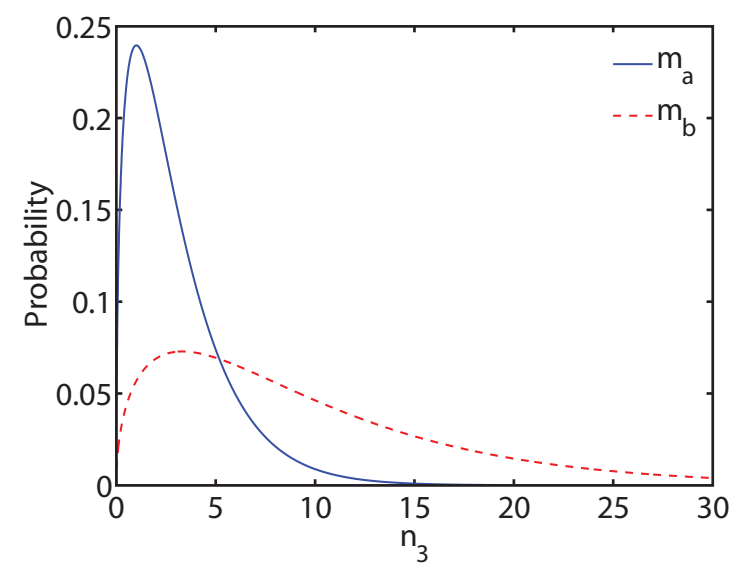

Figure 7.4: Distribution of the average increase of the phonon number of the mechanical mode $a_{3}$ after single elastic collisions between the nanosphere and molecules. The mass of the molecules is assumed to be $m_{a}=6.63 \times 10^{-26} \mathrm{~kg}$ (blue curve) or $m_{b}=2.18 \times 10^{-25} \mathrm{~kg}$ (red curve). The mass of the nanosphere is $m_{b}=1.03 \times 10^{-18} \mathrm{~kg}$. The temperature of the gas is $300 \mathrm{~K}$. Plot is courtesy of Z. Q. Yin. 
collisions is $[19]$

$$
f\left(n_{j}\right)=\frac{2}{\sqrt{\pi}}\left(\frac{\hbar \omega_{j}}{k_{B} T_{j}}\right)^{3 / 2} \exp \left(-n_{j} \frac{\hbar \omega_{j}}{k_{B} T_{j}}\right) .
$$

Fig. 7.4 shows the calculated distribution of mean phonon increase after single collisions with two different molecules. The two curves for the two different molecules are very different. Thus we can distinguish different molecules from the measurement. In the calculation, the radius of the nanosphere is $r=50 \mathrm{~nm}$ and the mass of the nanosphere is $M=1.03 \times 10^{-18} \mathrm{~kg}$

$\left(\rho=1.96 \mathrm{~g} / \mathrm{cm}^{3}\right)$. The optical tweezer is formed by a laser with power of $P_{t}=25 \mathrm{~mW}$ and wavelength of $\lambda=1500 \mathrm{~nm}$, focused by a lens with numerical aperture $N=0.9$. The trap frequency is $\left(\omega_{1}, \omega_{2}, \omega_{3}\right) / 2 \pi \simeq(0.5,0.5,0.2) \mathrm{MHz}$ [106]. The mass of the molecules is assumed to be $m_{a}=6.63 \times 10^{-26} \mathrm{~kg}$ (blue curve) or $m_{b}=2.18 \times 10^{-25} \mathrm{~kg}$ (red curve).

\subsubsection{Searching for gravity-induced quantum-state reduction}

A cooled microsphere in vacuum can also be used to study the gravityinduced quantum state reduction[2]. After cooling and creation of a superposition state in momentum, the optical trap can be switched off to let a microsphere undergo free-fall in vacuum [106]. The wavefuction will expand during free-fall and become a superposition state in space. The finite lifetime of a superposition due to gravity-induced state reduction is predicted to be on the order of $\hbar r /\left(G M^{2}\right)$ when the superposition is composed of states separated by a distance larger than the size of the microsphere $[2,6]$, where $G$ is Newton's gravitational constant. The predicted lifetime is about $3 \mathrm{~ms}$ for a $3-\mu \mathrm{m}$ 
diameter microsphere, which is shorter than the environmental decoherence time in good vacuum and thus measurable.

Other sources of decoherence must be minimized in order to measure the gravity-induced decoherence. The laser beams are switched off during the free fall of the microsphere. So the decoherence due to laser scattering can be neglected [16]. A dominate environmental decoherence source is the residual air molecules in the vacuum chamber. The localization rate due to air molecules is $\Lambda_{\text {air }}=8 \sqrt{2 \pi} m_{a} \bar{v} \operatorname{Pr}^{2} /\left(3 \sqrt{3} \hbar^{2}\right)$, where $P$ is the air pressure, $m_{a}$ is the mass of the air molecules and $\bar{v}$ is their thermal velocity [16]. $\Lambda_{\text {air }}$ can be reduced by reducing the air pressure and the environmental temperature.

There are many other applications of an optically trapped dielectric particle near the quantum ground state. Besides trapping glass microspheres, the dielectric particle can even be a virus or other living organism, in which case it would be possible to to produce Schrödinger cat states of living organisms [18]. 
Appendices 


\section{Appendix A}

\section{Physical properties of some common materials}

\begin{tabular}{|l|l|l|l|}
\hline Material & $\begin{array}{l}\text { Refractive } \\
\text { index } \\
(\text { at 589 nm })\end{array}$ & $\begin{array}{l}\text { Density } \\
\left(\mathrm{g} / \mathrm{cm}^{3}\right)\end{array}$ & $\begin{array}{l}\text { Dynamic } \\
\text { viscosity } \\
(\mu \mathrm{Pa} \cdot \mathrm{s})\end{array}$ \\
\hline \hline Vacuum & 1 & 0 & 0 \\
\hline Helium @ 0 ${ }^{\circ} \mathrm{C}, 1 \mathrm{~atm}$ & 1.000036 & 0.000176 & 18.69 \\
\hline Air @ 0 ${ }^{\circ} \mathrm{C}, 1 \mathrm{~atm}$ & 1.000292 & 0.00128 & 17.21 \\
\hline Air @ 27 ${ }^{\circ} \mathrm{C}, 1 \mathrm{~atm}$ & 1.000265 & 0.00116 & 18.54 \\
\hline \hline Liquid helium @ -269 ${ }^{\circ} \mathrm{C}$ & $1.0245^{a}$ & 0.129 & 3.319 \\
\hline Liquid nitrogen @ -196 ${ }^{\circ} \mathrm{C}$ & 1.199 & 0.8066 & 161.4 \\
\hline Water @ 20 ${ }^{\circ} \mathrm{C}$ & $1.3337^{b}$ & 0.9982 & 1002 \\
\hline Water @ 40 ${ }^{\circ} \mathrm{C}$ & 1.3310 & 0.9922 & 652.7 \\
\hline Water @ 60 ${ }^{\circ} \mathrm{C}$ & 1.3276 & 0.9832 & 466.0 \\
\hline Ethanol @ 20 ${ }^{\circ} \mathrm{C}$ & 1.361 & 0.7893 & $1074^{c}$ \\
\hline Acetone @ 20 ${ }^{\circ} \mathrm{C}$ & 1.359 & $0.7845^{c}$ & $306^{c}$ \\
\hline Ethylene glycol @ $20{ }^{\circ} \mathrm{C}$ & 1.432 & 1.114 & $16100^{c}$ \\
\hline \hline Ice (Ih) @ -7 ${ }^{\circ} \mathrm{C}$ & $1.31^{d}$ & $0.918^{d}$ & \\
\hline Fused silica & 1.458 & 2.21 & \\
\hline$\alpha$-Quartz & $1.544\left(\mathrm{n}_{o}\right), 1.553\left(\mathrm{n}_{e}\right)$ & 2.65 & \\
\hline Silica microspheres ${ }^{e}$ & $1.43-1.46$ & 2.0 & \\
\hline Polystyrene beads ${ }^{e}$ & 1.59 & 1.05 & \\
\hline Sapphire & $1.761\left(\mathrm{n}_{o}\right), 1.769\left(\mathrm{n}_{e}\right)$ & 3.97 & \\
\hline Diamond & 2.418 & 3.51 & \\
\hline
\end{tabular}

Table A.1: Physical properties of some common materials. The values are taken from reference [143]. ${ }^{a}$ at $546 \mathrm{~nm} ;{ }^{b}$ imaginary part of the refractive index of water and ice can be found at $[144,145] ;{ }^{c}$ at $25{ }^{\circ} \mathrm{C} ;{ }^{d}$ values from [146]; ${ }^{e}$ materials and values from Bangs Laboratories, Inc. [147]. 


\section{Bibliography}

[1] S. W. Hawking, and W. Israel (eds.). General Relativity; an Einstein Centenary Survey (Cambridge University Press, Cambridge, 1979).

[2] R. Penrose. On gravity's role in quantum state reduction. Gen. Rel. Grav., 28:581, 1996.

[3] L. Diósi. Models for universal reduction of macroscopic quantum fluctuations. Phys. Rev. A, 40:1165, 1989.

[4] G. C. Ghirardi, A. Rimini, and T. Weber. Unified dynamics for microscopic and macroscopic systems. Phys. Rev. D, 34:470, 1986.

[5] J. Christian. Testing gravity-driven collapse of the wave function via cosmogenic neutrinos. Phys. Rev. Lett., 95:160403, 2005.

[6] J. van Wezel, T. Oosterkamp, and J. Zaanen. Towards an experimental test of gravity-induced quantum state reduction. Phil. Mag., 88:1005, 2008.

[7] T. J. Kippenberg and K. J. Vahala. Cavity optomechanics: back-action at the mesoscale. Science, 321:1172, 2008.

[8] F. Marquadt and S. M. Girvin. Optomechanics. Physics, 2:40, 2009. 
[9] M. Aspelmeyer, S. Gröblacher, K. Hammerer, and N. Kiesel. Quantum optomechanics - throwing a glance. J. Opt. Soc. Am. B, 27:A189, 2010.

[10] B. Abbott et al. Observation of a kilogram-scale oscillator near its quantum ground state. New. J. Phys., 11:073032, 2009.

[11] Y. S. Park and H. Wang. Resolved-sideband and cryogenic cooling of an optomechanical resonator. Nature Phys., 5:489, 2009.

[12] S. Gröblacher et al. Demonstration of an ultracold micro-optomechanical oscillator in a cryogenic cavity. Nature Phys., 5:485, 2009.

[13] A. D. O'Connell et al. Quantum ground state and single-phonon control of a mechanical resonator. Nature, 464:697, 2010.

[14] J. D. Teufel et al. Sideband cooling micromechanical motion to the quantum ground state. http://arxiv.org/abs/1103.2144. (2011).

[15] T. Li, S. Kheifets, and M. G. Raizen. Millikelvin cooling of an optically trapped microsphere in vacuum. Nature Phys. doi: 10.1038/nphys1952 (2011).

[16] O. Romero-Isart, A. C. Pflanzer, F. Blaser, R. Kaltenbaek, N. Kiesel, M. Aspelmeyer, and J. I. Cirac. Large quantum superpositions and interference of massive nano-objects. http://arxiv.org/abs/1103.4081 (2011). 
[17] D. E. Chang et al. Cavity opto-mechanics using an optically levitated nanosphere. Proc. Natl. Acad. Sci. USA, 107:1005, 2010.

[18] O. Romero-Isart, M. L. Juan, R. Quidant, and J. Ignacio Cirac. Toward quantum superposition of living organisms. New J. Phys., 12:033015, 2010.

[19] Z.-Q. Yin, T. Li, and M. Feng. Three dimensional cooling and detection of a nanosphere with a single cavity. Phys. Rev. A, 83:013816, 2011.

[20] R. Brown. A brief account of microscopical observations made in the months of June, July and August, 1827, on the particles containded in the pollen of plants; and on the general existence of active molecules in organic and inorganic bodies. Phil. Mag., 4:161, 1828.

[21] F. M. Exner. Notiz zu Brown's molecularbewegung. Ann. d. Phys., $2: 843,1900$.

[22] M. Kerker. Brownian movement and molecular reality prior to 1900. J. Chem. Educ., 51:764, 1974.

[23] A. Einstein. Ann. d. Phys., 17:549, 1905.

[24] A. Einstein. Theoretische bemerkungen über die Brownsche bewegung. Zeit. f. Elektrochemie, 13:41, 1907.

[25] A. Einstein, Investigations on the theory of the Brownian movement, $\mathrm{R}$. Fürth, Ed., A. D. Cowper, Transl. (Methuen, London, 1926), pp. 63-67. 
[26] B. Lukić et al. Direct observation of nondiffusive motion of a Brownian particle. Phys. Rev. Lett., 95:160601, 2005.

[27] Y. Han et al. Brownian motion of an ellipsoid. Science, 314:626, 2006.

[28] I. Chavez, R. Huang, K. Henderson, E.-L. Florin, and M. G. Raizen. Development of a fast position-sensitive laser beam detector. Rev. Sci. Instrum., 79:105104, 2008.

[29] R. Huang, I. Chavez, K. M. Taute, B. Lukić, S. Jeney, M. G. Raizen, and E.-L. Florin. Direct observation of the full transition from ballistic to diffusive Brownian motion in a liquid. Nature Phys. doi:10.1038/nphys1953 (2011).

[30] P. D. Fedele and Y. W. Kim. Direct measurement of the velocity autocorrelation function for a Brownian test particle. Phys. Rev. Lett., 44:691, 1980.

[31] J. Blum et al. Measurement of the translational and rotational Brownian motion of individual particles in a rarefied gas. Phys. Rev. Lett., 97:230601, 2006.

[32] D. R. Burnham, P. J. Reece, and D. McGloin. Parameter exploration of optically trapped liquid aerosols. Phys. Rev. E, 82:051123, 2010.

[33] T. Li, S. Kheifets, D. Medellin, and M. G. Raizen. Measurement of the instantaneous velocity of a Brownian particle. Science, 328:1673, 2010. 
[34] R. Kubo. Brownian motion and nonequilibrium statistical mechanics. Science, 233:330, 1986.

[35] G. M. Wang, E. M. Sevick, E. Mittag, D. J. Searles, and D. J. Evans. Experimental demonstration of violations of the second law of thermodynamics for small systems and short time scales. Phys. Rev. Lett., 89:050601, 2002.

[36] R. Dean Astumian. Thermodynamics and kinetics of a Brownian motor. Science, 276:917, 1997.

[37] P. Hänggi and F. Marchesoni. Introduction: 100 years of Brownian motion. Chaos, 15:026101, 2005.

[38] J. C. Maxwell, A treatise on electricity and magnetism (1st ed.), 2:391 (Oxford, 1873).

[39] E. F. Nichols and G. F. Hull. The pressure due to radiation. The Astrophysical Journal, 17:315, 1903.

[40] P. N. Lebedev. Untersuchungen über die druckkräfte des lichtes. Ann. d. Phys., 6:433, 1901.

[41] E. F. Nichols and G. F. Hull. A preliminary communication on the pressure of light and heat radiation. Science, 14:588, 1901.

[42] A. Ashkin. History of optical trapping and manipulation of smallneutral particle, atoms, and molecules. IEEE Journal on selected topics in quantum electronics, 6:841, 2000. 
[43] A. Ashkin. Acceleration and trapping of particles by radiation pressure. Phys. Rev. Lett., 24:156, 1970.

[44] A. Ashkin and J. M. Dziedzic. Optical levitation by radiation pressure. Appl. Phys. Lett., 19:283, 1971.

[45] A. Ashkin and J. M. Dziedzic. Optical levitation in high vacuum. Appl. Phys. Lett., 28:333, 1976.

[46] A. Ashkin. Atomic beam deflection by resonance-radiation pressure. Phys. Rev. Lett., 25:1321, 1970.

[47] T. Hänsch and A. Schawlow. Cooling of gases by laser radiation. Opt. Commun., 13:68, 1975.

[48] A. Ashkin. Trapping of atoms by resonance radiation pressure. Phys. Rev. Lett., 40:729, 1978.

[49] D. J. Wineland, R. E. Drullinger, and F. L. Walls. Radiation-pressure cooling of bound resonant absorbers. Phys. Rev. Lett., 40:1639, 1978.

[50] A. Ashkin, J. M. Dziedzic, J. E. Bjorkholm, and S. Chu. Observation of a single beam gradient force optical trap for dielectric particles. Opt. Lett., 11:288, 1986.

[51] A. Ashkin and J. M. Dziedzic. Optical trapping and manipulation of viruses and bacteria. Science, 235:1517, 1987. 
[52] A. Ashkin, J. M. Dziedzic, and T. Yamane. Optical trapping and manipulation of single cells using infrared laser beams. Nature, 330:769, 1987.

[53] A. Ashkin. Forces of a single-beam gradient laser trap on a dielectric sphere in the ray optics regime. Biophys. J., 61:569, 1992.

[54] W. H. Wright, G. J. Sonek, and M. W. Berns. Parametric study of the forces on microspheres held by optical tweezers. Appl. Opt., 33:1735, 1994.

[55] R. Omori, T. Kobayashi, and A. Suzuki. Observation of a single-beam gradient-force optical trap for dielectric particle in air. Opt. Lett., $22: 816,1997$.

[56] Y. Harada and T. Asakura. Radiation forces on a dielectric sphere in the rayleigh scattering regime. Optics Comm., 124:529, 1996.

[57] P. C. D. Hobbs, Building electro-optical system: making it all work (2st ed.), 9 - 14 (Wiley, 2009).

[58] T. A. Nieminen, V. L. Y. Loke, A. B. Stilgoe, G. Kröner, A. M. Brańczyk, N. R. Heckenberg, and H. Rubinszteein-Dunlop. Optical tweezers computational toolbox. J. Opt. A: Pure Appl. Opt., 9:S196, 2007.

[59] K. C. Neuman and S. M. Block. Optical trapping. Rev. Sci. Instrum., $75: 2787,2004$. 
[60] B. V. Derjaguin, V. M. Muller, and Y. P. Toporov. Effect of contact deformations on the adhesion of particles. J. Colloid Interface Sci., 53:314, 1975 .

[61] L.-O. Heim, J. Blum, M. Preuss, and H.-J. Butt. Adhesion and friction forces between spherical micrometer-sized particles. Phys. Rev. Lett., 83:3328, 1999.

[62] M. Paajanen, J. Katainen, O. H. Pakarinen, A. S. Foster, and J. Lahtinen. Experimental humidity dependency of small particle adhesion on silica and titania. J. Colloid Interface Sci., 304:518, 2006.

[63] A. A. Busnaina, H. Lin, N. Moumen, J.-W. Feng, and J. Taylor. Particle adhesion and removal mechanisms in post-CMP cleaning processes. IEEE Transactions on semiconductor manufacturing, 15:374, 2002.

[64] S. W. Or and H. L. W. Chan. Mode coupling in lead zirconate titanate/epoxy 1-3 piezocomposite rings. J. Appl. Phys., 90:4122, 2001.

[65] A. Ashkin. Applications of laser radiation pressure. Science, 210:1081, 1980.

[66] A. van der Horst, High-refractive index particles in counter-propagating optical tweezers - manipulation and forces, $\mathrm{Ph}$. D thesis, Utrecht University, 2006. 
[67] Y. Roichman, B. Sun, A. Stolarski, and D. G. Grier. Influence of nonconservative optical forces on the dynamics of optically trapped colloidal spheres: the fountain of probability. Phys. Rev. Lett., 101:128301, 2008.

[68] P. Wu, R. Huang, C. Tischer, A. Jonas, and E.-L. Florin. Direct measurement of the nonconservative force field generated by optical tweezers. Phys. Rev. Lett., 103:108101, 2009.

[69] A. Pralle, M. Prummer, E.-L. Florin, E. H. K. Stelzer, and J. K. H. Hörber. Three-dimensional high-resolution particle tracking for optical tweezers by forward scattered light. Microscopy research and technique, 44:378, 1999 .

[70] D. M. Hoffman, B. Singh, J. H. Thomas III. Handbook of vacuum science and technology, page 237 (Academic Press, London, 1998).

[71] P. Langevin. C. R. Acad. Sci. (Paris), 146:530, 1908.

[72] D. S. Lemons and A. Gythiel. Paul Langevin's 1908 paper "on the theory of Brownian motion". Am. J. Phys., 65:1079, 1997.

[73] G. E. Uhlenbeck and L. S. Ornstein. On the theory of the Brownian motion. Phys. Rev., 36:823, 1930.

[74] M. C. Wang and G. E. Uhlenbeck. On the theory of the Brownian motion II. Rev. Mod. Phys., 17:323, 1945. 
[75] S. F. Nørrelykke, and H. Flyvbjerg. Harmonic oscillator in heat bath: exact simulation of time-lapse-recorded data, exact analytical benchmark statistics. arXiv:1102.0524, 2011.

[76] K. Berg-Sørensen and H. Flyvbjerg. Power spectrum analysis for optical tweezers. Rev. Sci. Instrum., 75:594, 2004.

[77] P. Kwee and B. Willke. Automatic laser beam characterization of monolithic Nd:YAG nonplanar ring lasers. Appl. Opt., 47:6022, 2008.

[78] K. G. Libbrecht and E. D. Black. Toward quantum-limited position measurements using optically levitated microspheres. Phys. Lett. A, 321:99, 2004.

[79] E. R. I. Abraham and E. A. Cornell. Teflon feedthrough for coupling optical fibers into ultrahigh vacuum systems. Appl. Opt., 37:1762, 1998.

[80] R. Zwanzig and M. Bixon. Compressibility effects in the hydrodynamic theory of Brownian motion. J. Fluid Mech., 69:21, 1975.

[81] A. Moshfegh, M. Shams, G. Ahmadi, and R. Ebrahimi. A novel surfaceslip correction for microparticles motion. Colloids and Surfaces A: Physicochem. and Eng. Aspects, 345:112, 2009.

[82] A. Hopkins, K. Jacobs, S. Habib, and K. Schwab. Feedback cooling of a nanomechanical resonator. Phys. Rev. B, 68:235328, 2003. 
[83] D. Kleckner and D. Bouwmeester. Sub-kelvin optical cooling of a micromechanical resonator. Nature, 444:75, 2006.

[84] N. L. Balazs. Brownian motion of a mirror in superfluid helium. Phys. Rev., 109:232, 1958.

[85] D. G. Henshaw and A. D. B. Woods. Modes of atomic motions in liquid helium by inelastic scattering of neutrons. Phys. Rev., 121:1266, 1961.

[86] I. N. Adamenko, K. E. Nemchenko, and I. V. Tanatarov. Transmission and reflection of phonons and rotons at the superfluid helium-solid interface. Phys. Rev. B, 77:174510, 2008.

[87] C. E. Brennen. A review of added mass and fluid inertial forces, Naval civil engineering laboratory, Report number: CR 82.010 (1982).

[88] E. J. Hinch. Application of the Langevin equantion to fluid susppensions. J. Fluid Mech., 72:499, 1975.

[89] D. Selmeczi et al. Brownian motion after Einstein and Smoluchowski: Some new applications and new experiments. Acta Physica Polonica B, $38: 2407,2007$.

[90] H. J. H. Clercx and P. P. J. M. Schram. Brownian particles in shear flow and harmonic potentials: A study of long-time tails. Phys. Rev. A, 46:1942, 1992. 
[91] Rongxin Huang, Brownian motion at fast time scales and thermal noise imaging, Ph. D dissertation, The University of Texas at Austin, 2008.

[92] B. Lukić et al. Motion of a colloidal particle in an optical trap. Phys. Rev. E, 76:011112, 2007.

[93] P. P. J. M. Schram and I. P. Yakimenko. On the theory of Brownian motion in compressible fluids. Physica A, 260:73, 1998.

[94] M. A. Taylor, J. Knittel, M. T. L Hsu, H-A Bachor, and W. P. Bowen. Sagnac interferometer-enhanced particle tracking in optical tweezers. $J$. Opt., 13:044014, 2011.

[95] W. Denk and W. W. Webb. Optical measurement of picometer displacements of transparent microscopic objects. Appl. Opt., 29:2382, 1990.

[96] P. F. Cohadon, A. Heidmann, and M. Pinard. Cooling of a mirror by radiation pressure. Phys. Rev. Lett., 83:3174, 1999.

[97] C. H. Metzger and K. Karrai. Cavity cooling of a microlever. Nature, 432:1002, 2004.

[98] A. Naik, O. Buu, M. D. LaHaye, A. D. Armour, A. A. Clerk, M. P. Blencowe, and K.C. Schwab. Cooling a nanomechanical resonator with quantum back-action. Nature, 443:193, 2006. 
[99] S. Gigan et al. Self-cooling of a micromirror by radiation pressure. Nature, 444:67, 2006.

[100] O. Arcizet, P.-F. Cohadon, T. Briant, M. Pinard, and A. Heidmann. Radiation-pressure cooling and optomechanical instability of a micromirror. Nature, 444:71, 2006.

[101] J. D. Thompson, B. M. Zwickl, A. M. Jayich, F. Marquardt, S. M. Girvin, and J. G. E. Harris. Strong dispersive coupling of a high-finesse cavity to a micromechanical membrane. Nature, 452:72, 2008.

[102] P. F. Barker and M. N. Shneider. Cavity cooling of an optically trapped nanoparticle. Phys. Rev. A, 81:023826, 2010.

[103] S. Singh, G. A. Phelps, D. S. Goldbaum, E. M. Wright, and P. Meystre. All-optical optomechanics: an optical spring mirror. Phys. Rev. Lett., 105:213602, 2010.

[104] R. J. Schulze, C. Genes, and H. Ritsch. Optomechanical approach to cooling of small polarizable particles in a strongly pumped ring cavity. Phys. Rev. A, 81:063820, 2010.

[105] P. F. Barker. Doppler cooling a microsphere. Phys. Rev. Lett., 105:073002, 2010.

[106] O. Romero-Isart, A. C. Pflanzer, M. L. Juan, R. Quidant, N. Kiesel, M. Aspelmeyer, and J. I. Cirac. Optically levitating dielectrics in the 
quantum regime: theory and protocools. Phys. Rev. A, 83:013803, 2011.

[107] A. Ashkin and J. M. Dziedzic. Feedback stabilization of optically levitated particles. Appl. Phys. Lett., 30:202, 1977.

[108] S. Mancini, D. Vitali, and P. Tombesi. Optomechanical cooling of a macroscopic oscillator by homodyne feedback. Phys. Rev. Lett., 80:688, 1998.

[109] C. Genes, D. Vitali, P. Tombesi, S. Gigan, and M. Aspelmeyer. Groundstate cooling of a micromechanical oscillator: comparing cold damping and cavity-assisted cooling schemes. Phys. Rev. A, 77:033804, 2008.

[110] S. A. Beresnev, V. G. Chernyak, and G. A. Fomyagin. Motion of a spherical particle in a rarefied gas. Part 2. Drag and thermal polarization. J. Fluid Mech., 219:405, 1990.

[111] L. Friedrich and A. Rohrbach. Improved interferometric tracking of trapped particles using two frequency-detuned beams. Opt. Lett., $35: 1920,2010$.

[112] T. P. Meyrath, Experiments with Bose-Einstein condensation in an optical box, Ph. D dissertation, The University of Texas at Austin, 2005.

[113] A. J. Trevitt, P. J. Wearne, and E. J. Bieske. Calibration of a quadrupole ion trap for particle mass spectrometry. International Journal of Mass Spectrometry, 262:241, 2007. 
[114] A. A. Sickafoose, J. E. Colwell, M. Horányi, and S. Robertson. Photoelectric changring of dust particles in vacuum. Phys. Rev. Lett., 84:6034, 2000.

[115] R. J. Clark, T. Lin, K. R. Brown, and I. L. Chuang. A two-dimensional lattice ion trap for quantum simulation. J. Appl. Phys., 105:013114, 2009 .

[116] K. Nagayama et al. Ultra low loss $(0.1484 \mathrm{~dB} / \mathrm{km})$ pure silica core fiber. Sei Technical Review, 57:3, Jan. 2004.

[117] M. L. Gorodetsky, A. A. Savchenkov, and V. S. Ilchenko. Ultimate Q of optical microsphere resonators. Opt. Lett., 21:453, 1996.

[118] B. J. Skutnik, B. Foley, and K. B. Moran, High numerical aperture silica core fibers. Progress in biomedical optics and imaging, SPIE, 2004.

[119] A. van Blaaderen, J. van Geest, and A. Vrij. Monodisperse colloidal silica spheres from tetraalkoxysilanes: particle formation and growth mechanism. J. Col. Inter. Sci., 154:481, 1992.

[120] G. De, B. Karmakar, and D. Ganguli. Hydrolysis-condensation reactions of TEOS in the presence of acetic acid leading to the generation of glasslike silica microspheres in solution at room temperature. J. Mater. Chem., 10:2289-2293, 2000. 
[121] J.F. Lübben, C. Mund, B. Schrader, and R. Zellner. Uncertainties in temperature measurements of optically levitated single aerosol particles by Raman spectroscopy. J. Mol. Structure, 480-481:311-316, 1999.

[122] A. D. McLachlan and F. P. Meyer. Temperature dependence of the extinction coefficient of fused silica for $\mathrm{CO}_{2}$ laser wavelengths. Appl. Opt., 26:1728, 1987.

[123] I. Wilson-Rae, N. Nooshi, W. Zwerger, and T. J. Kippenberg. Theory of ground state cooling of a mechanical oscillator using dynamical backaction. Phys. Rev. Lett., 99:093901, 2007.

[124] F. Marquardt, J. P. Chen, A. A. Clerk, and S. M. Girvin. Quantum theory of cavity-assisted sideband cooling of mechanical motion. Phys. Rev. Lett., 99:093902, 2007.

[125] A. Schliesser, O. Arcizet, R. Rivière, G. Anetsberger, and T. J. Kippenberg. Resolved-sideband cooling and position measurement of a micromechanical oscillator close to the Heisenberg uncertainty limit. $\mathrm{Na}$ ture Phys., 5:509, 2009.

[126] T. Rocheleau et al. Preparation and detection of a mechanical resonator near the ground state of motion. Nature, 463:72, 2010.

[127] P. Horak, G. Hechenblaikner, K. M. Gheri, H. Stecher, and H. Ritsch. Cavity-induced atom cooling in the strong coupling regime. Phys. Rev. Lett., 79:4974, 1997. 
[128] T. P. Meyrath, F. Schreck, J. L. Hanssen, C.-S. Chuu, and M.G. Raizen. A high frequency optical trap for atoms using Hermite-Gaussian beams. Opt. Express, 13:2843, 2005.

[129] T. Puppe, I. Schuster, A. Grothe, A. Kubanek, K. Murr, P. W. H. Pinkse, and G. Rempe. Trapping and observing single atoms in a blue-detuned intracavity dipole trap. Phys. Rev. Lett., 99:013002, 2007.

[130] P. Zhang, Y. Guo, Z. Li, Y. Zhang, Y. Zhang, J. Du, G. Li, J. Wang, and T. Zhang. Elimination of the degenerate trajectory of a single atom strongly coupled to a tilted $\mathrm{TEM}_{10}$ cavity mode. Phys. Rev. A, 83:031804(R), 2011.

[131] A. A. Clerk, M. H. Devoret, S. M. Girvin, F. Marquardt, and R. J. Schoelkopf. Introduction to quantum noise, measurement and amplification. Rev. Mod. Phys., 82:1155, 2010.

[132] T. A. Savard, K. M. O'Hara, and J. E. Thomas. Laser-noise-induced heating in far-off resonance optical traps. Phys. Rev. A, 56:R1095, 1997.

[133] T. Corbitt, C. Wipf, T. Bodiya, D. Ottaway, D. Sigg, N. Smith, S. Whitcomb, and N. Mavalvala. Optical dilution and feedback cooling of a gram-scale oscillator to $6.9 \mathrm{mK}$. Phys. Rev. Lett., 99:160801, 2007.

[134] Lajos Diósi. Laser linewidth hazard in optomechanical cooling. Phys. Rev. A, 78:021801(R), 2008. 
[135] P. Rabl, C. Genes, K. Hammerer, and M. Aspelmeyer. Phase-noise induced limitations on cooling and coherent evolution in optomechanical systems. Phys. Rev. A, 80:063819, 2009.

[136] Zhang-qi Yin. Phase noise and laser-cooling limits of optomechanical oscillators. Phys. Rev. A, 80:033821, 2009.

[137] R. M. Simmons, J. T. Finer, S. Chu, and J. A. Spudich. Quantitative measurements of force and displacement using an optical trap. Biophys. J., 70:1813, 1996.

[138] S. C. Kuo and M. P. Sheetz. Force of single kinesin molecules measured with optical tweezers. Science, 260:232, 1993.

[139] U. Bockelmann, Ph. Thomen, B. Essevaz-Roulet, V. Viasnoff, and F. Heslot. Unzipping DNA with optical tweezers: High sequence sensitivity and force flips. Biophys. J., 82:1537, 2002.

[140] S. Kuehn, S. A. Hickman, and J. A. Marohn. Advances in mechanical detection of magnetic resonance. J. Chem. Phys., 128:052208, 2008.

[141] J. L. Garbini, K. J. Bruland, W. M. Dougherty, and J. A. Sidles. Optimal control of force microscope cantilevers. I. controller design. J. Appl. Phys., 80:1951, 1996.

[142] A. A. Geraci, S. B. Papp, and J. Kitching. Short-range force detection using optically cooled levitated microspheres. Phys. Rev. Lett., 105:101101, 2010. 
[143] W. M. Haynes (ed.). CRC Handbook of Chemistry and Physics, 91st edition. CRC-Press, 2010.

[144] L. Kou, D. Labrie, and P. Chylek. Refractive indices of water and ice in the 0.65- to 2.5- $\mu$ m spectral range. Appl. Opt., 32:3531, 1993.

[145] S. G. Warren and R. E. Brandt. Optical constants of ice from the ultraviolet to the microwave: A revised compilation. J. Geophys. Res., 113:D14220, 2008.

[146] S. G. Warren. Optical constants of ice from ultraviolet to the microwave. Appl. Opt., 23:1206, 1984.

[147] Bangs Labratories, Inc. http://www.bangslabs.com/. 


\section{Vita}

Tongcang Li was born in Wenzhou, Zhejiang Province, China in 1982. He completed his high school education from Cangnan No. 1 Middle School (later renamed Cangnan Middle School Zhejiang Province) in 2000. He received the Bachelor of Science degree in Physics from the University of Science and Technology of China in 2004. He entered the graduate school at the University of Texas at Austin in 2004 and joined Mark Raizen's group in November 2004.

Permanent address: 267 Jinshan Road Apt. 501

Qianku Town, Cangnan County

Wenzhou, Zhejiang 325804, China

This dissertation was typeset with $\mathrm{HT}_{\mathrm{E}} \mathrm{X}^{\dagger}$ by the author.

\footnotetext{
${ }^{\dagger} \mathrm{HT}_{\mathrm{E}} \mathrm{X}$ is a document preparation system developed by Leslie Lamport as a special version of Donald Knuth's TEX Program.
} 